Prepared for the U.S. Department of Energy

under Contract DE-AC05-76RL01830

\title{
Survey of Revegetated Areas on the Fitzner/Eberhardt Arid Lands Ecology Reserve: Status and Initial Monitoring Results
}
JL Downs
RE Durham
SO Link
$\mathrm{R}$ Cruz
L Rozeboom
$S$ McKee

September 2011

Pacific Northwest

NATIONAL LABORATORY

Proudly Operated by Battelle Since 1965 


\title{
DISCLAIMER
}

This report was prepared as an account of work sponsored by an agency of the United States Government. Neither the United States Government nor any agency thereof, nor Battelle Memorial Institute, nor any of their employees, makes any warranty, express or implied, or assumes any legal liability or responsibility for the accuracy, completeness, or usefulness of any information, apparatus, product, or process disclosed, or represents that its use would not infringe privately owned rights. Reference herein to any specific commercial product, process, or service by trade name, trademark, manufacturer, or otherwise does not necessarily constitute or imply its endorsement, recommendation, or favoring by the United States Government or any agency thereof, or Battelle Memorial Institute. The views and opinions of authors expressed herein do not necessarily state or reflect those of the United States Government or any agency thereof.

\author{
PACIFIC NORTHWEST NATIONAL LABORATORY \\ operated by \\ BATTELLE \\ for the \\ UNITED STATES DEPARTMENT OF ENERGY \\ under Contract DE-AC05-76RL01830
}

Printed in the United States of America
Available to DOE and DOE contractors from the Office of Scientific and Technical Information,
P.O. Box 62, Oak Ridge, TN 37831-0062;
ph: (865) 576-8401
fax: $(865) 576-5728$
email: reports@adonis.osti.gov

Available to the public from the National Technical Information Service

5301 Shawnee Rd., Alexandria, VA 22312

ph: (800) 553-NTIS (6847)

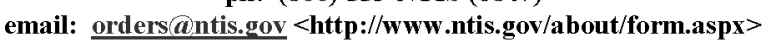

Online ordering: http://www.ntis.gov

This document was printed on recycled paper. 


\section{Survey of Revegetated Areas on the Fitzner/Eberhardt Arid Lands Ecology Reserve: Status and Initial Monitoring Results}

$\begin{array}{ll}\text { JL Downs } & \text { RE Durham } \\ \text { SO Link } & 1 \\ \text { L Rozeboom } & \text { R Cruz } \\ & \text { S McKee }\end{array}$

September 2011

Prepared for

CH2M HILL Plateau Remediation Company under Contract DE-AC05-76RL01830

Pacific Northwest National Laboratory

Richland, Washington 99352

\footnotetext{
${ }^{1}$ Department of Science and Engineering, Confederated Tribes of the Umatilla Indian Reservation, Pendleton, Oregon.
} 


\section{Summary}

During 2010, the U.S. Department of Energy (DOE), Richland Operations Office removed a number of facilities and debris from the Fitzner/Eberhardt Arid Lands Ecology Reserve (ALE), which is part of the Hanford Reach National Monument (HRNM). Revegetation of disturbed sites is necessary to stabilize the soil, reduce invasion by exotic weeds, and to accelerate re-establishment of native plant communities. Seven revegetation units were identified on ALE based on soils and potential native plant communities. Native seed mixes and plant material were identified for each unit based on the desired plant community. Revegetation of locations affected by decommissioning of buildings and debris removal was undertaken during the winter and early spring of 2010 and 2011, respectively. This report describes both the details of planting and seeding for each of the units, describes the sampling design for monitoring, and summarizes the data collected during the first year of monitoring.

In general, the revegetation efforts were successful in establishing native bunchgrasses and shrubs on most of the sites within the 7 revegetation units. Invasion of the revegetation areas by exotic annual species was minimal for most sites, but was above initial criteria in 3 areas: the Hodges Well subunit of Unit 2, and Units 6 and 7. 


\section{Acronyms and Abbreviations}

ALE

$\mathrm{CCCF}$

CTUIR

DOE

HQ

HRNM

PNNL
Fitzner/Eberhardt Arid Lands Ecology Reserve

Combined Community Communications Facility

Confederated Tribes of the Umatilla Indian Reservation

U.S. Department of Energy

ALE Ecology Headquarters

Hanford Reach National Monument

Pacific Northwest National Laboratory 


\section{Contents}

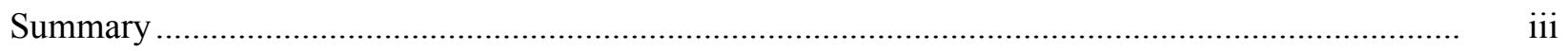

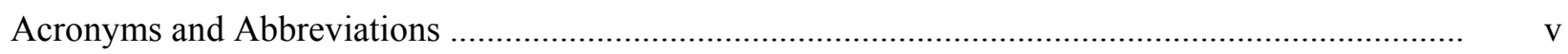

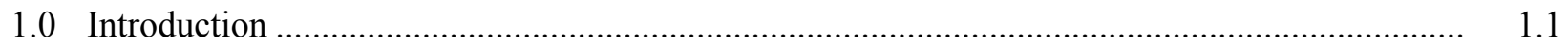

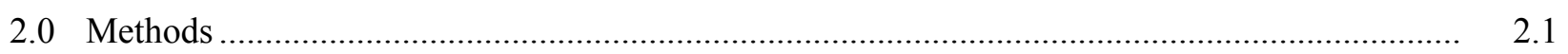

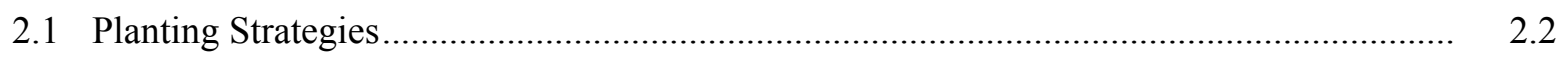

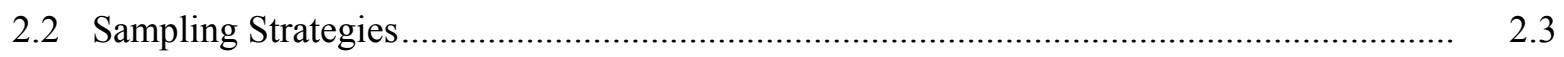

2.2.1 Modified Whittaker Sampling Strategy ........................................................... 2.3

2.2.2 Monitoring Strategy for CTUIR Diversity Plantings ........................................ 2.4

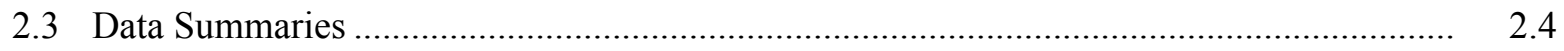

3.0 Revegetation Status and Initial Monitoring Results ......................................................... 3.1

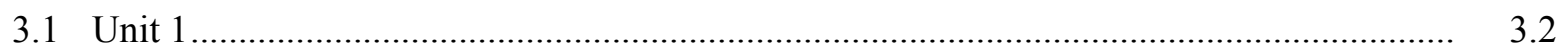

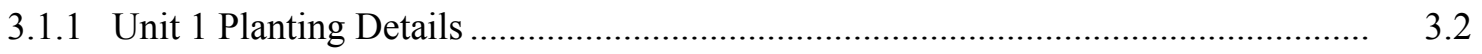

3.1.2 Unit 1 Vegetation Establishment................................................................. 3.3

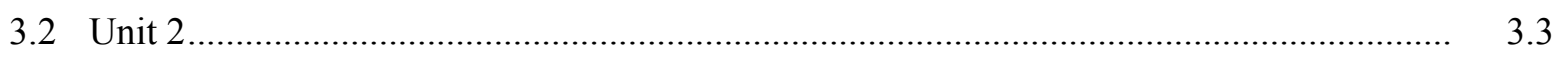

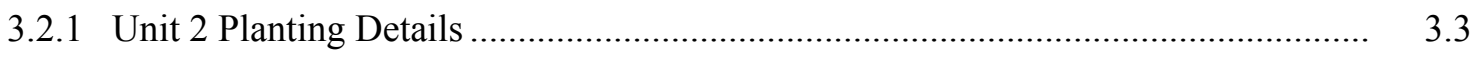

3.2.2 Unit 2 Vegetation Establishment................................................................ 3.6

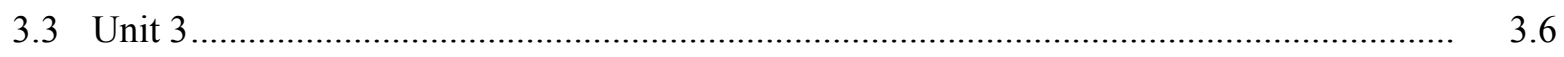

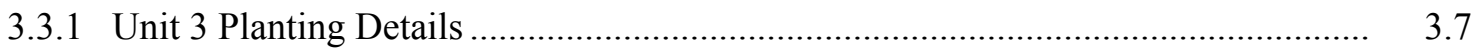

3.3.2 Unit 3 Vegetation Establishment.................................................................. 3.8

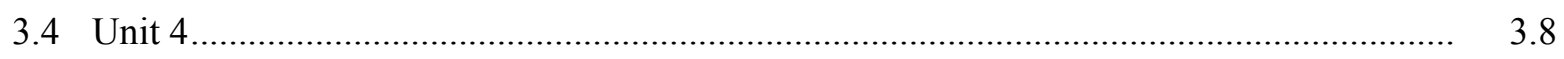

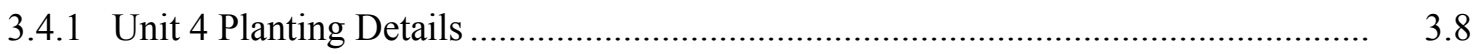

3.4.2 Unit 4 Vegetation Establishment.................................................................. 3.11

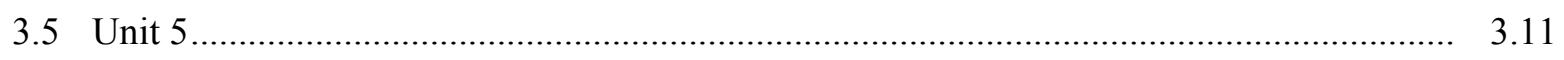

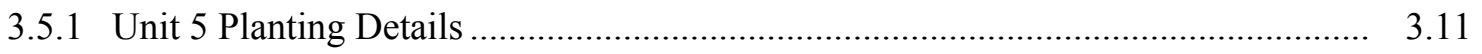

3.5.2 Unit 5 Vegetation Establishment.................................................................. 3.12

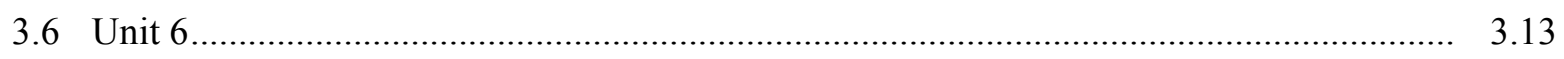

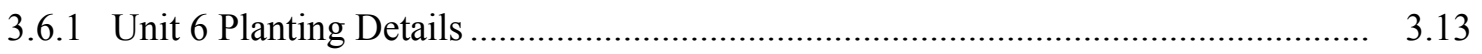

3.6.2 Unit 6 Vegetation Establishment.................................................................. 3.13

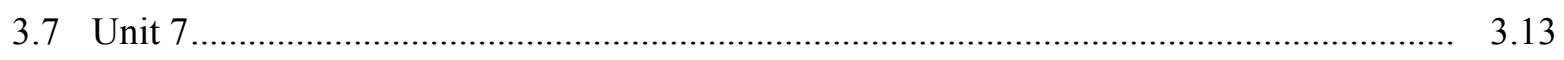

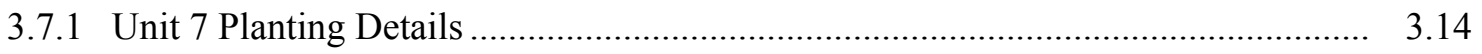

3.7.2 Unit 7 Vegetation Establishment................................................................. 3.15

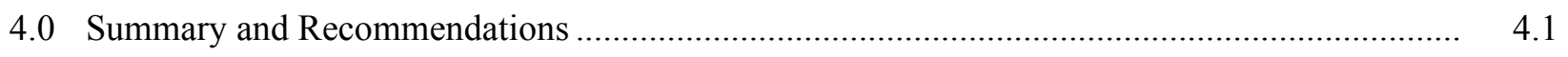

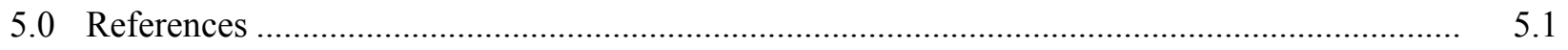

Appendix A - Planting Details ....................................................................................... A.1

Appendix B - Plot Locations and Photographs .................................................................... B.1 
Appendix C - Data Collected on Revegetation Monitoring Plots on ALE During FY 2011 _............ C. C.1

Appendix D - Restoration After Construction Disturbance on the Arid Lands Ecology Reserve ...... D.1

\section{Figures}

1.1 Relative Locations of Revegetation Units on the Arid Lands Ecology Reserve....................... 1.2

2.1 Diagram of Modified Whittaker Sample Plot Design .............................................................. 2.4

\section{Tables}

2.1 Revegetation Sites and Units

3.1 Summary of Native Species Density and Richness and Exotic Species Cover Measured in 2011 on ALE Revegetation Areas and on Two Nearby

Reference Areas

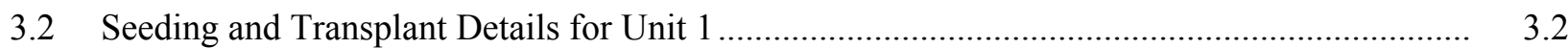

3.3 Seeding and Transplant Detail for the ALE Ecology and Hodges Well Subunits in Unit 2...... 3.4

3.4 Seeding and Transplant Detail for Debris Sites 50, 60, 109, 138, and 146......................... 3.5

3.5 Seeding and Transplanting Detail for Debris Sites 40, 127, and 139 ................................... 3.6

3.6 Initial Monitoring Results for Vegetation Characteristics Measured on Subunits

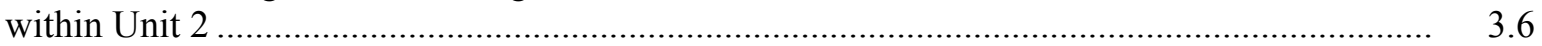

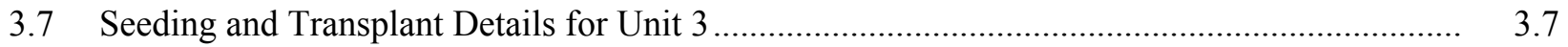

3.8 Seeding Detail for Unit 4 (Subunit 623A) ..................................................................... 3.8

3.9 Seeding and Transplant Details for Unit 4 (Subunit 6652-C) ................................................. 3.9

3.10 Seeding and Transplant Detail for Unit 4 (Subunit CCCF) ................................................ 3.10

3.11 Seeding and Transplant Details for Unit 5 .............................................................. 3.12

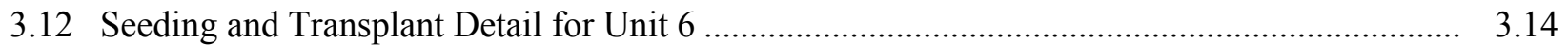

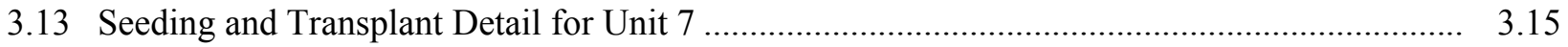




\subsection{Introduction}

During 2010, the U.S. Department of Energy (DOE), Richland Operations Office removed a number of facilities and debris from the Fitzner/Eberhardt Arid Lands Ecology Reserve (ALE), which is part of the Hanford Reach National Monument (HRNM). Revegetation of locations affected by decommissioning of buildings and debris removal was specified by the Environmental Assessment (DOE 2009; DOE/EA-1660F) and by Stipulation C.7 of the Memorandum of Agreement (MOA) for the Rattlesnake Mountain Combined Community Communication Facility and Infrastructure Cleanup on the Fitzner/Eberhardt Arid Lands Ecology Reserve, Hanford Site, Richland Washington (DOE 2009). Revegetation of disturbed sites is necessary to stabilize the soil, reduce invasion of these areas by exotic weeds, and to accelerate re-establishment of native plant communities.

The excavation of buildings and debris removal resulted in varying levels and extents of soil and surface disturbance at a number of locations on ALE shown in Figure 1.1. Revegetation strategies and guidance for implementing revegetation actions were developed for groups of locations (revegetation units) based on level of disturbance, elevation, soil type, and potential native vegetation (Downs et al. 2011). Revegetation actions were implemented on approximately 10 acres between November 2010 and March 2011 by Pacific Northwest National Laboratory (PNNL) and their subcontractors in cooperation with the Confederated Tribes of the Umatilla Indian Reserve (CTUIR) under funding from CH2M HILL Plateau Remediation Company. PNNL and CTUIR also cooperated in initial monitoring surveys of the revegetation units in June and July 2011. This report provides detailed descriptions of the revegetation actions implemented for individual locations and units, describes the sampling methods used to conduct initial monitoring surveys in 2011, and summarizes the monitoring results to assess native plant establishment on these disturbed sites. 


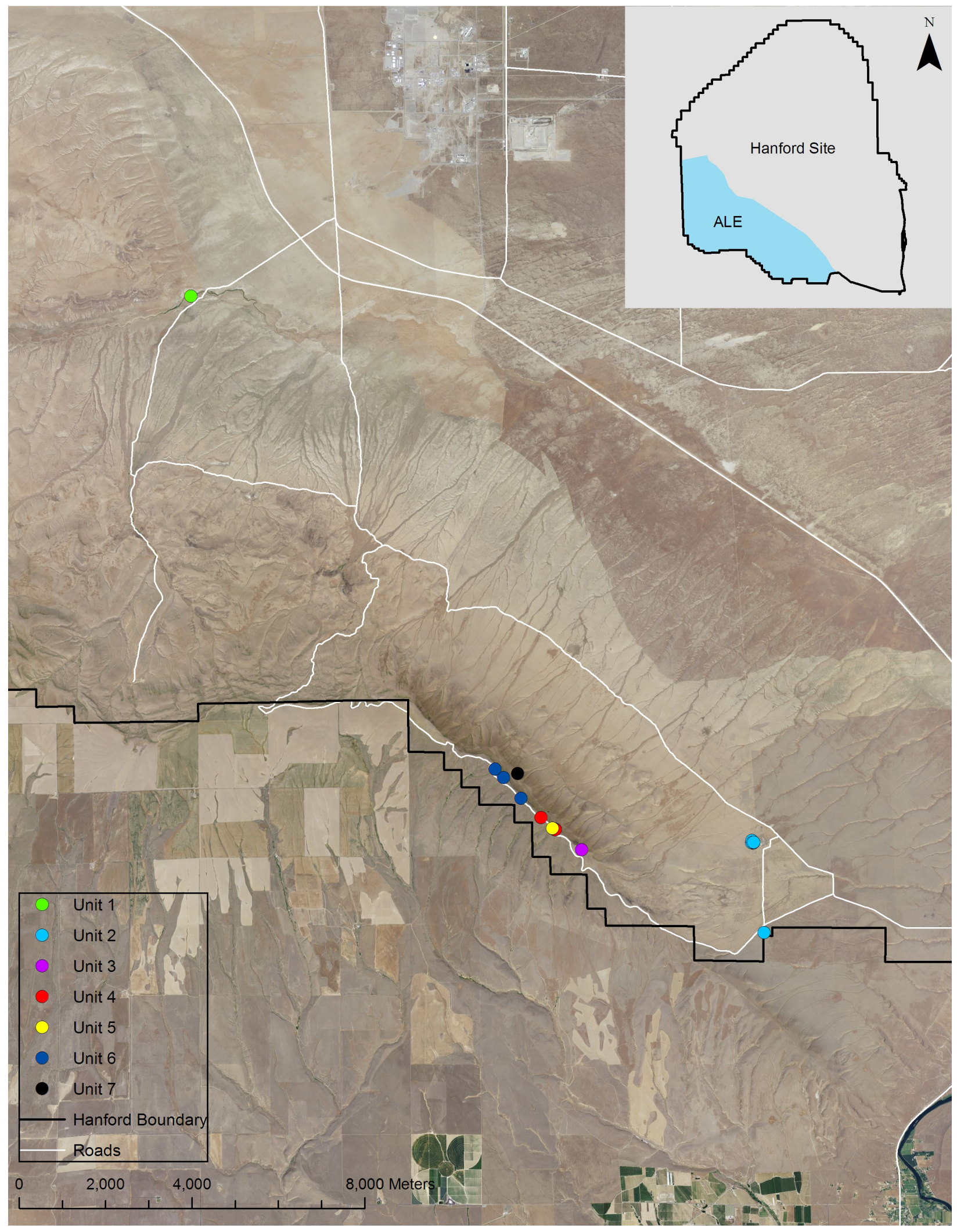

Figure 1.1. Relative Locations of Revegetation Units on the Arid Lands Ecology Reserve 


\subsection{Methods}

This study was conducted on the ALE Reserve, which lies on the northern slope of Rattlesnake Ridge near Richland, Washington. The semi-arid ALE Reserve is characterized as shrub-steppe habitat, with elevations ranging from 600 to 3600 feet above mean sea level. The average annual precipitation is $16 \mathrm{~cm}$, occurring mostly in the autumn and winter (Stone et al. 1983). Seven revegetation units were identified in the revegetation plan (Downs et al. 2011) based on soils, plant community, and elevation (Table 2.1). The individual sites were prepared for revegetation, and then seeded and planted with the appropriate mix of native species between November 2010 and March 2011.

Table 2.1. Revegetation Sites and Units (from Downs et al. 2011)

\begin{tabular}{|c|c|c|c|c|}
\hline Site & Location & Habitat Type & $\begin{array}{l}\text { Revegetation } \\
\text { Unit }\end{array}$ & $\begin{array}{l}\text { Estimated } \\
\text { Size } \\
\text { (acres) }\end{array}$ \\
\hline $\begin{array}{l}646 \text { - Aquatic } \\
\text { Research Laboratory }\end{array}$ & $\begin{array}{l}\text { East of } \\
\text { Rattlesnake } \\
\text { Springs }\end{array}$ & $\begin{array}{l}\text { Black greasewood (Sarcobatus } \\
\text { vermiculatus)/Saltgrass (Distichilis } \\
\text { stricta) }\end{array}$ & Unit 1 & 0.5 \\
\hline $\begin{array}{l}6652-\mathrm{G}, \mathrm{H}, \mathrm{I}, \mathrm{J}, \mathrm{M} \text {, and } \\
\text { parking areas; }\end{array}$ & $\begin{array}{l}\text { ALE Ecology } \\
\text { Buildings, } \\
1200 \mathrm{ft} \text { elevation }\end{array}$ & $\begin{array}{l}\text { Big sagebrush (Artemisia tridentata)/ } \\
\text { Bluebunch wheatgrass } \\
\text { (Pseudoroegnaria spicata) }\end{array}$ & Unit 2 & 2.0 \\
\hline $\begin{array}{l}8 \text { miscellaneous debris } \\
\text { sites }\end{array}$ & $\begin{array}{l}8 \text { sites; } 1000 \text { to } \\
1600 \mathrm{ft} \text { elevation }\end{array}$ & Big sagebrush/Bluebunch wheatgrass & Unit 2 & 1.7 \\
\hline $\begin{array}{l}\text { Hodges Well Pump } \\
\text { House and Roadway } \\
(6630)\end{array}$ & $\begin{array}{l}\sim 1 \text { mi south of } \\
\text { ALE Buildings, } \\
1200 \mathrm{ft} \text { elevation }\end{array}$ & Big sagebrush/Bluebunch wheatgrass & Unit 2 & 0.52 \\
\hline $\begin{array}{l}\text { Radio telescope site } \\
\text { (6631) and road }\end{array}$ & $\begin{array}{l}\text { Approximately } \\
3000 \mathrm{ft} \text { elevation } \\
\text { alongside } \\
\text { 106-road to ridge } \\
\text { top }\end{array}$ & $\begin{array}{l}\text { Big sagebrush/bluebunch wheatgrass- } \\
\text { Idaho fescue (Festuca idahoensis) and } \\
\text { Thyme buckwheat (Eriogonum } \\
\text { thymoides)/bluebunch wheatgrass }\end{array}$ & $\begin{array}{c}\text { Unit } 3- \\
\text { Transitional }\end{array}$ & 0.36 \\
\hline $\begin{array}{l}6652-C \text { Building and } \\
\text { parking area, including } \\
6652-D \text { and } 6632\end{array}$ & Top of ridge & $\begin{array}{l}\text { Thyme buckwheat/Bluebunch } \\
\text { wheatgrass and Big sagebrush/ } \\
\text { Bluebunch wheatgrass-Idaho fescue }\end{array}$ & Unit 4 & 1.3 \\
\hline $623 \mathrm{~A}$ & Top of ridge & $\begin{array}{l}\text { Thyme buckwheat/Bluebunch } \\
\text { wheatgrass and Big sagebrush/ } \\
\text { Bluebunch wheatgrass-Idaho fescue }\end{array}$ & Unit 4 & 0.35 \\
\hline $\begin{array}{l}\text { 6652-C Shed, } \\
\text { Telescope Dome, and } \\
\text { Berm Areas }\end{array}$ & Top of ridge & $\begin{array}{l}\text { Thyme buckwheat /Bluebunch } \\
\text { wheatgrass and Eastside Canyon } \\
\text { Shrublands }\end{array}$ & Unit 5 & 1.2 \\
\hline $\begin{array}{l}\text { Powerline Roadway- } \\
\text { New underground } \\
\text { power service }\end{array}$ & $\begin{array}{l}\text { Just below } \\
6652-C \text { Building } \\
\text { area Rattlesnake } \\
\text { Ridge }\end{array}$ & $\begin{array}{l}\text { Thyme buckwheat/Bluebunch } \\
\text { wheatgrass and Big sagebrush/ } \\
\text { Bluebunch wheatgrass-Idaho fescue }\end{array}$ & Unit 4 & 0.2 \\
\hline $\begin{array}{l}\text { Combined Community } \\
\text { Communications } \\
\text { Facility }\end{array}$ & $\begin{array}{l}\text { Along } \\
\text { Rattlesnake } \\
\text { Ridge }\end{array}$ & $\begin{array}{l}\text { Thyme buckwheat/Bluebunch } \\
\text { wheatgrass and Big sagebrush/ } \\
\text { Bluebunch wheatgrass-Idaho fescue }\end{array}$ & Unit 4 & 0.34 \\
\hline
\end{tabular}


Table 2.1. (contd)

\begin{tabular}{|c|c|c|c|c|}
\hline Site & Location & Habitat Type & $\begin{array}{l}\text { Revegetation } \\
\text { Unit }\end{array}$ & $\begin{array}{l}\text { Estimated } \\
\text { Size } \\
\text { (acres) }\end{array}$ \\
\hline Crown Castle (6635) & $\begin{array}{l}\text { Along } \\
\text { Rattlesnake } \\
\text { Ridge }\end{array}$ & $\begin{array}{l}\text { Thyme buckwheat/Bluebunch } \\
\text { wheatgrass and Big sagebrush/ } \\
\text { Bluebunch wheatgrass-Idaho fescue }\end{array}$ & Unit 6 & 0.1 \\
\hline $\begin{array}{l}6636 \text { and } 6637 \\
\text { Communication } \\
\text { Towers and Access } \\
\text { Roads }\end{array}$ & $\begin{array}{l}\text { Along } \\
\text { Rattlesnake } \\
\text { Ridge }\end{array}$ & $\begin{array}{l}\text { Thyme buckwheat/Bluebunch } \\
\text { wheatgrass and Big sagebrush/ } \\
\text { Bluebunch wheatgrass-Idaho fescue }\end{array}$ & Unit 6 & 0.6 \\
\hline 6652T-Pumphouse & Below Ridgeline & Eastside Canyon Shrublands-Riparian & Unit 7 & 0.89 \\
\hline
\end{tabular}

\subsection{Planting Strategies}

Because each revegetation site and unit differ in terms of soils, elevation, potential native vegetation, extent of disturbance, and accessibility, a suite of methods were used to seed and plant native species. Seed mixes for each site were specified based on the dominant grasses and shrubs of the potential native plant community, and the availability of forb species appropriate for that community. Site preparation and seeding methods for each planting area were determined based on the following considerations:

- amount of soil and surface disturbance and the condition of the substrate

- whether native plant species persisted within the disturbance footprint

- the topography and access to the site.

Drill seeding, broadcast seeding and other hand-seeding methods were employed depending on the site, substrate condition, and access for equipment.

In addition, islands of both seeded and transplanted native forbs and shrubs were installed by the CTUIR to create the potential for increased native diversity within the disturbance footprints. The CTUIR provided technical services by: collecting and processing native seed stocks for diversity islands; propagating transplant materials; installing the diversity islands; and field monitoring and reporting of vegetation establishment on the island plots. Transplanted islands in Unit 1 were irregular in shape $\left(4-\mathrm{m}^{2}\right)$, and shrubs were also planted along the roadway. Unit 2 islands were circular with a radius of $5 \mathrm{~m}$. Island plots in Units 3 through 7 were circular with a radius of $4 \mathrm{~m}$ except where circumstances required planting in other irregular patterns. Seeded island plots were circlular with varying radii. Plots at low elevation (Units 1 and 2) had radii ranging from 2.6 to $1.96 \mathrm{~m}$ depending on the number of seeds applied. Plots at higher elevation (Units 3 through 6) had radii ranging from 1.39 to $0.544 \mathrm{~m}$. No seeded islands were installed on Unit 7. Each of the island plots installed by the CTUIR was marked at the center with a spike or rebar with a numbered aluminum tag that was wired into the ground.

The specific methods used, planting details, seed mixes applied and the number and types (seeded or transplanted) of diversity islands at each site are described in Section 3. Seeding rates and transplant densities are provided in Appendix A. 


\subsection{Sampling Strategies}

Two types of sampling were conducted to evaluate the revegetation units. A modified Whittaker plot design was used to sample the general vegetation characteristics on the revegetation units. Plots that were seeded or planted by the CTUIR to create islands of diversity were sampled separately. Sampling of all areas was conducted in June and July 2011. Units were sampled in approximate order of increasing elevation, starting with Unit 1, so that the lower elevation, drier and warmer locations were sampled earlier in the growing season before vegetation senesced.

\subsubsection{Modified Whittaker Sampling Strategy}

At each location, a modified Whittaker approach (Stohlgren et al. 1995) was used to survey the establishment of vegetation on each of the units by species. The modified Whittaker plot design uses a series of nested plots to sample plant species at different scales. The modified Whittaker approach utilizes 3 plots of different sizes:

- a $1000-\mathrm{m}^{2}$ plot $(20 \mathrm{~m} \times 50 \mathrm{~m})$, where only species presence is recorded

- a $100-\mathrm{m}^{2}$ plot $(5 \mathrm{~m} \times 20 \mathrm{~m})$ centered within the $1000-\mathrm{m}^{2}$ plot, where species presence is recorded and within which $1-\mathrm{m}^{2}$ quadrats $(0.5 \mathrm{~m} \times 2.0 \mathrm{~m})$ are located inside and adjacent to the plot perimeter to measure abundance of species present

- a $10-\mathrm{m}^{2}$ plot $(2 \mathrm{~m} \times 5 \mathrm{~m})$ centered within the $100 \mathrm{~m}^{2}$ plot, where only species presence is recorded.

We modified this sampling design at several points to adjust the size and location of plots in order to stay within revegetation area boundaries. Where the revegetation area was large enough, replicate plots were established. Because the size and shape of each revegetated area is unique to the disturbance footprint, most sites were not large enough to accommodate the $1000-\mathrm{m}^{2}$ plot in the full modified Whittaker design described. The $1000-\mathrm{m}^{2}$ plot was set up at only two sites in Unit 2 and data are not reported for these larger plots. Some areas (such as roadways) were too narrow to accommodate a $5-\mathrm{m} \times 20-\mathrm{m}$ plot; in these cases, a narrower, longer rectangle of approximately $100 \mathrm{~m}^{2}$ was placed on the site. We then used $12,0.5-\mathrm{m} \times 1.0-\mathrm{m}\left(0.5 \mathrm{~m}^{2}\right)$ quadrats (instead of the $1-\mathrm{m}^{2}$ quadrats described above) to sample species abundance around the boundary of each plot (Figure 2.1). A few debris locations and small building footprint areas were too small to place a $100-\mathrm{m}^{2}$ plot, and these were sampled using perpendicular line transects with quadrats placed along the lines or randomly in equal number within each of the four quadrants created by the perpendicular transect layout.

All species present in both the 10 - and $100-\mathrm{m}^{2}$ plots were recorded to determine species richness. At two sampling sites where the areal extent of vegetation was large enough (the ALE Ecology Headquarters (HQ) site and the reference site in Unit 2), the species present within an additional $1000-\mathrm{m}^{2}$ plot were also recorded. Within each of the $100-\mathrm{m}^{2}$ plots, the heights of all large shrub species (big sagebrush (Artemisia tridentata), gray rabbitbrush (Ericameria nauseosa), and spiny hopsage (Grayia spinosa) were measured.

In each of the $0.5-\mathrm{m}^{2}$ quadrats, individual grass and forb plants were counted to measure the density of each species. Percent canopy cover and percent cover of bare ground and litter were also visually estimated. These values were used to calculate the mean percent canopy cover and mean density of both 
herbaceous species and short-statured woody plants such as thyme buckwheat (Eriogonum thymoides) and narrow-leaved goldenweed (Nestotus stenophyllus).

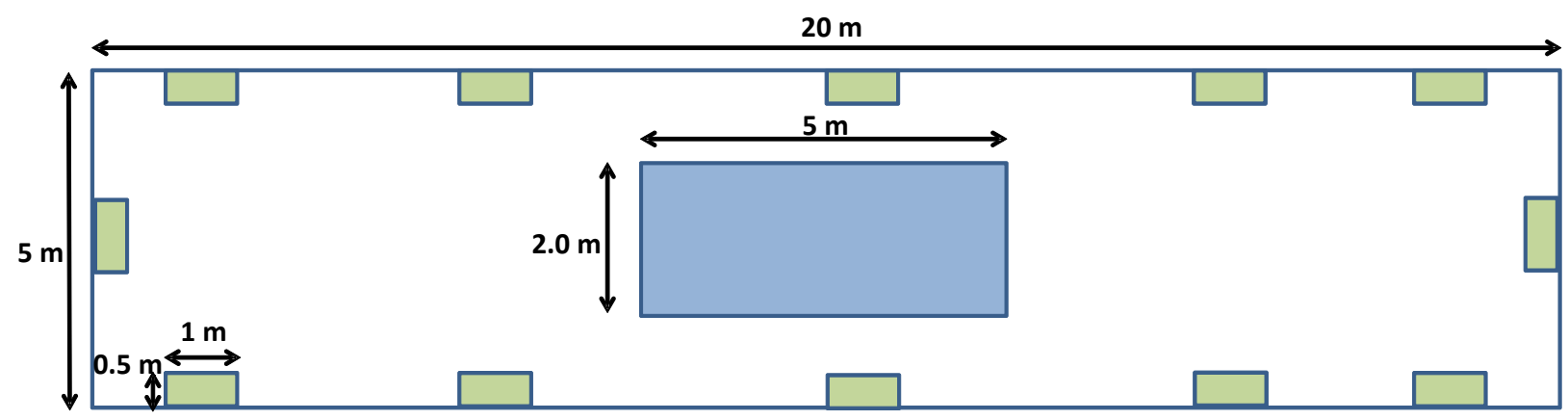

Figure 2.1. Diagram of Modified Whittaker Sample Plot Design. The $0.5-\mathrm{m} \times 1.0$-m quadrats were consistently placed along the meter tape, beginning at point A (lower left-hand corner of figure) in a counter-clockwise fashion.

Three sites were too small to place $100-\mathrm{m}^{2}$ modified Whittaker plots within the revegetated area. In these sampling areas, crossed transects (with length fit to the site area) were used, and $12,0.5-\mathrm{m}^{2}$ plots were placed at random along the crossed transect lines. Within the smallest sampling sites, species lists were recorded for a $1-\mathrm{m}^{2}$ plot and the entire revegetated area. Each plot was photographed, and the coordinates (Washington State Plane, South, $\mathrm{m}$ ) for each plot were recorded using a global positioning system unit to aid in relocating plots for future monitoring efforts.

A total of 33 sites on the revegetation units were sampled using these methods. In addition, modified Whittaker plots were established and monitored in two reference plant communities: one along the 1200-ft road in the bluebunch wheatgrass (Pseudoroegneria spicata)/Sandberg's bluegrass (Poa secunda) association, and one along the ridgetop in a thyme buckwheat-rock buckwheat (Eriogonum sphaerocephalum)/bluebunch wheatgrass cover type. The coordinates, transect bearing, size, and number of sampling plots and transects that were established in each revegetation unit are listed in Appendix B of this document. Appendix B also provides photographs of each of the $100-\mathrm{m}^{2}$ plots that were sampled.

\subsubsection{Monitoring Strategy for CTUIR Diversity Plantings}

Field monitoring occurred between June 17 and July 14, 2011. In the transplanted plots, all individuals of all species were counted and condition noted (green and growing, stressed, dead, flowering status). All seedlings of applied species were counted and condition noted. Enumeration was done by dividing a plot into small sections to facilitate accurate counts. Percent cover of each species and that of litter, soil, soil cryptogams, and rocks was estimated for each plot. Two observers were used for every plot. Photographs were taken of each plot after assessment.

\subsection{Data Summaries}

Data from modified Whittaker plots on individual subunits were summarized to provide information on the canopy cover of exotic species, the density of native grasses, forbs and shrubs, and the total and native species richness of vegetation that established in the first year on each revegetation unit. 
Additional summaries were generated to evaluate the individual sites or subunits within the unit that received similar seed mixes and treatments. Data tables for these measurements by species are included in Appendix $\mathrm{C}$ of this document.

Data collected on the diversity plots and circles planted or seeded by CTUIR were summarized to evaluate survivorship, which equaled the number of live seedlings divided by the number planted in each plot. Percent establishment for seeded species was determined by dividing the observed number of individuals of each species by the number of seed applied. Percent of plants in each condition class was also computed. Data were analyzed using JMP software (SAS Institute 2002) to compute means and one standard error of the mean. Data tables for these measurements are included in Appendix C. Additional information regarding CTUIR technical support is included in Appendix D of this document. 


\subsection{Revegetation Status and Initial Monitoring Results}

Results of the initial monitoring indicate that native plants were successfully established on target revegetation areas. In general, the initial target criteria for revegetation were met for most of the individual metrics at the majority of the sites. The following sections describe the areas within the revegetation units, the specific revegetation strategies implemented at each site, summarize the results of the initial monitoring of vegetation establishment in 2011 and compare results to the initial criteria listed in the revegetation plan for these areas (Downs et al. 2011). The mean values for native species richness, and the mean canopy cover and density of large shrubs, grasses and forbs are listed in Table 3.1 for each of the revegetation units.

Table 3.1. Summary of Native Species Density and Richness and Exotic Species Cover Measured in 2011 on ALE Revegetation Areas and on Two Nearby Reference Areas. Initial criteria are shown in parentheses following each measured element.

\begin{tabular}{|c|c|c|c|c|c|c|}
\hline $\begin{array}{l}\text { Revegetation } \\
\text { Unit }\end{array}$ & $\begin{array}{l}\text { Desired Future Plant } \\
\text { Community }\end{array}$ & $\begin{array}{l}\text { Native Shrub } \\
\text { Density } \\
\text { (plants/ } \\
100 \mathrm{~m}^{2} \text { ) }\end{array}$ & $\begin{array}{c}\text { Native } \\
\text { Bunchgrass } \\
\text { Density } \\
\text { (plants } / \mathrm{m}^{2} \text { ) }\end{array}$ & $\begin{array}{l}\text { Native Forb } \\
\text { Density } \\
\text { (plants } / \mathrm{m}^{2} \text { ) }\end{array}$ & $\begin{array}{l}\text { Native Species } \\
\text { Richness } \\
\text { (species/100 } \mathrm{m}^{2} \text { ) }\end{array}$ & $\begin{array}{c}\text { Exotic } \\
\text { Species } \\
\text { Cover } \\
(\%)\end{array}$ \\
\hline 1 & Black Greasewood/Saltgrass & $22(5)$ & 29 (15) & $1(1)$ & $13(4)$ & $\begin{array}{c}11 \\
(<30)\end{array}$ \\
\hline 2 & $\begin{array}{l}\text { Big Sagebrush/Bluebunch } \\
\text { Wheatgrass }\end{array}$ & $15(5)$ & $47(15)$ & $5(1)$ & $11(4)$ & $\begin{array}{l}20.8 \\
(<30)\end{array}$ \\
\hline 3 & $\begin{array}{l}\text { Thyme Buckwheat/ } \\
\text { Bluebunch Wheatgrass and } \\
\text { Big Sagebrush/Bluebunch } \\
\text { Wheatgrass-Idaho Fescue }\end{array}$ & $2(5)$ & $105(20)$ & $1(2)$ & $19(4)$ & $\begin{array}{c}8 \\
(<30)\end{array}$ \\
\hline 4 & $\begin{array}{l}\text { Thyme Buckwheat/ } \\
\text { Bluebunch Wheatgrass and } \\
\text { Big Sagebrush/Bluebunch } \\
\text { Wheatgrass-Idaho Fescue }\end{array}$ & $0(5)$ & $87(12)$ & $5(1)$ & $15(4)$ & $\begin{array}{c}9 \\
(<30)\end{array}$ \\
\hline 5 & $\begin{array}{l}\text { Thyme Buckwheat/ } \\
\text { Bluebunch Wheatgrass }\end{array}$ & $0(2)$ & $45(5)$ & $1(1)$ & $6(4)$ & $\begin{array}{c}0.1 \\
(<30)\end{array}$ \\
\hline 6 & $\begin{array}{l}\text { Thyme Buckwheat/ } \\
\text { Bluebunch Wheatgrass }\end{array}$ & $0(5)$ & $47(20)$ & $11(2)$ & $24(4)$ & $\begin{array}{c}40 \\
(<30)\end{array}$ \\
\hline 7 & $\begin{array}{l}\text { Eastside Canyon Shrublands } \\
\text { Transition }\end{array}$ & $3(5)$ & $5(20)$ & $3(2)$ & $16(4)$ & $\begin{array}{c}48 \\
(<30)\end{array}$ \\
\hline Reference 1 & $\begin{array}{l}\text { Big Sagebrush/Bluebunch } \\
\text { Wheatgrass }\end{array}$ & $1^{(\mathrm{a})}$ & 48 & 17 & 14 & 2.8 \\
\hline Reference 2 & $\begin{array}{l}\text { Thyme } \\
\text { Buckwheat/Bluebunch } \\
\text { Wheatgrass }\end{array}$ & 14 & 83 & 37 & 18 & 0.7 \\
\hline
\end{tabular}

(a) The reference site along the 1200-ft road on ALE has been burned previously and shrub densities are lower than desired target criteria for restoration 


\subsection{Unit 1}

Unit 1 consists of a single site associated with decommissioning and removal of the 646 Aquatic Research Laboratory. The site is near Rattlesnake Springs adjacent to a black greasewood/saltgrass plant community growing on fine-grained alkaline soils. The unit includes the building footprint, parking area and a short roadway.

\subsubsection{Unit 1 Planting Details}

The roadway and disturbance footprint in this unit were prepared by ripping with an s-tine harrow. Bunchgrasses were seeded with a rangeland drill and harrowed to incorporate seed into the soil. Forbs and Wyoming big sagebrush were then broadcast seeded across the entire unit. Several forb, shrub, and one vine species were broadcast seeded into three small areas to create islands of diversity within the disturbance footprint. Container-grown sagebrush and spiny hopsage were transplanted throughout the unit and two small patches were transplanted with two native forbs. Seeding and transplant details are provided in Table 3.2. Seeding rates and transplant densities are provided in Appendix A. A bottlebrush squirreltail (Elymus elymoides) native grass straw was applied across the unit and crimped in place to a depth of 3 to 4 in. Water was applied to the entire site at a nominal rate of 6000 gal/acre once planting activities were complete. Supplemental water was also provided to the spiny hopsage and transplanted forbs on April 18. In this case, each plant was watered by opening a hole next to the seedling with a spike and pouring approximately $250 \mathrm{ml}$ water into the hole.

Table 3.2. Seeding and Transplant Details for Unit 1

\begin{tabular}{|c|c|c|c|c|c|}
\hline Method & $\begin{array}{c}\text { Growth } \\
\text { Habit }\end{array}$ & Scientific Name & Common Name & $\begin{array}{c}\text { Whole } \\
\text { Unit }\end{array}$ & $\begin{array}{c}\text { Diversity } \\
\text { Islands }\end{array}$ \\
\hline \multirow{2}{*}{$\begin{array}{c}\text { drill } \\
\text { seeded }\end{array}$} & \multirow[t]{2}{*}{ bunchgrass } & Leymus cinereus & giant wildrye & $\mathrm{x}$ & \\
\hline & & Poa secunda & Sandberg's bluegrass & $\mathrm{x}$ & \\
\hline \multirow{11}{*}{$\begin{array}{l}\text { broadcast } \\
\text { seeded }\end{array}$} & \multirow[t]{8}{*}{ forb } & Achillea millefolium & yarrow & $\mathrm{x}$ & \\
\hline & & Astragalus succumbens ${ }^{(\mathrm{a})}$ & crouching milkvetch & & $\mathrm{x}$ \\
\hline & & Balsamorhiza careyana & Carey’s balsamroot & $\mathrm{x}$ & \\
\hline & & Chaenactis douglasii & hoary falseyarrow & & $\mathrm{x}$ \\
\hline & & Crepis atribarba & slender hawksbeard & & $\mathrm{x}$ \\
\hline & & Lomatium macrocarpum & bigseed desertparsley & $\mathrm{x}$ & \\
\hline & & Machaeranthera canescens & hoary aster & & $x$ \\
\hline & & Sphaeralcea munroana & Munro’s globemallow & $\mathrm{x}$ & \\
\hline & \multirow[t]{2}{*}{ shrub } & Artemisia tridentata & Wyoming big sagebrush & $\mathrm{x}$ & $x$ \\
\hline & & Grayia spinosa & spiny hopsage & & $\mathrm{x}$ \\
\hline & vine & Clematis ligusticifolia & western white clematis & & $\mathrm{x}$ \\
\hline \multirow[t]{4}{*}{ transplant } & \multirow[t]{2}{*}{ forb } & Agoseris grandiflora & showy mountain dandelion & & $\mathrm{x}$ \\
\hline & & Astragalus caricinus & buckwheat milkvetch & & $\mathrm{x}$ \\
\hline & \multirow[t]{2}{*}{ shrub } & Artemisia tridentata & Wyoming big sagebrush & $\mathrm{x}$ & \\
\hline & & Grayia spinosa & spiny hopsage & $\mathrm{x}$ & \\
\hline
\end{tabular}

(a) State Watch List species. 


\subsubsection{Unit 1 Vegetation Establishment}

Native perennial grass establishment on Unit 1 was dominated by bottlebrush squirreltail with a mean density of 21 plants $/ \mathrm{m}^{2}$. These plants established from seeds within the native bunchgrass straw that was crimped over the site after seeding. Sandberg's bluegrass, alkali saltgrass (Distichilis stricta) and Great Basin wildrye (Leymus cinerus) also established from seeding and existing propagules on the site. These four species contributed to a total mean native grass density of $29 \mathrm{plants} / \mathrm{m}^{2}$. Mean densities of big sagebrush and spiny hopsage were approximately 13 and 9 shrubs $/ 100 \mathrm{~m}^{2}$, respectively.

Only one native forb was rooted within the quadrats sampled at Unit 1; common yarrow (Achillea millefolium) was present at a density of about $1 \mathrm{plant} / 3 \mathrm{~m}^{2}$. Hoary aster (Machaeranthera canescens) was also present, but not rooted within the $0.5-\mathrm{m}^{2}$ quadrats. Three non-native summer annual forbs-Russian thistle (Salsola tragus), tumble mustard (Sisymbirum altissimum), and kochia or burning bush (Bassia scoparia) were common on the revegetation site.

\subsection{Unit 2}

Unit 2 consists of 10 subunits adjacent to the 1200-ft road. The 6652 ALE Ecology subunit is a 2-ac disturbance footprint associated with the removal and decommissioning of several buildings. The Hodges Well subunit is a 0.52 -ac footprint associated with well decommissioning, pumphouse removal, and a short roadway. The eight remaining subunits are made up of miscellaneous debris sites with a total area of 1.7 ac flanking the $1200-\mathrm{ft}$ road between 1000 and $1600 \mathrm{ft}$ elevation. Each of the subunits in Unit 2 is adjacent to a big sagebrush/bluebunch wheatgrass plant community growing on fine-grained silt-loam soils.

\subsubsection{Unit 2 Planting Details}

Planting methods varied depending on subunit size and access. Both the ALE Ecology and Hodges Well subunits were accessed by road, which facilitated the use of heavy equipment. The eight debris sites were either reached by foot or by light vehicle along primitive but well-vegetated two-track roads. Site preparation and seeding equipment used on the debris sites was limited to a rototiller, hand rakes, and hand-pushed seed broadcasters. Hoedads and dibble bars were used to install transplant stock at the Unit 2 locations. Preparation and planting activities performed at each subunit are described in the following subsections. Seeding rates and transplant densities are provided in Appendix A.

\subsubsection{ALE Ecology and Hodges Well Sub Units}

Both the ALE Ecology HQ (6652-H, I, and J building footprints) and Hodges Well subunits were prepared by ripping with an s-tine harrow. Bunchgrasses were seeded with a rangeland drill and harrowed to incorporate seed. Additional bunchgrasses, forbs, and shrubs were then broadcast seeded across each subunit. Eleven seeded islands (eight at the ALE subunit; three at Hodges Well), and six transplant islands (ALE subunit only) were created to encourage additional native diversity. Seeded islands were circular with varying radii from 2 to $2.6 \mathrm{~m}$ depending on the number of seeds planted. Transplant islands were circular with a 5-m radius. Container-grown sagebrush were transplanted throughout both subunits. Seeding and transplant details for the ALE Ecology HQ and Hodges Well subunits are provided in Table 3.3. Squirreltail straw was applied across both subunits and crimped into 
place to a depth of 3 to $4 \mathrm{in}$. Water was applied at a nominal rate of $6000 \mathrm{gal} / \mathrm{ac}$ once planting activities were complete. No additional supplemental water was provided.

Table 3.3. Seeding and Transplant Detail for the ALE Ecology and Hodges Well Subunits in Unit 2

\begin{tabular}{|c|c|c|c|c|c|}
\hline Method & $\begin{array}{c}\text { Growth } \\
\text { Habit }\end{array}$ & Scientific Name & Common Name & Subunit & $\begin{array}{c}\text { Diversity } \\
\text { Islands }\end{array}$ \\
\hline \multirow{2}{*}{$\begin{array}{c}\text { drill } \\
\text { seeded }\end{array}$} & \multirow[t]{2}{*}{ bunchgrass } & Pseudoroegneria spicata & bluebunch wheatgrass & $\mathrm{x}$ & \\
\hline & & Poa secunda & Sandberg's bluegrass & $\mathrm{x}$ & \\
\hline \multirow{16}{*}{$\begin{array}{l}\text { broadcast } \\
\text { seeded }\end{array}$} & \multirow[t]{2}{*}{ bunchgrass } & Achnatherum hymenoides & Indian ricegrass & $x^{(a)}$ & \\
\hline & & Elymus elymoides & bottlebrush squirreltail & $\mathrm{x}$ & \\
\hline & \multirow[t]{12}{*}{ forb } & Achillea millefolium & yarrow & $\mathrm{x}$ & \\
\hline & & Astragalus succumbens $^{(\mathrm{b})}$ & crouching milkvetch & & $\mathrm{x}$ \\
\hline & & Balsamorhiza careyana & Carey’s balsamroot & $\mathrm{x}$ & \\
\hline & & Calochortus macrocarpus & sagebrush mariposa lily & & $\mathrm{x}$ \\
\hline & & Chaenactis douglasii & hoary falseyarrow & & $\mathrm{x}$ \\
\hline & & Crepis atribarba & slender hawksbeard & $\mathrm{x}$ & $\mathrm{x}$ \\
\hline & & Erigeron filifolius & threadleaf fleabane & & $\mathrm{x}$ \\
\hline & & Erigeron piperianus $^{(\mathrm{c})}$ & Piper's daisy & & $\mathrm{x}$ \\
\hline & & Helianthus cusickii & Cusick's sunflower & & $\mathrm{x}$ \\
\hline & & Lomatium macrocarpum & bigseed desertparsley & $x^{(a)}$ & \\
\hline & & Lupinus sericeus & silky lupine & & $\mathrm{x}$ \\
\hline & & Machaeranthera canescens & hoary aster & $\mathrm{x}$ & $\mathrm{x}$ \\
\hline & \multirow[t]{2}{*}{ shrub } & Artemisia tridentata & Wyoming big sagebrush & $\mathrm{x}$ & \\
\hline & & Ericameria nauseosa & gray rabbitbrush & & $\mathrm{x}$ \\
\hline \multirow[t]{7}{*}{ transplants } & \multirow{6}{*}{$\begin{array}{l}\text { bunchgrass } \\
\text { forb }\end{array}$} & Achnatherum thurberianum & Thurber's needlegrass & & $\mathrm{x}^{(\mathrm{d})}$ \\
\hline & & Agoseris grandiflora & showy mountain dandelion & & $\mathrm{x}^{(\mathrm{d})}$ \\
\hline & & Astragalus caricinus & buckwheat milkvetch & & $x^{(d)}$ \\
\hline & & Erigeron piperianus $^{(\mathrm{c})}$ & Piper’s daisy & & $\mathrm{x}^{(\mathrm{d})}$ \\
\hline & & Lupinus sericeus & silky lupine & & $x^{(d)}$ \\
\hline & & Phlox longifolia & longleaf phlox & & $\mathrm{x}^{(\mathrm{d})}$ \\
\hline & shrub & Artemisia tridentata & Wyoming big sagebrush & $\mathrm{x}$ & \\
\hline $\begin{array}{l}\text { (a) Hodges } \\
\text { (b) State W } \\
\text { (c) State Se } \\
\text { (d) ALE Ec }\end{array}$ & $\begin{array}{l}\text { Well subunit } \\
\text { atch List spe } \\
\text { nsitive speci } \\
\text { ology subuni }\end{array}$ & & & & \\
\hline
\end{tabular}

\subsubsection{Debris Site Sub Units}

Five debris sites were planted with a similar suite of forbs and shrubs. Site 50 is about 0.4 ac including a small disturbance footprint associated with cleanup and a well-vegetated roadway leading to the site. Site 109 is associated with a disturbance footprint from the removal of gas wells at two small pads (about $0.03 \mathrm{ac}$ ), a 0.13 -ac contractor turnaround, and a 1.2-ac section of primitive but well-vegetated road. Site 60 is made up of two small footprints totaling $0.05 \mathrm{ac}$ and site 146, located below the 1200-ft road, includes a small 0.07 -ac footprint associated with debris cleanup. Each site (with the exception of 
the road portions described) was rototilled prior to seeding with bunchgrasses. Hand rakes were used to incorporate seeds. Forbs and sagebrush seed were then hand broadcast and native grass straw composed of Idaho fescue (Festuca idahoensis) was distributed atop each of the planting areas. Squirreltail straw was applied to the upper planting site at subunit 60 . The straw was not crimped and supplemental water was not provided.

Seven seeded islands (one each at subunits 50,109, and 138; two each at subunits 60 and 146) were created to increase native diversity. Seeded islands were circular with varying radii from 2 to $2.6 \mathrm{~m}$ depending on the number of seeds planted. Sagebrush were transplanted along the roads on subunits 50 and 109. Seeding and transplant details for these subunits are provided in Table 3.4.

Table 3.4. Seeding and Transplant Detail for Debris Sites 50, 60, 109, 138, and 146

\begin{tabular}{|c|c|c|c|c|c|}
\hline Method & $\begin{array}{c}\text { Growth } \\
\text { Habit }\end{array}$ & Scientific Name & Common Name & Subunit & $\begin{array}{c}\text { Diversity } \\
\text { Islands }\end{array}$ \\
\hline \multirow{17}{*}{$\begin{array}{c}\text { broadcast } \\
\text { seeded }\end{array}$} & \multirow[t]{2}{*}{ bunchgrass } & Pseudoroegneria spicata & bluebunch wheatgrass & $\mathrm{x}$ & \\
\hline & & Poa secunda & Sandberg's bluegrass & $\mathrm{x}$ & \\
\hline & \multirow[t]{13}{*}{ forb } & Achillea millefolium & yarrow & $\mathrm{x}$ & \\
\hline & & Astragalus caricinus & buckwheat milkvetch & & $x^{(a)}$ \\
\hline & & Astragalus succumbens $^{(\mathrm{b})}$ & crouching milkvetch & & $\mathrm{x}$ \\
\hline & & Balsamorhiza careyana & Carey's balsamroot & $\mathrm{x}$ & \\
\hline & & Calochortus macrocarpus & sagebrush mariposa lily & & $\mathrm{x}$ \\
\hline & & Chaenactis douglasii & hoary falseyarrow & & $\mathrm{x}$ \\
\hline & & Crepis atribarba & slender hawksbeard & & $\mathrm{x}$ \\
\hline & & Erigeron filifolius & threadleaf fleabane & & $\mathrm{x}$ \\
\hline & & Erigeron piperianus $^{(\mathrm{c})}$ & Piper's daisy & & $\mathrm{x}$ \\
\hline & & Helianthus cusickii & Cusick's sunflower & & $\mathrm{x}$ \\
\hline & & Lomatium macrocarpum & bigseed desertparsley & $\mathrm{x}$ & \\
\hline & & Lupinus sericeus & silky lupine & & $\mathrm{x}$ \\
\hline & & Machaeranthera canescens & hoary aster & & $\mathrm{x}$ \\
\hline & \multirow[t]{2}{*}{ shrub } & Artemisia tridentata & Wyoming big sagebrush & $\mathrm{x}$ & \\
\hline & & Ericameria nauseosa & gray rabbitbrush & & $\mathrm{x}$ \\
\hline transplants & shrub & Artemisia tridentata & Wyoming big sagebrush & $\mathrm{x}$ & $\mathrm{x}$ \\
\hline \multicolumn{6}{|c|}{ (a) Debris-site 60 only. } \\
\hline \multicolumn{6}{|c|}{ (b) State Watch List species. } \\
\hline \multicolumn{6}{|c|}{ (c) State Sensitive species. } \\
\hline
\end{tabular}

Debris site 40, 127, and 139 were the smallest of the subunits, ranging in size from 0.01 to 0.02 ac. Diversity islands were not created on these smaller sites. Subunits 127 and 139 were rototilled prior to broadcast seeding with bunchgrasses. Hand rakes were used to incorporate seeds. Forbs and sagebrush seed were then hand broadcast. Idaho fescue straw was distributed atop each planting area but was not crimped into place. Sagebrush transplants were installed on debris sites 40 and 139 only. Seeding and transplant details for these subunits are provided in Table 3.5. Supplemental water was not applied. 
Table 3.5. Seeding and Transplanting Detail for Debris Sites $40,{ }^{(a)} 127$, and 139

\begin{tabular}{|c|c|c|c|c|c|}
\hline Method & $\begin{array}{c}\text { Growth } \\
\text { Habit }\end{array}$ & Scientific Name & Common Name & $\begin{array}{l}\text { Sub } \\
\text { Unit }\end{array}$ & $\begin{array}{c}\text { Diversity } \\
\text { Islands }\end{array}$ \\
\hline \multirow{6}{*}{$\begin{array}{c}\text { broadcast } \\
\text { seeded }\end{array}$} & bunchgrass & Pseudoroegneria spicata & bluebunch wheatgrass & $\mathrm{x}$ & \\
\hline & & Poa secunda & Sandberg's bluegrass & $\mathrm{x}$ & \\
\hline & forb & Achillea millefolium & yarrow & $\mathrm{x}$ & \\
\hline & & Balsamorhiza careyana & Carey’s balsamroot & $\mathrm{x}$ & \\
\hline & & Lomatium macrocarpum & bigseed desertparsley & $\mathrm{x}$ & \\
\hline & shrub & Artemisia tridentata & Wyoming big sagebrush & $\mathrm{x}$ & \\
\hline transplants & shrub & Artemisia tridentata & Wyoming big sagebrush & $\mathrm{x}^{(\mathrm{b})}$ & \\
\hline
\end{tabular}

(a) Debris site 40 received only sagebrush transplants.

(b) Debris sites 40 and 139 only.

\subsubsection{Unit 2 Vegetation Establishment}

Native perennial grass establishment on Unit 2 was dominated by bluebunch wheatgrass, Sandberg's bluegrass, and bottlebrush squirreltail with a mean density of 21 plants $/ \mathrm{m}^{2}$. Table 3.6 provides a summary of the attributes measured on seven of the subunits within Unit 2. Native bunchgrass mean density measured on the subunits ranged from 7 to 140 plants $/ \mathrm{m}^{2}$, and exotic species mean canopy cover measured on the subunits ranged from $2.5 \%$ to $58 \%$. Exotic species cover exceeded initial target criteria within several of the unit 2 subunits. Russian thistle (Salsola kali) was the largest contributor to exotic species cover on the ALE Ecology HQ plots and subunits on debris areas 138 and 139; whereas cheatgrass (Bromus tectorum) was the major contributor to exotic species cover on the 109 debris site. High values for exotic species cover on the Hodges Well subunit were due to several summer annual exotic forbs, but Jim Hill mustard (Sisymbrium altissumumi) was the primary exotic species.

Table 3.6. Initial Monitoring Results for Vegetation Characteristics Measured on Subunits within Unit 2

\begin{tabular}{lccccc}
\hline $\begin{array}{c}\text { Revegetation } \\
\text { Subunit }\end{array}$ & $\begin{array}{c}\text { Mean Native } \\
\text { Shrub Density } \\
\left(\text { plants } / 100 \mathrm{~m}^{2}\right)\end{array}$ & $\begin{array}{c}\text { Mean Native } \\
\text { Bunchgrass } \\
\text { Density } \\
\left(\text { plants } / \mathrm{m}^{2}\right)\end{array}$ & $\begin{array}{c}\text { Mean Native } \\
\text { Forb Density } \\
\left(\mathrm{plants} / \mathrm{m}^{2}\right)\end{array}$ & $\begin{array}{c}\text { Native Species } \\
\text { Richness } \\
\left(\text { species } / 100 \mathrm{~m}^{2}\right)\end{array}$ & $\begin{array}{c}\text { Mean Exotic } \\
\text { Species Cover } \\
(\%)\end{array}$ \\
\hline 2_109 & 9 & 30 & 12 & 14 & 16 \\
2_138 and 139 & 12.5 & 9 & 3 & 6 & 32 \\
2_50 & 10 & 70.8 & 8 & 18 & 3 \\
2_60 & 14.5 & 42 & 6 & 14 & 5 \\
2_146 & 49 & 29 & 10 & 15 & 45 \\
2_HQ & 14 & 93 & 2 & 13 & 14 \\
2_Hodges Well & 7.5 & 115 & 12 & 10 & 38 \\
\hline
\end{tabular}

\subsection{Unit 3}

Unit 3 consists of a single 0.36 -ac site associated with decommissioning and removal of the 6631 Radiotelescope and roadway. The unit is located along Rattlesnake Ridge at an elevation of approximately $3000 \mathrm{ft}$. Adjacent vegetation grades from elements of lithosolic plant communities of 
thyme buckwheat or roundhead buckwheat and bluebunch wheatgrass into a bunchgrass and shrub community containing bluebunch wheatgrass, Idaho fescue, big sagebrush, green rabbitbrush (Chrysothamnus viscidiflorus), gray rabbitbrush, and three-tip sagebrush (Artemisia tripartita). The substrate of Unit 3 is of thin compacted soils with some rocky/gravel surfaces.

\subsubsection{Unit 3 Planting Details}

The roadway and disturbance footprint were prepared by ripping with an s-tine harrow. Bunchgrasses were broadcast seeded and harrowed to incorporate seed into the soil. Thyme buckwheat seed was then broadcast. Two diversity islands, one seeded and one transplant, were created to encourage additional native diversity within the disturbance footprint. The seeded island was installed by broadcasting seed within a 1-m radius. The transplant island was circular with a 4-m radius. Transplants were installed using pry bars, and rocks were placed in a circle around the plants, especially on the upwind side of the seedling, to create a protected space. A guar-gum tackifier (Super Tack ${ }^{\circledR}$ ) was applied at a rate of $50-1 \mathrm{~b} / \mathrm{ac}$ ) with supplemental watering applied at a nominal rate of $6000 \mathrm{gal} / \mathrm{ac}$ across the entire unit. Seeding and transplant details for this unit are provided in Table 3.7. Seeding and transplant rates are provided in Appendix A.

Table 3.7. Seeding and Transplant Details for Unit 3

\begin{tabular}{|c|c|c|c|c|c|}
\hline Method & $\begin{array}{c}\text { Growth } \\
\text { Habit }\end{array}$ & Scientific Name & Common Name & $\begin{array}{c}\text { Whole } \\
\text { Unit }\end{array}$ & $\begin{array}{c}\text { Diversity } \\
\text { Islands }\end{array}$ \\
\hline \multirow{11}{*}{$\begin{array}{l}\text { broadcast } \\
\text { seeded }\end{array}$} & \multirow[t]{3}{*}{ bunchgrass } & Festuca idahoensis & Idaho fescue & $\mathrm{x}$ & \\
\hline & & Pseudoroegneria spicata & bluebunch wheatgrass & $\mathrm{x}$ & \\
\hline & & Poa secunda & Sandberg's bluegrass & $\mathrm{x}$ & \\
\hline & \multirow[t]{4}{*}{ forb } & Agastache occidentalis & western horsemint & & $\mathrm{x}$ \\
\hline & & Astragalus purshii & woolly-pod milkvetch & & $\mathrm{x}$ \\
\hline & & Lomatium macrocarpum & bigseed desertparsley & & $\mathrm{x}$ \\
\hline & & Lupinus sericeus & silky lupine & & $\mathrm{x}$ \\
\hline & \multirow[t]{2}{*}{ shrub } & Artemisia tridentata & Wyoming big sagebrush & & $\mathrm{x}$ \\
\hline & & Ericameria nauseosa & gray rabbitbrush & & $\mathrm{x}$ \\
\hline & \multirow[t]{2}{*}{ subshrub } & Eriogonum sphaerocephalum & rock buckwheat & & $\mathrm{x}$ \\
\hline & & Eriogonum thymoides & thymeleaf buckwheat & $\mathrm{x}$ & $\mathrm{x}$ \\
\hline \multirow[t]{13}{*}{ transplants } & \multirow[t]{9}{*}{ forb } & Agastache occidentalis & western horsemint & & $\mathrm{x}$ \\
\hline & & Astragalus purshii & woolly-pod milkvetch & & $\mathrm{x}$ \\
\hline & & Balsamorhiza rosea $^{(\mathrm{a})}$ & rosy balsamroot & & $\mathrm{x}$ \\
\hline & & Erigeron linearis & desert yellowdaisy & & $\mathrm{x}$ \\
\hline & & Erigeron poliospermus & cushion fleabane & & $\mathrm{x}$ \\
\hline & & Penstemon speciosus & showy beardtongue & & $\mathrm{x}$ \\
\hline & & Phemeranthus spinescens & spiny flameflower & & $\mathrm{x}$ \\
\hline & & Phlox hoodii & Hood's phlox & & $\mathrm{x}$ \\
\hline & & Sedum leibergii & Leiberg's stonecrop & & $\mathrm{x}$ \\
\hline & \multirow{4}{*}{$\begin{array}{l}\text { shrub } \\
\text { subshrub }\end{array}$} & Salvia dorrii & grayball sage & & $\mathrm{x}$ \\
\hline & & Eriogonum sphaerocephalum & rock buckwheat & & $\mathrm{x}$ \\
\hline & & Eriogonum thymoides & thymeleaf buckwheat & & $\mathrm{x}$ \\
\hline & & Nestotus stenophyllus & narrowleaf goldenweed & & $\mathrm{x}$ \\
\hline
\end{tabular}

(a) State Watch List species. 


\subsubsection{Unit 3 Vegetation Establishment}

Vegetation establishment on the radiotelescope site was dominated by native perennial grasses ( $\sim 29 \%$ canopy cover). Bluebunch wheatgrass and Sandberg's bluegrass were the primary contributors with cover of $17 \%$ and $10 \%$, and average densities of 45 and 46 plants $/ \mathrm{m}^{2}$, respectively. Exotic species cover (Table 3.1) on Unit 3 was below initial target criteria.

\subsection{Unit 4}

Unit 4 consists of four subunits associated with the removal of buildings and associated infrastructure, and the disturbance footprint from construction of a new communication facility located at the uppermost elevations along Rattlesnake Ridge. The surrounding native plant communities consist primarily of a thyme buckwheat/bluebunch wheatgrass association that transitions to a big sagebrush/bluebunch wheatgrass/Idaho fescue association where pockets of deeper soils occur. Soils are shallow stony silt loams. Substrate conditions after building removal and other construction consist of stony loam soils with rocks and rubble.

\subsubsection{Unit 4 Planting Details}

Unit 4 subunits include the 623A Building footprint and roadway; the 6652-C building footprint; the Combined Community Communications Facility (CCCF) construction footprint; and a linear area associated with the removal of an overhead powerline and construction of a new underground power service. Diversity islands were created by seeding and transplanting on most of the subunits. Seeded diversity islands were broadcast seeded into circular areas with radii ranging from 0.5 to $1.4 \mathrm{~m}$ depending on the number of seeds planted. Transplant islands were circular with a 4-m radius except where circumstances required planting in other irregular patterns. Rocks were circled around each transplant, especially on the upwind side, to create a protected space for the seedlings. Preparation and planting activities performed at each subunit are described in the following subsections. Seeding rates and transplant densities are provided in Appendix A.

\subsubsection{623A Subunit}

The roadway and disturbance footprint associated with building removal were prepared by ripping with an s-tine harrow. Bunchgrasses were broadcast seeded and harrowed to incorporate seed into the soil. Thyme buckwheat seed was then broadcast. Super Tack ${ }^{\circledR}$ was applied (50-lb/ac) with supplemental watering at a nominal rate of $6000 \mathrm{gal} / \mathrm{ac}$ across the entire sub unit. No diversity islands were created. Seeding details are provided in Table 3.8 .

Table 3.8. Seeding Detail for Unit 4 (Subunit 623A)

\begin{tabular}{ccllcc}
\hline Method & Growth & \multicolumn{1}{c}{ Scientific Name } & \multicolumn{1}{c}{ Common Name } & Subunit & $\begin{array}{c}\text { Diversity } \\
\text { Islands }\end{array}$ \\
\hline broadcast & bunchgrass & Festuca idahoensis & Idaho fescue & $\mathrm{x}$ & \\
seeded & & Pseudoroegneria spicata & bluebunch wheatgrass & $\mathrm{x}$ \\
& & Poa secunda & Sandberg's bluegrass & $\mathrm{x}$ \\
& subshrub & Eriogonum thymoides & thymeleaf buckwheat & $\mathrm{x}$ \\
\hline
\end{tabular}




\subsubsection{6652-C Building Subunit}

The disturbance footprint associated with the removal of buildings, sidewalks, and a two-track roadway were prepared by ripping with an s-tine harrow. Bunchgrasses were broadcast seeded and harrowed to incorporate seed into the rocky soil. Thyme buckwheat seed was then broadcast. Seven seeded islands and six transplant islands were created as previously described to increase native diversity. Seeding and transplant details are provided in Table 3.9. Super Tack $^{\circledR}$ was applied $(50-1 \mathrm{~b} / \mathrm{ac})$ with supplemental watering at a nominal rate of $6000 \mathrm{gal} / \mathrm{ac}$ across the entire subunit.

Table 3.9. Seeding and Transplant Details for Unit 4 (Subunit 6652-C)

\begin{tabular}{|c|c|c|c|c|c|}
\hline Method & $\begin{array}{c}\text { Growth } \\
\text { Habit }\end{array}$ & Scientific Name & Common Name & $\begin{array}{c}\text { Whole } \\
\text { Unit }\end{array}$ & $\begin{array}{c}\text { Diversity } \\
\text { Islands }\end{array}$ \\
\hline \multirow{19}{*}{$\begin{array}{l}\text { broadcast } \\
\text { seeded }\end{array}$} & \multirow[t]{3}{*}{ bunchgrass } & Festuca idahoensis & Idaho fescue & $\mathrm{x}$ & \\
\hline & & Pseudoroegneria spicata & bluebunch wheatgrass & $\mathrm{x}$ & \\
\hline & & Poa secunda & Sandberg's bluegrass & $\mathrm{x}$ & \\
\hline & \multirow[t]{11}{*}{ forb } & Agastache occidentalis & western horsemint & & $\mathrm{x}$ \\
\hline & & $\begin{array}{l}\text { Astragalus conjunctus var. } \\
\text { rickardii }^{\text {(a) }}\end{array}$ & Dr. Bills locoweed & & $\mathrm{x}$ \\
\hline & & Astragalus purshii & woolly-pod milkvetch & & $\mathrm{x}$ \\
\hline & & Balsamorhiza rosea ${ }^{(a)}$ & rosy balsamroot & & $\mathrm{x}$ \\
\hline & & Erigeron linearis & desert yellowdaisy & & $\mathrm{x}$ \\
\hline & & Erigeron poliospermus & cushion fleabane & & $\mathrm{x}$ \\
\hline & & $\begin{array}{l}\text { Eriophyllum lanatum var. } \\
\text { integrifolum }\end{array}$ & woolly sunflower & & $\mathrm{x}$ \\
\hline & & Lomatium grayi & Gray's desertparsley & & $\mathrm{x}$ \\
\hline & & Penstemon speciosus & showy beardtongue & & $\mathrm{x}$ \\
\hline & & Phemeranthus spinescens & spiny flameflower & & $\mathrm{x}$ \\
\hline & & Sedum leibergii & Leiberg's stonecrop & & $\mathrm{x}$ \\
\hline & \multirow[t]{2}{*}{ shrub } & Salvia dorrii & grayball sage & & $\mathrm{x}$ \\
\hline & & Tetradymia canescens & gray horsebrush & & $\mathrm{x}$ \\
\hline & \multirow[t]{3}{*}{ subshrub } & Eriogonum sphaerocephalum & rock buckwheat & & $\mathrm{x}$ \\
\hline & & Eriogonum thymoides & thymeleaf buckwheat & $\mathrm{x}$ & \\
\hline & & Nestotus stenophyllus & narrowleaf goldenweed & & $\mathrm{x}$ \\
\hline \multirow[t]{14}{*}{ transplant } & \multirow[t]{10}{*}{ forb } & Agastache occidentalis & western horsemint & & $\mathrm{x}$ \\
\hline & & Astragalus purshii & woolly-pod milkvetch & & $\mathrm{x}$ \\
\hline & & Balsamorhiza rosea $^{(\mathrm{a})}$ & rosy balsamroot & & $\mathrm{x}$ \\
\hline & & Crepis modocensis & low hawksbeard & & $\mathrm{x}$ \\
\hline & & Erigeron linearis & desert yellowdaisy & & $\mathrm{x}$ \\
\hline & & Erigeron poliospermus & cushion fleabane & & $\mathrm{x}$ \\
\hline & & Penstemon speciosus & showy beardtongue & & $\mathrm{x}$ \\
\hline & & Phemeranthus spinescens & spiny flameflower & & $\mathrm{x}$ \\
\hline & & Phlox hoodii & Hood's phlox & & $\mathrm{x}$ \\
\hline & & Sedum leibergii & Leiberg's stonecrop & & $\mathrm{x}$ \\
\hline & \multirow{4}{*}{$\begin{array}{l}\text { shrub } \\
\text { subshrub }\end{array}$} & Salvia dorrii & grayball sage & & $\mathrm{x}$ \\
\hline & & Eriogonum sphaerocephalum & rock buckwheat & & $\mathrm{x}$ \\
\hline & & Eriogonum thymoides & thymeleaf buckwheat & & $\mathrm{x}$ \\
\hline & & Nestotus stenophyllus & narrowleaf goldenweed & & $\mathrm{x}$ \\
\hline
\end{tabular}

(a) State Watch List species. 


\subsubsection{CCCF Subunit}

The CCCF construction footprint includes some off-road damage due east and due south of the new facility, a roadway leading behind the site to the east and north, and several new berms surrounding the new structures. Level areas were prepared by ripping with an s-tine harrow. Bunchgrasses were broadcast seeded across the entire unit, including new berms. The level areas were then harrowed to incorporate seed. Thyme buckwheat seed was then hand broadcast. Five seeded diversity islands were created in the off-road damage area south of the new facility. Super Tack ${ }^{\circledR}$ was applied (50-lb/ac) with supplemental watering at a nominal rate of $6000 \mathrm{gal} / \mathrm{ac}$ across the entire sub unit. No transplant islands were created. Seeding details are provided in Table 3.10.

Table 3.10. Seeding and Transplant Detail for Unit 4 (Subunit CCCF)

\begin{tabular}{|c|c|c|c|c|c|}
\hline Method & $\begin{array}{c}\text { Growth } \\
\text { Habit }\end{array}$ & Scientific Name & Common Name & $\begin{array}{l}\text { Sub } \\
\text { Unit }\end{array}$ & $\begin{array}{l}\text { Diversity } \\
\text { Islands }\end{array}$ \\
\hline \multirow{16}{*}{$\begin{array}{c}\text { broadcast } \\
\text { seeded }\end{array}$} & bunchgrass & Festuca idahoensis & Idaho fescue & $\mathrm{x}$ & \\
\hline & & Pseudoroegneria spicata & bluebunch wheatgrass & $\mathrm{x}$ & \\
\hline & & Poa secunda & Sandberg's bluegrass & $\mathrm{x}$ & \\
\hline & forb & Agastache occidentalis & western horsemint & & $\mathrm{x}$ \\
\hline & & Astragalus purshii & woolly-pod milkvetch & & $\mathrm{x}$ \\
\hline & & Balsamorhiza rosea $^{(\mathrm{a})}$ & rosy balsamroot & & $\mathrm{x}$ \\
\hline & & Erigeron linearis & desert yellowdaisy & & $\mathrm{x}$ \\
\hline & & Erigeron poliospermus & cushion fleabane & & $\mathrm{x}$ \\
\hline & & $\begin{array}{l}\text { Eriophyllum lanatum var. } \\
\text { integrifolum }\end{array}$ & woolly sunflower & & $\mathrm{x}$ \\
\hline & & Lomatium grayi & Gray's desertparsley & & $\mathrm{x}$ \\
\hline & & Lomatium triternatum & nineleaf desertparsley & & $\mathrm{x}$ \\
\hline & & Sedum leibergii & Leiberg's stonecrop & & $\mathrm{x}$ \\
\hline & shrub & Tetradymia canescens & gray horsebrush & & $\mathrm{x}$ \\
\hline & subshrub & Eriogonum sphaerocephalum & rock buckwheat & & $\mathrm{x}$ \\
\hline & & Eriogonum thymoides & thymeleaf buckwheat & $\mathrm{x}$ & \\
\hline & & Nestotus stenophyllus & narrowleaf goldenweed & & $\mathrm{x}$ \\
\hline
\end{tabular}

(a) State Watch List species.

\subsubsection{Powerline Roadway Subunit}

Re-contouring the powerline road along the northeast face ridge has resulted in the invasion of significant numbers of cheatgrass. The population was documented and seed removal attempted by mechanical means and by hand. Cheatgrass control was initiated on June 15 and finished on August 2, 2011. Plants were cut with a gasoline powered Honda weed eater or pulled by hand. Plants and seed were picked up using a Stihl vacuum along with rakes and hand picking. Trash bags were filled with the material and removed from the area. No seeding or planting was conducted on this subunit in 2011. 


\subsubsection{Unit 4 Vegetation Establishment}

Native bunchgrass density on subunit $623 \mathrm{~A}$ was approximately 8 plants $/ \mathrm{m}^{2}$ and the major contributor was Sandberg's bluegrass. Native forb density on this subunit totaled just slightly less than $1 \mathrm{plant} / \mathrm{m}^{2}$. Exotic cover on subunit $623 \mathrm{~A}$ was measured at approximately $6 \%$, with nearly equal amounts of Russian thisle and cheatgrass ( $\sim 5 \%$ each) occurring on the site. Total species richness on this subunit was 10 with 6 native species occurring within the $100-\mathrm{m}^{2}$ plots.

Native bunchgrass densities in Unit 4 were greatest on the 6652-C subunit and totaled almost 200 individuals $/ \mathrm{m}^{2}$. Sandberg's bluegrass was the primary contributor to native bunchgrass density (150 plants $/ \mathrm{m}^{2}$ ) co-occurring with bluebunch wheatgrass, Idaho fescue, and bottlebrush squirreltail. The native forb, white scorpionweed (Phacelia hastata) appeared to act as a pioneer species and occurred at densities of 8 plants $/ \mathrm{m}^{2}$ on the 6652-C Building subunit. Invasive species canopy cover was less than $14 \%$ and was dominated by Russian thistle and Jim Hill mustard. Native species richness was above 8 species $/ 100 \mathrm{~m}^{2}$.

Native bunchgrass densities on the CCCF subunit were approximately 29 plants $/ \mathrm{m}^{2}$, composed of bluebunch wheatgrass and Sandberg's bluegrass. Native forb densities were approximately 7 plants $/ \mathrm{m}^{2}$. Exotic species cover on the CCCF subunit was higher than other subunits in Unit 4 and totaled almost $14 \%$, which was composed of primarily cheatgrass $(24 \%)$.

\subsection{Unit 5}

Unit 5 consists of the area associated with the excavated and bladed areas lying within the rock berms surrounding the observatory and sheds at the top of the ridgeline. This site was composed of large rocky substrate with minimal amounts of soil. The substrates typical of the area surrounding the heavily disturbed revegetation Unit 5 are thin rocky lithosols that support thyme buckwheat or rock buckwheat/ bunchgrass plant communities. These communities represent the desired future conditions for this area. Some contouring of the site was conducted to fill in low areas and walkway and building footprints with rocky basalt cobble from the surrounding berms. Material from the berms was also used to fill in the cisterns at the 109 debris site. Where native vegetation had already established on the basalt berms, no additional seeding or re-contouring was attempted as part of this revegetation project.

\subsubsection{Unit 5 Planting Details}

The disturbance footprint was prepared by ripping with an s-tine harrow. Bunchgrasses were broadcast seeded and harrowed to incorporate seed into the rocky substrate. Thyme buckwheat seed was then broadcast. Three seeded islands and one transplant island were created. Seeded diversity islands were broadcast seeded into circular areas with radii ranging from 0.5 to $1.4 \mathrm{~m}$ depending on the number of seeds planted. The transplant island was circular with a 4-m radius. Transplants were installed using pry bars and dibbles. Rocks were placed in a circle around each transplant, especially on the upwind side, to create a protected space for the seedlings. Seeding and transplant details are provided in Table 3.11. Seeding rates and transplant densities are provided in Appendix A. Super Tack ${ }^{\circledR}$ was applied (50-lb/ac) with supplemental watering at a nominal rate of $6000 \mathrm{gal} / \mathrm{ac}$ across the entire unit. 
Table 3.11. Seeding and Transplant Details for Unit 5

\begin{tabular}{|c|c|c|c|c|c|}
\hline Method & $\begin{array}{l}\text { Growth } \\
\text { Habit }\end{array}$ & Scientific Name & Common Name & $\begin{array}{l}\text { Whole } \\
\text { Unit }\end{array}$ & $\begin{array}{c}\text { Diversity } \\
\text { Islands }\end{array}$ \\
\hline \multirow{19}{*}{$\begin{array}{l}\text { broadcast } \\
\text { seeded }\end{array}$} & \multirow[t]{2}{*}{ bunchgrass } & Pseudoroegneria spicata & bluebunch wheatgrass & $\mathrm{x}$ & \\
\hline & & Poa secunda & Sandberg's bluegrass & $\mathrm{x}$ & \\
\hline & \multirow[t]{12}{*}{ forb } & Agastache occidentalis & western horsemint & & $\mathrm{x}$ \\
\hline & & $\begin{array}{l}\text { Astragalus conjunctus var. } \\
\text { rickardii }^{\text {(a) }}\end{array}$ & Dr. Bills locoweed & & $\mathrm{x}$ \\
\hline & & Astragalus purshii & woolly-pod milkvetch & & $\mathrm{x}$ \\
\hline & & Balsamorhiza rosea $^{(\mathrm{a})}$ & rosy balsamroot & & $\mathrm{x}$ \\
\hline & & Erigeron linearis & desert yellowdaisy & & $\mathrm{x}$ \\
\hline & & Erigeron poliospermus & cushion fleabane & & $\mathrm{x}$ \\
\hline & & $\begin{array}{l}\text { Eriophyllum lanatum var. } \\
\text { integrifolum }\end{array}$ & woolly sunflower & & $\mathrm{x}$ \\
\hline & & Lomatium grayi & Gray's desertparsley & & $\mathrm{x}$ \\
\hline & & Lomatium triternatum & nineleaf desertparsley & & $\mathrm{x}$ \\
\hline & & Penstemon speciosus & showy beardtongue & & $\mathrm{x}$ \\
\hline & & Phemeranthus spinescens & spiny flameflower & & $\mathrm{x}$ \\
\hline & & Sedum leibergii & Leiberg's stonecrop & & $\mathrm{x}$ \\
\hline & \multirow[t]{2}{*}{ shrub } & Salvia dorrii & grayball sage & & $\mathrm{x}$ \\
\hline & & Tetradymia canescens & gray horsebrush & & $\mathrm{x}$ \\
\hline & \multirow[t]{3}{*}{ subshrub } & Eriogonum sphaerocephalum & rock buckwheat & & $\mathrm{x}$ \\
\hline & & Eriogonum thymoides & thymeleaf buckwheat & $\mathrm{x}$ & \\
\hline & & Nestotus stenophyllus & narrowleaf goldenweed & & $\mathrm{x}$ \\
\hline \multirow[t]{14}{*}{ transplant } & \multirow[t]{10}{*}{ forb } & Agastache occidentalis & western horsemint & & $\mathrm{x}$ \\
\hline & & Astragalus purshii & woolly-pod milkvetch & & $\mathrm{x}$ \\
\hline & & Balsamorhiza rosea $^{(\mathrm{a})}$ & rosy balsamroot & & $\mathrm{x}$ \\
\hline & & Crepis modocensis & low hawksbeard & & $\mathrm{x}$ \\
\hline & & Erigeron linearis & desert yellowdaisy & & $\mathrm{x}$ \\
\hline & & Erigeron poliospermus & cushion fleabane & & $\mathrm{x}$ \\
\hline & & Penstemon speciosus & showy beardtongue & & $\mathrm{x}$ \\
\hline & & Phemeranthus spinescens & spiny flameflower & & $\mathrm{x}$ \\
\hline & & Phlox hoodii & Hood's phlox & & $\mathrm{x}$ \\
\hline & & Sedum leibergii & Leiberg's stonecrop & & $\mathrm{x}$ \\
\hline & \multirow{4}{*}{$\begin{array}{l}\text { shrub } \\
\text { subshrub }\end{array}$} & Salvia dorrii & grayball sage & & $\mathrm{x}$ \\
\hline & & Eriogonum sphaerocephalum & rock buckwheat & & $\mathrm{x}$ \\
\hline & & Eriogonum thymoides & thymeleaf buckwheat & & $\mathrm{x}$ \\
\hline & & Nestotus stenophyllus & narrowleaf goldenweed & & $\mathrm{x}$ \\
\hline
\end{tabular}

(a) State Watch List species.

\subsubsection{Unit 5 Vegetation Establishment}

This unit presented the greatest technical challenges to revegetation because of the rocky nature of the substrate and relative lack of fine soil material. However, native bunchgrass densities on Unit 5 were 
about 45 plants $/ \mathrm{m}^{2}$ with Sandberg's bluegrass being the primary contributor ( $\sim 40$ plants $\left./ \mathrm{m}^{2}\right)$. Substrates on Unit 5 were extremely rocky and the only native forb that grew within the $100-\mathrm{m}^{2}$ plots was white scorpionweed, which occurred at a density of slightly less than $1 \mathrm{plant} / \mathrm{m}^{2}$. Cover of exotic species was minimal $(<1 \%)$. Species richness was low on this unit, but will likely increase over time.

\subsection{Unit 6}

Revegetation at Unit 6 consists of three small areas along Rattlesnake ridge associated with decommissioning and removal of the 6635 Crown Castle facility $(0.1 \mathrm{ac})$, and the 6636 and 6637 communication towers and access road $(0.6 \mathrm{ac})$. The desired future conditions for revegetation on Unit 6 are recovery to a thyme buckwheat/bluebunch wheatgrass plant community typical of the lithosolic soils of the ridgetop. Portions of the access roads and of the facility footprint at each individual location harbored remnant native grasses and forbs.

\subsubsection{Unit 6 Planting Details}

The disturbance footprints and road tracks were prepared by ripping with an s-tine harrow. Bunchgrasses were broadcast seeded and harrowed to incorporate seed. Thyme buckwheat seed was broadcast. Five seeded islands ( 3 at 6635 Crown Castle; 2 at 6636 communication towers) and three transplant islands ( 2 at 6635 Crown Castle; 1 at 6636 communication towers) were created. The same suite of species was used for each area (Table 3.12). Seeded islands were broadcast seeded into circular areas with radii ranging from 0.5 to $1.4 \mathrm{~m}$ depending on the number of seeds planted. Transplant islands were circular with a 4-m radius except where circumstances required planting in other irregular patterns. Transplants were installed using pry bars and dibbles. As before, rocks were placed in a circle around each transplant to create a protected space for the seedlings. Super Tack ${ }^{\circledR}$ was applied $(50-\mathrm{lb} / \mathrm{ac})$ with supplemental watering at a nominal rate of $6000 \mathrm{gal} / \mathrm{ac}$ across the entire unit. Seeding rates and transplant densities are provided in Appendix A.

\subsubsection{Unit 6 Vegetation Establishment}

Native bunchgrass establishment on Unit 6 was dominated by bluebunch wheatgrass, which had densities of approximately 40 plants $/ \mathrm{m}^{2}$. Total native bunchgrass density was approximately

47 plants $/ \mathrm{m}^{2}$. Exotic cover was approximately $40 \%$ and consisted mainly of two species: doorweed (Polygonum aviculare) and Jim Hill mustard. These values exceeded initial target criteria.

\subsection{Unit 7}

Revegetation Unit 7 is located on the north-facing slope of Rattlesnake Ridge at an elevation of approximately $3000 \mathrm{ft}$. Soil at the site is a stony silt loam and the general area surrounding the site supports vegetation typical of seeps and springs occurring on hills within the shrub-steppe areas of the Columbia Plateau ecoregion, which can be characterized as Eastside Canyon Shrublands. The revegetation unit was relatively small compared to most of the other units and encompassed the area associated with the pumphouse at the springs and a small parking/turn-around area. The surrounding area also contained a number of exotic weedy species such as cheatgrass and Jim Hill mustard. 
Table 3.12. Seeding and Transplant Detail for Unit 6

\begin{tabular}{|c|c|c|c|c|c|}
\hline Method & $\begin{array}{l}\text { Growth } \\
\text { Habit }\end{array}$ & Scientific Name & Common Name & Subunit & $\begin{array}{l}\text { Diversity } \\
\text { Islands }\end{array}$ \\
\hline \multirow{14}{*}{$\begin{array}{l}\text { broadcast } \\
\text { seeded }\end{array}$} & \multirow[t]{3}{*}{ bunchgrass } & Festuca idahoensis & Idaho fescue & $\mathrm{x}$ & \\
\hline & & Pseudoroegneria spicata & bluebunch wheatgrass & $\mathrm{x}$ & \\
\hline & & Poa secunda & Sandberg's bluegrass & $\mathrm{x}$ & \\
\hline & \multirow[t]{7}{*}{ forb } & Agastache occidentalis & western horsemint & & $\mathrm{x}$ \\
\hline & & Astragalus purshii & woolly-pod milkvetch & & $\mathrm{x}$ \\
\hline & & Erigeron linearis & desert yellowdaisy & & $\mathrm{x}$ \\
\hline & & Erigeron poliospermus & cushion fleabane & & $\mathrm{x}$ \\
\hline & & $\begin{array}{l}\text { Eriophyllum lanatum var. } \\
\text { integrifolum }\end{array}$ & woolly sunflower & & $\mathrm{x}$ \\
\hline & & Lomatium grayi & Gray’s desertparsley & & $\mathrm{x}$ \\
\hline & & Sedum leibergii & Leiberg's stonecrop & & $\mathrm{x}$ \\
\hline & \multirow{4}{*}{$\begin{array}{l}\text { shrub } \\
\text { subshrub }\end{array}$} & Tetradymia canescens & gray horsebrush & & \\
\hline & & Eriogonum sphaerocephalum & rock buckwheat & & $\mathrm{x}$ \\
\hline & & Eriogonum thymoides & thymeleaf buckwheat & $\mathrm{x}$ & \\
\hline & & Nestotus stenophyllus & narrowleaf goldenweed & & $\mathrm{x}$ \\
\hline \multirow[t]{13}{*}{ transplant } & \multirow[t]{9}{*}{ forb } & Agastache occidentalis & western horsemint & & $\mathrm{x}$ \\
\hline & & Astragalus purshii & woolly-pod milkvetch & & $\mathrm{x}$ \\
\hline & & Balsamorhiza rosea ${ }^{(a)}$ & rosy balsamroot & & $\mathrm{x}$ \\
\hline & & Erigeron linearis & desert yellowdaisy & & $\mathrm{x}$ \\
\hline & & Erigeron poliospermus & cushion fleabane & & $\mathrm{x}$ \\
\hline & & Penstemon speciosus & showy beardtongue & & $\mathrm{x}$ \\
\hline & & Phemeranthus spinescens & spiny flameflower & & $\mathrm{x}$ \\
\hline & & Phlox hoodii & Hood's phlox & & $\mathrm{x}$ \\
\hline & & Sedum leibergii & Leiberg's stonecrop & & $\mathrm{x}$ \\
\hline & shrub & Salvia dorrii & grayball sage & & $\mathrm{x}$ \\
\hline & \multirow[t]{3}{*}{ subshrub } & Eriogonum sphaerocephalum & rock buckwheat & & $\mathrm{x}$ \\
\hline & & Eriogonum thymoides & thymeleaf buckwheat & & $\mathrm{x}$ \\
\hline & & Nestotus stenophyllus & narrowleaf goldenweed & & $\mathrm{x}$ \\
\hline
\end{tabular}

(a) State Watch List species.

\subsubsection{Unit 7 Planting Details}

The site was accessed with light vehicles. The footprint was prepared by hand raking. Bunchgrasses were hand broadcast and the site was raked again to incorporate seed into the stony soil. Forbs were then broadcast and a layer of Idaho fescue native straw was applied. The straw was not crimped.

Supplemental water was not applied. No seeded islands were created but transplants were installed at three locations. Forb transplants were installed in one marked irregular plot. Vine transplants were installed in two locations and marked with flagging. Transplants were installed using dibbles and hand tools. Seeding and transplant details are provided in Table 3.13. Seeding rates and transplant densities are provided in Appendix A. 
Table 3.13. Seeding and Transplant Detail for Unit 7

\begin{tabular}{|c|c|c|c|c|c|}
\hline Method & $\begin{array}{c}\text { Growth } \\
\text { Habit }\end{array}$ & Scientific Name & Common Name & $\begin{array}{l}\text { Whole } \\
\text { Unit }\end{array}$ & $\begin{array}{c}\text { Diversity } \\
\text { Islands }\end{array}$ \\
\hline \multirow{4}{*}{$\begin{array}{l}\text { broadcast } \\
\text { seeded }\end{array}$} & bunchgrass & Leymus cinereus & giant wildrye & $\mathrm{x}$ & \\
\hline & & Pseudoroegneria spicata & bluebunch wheatgrass & $\mathrm{x}$ & \\
\hline & & Poa secunda & Sandberg's bluegrass & $\mathrm{x}$ & \\
\hline & forb & Lomatium macrocarpum & bigseed desertparsley & $\mathrm{x}$ & \\
\hline \multirow[t]{3}{*}{ transplant } & forb & Agastache occidentalis & western horsemint & & $\mathrm{x}$ \\
\hline & & Astragalus purshii & woolly-pod milkvetch & & $\mathrm{x}$ \\
\hline & vine & Clematis ligusticifolia & western white clematis & & $\mathrm{x}$ \\
\hline
\end{tabular}

\subsubsection{Unit 7 Vegetation Establishment}

Native plant densities on Unit 7 were relatively low at approximately 5 to 6 plants $/ \mathrm{m}^{2}$. The primary native bunchgrass that established was bluebunch wheatgrass. Native forbs were also found in the revegetation area at a density of approximately 5 plants $/ \mathrm{m}^{2}$. Exotic cover at this site was quite high at about $48 \%$. 


\subsection{Summary and Recommendations}

Measurements of the initial vegetation establishment on the seven revegetation units on the Fitzner/Eberhard Arid Lands Ecology Reserve indicate that native plants were well established on most of the units. In most cases, the density of native bunchgrasses and forbs exceeded the initial target criteria and initial survival of transplanted and seeded forbs and shrubs was relatively high. However, several areas had fairly high canopy cover of exotic species including the Hodges Well subunit in Unit 2, Unit 6 and Unit 7. Where exotic species cover is composed primarily of summer annuals such as Jim Hill mustard and Russian thistle, the trajectory of succession is expected to favor future increases in native species cover over continued increases in alien annual species if native bunchgrasses and forbs appear to be relatively well-established at the site. This may be the case at the Hodges Well subunit.

Where cheatgrass is a primary component of the exotic species cover, additional monitoring is critical to assess whether further treatment (e.g., herbicides, weeding, or seeding) is needed to restore native vegetation. Unit 7 has a very small footprint and the surrounding area harbors weedy alien species that may affect the development of a native-dominated community on the site. However, the conditions at Unit 7 are relatively mesic and may favor native grasses and forbs over cheatgrass over time.

Unit 6 footprints are also relatively small, and the surrounding areas do not harbor a significant amount of weedy alien species. The main species contributing to exotic species cover along the roadway and where small communications facilities were removed was doorweed, an annual forb. It is unknown whether this species will persist under the climatic conditions found at the top of Rattlesnake Ridge. If this species persists or increases over the next several years, additional treatments may be needed to reduce doorweed and encourage regrowth of native species. 


\subsection{References}

DOE - U.S. Department of Energy. 2009. Environmental Assessment: Combined Community Communications Facility and Infrastructure Cleanup on the Fitzner/Eberhardt Arid Lands Ecology Reserve, Hanford Site, Richland, Washington. DOE/EA-1660F, U.S. Department of Energy, Richland, Washington.

Downs JL, RE Durham, and K.B.Larson. 2011. Revegetation Plan for Areas of the Fitzner-Eberhardt Arid Lands Ecology Reserve Affected by Decommissioning of Buildings and Infrastructure, and Debris Clean-up Actions. PNNL-19879, Pacific Northwest National Laboratory, Richland, Washington.

Hajek BF. 1966. Soil Survey, Hanford Project in Benton County, Washington. BNWL-243, Pacific Northwest Laboratory, Richland, Washington.

SAS Institute. 2002. JMP Statistics and Graphics Guide, Version 5. Cary, SAS Institute Inc.

Stohlgren TJ, MB Falkner, and LD Schell. 1995. “A Modified-Whittaker Nested Vegetation Sampling Method." Vegetatio 117:113-121.

Stone WA, JM Thorp, OP Gifford, and DJ Hoitink. 1983. Climatological Summary for the Hanford Area. PNL-4622, Pacific Northwest Laboratory, Richland, Washington. 
Appendix A

\section{Planting Details}


Table A.1. Unit Seeding Rates (lb/ac) and Methods Used

\begin{tabular}{|c|c|c|c|c|c|}
\hline Unit & Subunit & Method & $\begin{array}{c}\text { Growth } \\
\text { Habit }\end{array}$ & Species & $\begin{array}{l}\text { Application } \\
\text { Rate (lb/ac) }\end{array}$ \\
\hline \multirow[t]{7}{*}{ Unit 1} & Rattlesnake Springs & drill seeded & bunchgrass & Leymus cinereus & 20.0 \\
\hline & & & & Poa secunda & 20.0 \\
\hline & & broadcast seeded & forb & Achillea millefolium & 1.4 \\
\hline & & & & Balsamorhiza careyana & 3.7 \\
\hline & & & & Sphaeralcea munroana & (a) \\
\hline & & & & Lomatium macrocarpum & 0.3 \\
\hline & & & shrub & Artemisia tridentata & 2.8 \\
\hline \multirow[t]{30}{*}{ Unit 2} & ALE Ecology buildings & drill seeded & bunchgrass & Pseudoroegneria spicata & 20.0 \\
\hline & & & & Poa secunda & 20.0 \\
\hline & & broadcast seeded & bunchgrass & Elymus elymoides & (a) \\
\hline & & & forb & Achillea millefolium & 1.3 \\
\hline & & & & Balsamorhiza careyana & 3.7 \\
\hline & & & & Machaeranthera canescens & (a) \\
\hline & & & & Crepis atribarba & (a) \\
\hline & & & shrub & Artemisia tridentata & 2.7 \\
\hline & Hodges Well (HW) & drill seeded & bunchgrass & Pseudoroegneria spicata & 20.0 \\
\hline & & & & Poa secunda & 20.0 \\
\hline & & broadcast seeded & bunchgrass & Achnatherum hymenoides & (a) \\
\hline & & & & Elymus elymoides & (a) \\
\hline & & & forb & Achillea millefolium & 1.4 \\
\hline & & & & Balsamorhiza careyana & 3.7 \\
\hline & & & & Crepis atribarba & (a) \\
\hline & & & & Lomatium macrocarpum & 0.3 \\
\hline & & & & Machaeranthera canescens & (a) \\
\hline & & & shrub & Artemisia tridentata & 2.8 \\
\hline & Debris site 50 & broadcast seeded & bunchgrass & Pseudoroegneria spicata & 12.5 \\
\hline & & & & Poa secunda & 12.5 \\
\hline & & & forb & Achillea millefolium & 0.4 \\
\hline & & & & Balsamorhiza careyana & 1.0 \\
\hline & & & & Lomatium macrocarpum & 0.3 \\
\hline & & & shrub & Artemisia tridentata & 1.7 \\
\hline & Debris site 60 & broadcast seeded & bunchgrass & Pseudoroegneria spicata & 12.4 \\
\hline & & & & Poa secunda & 12.4 \\
\hline & & & forb & Achillea millefolium & 1.4 \\
\hline & & & & Balsamorhiza careyana & 3.7 \\
\hline & & & & Lomatium macrocarpum & 0.3 \\
\hline & & & shrub & Artemisia tridentata & 16.8 \\
\hline
\end{tabular}


Table A.1. (contd)

\begin{tabular}{|c|c|c|c|c|c|}
\hline Unit & Subunit & Method & $\begin{array}{l}\text { Growth } \\
\text { Habit }\end{array}$ & Species & $\begin{array}{l}\text { Application } \\
\text { Rate (lb/ac) }\end{array}$ \\
\hline & \multirow{6}{*}{$\begin{array}{l}\text { Debris site } 109 \text { (gas } \\
\text { wells) }\end{array}$} & \multirow[t]{6}{*}{ broadcast seeded } & \multirow[t]{2}{*}{ bunchgrass } & Pseudoroegneria spicata & 11.1 \\
\hline & & & & Poa secunda & 11.1 \\
\hline & & & \multirow[t]{3}{*}{ forb } & Achillea millefolium & 1.2 \\
\hline & & & & Balsamorhiza careyana & 3.2 \\
\hline & & & & Lomatium macrocarpum & 0.2 \\
\hline & & & shrub & Artemisia tridentata & 2.5 \\
\hline & \multirow[t]{6}{*}{ Debris site 127} & \multirow[t]{6}{*}{ broadcast seeded } & \multirow[t]{2}{*}{ bunchgrass } & Pseudoroegneria spicata & 12.5 \\
\hline & & & & Poa secunda & 12.5 \\
\hline & & & \multirow[t]{3}{*}{ forb } & Achillea millefolium & 1.3 \\
\hline & & & & Balsamorhiza careyana & 3.4 \\
\hline & & & & Lomatium macrocarpum & 0.3 \\
\hline & & & shrub & Artemisia tridentata & 25.9 \\
\hline & \multirow{6}{*}{$\begin{array}{l}\text { Debris sites } 138,139 \\
\text { and } 146\end{array}$} & \multirow[t]{6}{*}{ broadcast seeded } & \multirow[t]{2}{*}{ bunchgrass } & Pseudoroegneria spicata & 12.5 \\
\hline & & & & Poa secunda & 12.5 \\
\hline & & & \multirow[t]{3}{*}{ forb } & Achillea millefolium & 1.7 \\
\hline & & & & Balsamorhiza careyana & 4.6 \\
\hline & & & & Lomatium macrocarpum & 0.3 \\
\hline & & & shrub & Artemisia tridentata & 2.0 \\
\hline \multirow[t]{4}{*}{ Unit 3} & \multirow[t]{4}{*}{ Radio-telescope } & \multirow[t]{4}{*}{ broadcast seeded } & \multirow[t]{3}{*}{ bunchgrass } & Festuca idahoensis & 9.6 \\
\hline & & & & Pseudoroegneria spicata & 15.2 \\
\hline & & & & Poa secunda & 15.2 \\
\hline & & & subshrub & Eriogonum thymoides & 0.3 \\
\hline \multirow[t]{4}{*}{ Unit 4} & \multirow[t]{4}{*}{ All sub units } & \multirow[t]{4}{*}{ broadcast seeded } & \multirow[t]{3}{*}{ bunchgrass } & Festuca idahoensis & 9.6 \\
\hline & & & & Pseudoroegneria spicata & 15.2 \\
\hline & & & & Poa secunda & 15.2 \\
\hline & & & subshrub & Eriogonum thymoides & 0.1 \\
\hline \multirow[t]{3}{*}{ Unit 5} & \multirow{3}{*}{$\begin{array}{c}\text { 6652-C Shed and Berm } \\
\text { Area }\end{array}$} & \multirow[t]{3}{*}{ broadcast seeded } & \multirow[t]{2}{*}{ bunchgrass } & Pseudoroegneria spicata & 20.0 \\
\hline & & & & Poa secunda & 20.0 \\
\hline & & & subshrub & Eriogonum thymoides & 0.1 \\
\hline \multirow[t]{4}{*}{ Unit 6} & \multirow[t]{4}{*}{ All sub units } & \multirow[t]{4}{*}{ broadcast seeded } & bunchgrass & Festuca idahoensis & 9.6 \\
\hline & & & & Pseudoroegneria spicata & 15.2 \\
\hline & & & & Poa secunda & 15.2 \\
\hline & & & subshrub & Eriogonum thymoides & 0.1 \\
\hline Unit 7 & 6652-T Pumphouse & broadcast seeded & bunchgrass & Leymus cinereus & 8.1 \\
\hline & & & & Pseudoroegneria spicata & 16.2 \\
\hline & & & & Poa secunda & 16.2 \\
\hline & & & forb & Lomatium macrocarpum & 0.028 \\
\hline
\end{tabular}

(a) Unknown quantity of seed scattered around the unit from on-hand local collections. 
Table A.2. Wyoming Big Sagebrush Transplant Detail by Unit, Number Planted, Area, and Rate (plants/ac)

\begin{tabular}{llccc}
\hline Unit & Subunit & $\begin{array}{c}\text { Number } \\
\text { Planted }\end{array}$ & $\begin{array}{c}\text { Area }^{(\mathrm{a})} \\
(\mathrm{ac})\end{array}$ & $\begin{array}{c}\text { Rate } \\
\text { (plants/ac) }\end{array}$ \\
\hline Unit 1 & Rattlesnake Springs & 240 & 0.5 & 480 \\
Unit 2 & ALE HQ & 1260 & 2 & 630 \\
& Hodges Well & 400 & 0.5 & 800 \\
& Debris site 40 & 10 & 0.01 & 1000 \\
& Debris site 50 & 20 & 0.036 & 556 \\
& Debris site 60 & 30 & 0.025 & 1200 \\
& Debris site 109 (Roadway) & 184 & 1.086 & 169 \\
& Debris site 138 (Cistern) & 15 & 0.006 & 2500 \\
& Debris site 139 (Cistern) & 30 & 0.04 & 750 \\
& Debris site 146 & 45 & 0.07 & 643 \\
Totals & & $\mathbf{2 2 3 4}$ & $\mathbf{4 . 2 7 3}$ & $\mathbf{5 2 3}$ \\
\hline
\end{tabular}

(a) Area transplanted.

(b) Average number of plants per acre. 
Table A.3. Transplanted Diversity Islands: Transplants by Unit, Sub Unit, Island Plot ID, and Species ${ }^{(a)}$

\begin{tabular}{|c|c|c|c|c|c|}
\hline $\begin{array}{c}\text { Revegetation } \\
\text { Unit }\end{array}$ & Subunit & $\begin{array}{l}\text { Island Plot } \\
\text { ID Number }\end{array}$ & Growth Habit & Scientific Name & $\begin{array}{c}\text { Number of } \\
\text { Transplants } \\
\text { per Island }\end{array}$ \\
\hline \multirow[t]{6}{*}{ Unit 1} & \multirow{6}{*}{$\begin{array}{l}\text { Rattlesnake } \\
\text { Springs }\end{array}$} & Circle & forb & Agoseris grandiflora & 12 \\
\hline & & & & Astragalus caricinus & 12 \\
\hline & & & shrub & Grayia spinosa & 150 \\
\hline & & Road & forb & Agoseris grandiflora & 13 \\
\hline & & & & Astragalus caricinus & 13 \\
\hline & & & shrub & Grayia spinosa & 150 \\
\hline \multirow[t]{6}{*}{ Unit 2} & \multirow[t]{6}{*}{ ALE HQ } & \multirow{6}{*}{$\begin{array}{c}280,281 \\
282,283, \\
1697 \text { and } \\
1698\end{array}$} & \multirow{6}{*}{$\begin{array}{l}\text { bunchgrass } \\
\text { forb }\end{array}$} & Achnatherum thurberianum & 7 \\
\hline & & & & Agoseris grandiflora & 21 \\
\hline & & & & Astragalus caricinus & 25 \\
\hline & & & & Erigeron piperianus & 39 \\
\hline & & & & Lupinus sericeus & 6 \\
\hline & & & & Phlox longifolia & 16 \\
\hline \multirow[t]{13}{*}{ Unit 3} & \multirow[t]{13}{*}{ Radio-telescope } & \multirow[t]{13}{*}{65} & \multirow[t]{9}{*}{ forb } & Agastache occidentalis & 24 \\
\hline & & & & Astragalus purshii & 35 \\
\hline & & & & Balsamorhiza rosea & 2 \\
\hline & & & & Erigeron linearis & 43 \\
\hline & & & & Erigeron poliospermus & 3 \\
\hline & & & & Penstemon speciosus & 1 \\
\hline & & & & Phemeranthus spinescens & 15 \\
\hline & & & & Phlox hoodii & 1 \\
\hline & & & & Sedum leibergii & 1 \\
\hline & & & \multirow{4}{*}{$\begin{array}{c}\text { shrub } \\
\text { subshrub }\end{array}$} & Salvia dorrii & 5 \\
\hline & & & & Eriogonum sphaerocephalum & 4 \\
\hline & & & & Eriogonum thymoides & 3 \\
\hline & & & & Nestotus stenophyllus & 1 \\
\hline \multirow[t]{13}{*}{ Unit 4} & \multirow{13}{*}{$\begin{array}{c}\text { 6652-C } \\
\text { Buildings }\end{array}$} & \multirow[t]{13}{*}{64} & \multirow[t]{9}{*}{ forb } & Agastache occidentalis & 24 \\
\hline & & & & Astragalus purshii & 35 \\
\hline & & & & Balsamorhiza rosea & 2 \\
\hline & & & & Erigeron linearis & 43 \\
\hline & & & & Erigeron poliospermus & 3 \\
\hline & & & & Penstemon speciosus & 1 \\
\hline & & & & Phemeranthus spinescens & 15 \\
\hline & & & & Phlox hoodii & 1 \\
\hline & & & & Sedum leibergii & 1 \\
\hline & & & \multirow{4}{*}{$\begin{array}{c}\text { shrub } \\
\text { subshrub }\end{array}$} & Salvia dorrii & 5 \\
\hline & & & & Eriogonum sphaerocephalum & 4 \\
\hline & & & & Eriogonum thymoides & 3 \\
\hline & & & & Nestotus stenophyllus & 1 \\
\hline
\end{tabular}


Table A.3. (contd)

\begin{tabular}{|c|c|c|c|c|c|}
\hline $\begin{array}{l}\text { Revegetation } \\
\text { Unit }\end{array}$ & Subunit & $\begin{array}{l}\text { Island Plot } \\
\text { ID Number }\end{array}$ & Growth Habit & Scientific Name & $\begin{array}{c}\text { Number of } \\
\text { Transplants } \\
\text { per Island }\end{array}$ \\
\hline & & \multirow[t]{14}{*}{284} & \multirow[t]{10}{*}{ forb } & Agastache occidentalis & 24 \\
\hline & & & & Astragalus purshii & 29 \\
\hline & & & & Balsamorhiza rosea & 2 \\
\hline & & & & Crepis modocensis & 1 \\
\hline & & & & Erigeron linearis & 43 \\
\hline & & & & Erigeron poliospermus & 3 \\
\hline & & & & Penstemon speciosus & 1 \\
\hline & & & & Phemeranthus spinescens & 15 \\
\hline & & & & Phlox hoodii & 1 \\
\hline & & & & Sedum leibergii & 4 \\
\hline & & & shrub & Salvia dorrii & 5 \\
\hline & & & \multirow[t]{3}{*}{ subshrub } & Eriogonum sphaerocephalum & 3 \\
\hline & & & & Eriogonum thymoides & 2 \\
\hline & & & & Nestotus stenophyllus & 1 \\
\hline & & \multirow[t]{14}{*}{285} & \multirow[t]{10}{*}{ forb } & Agastache occidentalis & 24 \\
\hline & & & & Astragalus purshii & 29 \\
\hline & & & & Balsamorhiza rosea & 2 \\
\hline & & & & Crepis modocensis & 1 \\
\hline & & & & Erigeron linearis & 43 \\
\hline & & & & Erigeron poliospermus & 3 \\
\hline & & & & Penstemon speciosus & 1 \\
\hline & & & & Phemeranthus spinescens & 15 \\
\hline & & & & Phlox hoodii & 1 \\
\hline & & & & Sedum leibergii & 1 \\
\hline & & & \multirow{4}{*}{$\begin{array}{c}\text { shrub } \\
\text { subshrub }\end{array}$} & Salvia dorrii & 5 \\
\hline & & & & Eriogonum sphaerocephalum & 3 \\
\hline & & & & Eriogonum thymoides & 3 \\
\hline & & & & Nestotus stenophyllus & 1 \\
\hline & & \multirow[t]{12}{*}{286} & \multirow[t]{10}{*}{ forb } & Agastache occidentalis & 24 \\
\hline & & & & Astragalus purshii & 35 \\
\hline & & & & Balsamorhiza rosea & 2 \\
\hline & & & & Crepis modocensis & 1 \\
\hline & & & & Erigeron linearis & 43 \\
\hline & & & & Erigeron poliospermus & 3 \\
\hline & & & & Penstemon speciosus & 1 \\
\hline & & & & Phemeranthus spinescens & 15 \\
\hline & & & & Phlox hoodii & 1 \\
\hline & & & & Sedum leibergii & 1 \\
\hline & & & \multirow{2}{*}{$\begin{array}{c}\text { shrub } \\
\text { subshrub }\end{array}$} & Salvia dorrii & 5 \\
\hline & & & & Eriogonum sphaerocephalum & 3 \\
\hline
\end{tabular}


Table A.3. (contd)

\begin{tabular}{|c|c|c|c|c|c|}
\hline $\begin{array}{c}\text { Revegetation } \\
\text { Unit }\end{array}$ & Subunit & $\begin{array}{l}\text { Island Plot } \\
\text { ID Number }\end{array}$ & Growth Habit & Scientific Name & $\begin{array}{c}\text { Number of } \\
\text { Transplants } \\
\text { per Island }\end{array}$ \\
\hline & & & & Eriogonum thymoides & 3 \\
\hline & & & & Nestotus stenophyllus & 1 \\
\hline & & 287 & forb & Agastache occidentalis & 24 \\
\hline & & & & Astragalus purshii & 35 \\
\hline & & & & Balsamorhiza rosea & 2 \\
\hline & & & & Crepis modocensis & 1 \\
\hline & & & & Erigeron linearis & 43 \\
\hline & & & & Erigeron poliospermus & 3 \\
\hline & & & & Penstemon speciosus & 1 \\
\hline & & & & Phemeranthus spinescens & 15 \\
\hline & & & & Phlox hoodii & 1 \\
\hline & & & & Sedum leibergii & 1 \\
\hline & & & shrub & Salvia dorrii & 5 \\
\hline & & & subshrub & Eriogonum sphaerocephalum & 3 \\
\hline & & & & Eriogonum thymoides & 3 \\
\hline & & & & Nestotus stenophyllus & 1 \\
\hline & & 1592 & forb & Astragalus purshii & 12 \\
\hline & & & shrub & Salvia dorrii & 3 \\
\hline \multirow[t]{14}{*}{ Unit 5} & \multirow{14}{*}{$\begin{array}{l}\text { 6652-C Shed } \\
\text { and Berm }\end{array}$} & 1798 & forb & Agastache occidentalis & 24 \\
\hline & & & & Astragalus purshii & 33 \\
\hline & & & & Balsamorhiza rosea & 2 \\
\hline & & & & Crepis modocensis & 1 \\
\hline & & & & Erigeron linearis & 43 \\
\hline & & & & Erigeron poliospermus & 3 \\
\hline & & & & Penstemon speciosus & 1 \\
\hline & & & & Phemeranthus spinescens & 15 \\
\hline & & & & Phlox hoodii & 1 \\
\hline & & & & Sedum leibergii & 4 \\
\hline & & & shrub & Salvia dorrii & 5 \\
\hline & & & subshrub & Eriogonum sphaerocephalum & 3 \\
\hline & & & & Eriogonum thymoides & 2 \\
\hline & & & & Nestotus stenophyllus & 1 \\
\hline \multirow[t]{8}{*}{ Unit 6} & \multirow{8}{*}{$\begin{array}{l}6635 \text { Crown } \\
\text { Castle }\end{array}$} & \multirow[t]{8}{*}{66} & \multirow[t]{8}{*}{ forb } & Agastache occidentalis & 24 \\
\hline & & & & Astragalus purshii & 35 \\
\hline & & & & Balsamorhiza rosea & 1 \\
\hline & & & & Erigeron linearis & 43 \\
\hline & & & & Erigeron poliospermus & 3 \\
\hline & & & & Penstemon speciosus & 1 \\
\hline & & & & Phemeranthus spinescens & 15 \\
\hline & & & & Phlox hoodii & 1 \\
\hline
\end{tabular}


Table A.3. (contd)

\begin{tabular}{|c|c|c|c|c|c|}
\hline $\begin{array}{l}\text { Revegetation } \\
\text { Unit }\end{array}$ & Subunit & $\begin{array}{l}\text { Island Plot } \\
\text { ID Number }\end{array}$ & Growth Habit & Scientific Name & $\begin{array}{c}\text { Number of } \\
\text { Transplants } \\
\text { per Island }\end{array}$ \\
\hline & & & & Sedum leibergii & 1 \\
\hline & & & shrub & Salvia dorrii & 3 \\
\hline & & & subshrub & Eriogonum sphaerocephalum & 4 \\
\hline & & & & Eriogonum thymoides & 2 \\
\hline & & & & Nestotus stenophyllus & 1 \\
\hline & & $1590^{(\mathrm{b})}$ & forb & Agastache occidentalis & 24 \\
\hline & & & & Astragalus purshii & 35 \\
\hline & & & & Balsamorhiza rosea & 1 \\
\hline & & & & Erigeron linearis & 44 \\
\hline & & & & Erigeron poliospermus & 3 \\
\hline & & & & Penstemon speciosus & 1 \\
\hline & & & & Phemeranthus spinescens & 15 \\
\hline & & & & Phlox hoodii & 2 \\
\hline & & & & Sedum leibergii & 1 \\
\hline & & & shrub & Salvia dorrii & 5 \\
\hline & & & subshrub & Eriogonum sphaerocephalum & 4 \\
\hline & & & & Eriogonum thymoides & 2 \\
\hline & & & & Nestotus stenophyllus & 1 \\
\hline & & 1591 & forb & Agastache occidentalis & 24 \\
\hline & Communication & & & Astragalus purshii & 37 \\
\hline & & & & Balsamorhiza rosea & 1 \\
\hline & & & & Erigeron linearis & 43 \\
\hline & & & & Erigeron poliospermus & 6 \\
\hline & & & & Penstemon speciosus & 2 \\
\hline & & & & Phemeranthus spinescens & 8 \\
\hline & & & & Phlox hoodii & 3 \\
\hline & & & shrub & Salvia dorrii & 5 \\
\hline & & & subshrub & Eriogonum sphaerocephalum & 6 \\
\hline & & & & Eriogonum thymoides & 2 \\
\hline & & & & Nestotus stenophyllus & 1 \\
\hline \multirow[t]{3}{*}{ Unit 7} & $\begin{array}{c}6652-\mathrm{T} \\
\text { Pumphouse }\end{array}$ & $\begin{array}{l}\text { flag near } \\
\text { shrubs }\end{array}$ & vine & Clematis ligusticifolia & 6 \\
\hline & & pin nw edge & forb & Agastache occidentalis & 32 \\
\hline & & pin nw edge & forb & Astragalus purshii & 12 \\
\hline \multicolumn{6}{|c|}{$\begin{array}{l}\text { Except where noted, all islands were circular in shape. Unit } 2 \text { islands had a 5-m radius; islands in Units 3, 4, 5, } \\
\text { and } 6 \text { had a 4-m radius; islands in Units } 1 \text { and } 7 \text { were irregularly shaped as noted. } \\
\text { Rectangular-shaped island. }\end{array}$} \\
\hline
\end{tabular}


Table A.4. Seeded Diversity Islands: Species by Unit, Sub Unit, and Island Plot ID Numbers ${ }^{(a)}$

\begin{tabular}{|c|c|c|c|c|c|}
\hline $\begin{array}{l}\text { Revegetation } \\
\text { Unit }\end{array}$ & Subunit & $\begin{array}{l}\text { Island ID } \\
\text { Numbers }\end{array}$ & $\begin{array}{l}\text { Growth } \\
\text { Habit }\end{array}$ & Scientific Name & $\begin{array}{l}\text { Average } \\
\text { Number of } \\
\text { Seeds per } \\
\text { Island }\end{array}$ \\
\hline \multirow[t]{6}{*}{1} & \multirow{6}{*}{$\begin{array}{l}\text { Rattlesnake } \\
\text { springs }\end{array}$} & \multirow{6}{*}{$\begin{array}{c}1581 \text {, } \\
1582 \text {, and } \\
1583\end{array}$} & forb & Astragalus succumbens & 1052 \\
\hline & & & & Chaenactis douglasii & 885 \\
\hline & & & & Crepis atribarba & 2961 \\
\hline & & & & Machaeranthera canescens & 3226 \\
\hline & & & shrub & Grayia spinosa & 1645 \\
\hline & & & vine & Clematis ligusticifolia & 92 \\
\hline \multirow[t]{30}{*}{2} & \multirow[t]{10}{*}{ ALE HQ } & \multirow{10}{*}{$\begin{array}{c}1568, \\
1569, \\
1570, \\
1571, \\
1572, \\
1573, \\
1574, \text { and } \\
1575\end{array}$} & forb & Astragalus succumbens & 472 \\
\hline & & & & Calochortus macrocarpus & 1204 \\
\hline & & & & Chaenactis douglasii & 238 \\
\hline & & & & Crepis atribarba & 649 \\
\hline & & & & Erigeron filifolius $^{(\mathrm{b})}$ & 688 \\
\hline & & & & Erigeron piperianus & 266 \\
\hline & & & & Helianthus cusickii & 518 \\
\hline & & & & Lupinus sericeus & 84 \\
\hline & & & & Machaeranthera canescens & 2912 \\
\hline & & & shrub & Ericameria nauseosa & 1170 \\
\hline & \multirow{10}{*}{$\begin{array}{l}\text { Debris site } 109 \\
\text { (gas wells) }\end{array}$} & \multirow[t]{10}{*}{1585} & forb & Astragalus succumbens & 594 \\
\hline & & & & Calochortus macrocarpus & 1489 \\
\hline & & & & Chaenactis douglasii & 705 \\
\hline & & & & Crepis atribarba & 691 \\
\hline & & & & Erigeron filifolius & 454 \\
\hline & & & & Erigeron piperianus & 215 \\
\hline & & & & Helianthus cusickii & 482 \\
\hline & & & & Lupinus sericeus & 83 \\
\hline & & & & Machaeranthera canescens & 3451 \\
\hline & & & shrub & Ericameria nauseosa & 977 \\
\hline & \multirow{10}{*}{$\begin{array}{l}\text { Debris site } 138 \\
\quad \text { (Cistern) }\end{array}$} & \multirow[t]{10}{*}{1584} & forb & Astragalus succumbens & 463 \\
\hline & & & & Calochortus macrocarpus & 1157 \\
\hline & & & & Chaenactis douglasii & 754 \\
\hline & & & & Crepis atribarba & 632 \\
\hline & & & & Erigeron filifolius & 528 \\
\hline & & & & Erigeron piperianus & 175 \\
\hline & & & & Helianthus cusickii & 443 \\
\hline & & & & Lupinus sericeus & 79 \\
\hline & & & & Machaeranthera canescens & 2838 \\
\hline & & & shrub & Ericameria nauseosa & 1061 \\
\hline
\end{tabular}


Table A.4. (contd)

\begin{tabular}{|c|c|c|c|c|c|}
\hline $\begin{array}{l}\text { Revegetation } \\
\text { Unit }\end{array}$ & Subunit & $\begin{array}{l}\text { Island ID } \\
\text { Numbers }\end{array}$ & $\begin{array}{c}\text { Growth } \\
\text { Habit }\end{array}$ & Scientific Name & $\begin{array}{l}\text { Average } \\
\text { Number of } \\
\text { Seeds per } \\
\text { Island }\end{array}$ \\
\hline & \multirow[t]{10}{*}{ Debris site 146} & \multirow{10}{*}{$\begin{array}{c}1579 \text { and } \\
1580\end{array}$} & \multirow[t]{9}{*}{ forb } & Astragalus succumbens & 490 \\
\hline & & & & Calochortus macrocarpus & 1365 \\
\hline & & & & Chaenactis douglasii & 760 \\
\hline & & & & Crepis atribarba & 684 \\
\hline & & & & Erigeron filifolius & 454 \\
\hline & & & & Erigeron piperianus & 160 \\
\hline & & & & Helianthus cusickii & 556 \\
\hline & & & & Lupinus sericeus & 82 \\
\hline & & & & Machaeranthera canescens & 2276 \\
\hline & & & shrub & Ericameria nauseosa & 1370 \\
\hline & \multirow[t]{10}{*}{ Debris site 50} & \multirow[t]{10}{*}{1586} & \multirow[t]{9}{*}{ forb } & Astragalus succumbens & 533 \\
\hline & & & & Calochortus macrocarpus & 1558 \\
\hline & & & & Chaenactis douglasii & 890 \\
\hline & & & & Crepis atribarba & 715 \\
\hline & & & & Erigeron filifolius & 786 \\
\hline & & & & Erigeron piperianus & 177 \\
\hline & & & & Helianthus cusickii & 435 \\
\hline & & & & Lupinus sericeus & 68 \\
\hline & & & & Machaeranthera canescens & 2610 \\
\hline & & & shrub & Ericameria nauseosa & 881 \\
\hline & \multirow[t]{11}{*}{ Debris site 60} & \multirow{11}{*}{$\begin{array}{c}1587 \\
\text { upslope } \\
\text { and } 1588 \\
\text { downslope }\end{array}$} & \multirow[t]{10}{*}{ forb } & Astragalus caricinus $^{(\mathrm{c})}$ & 200 \\
\hline & & & & Astragalus succumbens ${ }^{(\mathrm{d})}$ & 484 \\
\hline & & & & Calochortus macrocarpus & 1288 \\
\hline & & & & Chaenactis douglasii $^{(\mathrm{d})}$ & 802 \\
\hline & & & & Crepis atribarba & 824 \\
\hline & & & & Erigeron filifolius & 514 \\
\hline & & & & Erigeron piperianus & 266 \\
\hline & & & & Helianthus cusickii & 406 \\
\hline & & & & Lupinus sericeus & 82 \\
\hline & & & & Machaeranthera canescens & 2410 \\
\hline & & & shrub & Ericameria nauseosa & 861 \\
\hline & \multirow[t]{8}{*}{ Hodges Well } & \multirow{8}{*}{$\begin{array}{c}1576, \\
1577 \text {, and } \\
1578\end{array}$} & \multirow[t]{8}{*}{ forb } & Astragalus succumbens & 495 \\
\hline & & & & Calochortus macrocarpus & 1304 \\
\hline & & & & Chaenactis douglasii & 733 \\
\hline & & & & Crepis atribarba & 681 \\
\hline & & & & Erigeron filifolius & 552 \\
\hline & & & & Erigeron piperianus & 180 \\
\hline & & & & Helianthus cusickii & 544 \\
\hline & & & & Lupinus sericeus & 77 \\
\hline
\end{tabular}


Table A.4. (contd)

\begin{tabular}{|c|c|c|c|c|c|}
\hline $\begin{array}{l}\text { Revegetation } \\
\text { Unit }\end{array}$ & Subunit & $\begin{array}{l}\text { Island ID } \\
\text { Numbers }\end{array}$ & $\begin{array}{l}\text { Growth } \\
\text { Habit }\end{array}$ & Scientific Name & $\begin{array}{l}\text { Average } \\
\text { Number of } \\
\text { Seeds per } \\
\text { Island }\end{array}$ \\
\hline & & & & Machaeranthera canescens & 3284 \\
\hline & & & shrub & Ericameria nauseosa & 1108 \\
\hline \multirow[t]{6}{*}{3} & \multirow[t]{6}{*}{ Radio-telescope } & \multirow[t]{6}{*}{1589} & forb & Agastache occidentalis & 517 \\
\hline & & & & Astragalus purshii & 794 \\
\hline & & & & Lomatium macrocarpum & 400 \\
\hline & & & & Lupinus sericeus & 402 \\
\hline & & & shrub & Ericameria nauseosa & 169 \\
\hline & & & subshrub & Eriogonum sphaerocephalum & 76 \\
\hline \multirow[t]{27}{*}{4} & \multirow{15}{*}{$\begin{array}{l}\text { 6652-C Building } \\
\text { (barracks) }\end{array}$} & \multirow{15}{*}{$\begin{array}{c}259,260 \\
261,262 \\
263,264 \\
\text { and } 265\end{array}$} & \multirow[t]{11}{*}{ forb } & Agastache occidentalis & 50 \\
\hline & & & & Astragalus conjunctus var. rickardii & 50 \\
\hline & & & & Astragalus purshii & 50 \\
\hline & & & & Balsamorhiza rosea & 50 \\
\hline & & & & Erigeron linearis & 50 \\
\hline & & & & Erigeron poliospermus & 50 \\
\hline & & & & $\begin{array}{l}\text { Eriophyllum lanatum var. } \\
\text { integrifolum }\end{array}$ & 50 \\
\hline & & & & Lomatium grayi & 50 \\
\hline & & & & Penstemon speciosus & 50 \\
\hline & & & & Phemeranthus spinescens & 50 \\
\hline & & & & Sedum leibergii & 50 \\
\hline & & & \multirow[t]{2}{*}{ shrub } & Salvia dorrii & 50 \\
\hline & & & & Tetradymia canescens & 50 \\
\hline & & & \multirow[t]{2}{*}{ subshrub } & Eriogonum sphaerocephalum & 50 \\
\hline & & & & Nestotus stenophyllus & 50 \\
\hline & \multirow[t]{12}{*}{$\mathrm{CCF}$} & \multirow{12}{*}{$\begin{array}{l}269,270 \\
272,273, \\
274,275 \\
\text { and } 276\end{array}$} & \multirow[t]{9}{*}{ forb } & Agastache occidentalis & 50 \\
\hline & & & & Astragalus purshii & 50 \\
\hline & & & & Balsamorhiza rosea ${ }^{(\mathrm{e})}$ & 50 \\
\hline & & & & Erigeron linearis & 50 \\
\hline & & & & Erigeron poliospermus & 50 \\
\hline & & & & $\begin{array}{l}\text { Eriophyllum lanatum var. } \\
\text { integrifolum }\end{array}$ & 50 \\
\hline & & & & Lomatium grayi & 50 \\
\hline & & & & Lomatium triternatum $^{(\mathrm{f})}$ & 50 \\
\hline & & & & Sedum leibergii & 50 \\
\hline & & & \multirow{3}{*}{$\begin{array}{c}\text { shrub } \\
\text { subshrub }\end{array}$} & Tetradymia canescens & 50 \\
\hline & & & & Eriogonum sphaerocephalum & 50 \\
\hline & & & & Nestotus stenophyllus & 50 \\
\hline
\end{tabular}


Table A.4. (contd)

\begin{tabular}{|c|c|c|c|c|c|}
\hline $\begin{array}{l}\text { Revegetation } \\
\text { Unit }\end{array}$ & Subunit & $\begin{array}{l}\text { Island ID } \\
\text { Numbers }\end{array}$ & $\begin{array}{l}\text { Growth } \\
\text { Habit }\end{array}$ & Scientific Name & $\begin{array}{l}\text { Average } \\
\text { Number of } \\
\text { Seeds per } \\
\text { Island }\end{array}$ \\
\hline \multirow[t]{16}{*}{5} & \multirow{16}{*}{$\begin{array}{c}\text { 6652-C Shed and } \\
\text { Berm Area }\end{array}$} & \multirow{16}{*}{$\begin{array}{l}266,267 \\
\text { and } 268\end{array}$} & \multirow[t]{12}{*}{ forb } & Agastache occidentalis & 50 \\
\hline & & & & $\begin{array}{l}\text { Astragalus conjunctus var. } \\
\text { rickardii }^{\mathrm{g})}\end{array}$ & 50 \\
\hline & & & & Astragalus purshii & 50 \\
\hline & & & & Balsamorhiza rosea & 50 \\
\hline & & & & Erigeron linearis & 50 \\
\hline & & & & Erigeron poliospermus & 50 \\
\hline & & & & $\begin{array}{l}\text { Eriophyllum lanatum var. } \\
\text { integrifolum }\end{array}$ & 50 \\
\hline & & & & Lomatium grayi & 50 \\
\hline & & & & Lomatium triternatum $^{(\mathrm{h})}$ & 50 \\
\hline & & & & Penstemon speciosus ${ }^{(\mathrm{g})}$ & 50 \\
\hline & & & & Phemeranthus spinescens & 50 \\
\hline & & & & Sedum leibergii & 50 \\
\hline & & & \multirow[t]{2}{*}{ shrub } & Salvia dorrii & 50 \\
\hline & & & & Tetradymia canescens & 50 \\
\hline & & & \multirow[t]{2}{*}{ subshrub } & Eriogonum sphaerocephalum & 50 \\
\hline & & & & Nestotus stenophyllus & 50 \\
\hline \multirow[t]{10}{*}{6} & \multirow{10}{*}{$\begin{array}{l}6635 \text { Crown } \\
\text { Castle }\end{array}$} & \multirow[t]{10}{*}{277} & \multirow[t]{7}{*}{ forb } & Agastache occidentalis & 50 \\
\hline & & & & Astragalus purshii & 50 \\
\hline & & & & Erigeron linearis & 50 \\
\hline & & & & Erigeron poliospermus & 50 \\
\hline & & & & $\begin{array}{l}\text { Eriophyllum lanatum var. } \\
\text { integrifolum }\end{array}$ & 50 \\
\hline & & & & Lomatium grayi & 50 \\
\hline & & & & Sedum leibergii & 50 \\
\hline & & & \multirow{3}{*}{$\begin{array}{c}\text { shrub } \\
\text { subshrub }\end{array}$} & Tetradymia canescens & 50 \\
\hline & & & & Eriogonum sphaerocephalum & 50 \\
\hline & & & & Nestotus stenophyllus & 50 \\
\hline
\end{tabular}


Table A.4. (contd)

\begin{tabular}{|c|c|c|c|c|c|}
\hline $\begin{array}{l}\text { Revegetation } \\
\text { Unit }\end{array}$ & Subunit & $\begin{array}{l}\text { Island ID } \\
\text { Numbers }\end{array}$ & $\begin{array}{l}\text { Growth } \\
\text { Habit }\end{array}$ & Scientific Name & $\begin{array}{l}\text { Average } \\
\text { Number of } \\
\text { Seeds per } \\
\text { Island }\end{array}$ \\
\hline & \multirow{10}{*}{$\begin{array}{c}6636 \\
\text { Communication } \\
\text { towers }\end{array}$} & \multirow{10}{*}{$\begin{array}{c}278 \text { and } \\
279\end{array}$} & \multirow[t]{7}{*}{ forb } & Agastache occidentalis & 50 \\
\hline & & & & Astragalus purshii & 50 \\
\hline & & & & Erigeron linearis & 50 \\
\hline & & & & Erigeron poliospermus $^{(\mathrm{i})}$ & 50 \\
\hline & & & & $\begin{array}{l}\text { Eriophyllum lanatum var. } \\
\text { integrifolum }\end{array}$ & 50 \\
\hline & & & & Lomatium grayi & 50 \\
\hline & & & & Sedum leibergii & 50 \\
\hline & & & \multirow{3}{*}{$\begin{array}{c}\text { shrub } \\
\text { subshrub }\end{array}$} & Tetradymia canescens & 50 \\
\hline & & & & Eriogonum sphaerocephalum & 50 \\
\hline & & & & Nestotus stenophyllus & 50 \\
\hline
\end{tabular}

(a) The number of seeds planted at each island was averaged across each sub unit. Values for smaller seeded species were estimated by weight and known weight-to-seed-count ratios.

(b) Island 1572 was planted with 2284 Erigeron filifolius seeds; not used to calculate average.

(c) 1587 upslope island only.

(d) 1588 down-island slope only.

(e) Islands 269 and 270 only.

(f) Islands $269,270,272$, and 273 only.

(g) Islands 266 and 267 only.

(h) Island 268 only.

(i) Island 279 only. 


\section{Appendix B}

\section{Plot Locations and Photographs}




\section{Appendix B}

\section{Plot Locations and Photographs}

Table B.1. Modified Whittaker Plot IDs and Locations (Coordinate system: Washington State Plane [South]; Datum: NAD83; Units: meters)

\begin{tabular}{|c|c|c|c|c|c|c|}
\hline Unit & Sub Unit & $\begin{array}{l}\text { Plot ID } \\
\text { Number }\end{array}$ & $\begin{array}{l}\text { Point A } \\
\text { Northing }\end{array}$ & $\begin{array}{l}\text { Point A } \\
\text { Easting }\end{array}$ & $\begin{array}{l}\text { Bearing } \\
\text { from } \\
\text { Point A to } \\
\text { B }\end{array}$ & Plot Shape and Comment \\
\hline \multirow[t]{2}{*}{ Reference } & 1200-ft Road & 1200-REF-1 & 118982.858 & 573326.294 & $315^{\circ}$ & $20-\mathrm{m} \times 5-\mathrm{m}$ plot \\
\hline & Rattlesnake Ridge & RR-1 & 118791.649 & 569113.025 & $0^{\circ}$ & $20-\mathrm{m} \times 5-\mathrm{m}$ plot \\
\hline \multirow[t]{3}{*}{1} & Rattlesnake Springs & 1-RS-1 & 130727.718 & 561134.148 & $36^{\circ}$ & $20-\mathrm{m} \times 5-\mathrm{m}$ plot \\
\hline & & 1-RS-2 & 130706.044 & 561149.306 & $344^{\circ}$ & $33.3-\mathrm{m} \times 3-\mathrm{m}$ plot \\
\hline & & $1-\mathrm{RS}-3$ & 130658.486 & 561175.300 & $330^{\circ}$ & $33.3-\mathrm{m} \times 3-\mathrm{m}$ plot \\
\hline \multirow[t]{13}{*}{2} & ALE Headquaters & 2-HQ-1 & 118077.792 & 574149.020 & $274^{\circ}$ & $20-\mathrm{m} \times 5-\mathrm{m}$ plot \\
\hline & & 2-HQ-2 & 118097.713 & 574149.081 & $312^{\circ}$ & $20-\mathrm{m} \times 5-\mathrm{m}$ plot \\
\hline & & 2-HQ-3 & 118120.180 & 574122.040 & $292^{\circ}$ & $20-\mathrm{m} \times 5-\mathrm{m}$ plot \\
\hline & Hodges Well & 2-HW-1 & 116016.141 & 574390.988 & $148^{\circ}$ & $20-\mathrm{m} \times 5-\mathrm{m}$ plot \\
\hline & & 2-HW-2 & 116011.488 & 574385.539 & $168^{\circ}$ & $20-\mathrm{m} \times 5-\mathrm{m}$ plot \\
\hline & Debris Site 50 & $2-50-1$ & 118022.349 & 574682.013 & $250^{\circ}$ & $\begin{array}{l}33.3-\mathrm{m} \times 3-\mathrm{m} \text { plot includes } \\
\text { debris site and portion of road }\end{array}$ \\
\hline & Debris Site 60 & 2-DS60-Xa & 118332.490 & 572935.087 & $\begin{array}{l}\text { A-C: } 74^{\circ} \\
\text { B-D: } 350^{\circ}\end{array}$ & $\begin{array}{l}6 \text { systematic, } 1-\mathrm{m} \times 0.5 \mathrm{~m} \\
\text { quadrats }(3 \text { along each line) } \\
\text { sampled along cross-transect } \\
\text { lines at } 3,6 \text {, and } 9 \text { meters. } \\
\text { The side of the line (left or } \\
\text { right) was determined } \\
\text { randomly by tossing a coin. } \\
\text { A-C }=11 \mathrm{~m}, \mathrm{~B}-\mathrm{D}=13 \mathrm{~m} .\end{array}$ \\
\hline & & 2-DS60-Xb & 118340.754 & 572966.202 & $\begin{array}{l}\text { A-C: } 76^{\circ} \\
\text { B-D: } 342^{\circ}\end{array}$ & $\begin{array}{l}6 \text { systematic, } 1-\mathrm{m} \times 0.5 \mathrm{~m} \\
\text { quadrats ( } 3 \text { along each line) } \\
\text { sampled along cross-transect } \\
\text { lines at } 3,6 \text {, and } 8 \text { meters. } \\
\text { The side of the line (left or } \\
\text { right) was determined } \\
\text { randomly by tossing a coin. } \\
\text { A-C }=9.65 \mathrm{~m}, \mathrm{~B}-\mathrm{D}=9.2 \mathrm{~m} \text {. }\end{array}$ \\
\hline & Debris Site 109 & $2-109-1$ & 119304.831 & 572128.140 & not avail. & $\begin{array}{l}20-\mathrm{m} \times 5-\mathrm{m} \text { plot. Bearing } \\
\text { from point } \mathrm{A} \text { to } \mathrm{B} \text { is } \\
\text { downslope. }\end{array}$ \\
\hline & & $2-109-2$ & 119507.671 & 572112.077 & $229^{\circ}$ & $\begin{array}{l}33.3-\mathrm{m} \times 3-\mathrm{m} \text { plot on upper } \\
\text { road }\end{array}$ \\
\hline & & $2-109-3$ & 119836.982 & 572544.682 & $51^{\circ}$ & $\begin{array}{l}33.3-\mathrm{m} \times 3-\mathrm{m} \text { plot on lower } \\
\text { road }\end{array}$ \\
\hline & Debris Site 138 & $2-138-1$ & 120647.157 & 572978.47 & center point & $\begin{array}{l}4 \text { random, } 1 / 2-m \text { plot frames } \\
\text { ( } 1 \text { in each quadrant) }\end{array}$ \\
\hline & Debris Site 139 & $2-139-1$ & 120671.68 & 573004.39 & center point & $\begin{array}{l}\text { 7, 1/2-m plot frames randomly } \\
\text { sampled }\end{array}$ \\
\hline
\end{tabular}


Table B.1. (contd)

\begin{tabular}{|c|c|c|c|c|c|c|}
\hline Unit & Sub Unit & $\begin{array}{l}\text { Plot ID } \\
\text { Number }\end{array}$ & $\begin{array}{l}\text { Point A } \\
\text { Northing }\end{array}$ & $\begin{array}{l}\text { Point A } \\
\text { Easting }\end{array}$ & $\begin{array}{l}\text { Bearing } \\
\text { from } \\
\text { Point A to } \\
\text { B }\end{array}$ & Plot Shape and Comment \\
\hline & Debris Site 146 & 2-DS146-X & 121391.298 & 571205.866 & not avail. & $\begin{array}{l}12 \text { systematic, } 1 / 2-\mathrm{m} \text { quadrats } \\
\text { ( } 6 \text { along on each line) placed } \\
\text { every } 2 \mathrm{~m} \text {, coin toss } \\
\text { determined which side of the } \\
\text { line each quadrat would be } \\
\text { placed. } 17-\mathrm{m} \text { line (a to c), } 15 \text { - } \\
\mathrm{m} \text { line (b to d) }\end{array}$ \\
\hline \multirow[t]{3}{*}{3} & \multirow[t]{3}{*}{ Radio-telescope } & 3-RT-1 & 117915.745 & 570183.824 & $350^{\circ}$ & $20-\mathrm{m} \times 5-\mathrm{m}$ plot \\
\hline & & 3-RT-2 & 117853.599 & 570183.754 & $178^{\circ}$ & $33.3-\mathrm{m} \times 3-\mathrm{m}$ plot along road \\
\hline & & 3-RT-3 & 117857.368 & 570186.828 & $120^{\circ}$ & $33.3-\mathrm{m} \times 3-\mathrm{m}$ plot along road \\
\hline \multirow[t]{8}{*}{4} & \multirow[t]{2}{*}{$623 \mathrm{~A}$} & $4-623 \mathrm{~A}-1$ & 118007.498 & 570047.091 & $288^{\circ}$ & $20-\mathrm{m} \times 5-\mathrm{m}$ plot \\
\hline & & 4-623A-road & & & $\mathrm{n} / \mathrm{a}$ & $\begin{array}{l}6 \text { random, } 1 / 2-m \text { rectangles } \\
\text { along roadway }\end{array}$ \\
\hline & \multirow{3}{*}{$\begin{array}{l}\text { 6652-C Building } \\
\text { (barracks) }\end{array}$} & $4-\mathrm{C}-1$ & 118393.286 & 569540.284 & $67^{\circ}$ & $20-\mathrm{m} \times 5-\mathrm{m}$ plot \\
\hline & & $4-\mathrm{C}-2$ & 118424.672 & 569552.093 & $213^{\circ}$ & $20-\mathrm{m} \times 5-\mathrm{m}$ plot \\
\hline & & $4-\mathrm{C}-3$ & 118403.652 & 569538.390 & $344^{\circ}$ & $20-\mathrm{m} \times 5-\mathrm{m}$ plot \\
\hline & \multirow[t]{3}{*}{$\mathrm{CCCF}$} & 4-CCF-1 & 118643.307 & 569239.409 & $302^{\circ}$ & $20-\mathrm{m} \times 5-\mathrm{m}$ plot \\
\hline & & 4-CCF-Berm & & & $\mathrm{n} / \mathrm{a}$ & $\begin{array}{l}6 \text { random, } 1 / 2-m \text { rectangles } \\
\text { along roadway }\end{array}$ \\
\hline & & 4-CCF-Road & & & $\mathrm{n} / \mathrm{a}$ & $\begin{array}{l}6 \text { random, } 1 / 2-m \text { rectangles } \\
\text { around berm }\end{array}$ \\
\hline \multirow[t]{2}{*}{5} & \multirow{2}{*}{$\begin{array}{l}\text { 6652-C Shed and } \\
\text { Berm Area }\end{array}$} & 5-OB-1 & 118427.562 & 569502.077 & $312^{\circ}$ & $20-\mathrm{m} \times 5-\mathrm{m}$ plot \\
\hline & & $5-\mathrm{OB}-2$ & 118426.134 & 569485.307 & $255^{\circ}$ & $20-\mathrm{m} \times 5-\mathrm{m}$ plot \\
\hline \multirow[t]{2}{*}{6} & Crown Castle & $6-6635-X$ & 119117.428 & 568774.218 & $47^{\circ}$ & $7-\mathrm{m} \times 15-\mathrm{m}$ plot \\
\hline & $\begin{array}{l}\text { Communication } \\
\text { towers }\end{array}$ & $6-6636-1$ & 119575.359 & 568366.085 & $50^{\circ}$ & $20-\mathrm{m} \times 5-\mathrm{m}$ plot \\
\hline 7 & Pumphouse & 7-PH-X & 119685.181 & 568699.399 & center point & $\begin{array}{l}12 \text { random, } 1 / 2-m \text { plot frames } \\
\text { ( } 3 \text { in each quadrant) }\end{array}$ \\
\hline
\end{tabular}


Table B.2. Diversity Island Plot Locations and ID Numbers. Coordinates for transplant islands were not available when this document was drafted. (Coordinate system: Washington State Plane [South]; Datum: NAD83; Units: meters)

\begin{tabular}{|c|c|c|c|c|c|}
\hline Unit & Sub Unit & Plot Type & $\begin{array}{l}\text { Plot ID } \\
\text { Number }\end{array}$ & $\begin{array}{l}\text { Center-Point } \\
\text { Northing }\end{array}$ & $\begin{array}{c}\text { Center-Point } \\
\text { Easting }\end{array}$ \\
\hline \multirow[t]{5}{*}{1} & \multirow[t]{5}{*}{ Rattlesnake Springs } & \multirow[t]{3}{*}{ seeding } & 1581 & 130733.934 & 561132.552 \\
\hline & & & 1582 & 130745.359 & 561133.969 \\
\hline & & & 1583 & 130739.705 & 561140.683 \\
\hline & & \multirow[t]{2}{*}{ transplant } & circle & & \\
\hline & & & road & & \\
\hline \multirow[t]{29}{*}{2} & \multirow[t]{14}{*}{ ALE HQ } & \multirow[t]{8}{*}{ seeding } & 1568 & 118161.582 & 574112.958 \\
\hline & & & 1569 & 118149.012 & 574103.938 \\
\hline & & & 1570 & 118121.470 & 574120.534 \\
\hline & & & 1571 & 118101.307 & 574097.819 \\
\hline & & & 1572 & 118084.911 & 574114.126 \\
\hline & & & 1573 & 118059.142 & 574125.525 \\
\hline & & & 1574 & 118073.137 & 574159.345 \\
\hline & & & 1575 & 118113.410 & 574156.782 \\
\hline & & \multirow[t]{6}{*}{ transplant } & 280 & & \\
\hline & & & 281 & & \\
\hline & & & 282 & & \\
\hline & & & 283 & & \\
\hline & & & 1697 & & \\
\hline & & & 1698 & & \\
\hline & \multirow[t]{3}{*}{ HodgesWell } & \multirow[t]{3}{*}{ seeding } & 1576 & 116010.488 & 574403.022 \\
\hline & & & 1577 & 115990.387 & 574391.298 \\
\hline & & & 1578 & 116021.973 & 574392.970 \\
\hline & Debris Site 50 & seeding & 1586 & 118015.867 & 574676.767 \\
\hline & Debris Site 60 (up slope) & seeding & 1587 & 118329.277 & 572940.087 \\
\hline & Debris Site 60 (down slope) & seeding & 1588 & 118340.273 & 572968.502 \\
\hline & Debris Site 109 (Wells) & seeding & 1585 & 119305.831 & 572139.590 \\
\hline & \multirow[t]{5}{*}{ Debris Site 109 (Road) } & \multirow[t]{5}{*}{ transplant } & 1 & & \\
\hline & & & 2 & & \\
\hline & & & 3 & & \\
\hline & & & 4 & & \\
\hline & & & 5 & & \\
\hline & Debris Site 138 & seeding & 1584 & 120669.637 & 573001.824 \\
\hline & \multirow[t]{2}{*}{ Debris Site 146} & \multirow[t]{2}{*}{ seeding } & 1579 & 121405.265 & 571209.233 \\
\hline & & & 1580 & 121395.810 & 571206.128 \\
\hline \multirow[t]{2}{*}{3} & \multirow[t]{2}{*}{ Radiotelescope } & seeding & 1589 & 117908.185 & 570186.073 \\
\hline & & transplant & 65 & 117933.626 & 570172.479 \\
\hline
\end{tabular}


Table B.2. (contd)

\begin{tabular}{|c|c|c|c|c|c|}
\hline Unit & Sub Unit & Plot Type & $\begin{array}{l}\text { Plot ID } \\
\text { Number }\end{array}$ & $\begin{array}{c}\text { Center-Point } \\
\text { Northing }\end{array}$ & $\begin{array}{c}\text { Center-Point } \\
\text { Easting }\end{array}$ \\
\hline \multirow[t]{18}{*}{4} & 6652-C Building & seeding & 259 & 118416.515 & 569512.910 \\
\hline & & & 260 & 118421.129 & 569521.330 \\
\hline & & & 261 & 118418.173 & 569529.151 \\
\hline & & & 262 & 118416.460 & 569537.108 \\
\hline & & & 263 & 118433.687 & 569544.846 \\
\hline & & & 264 & 118422.972 & 569552.808 \\
\hline & & & 265 & 118412.569 & 569563.661 \\
\hline & & transplant & 64 & & \\
\hline & & & 284 & & \\
\hline & & & 285 & & \\
\hline & & & 286 & & \\
\hline & & & 287 & & \\
\hline & & & 1592 & & \\
\hline & $\mathrm{CCCF}$ & seeding & 269 & 118658.283 & 569217.713 \\
\hline & & & 270 & 118655.222 & 569224.464 \\
\hline & & & 272 & 118652.929 & 569231.177 \\
\hline & & & 273 & 118648.635 & 569238.060 \\
\hline & & & 274 & 118643.389 & 569245.565 \\
\hline \multirow[t]{4}{*}{5} & 6652-C Shed and Berm & seeding & 266 & 118422.203 & 569474.166 \\
\hline & & & 267 & 118433.248 & 569470.196 \\
\hline & & & 268 & 118431.503 & 569481.915 \\
\hline & & transplant & 1798 & & \\
\hline \multirow[t]{8}{*}{6} & 6635 Crown Castle & seeding & 275 & 119108.813 & 568775.166 \\
\hline & & & 276 & 119112.564 & 568779.518 \\
\hline & & & 277 & 119112.280 & 568770.959 \\
\hline & & transplant & 66 & & \\
\hline & & & 1590 & & \\
\hline & 6636 Communication Towers & seeding & 278 & 119575.482 & 568362.351 \\
\hline & & & 279 & 119579.805 & 568366.957 \\
\hline & & transplant & 1591 & & \\
\hline 7 & 6652-T Pumphouse & transplant & $\begin{array}{l}288 \\
\text { flag }\end{array}$ & 119685.66 & 568699.77 \\
\hline
\end{tabular}




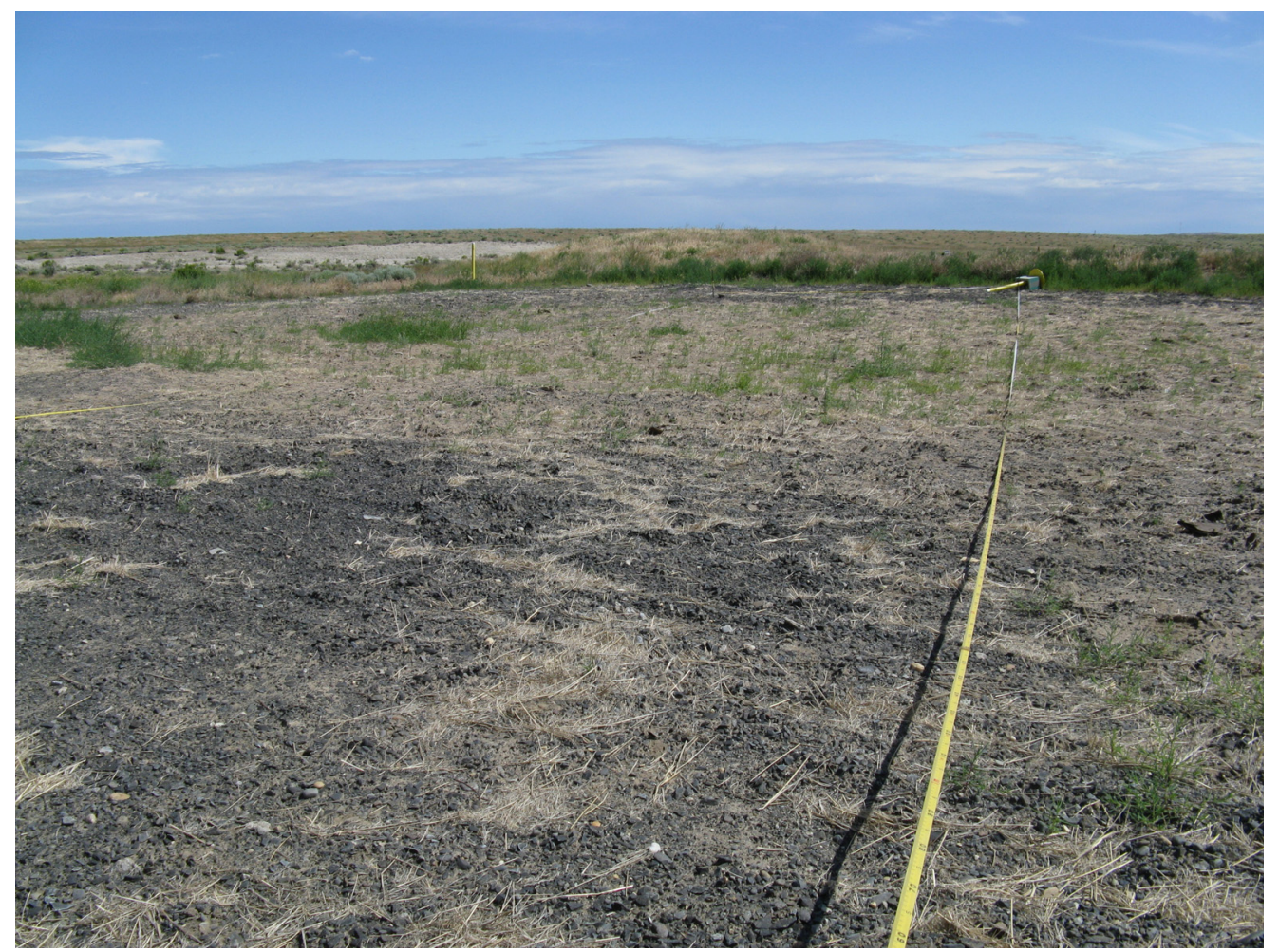

Figure B.1. Unit 1: Monitoring Plot 1-RS-1; Northing 130727.718; Easting 561134.148

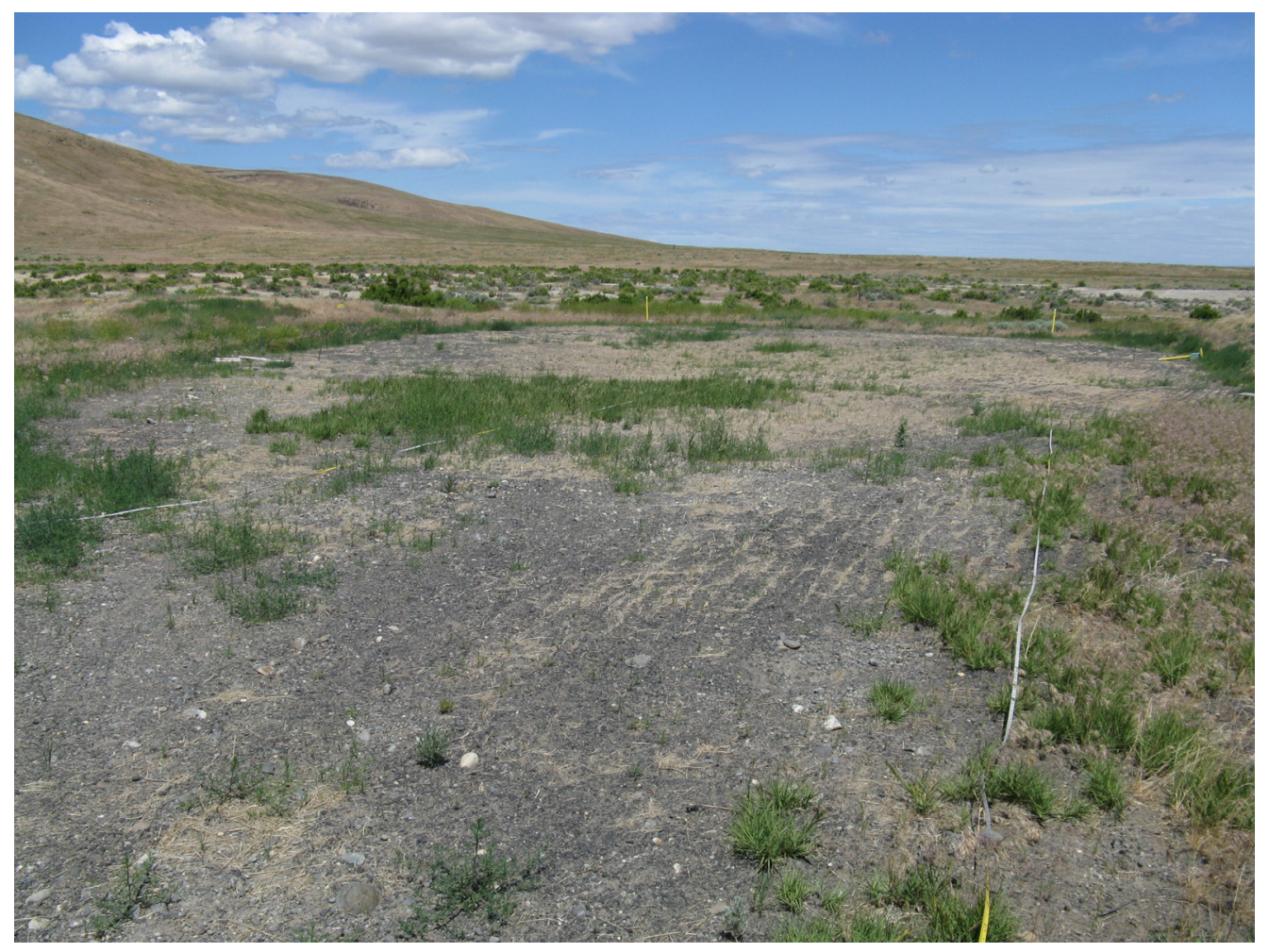

Figure B.2. Unit 1: Monitoring Plot 1-RS-2; Northing 130706.044; Easting 561149.306 


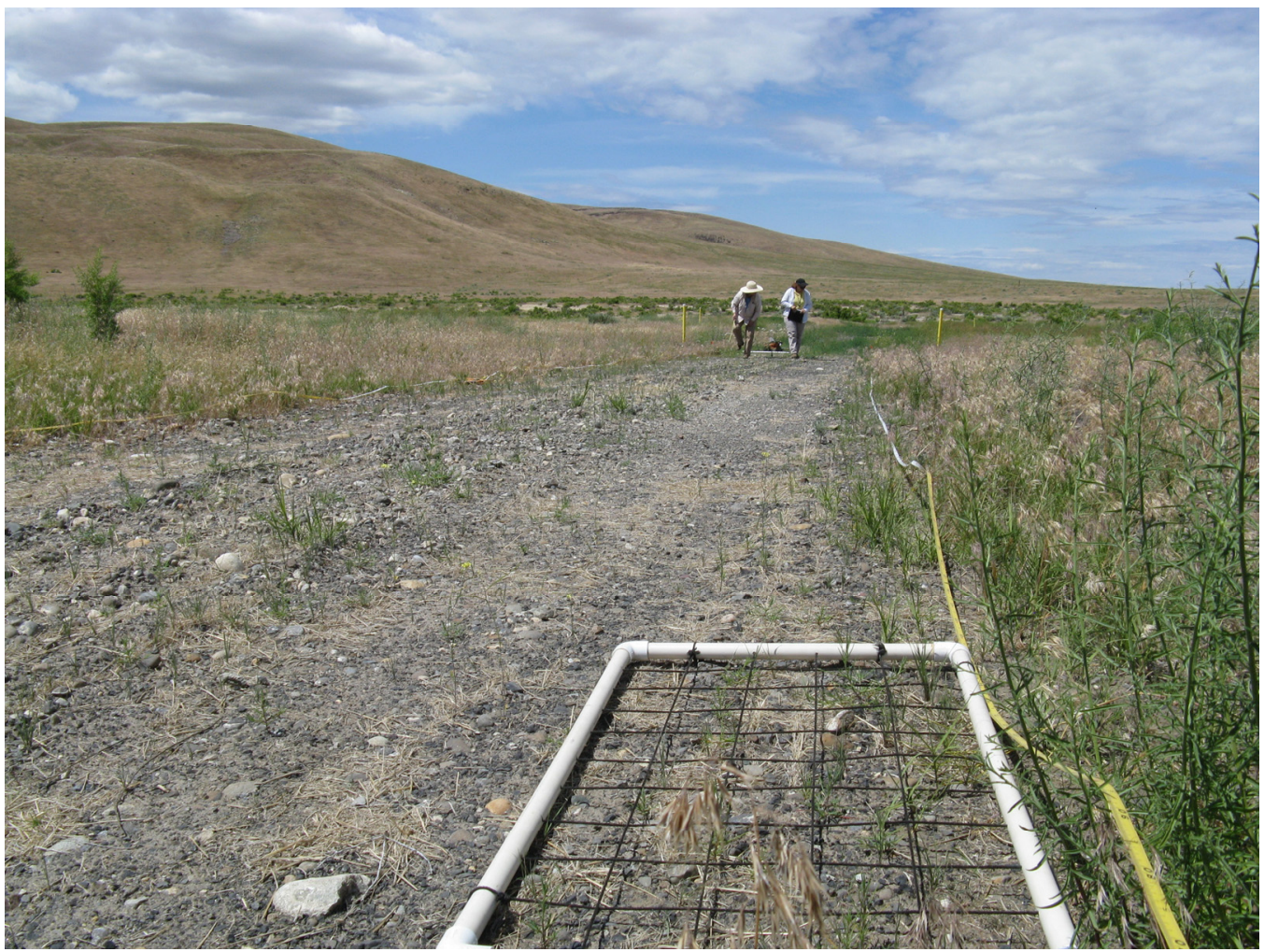

Figure B.3. Unit 1: Monitoring Plot 1-RS-3; Northing 130658.486, Easting 561175.300

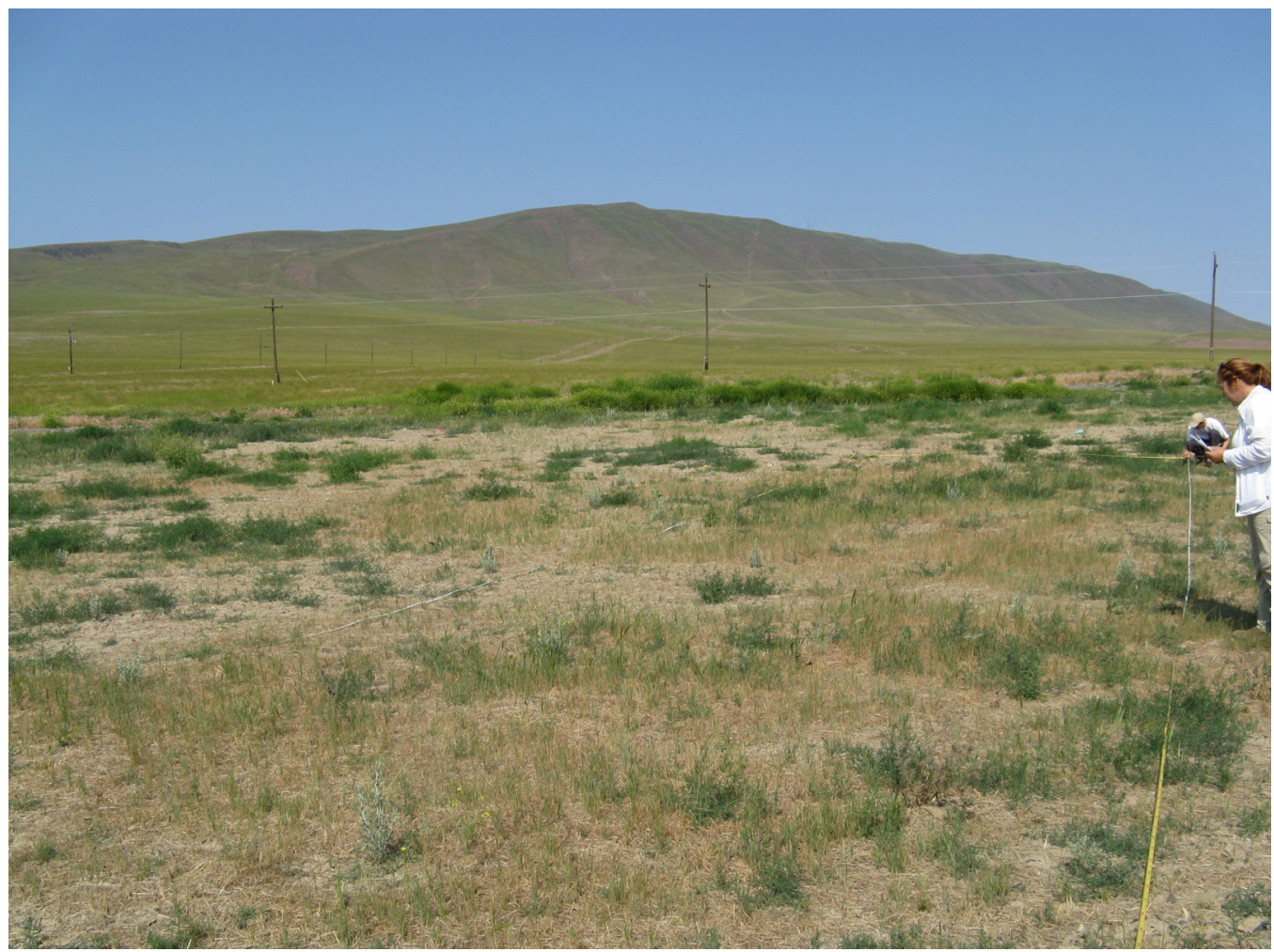

Figure B.4. Unit 2 (ALE HQ): Monitoring Plot 2-HQ-1; Starting Coordinate N 118076, E 574149 


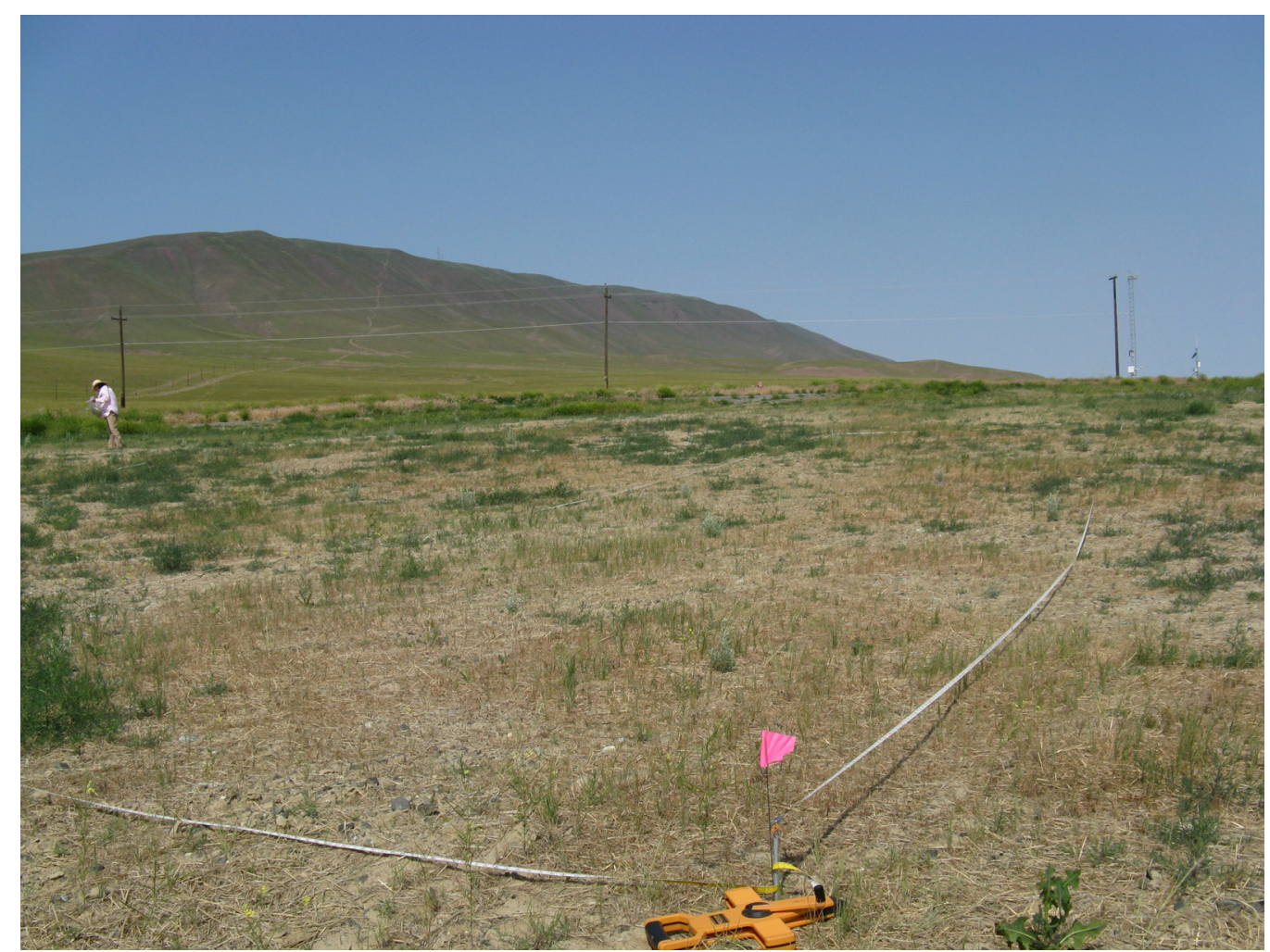

Figure B.5. Unit 2 (ALE HQ): Monitoring Plot 2-HQ-2; Starting Coordinate N118098.3, E 574149.5

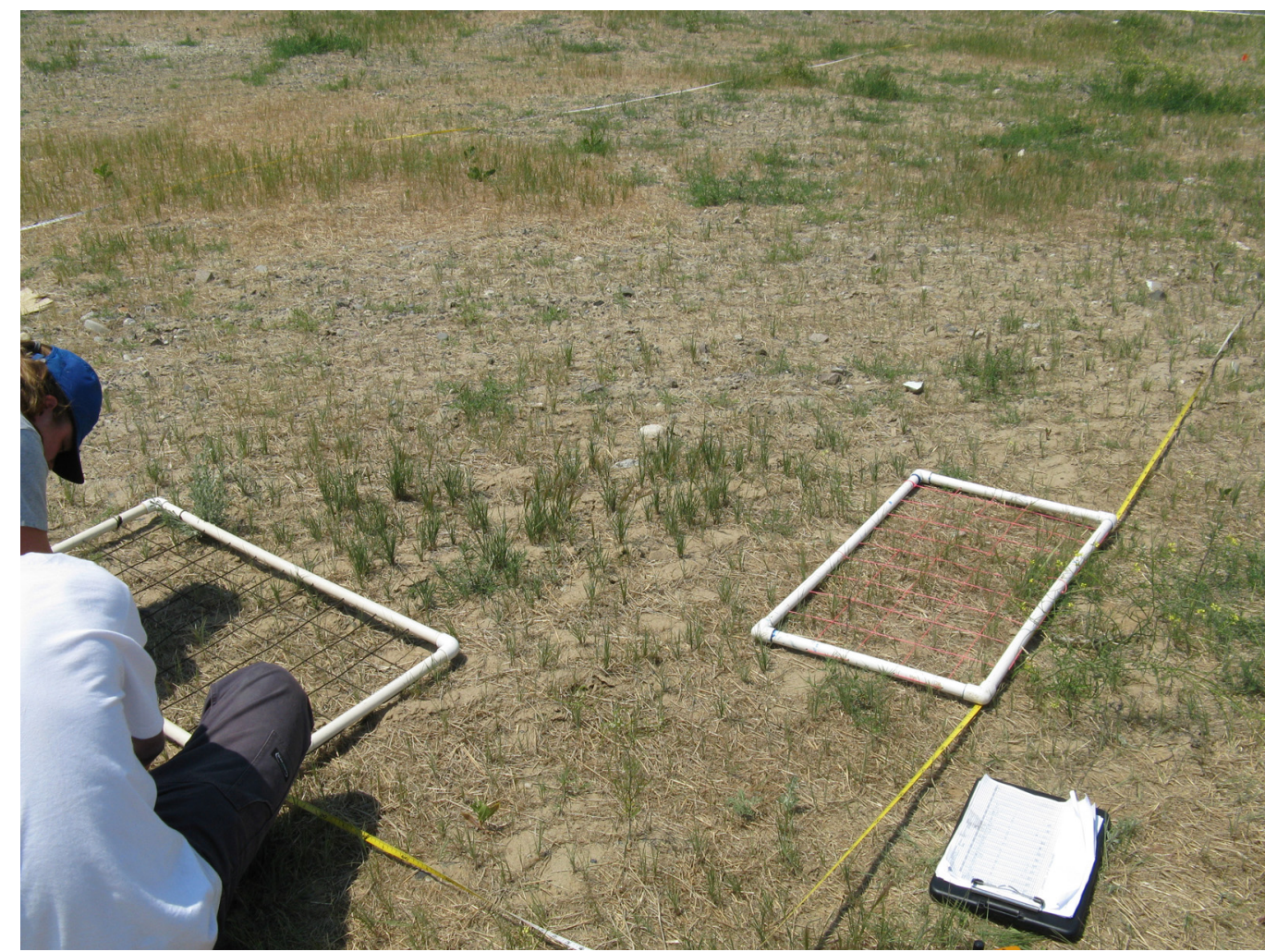

Figure B.6. Unit 2 (ALE HQ): Plot 2-HQ-3; Starting Coordinate N 118120.180, E 574122.040 


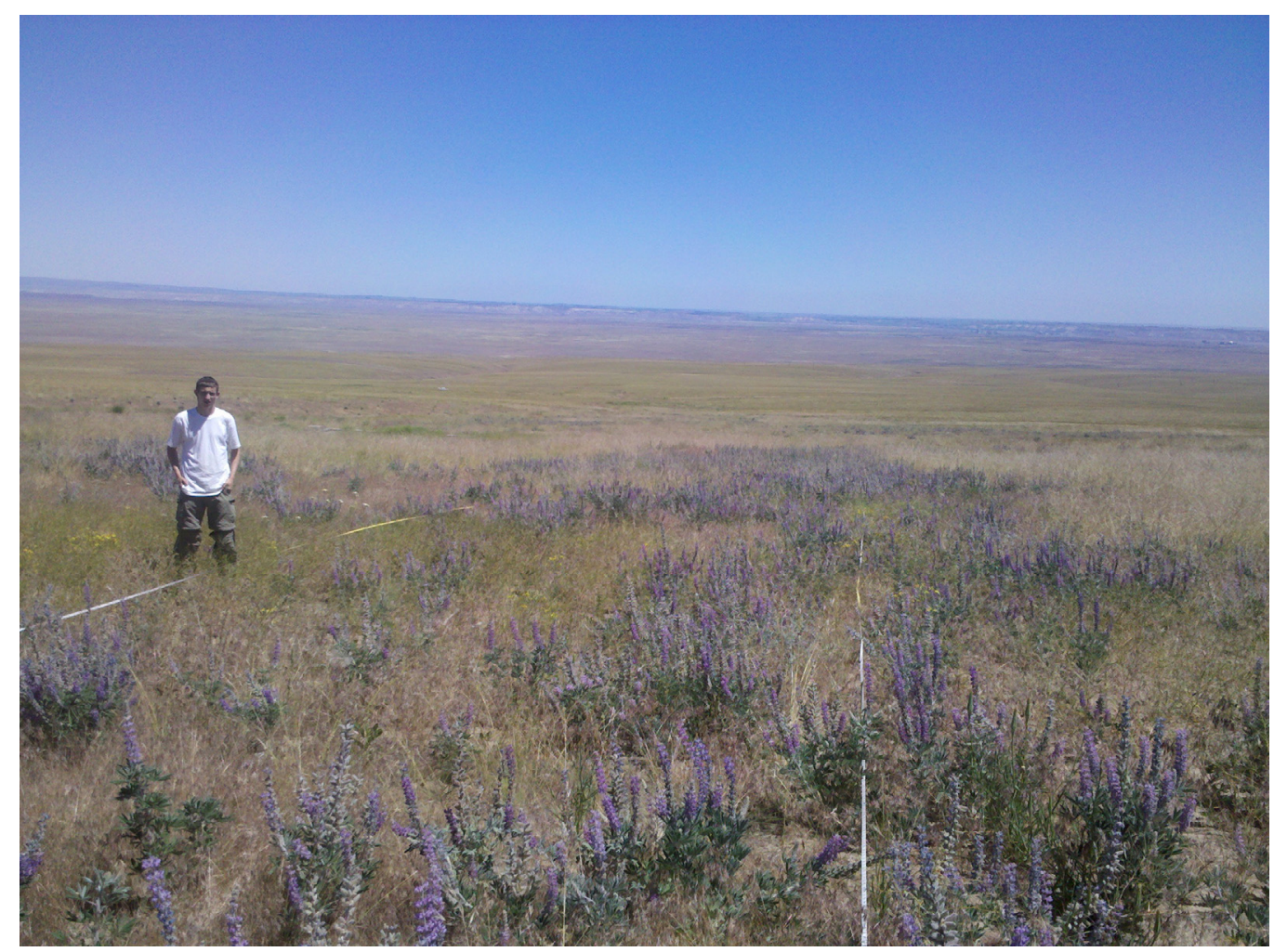

Figure B.7. Unit 2 (Debris Site 109): Monitoring Plot 2-109-1, Starting Coordinate N 119304.831, E 572128.140

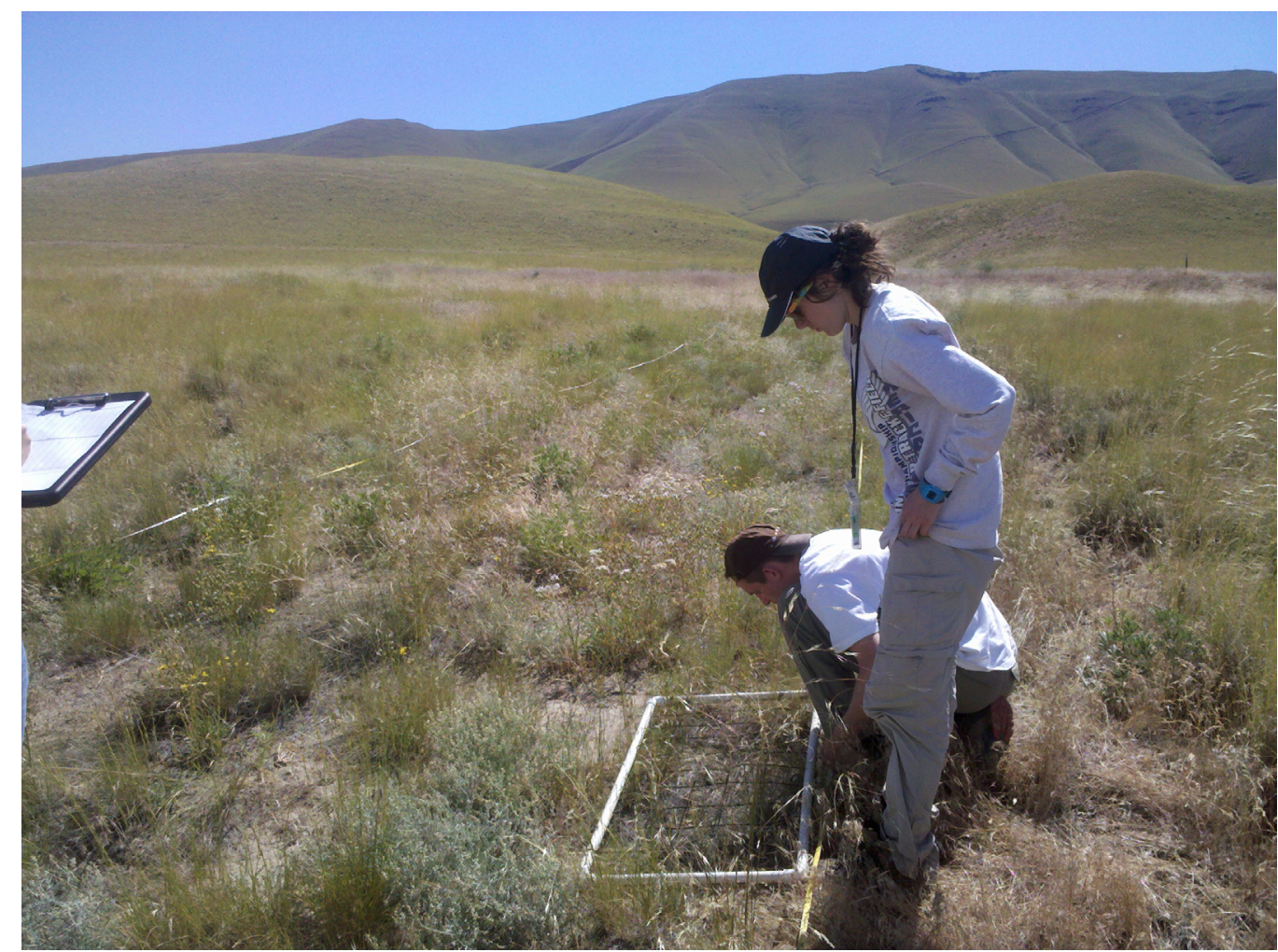

Figure B.8. Unit 2 (Debris Site 109): Plot 2-109-2, Starting Coordinates N 119507.671, E 572112.077 


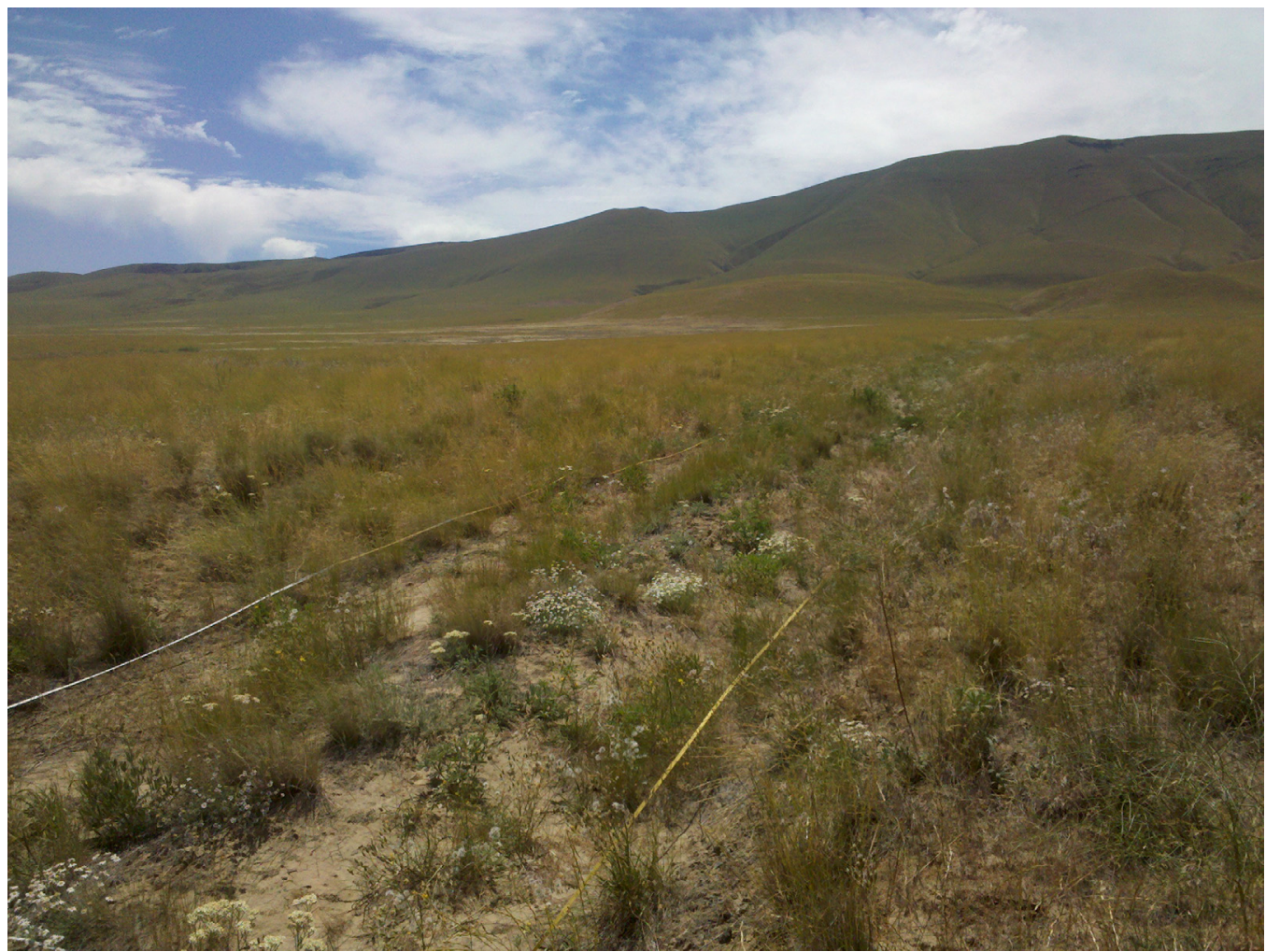

Figure B.9. Unit 2 (Debris Site 109): Plot 2-109-3, Starting Coordinates N 119835.11, E 572542.77

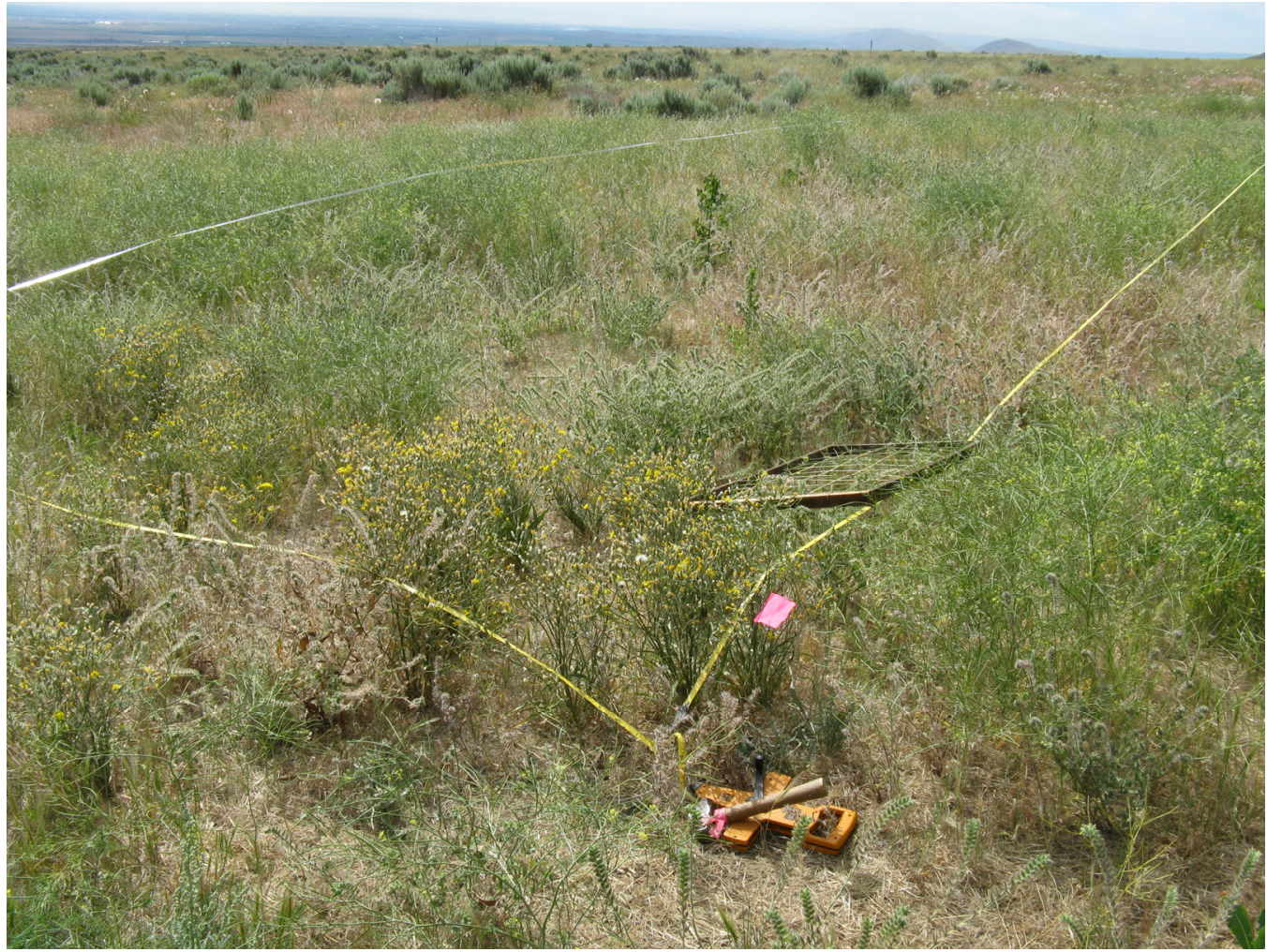

Figure B.10. Unit 2 (Hodges Well): Plot 2-HW-1, Starting Coordinates N 116016.141, E 574390.988 


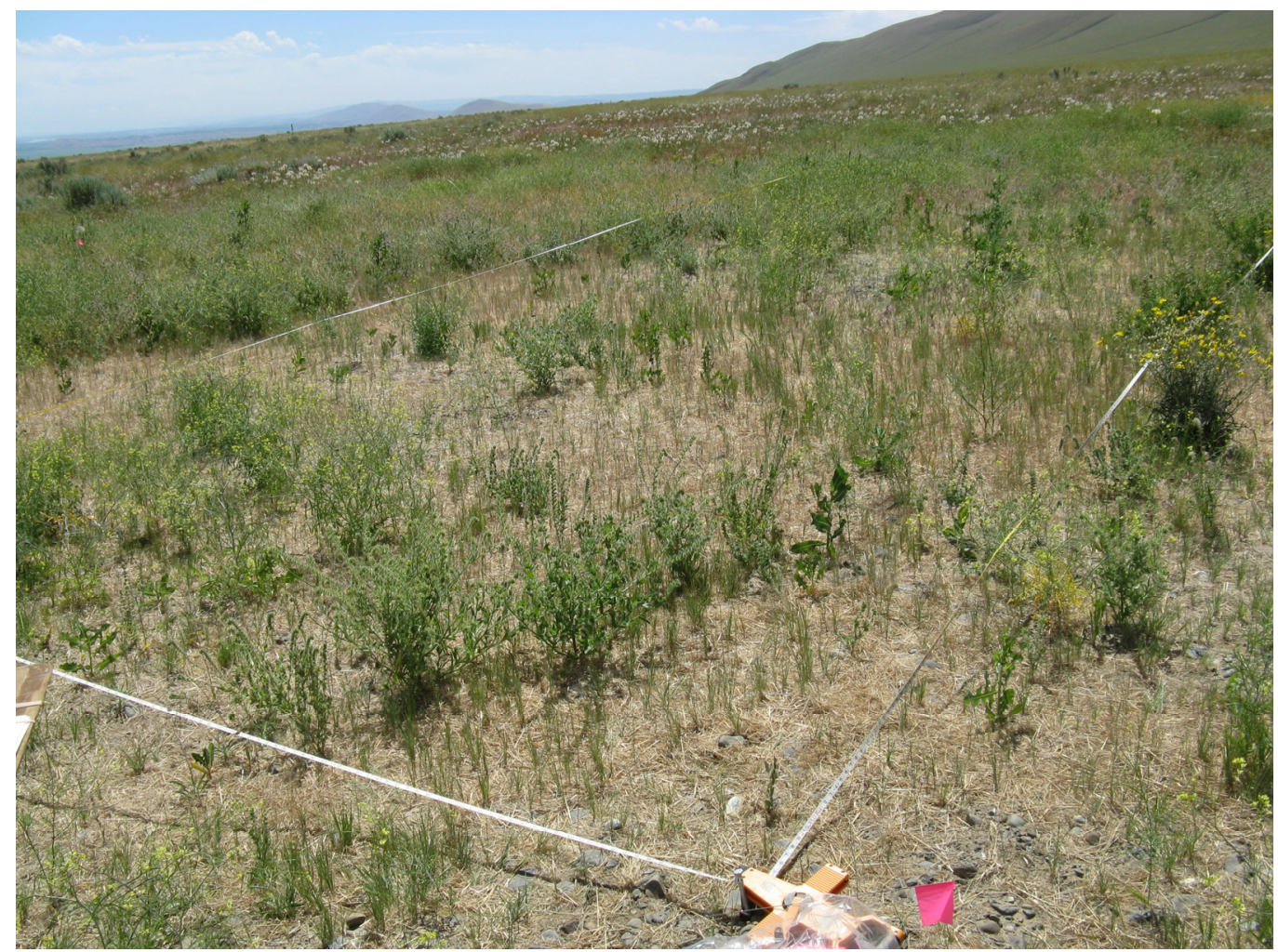

Figure B.11. Unit 2 (Hodges Well): Plot 2-HW-2, Starting Coordinates N 116011.488, E 574385.539

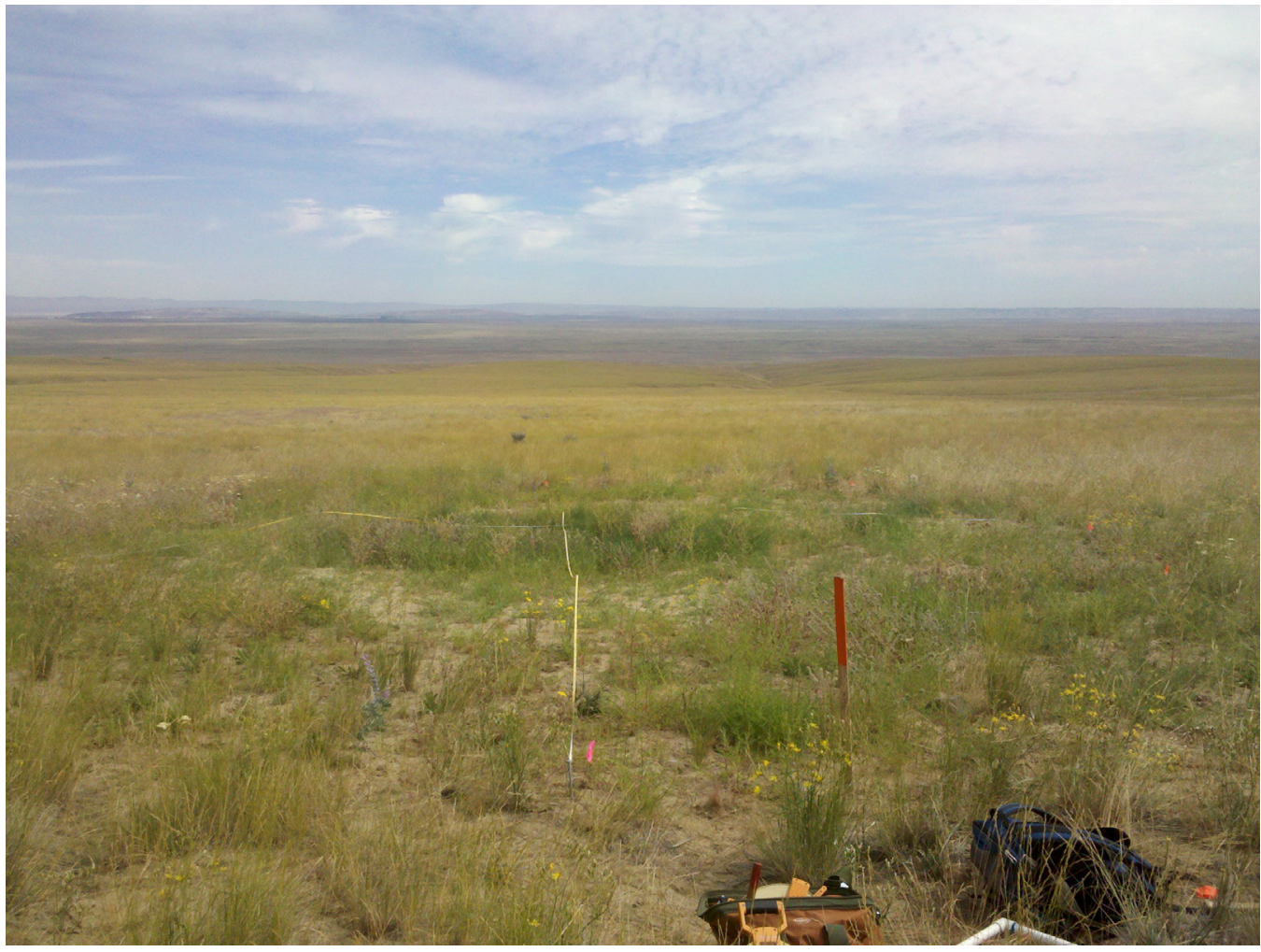

Figure B.12. Unit 2 (Debris Site 146): Plot 2-DS146-X, Starting Coordinates N 121391.15, E 511205.95 


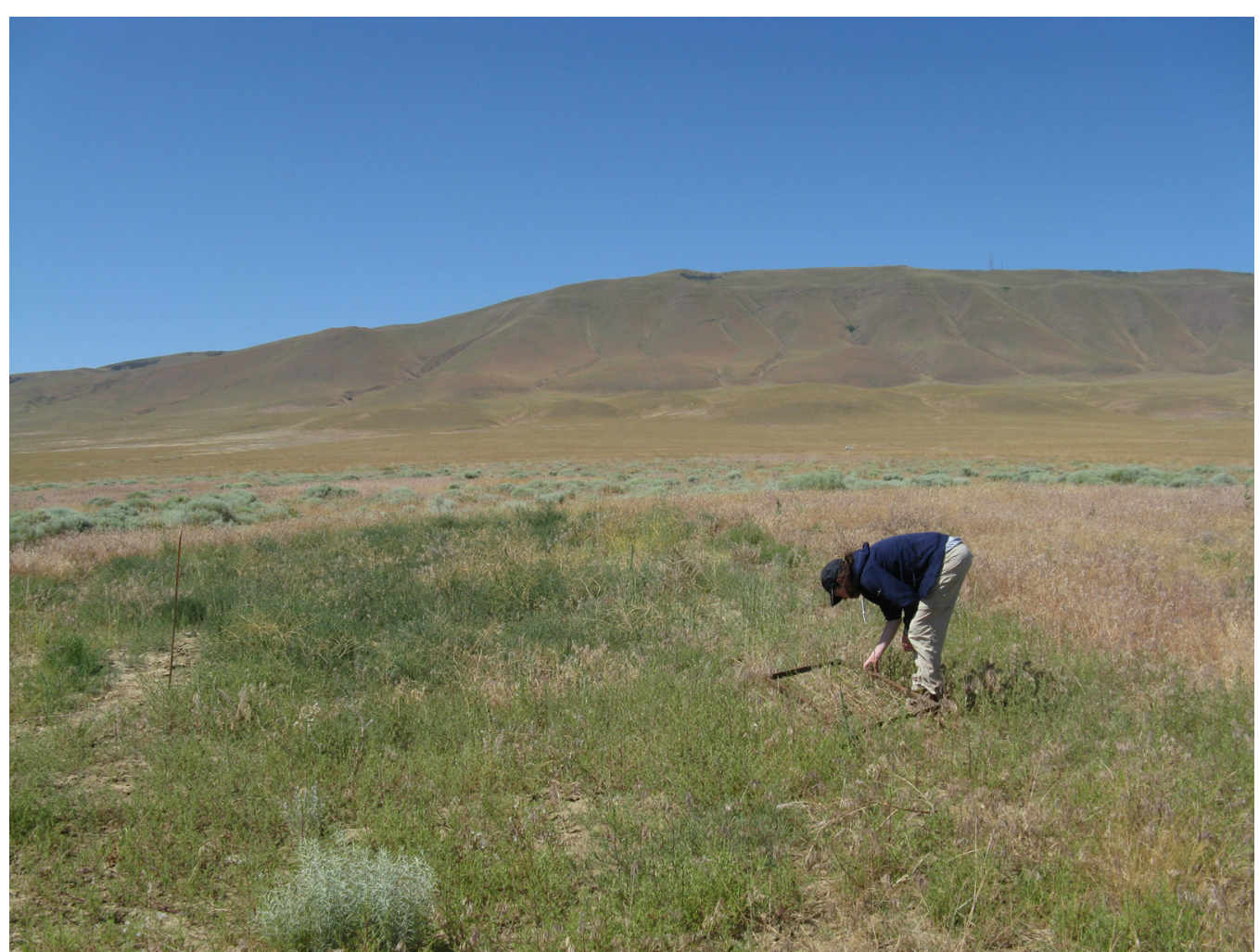

Figure B.13. Unit 2 (Debris Site 139): Plot 2-139-1, Starting Coordinates N 120672.508, E 573004.685

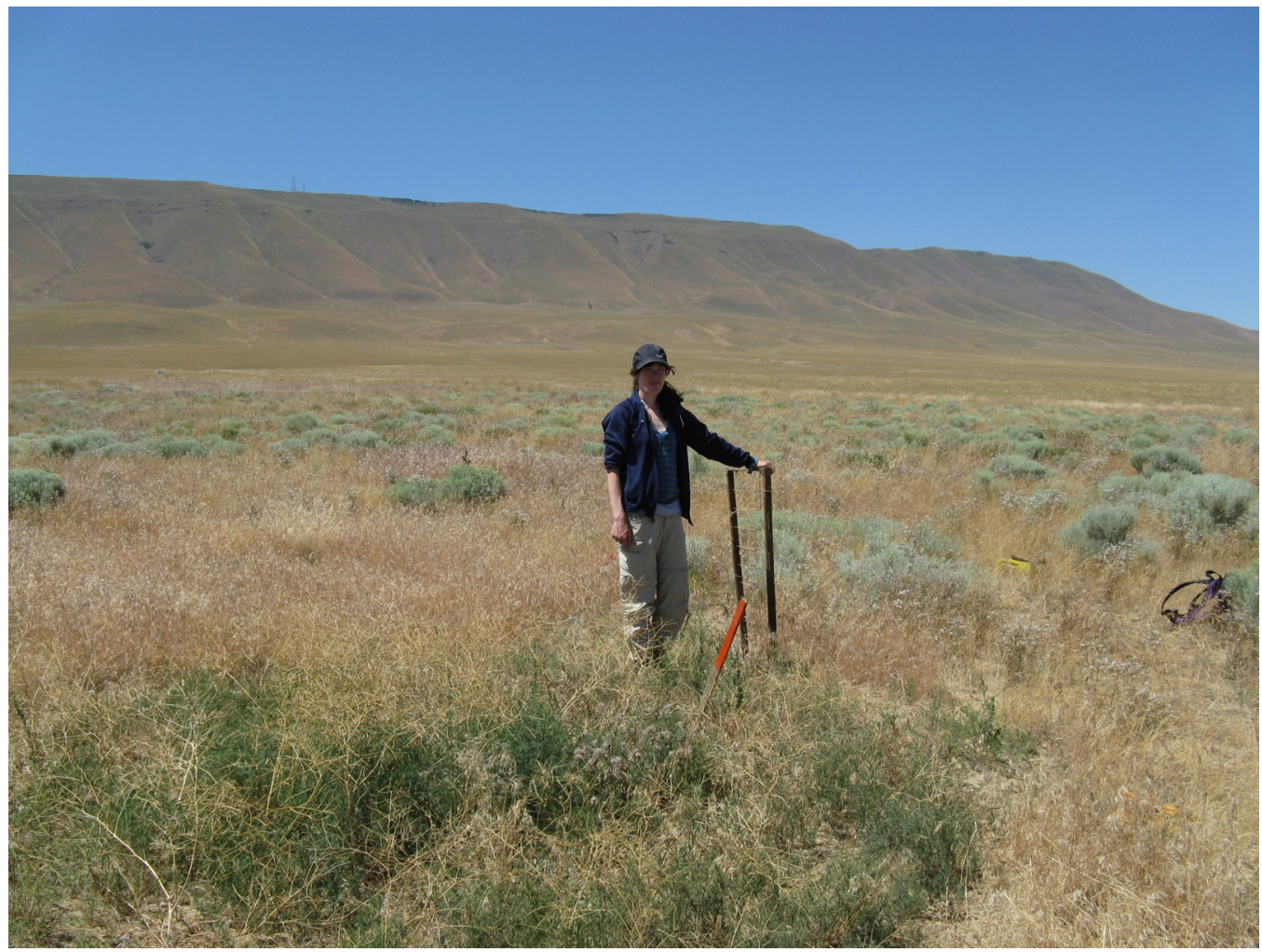

Figure B.14. Unit 2 (Debris Site 138): Plot 2-138-1, Starting Coordinates N 120647.157, E 572978.470 


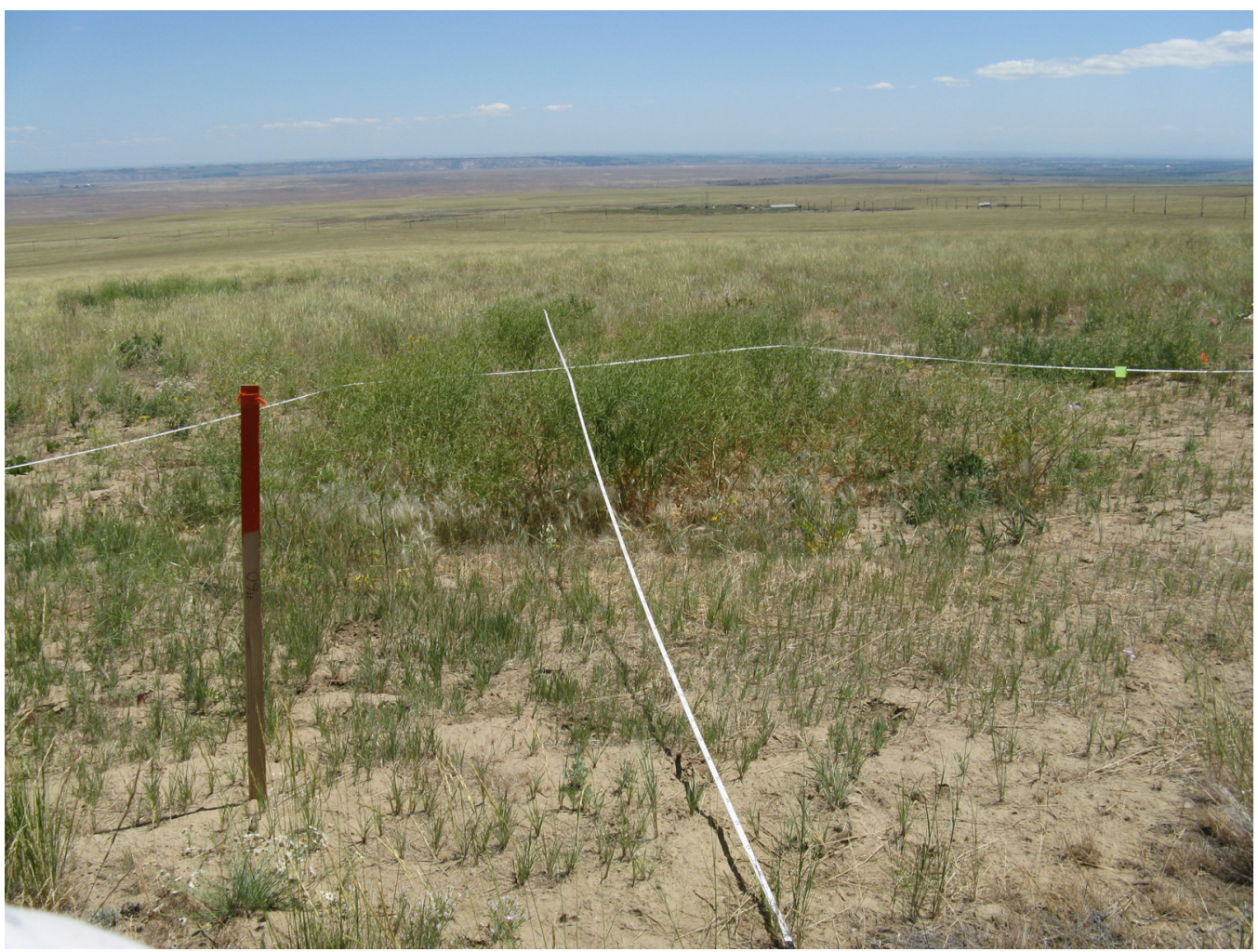

Figure B.15. Unit 2 (Debris Site 60): Plot 2-DS60-Xa, Starting Coordinates N118332.44, E 572935.5

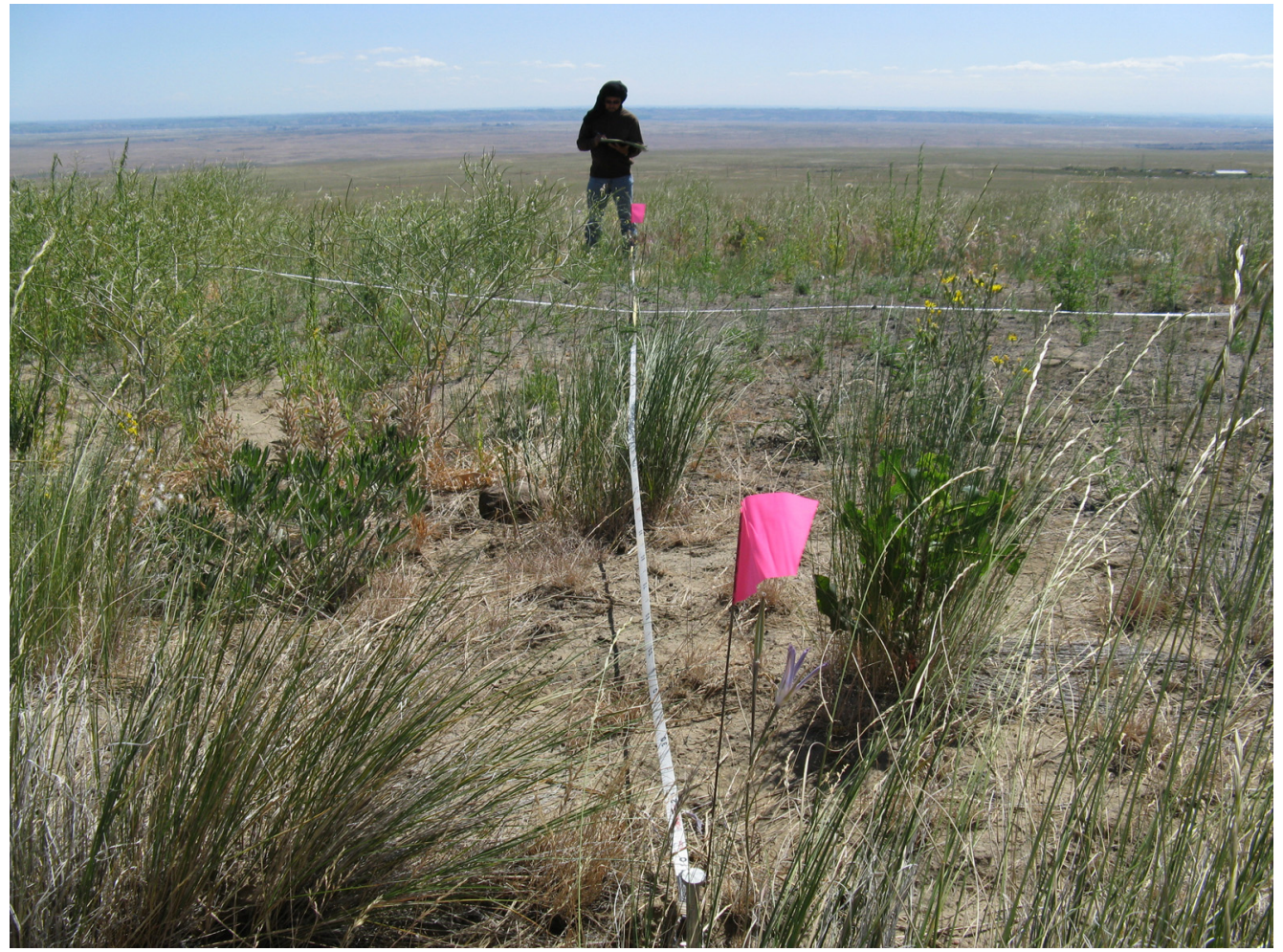

Figure B.16. Unit 2 (Debris Site 60): Plot 2-DS60-Xb, Starting Coordinates N 11830.56, E 572966.1 


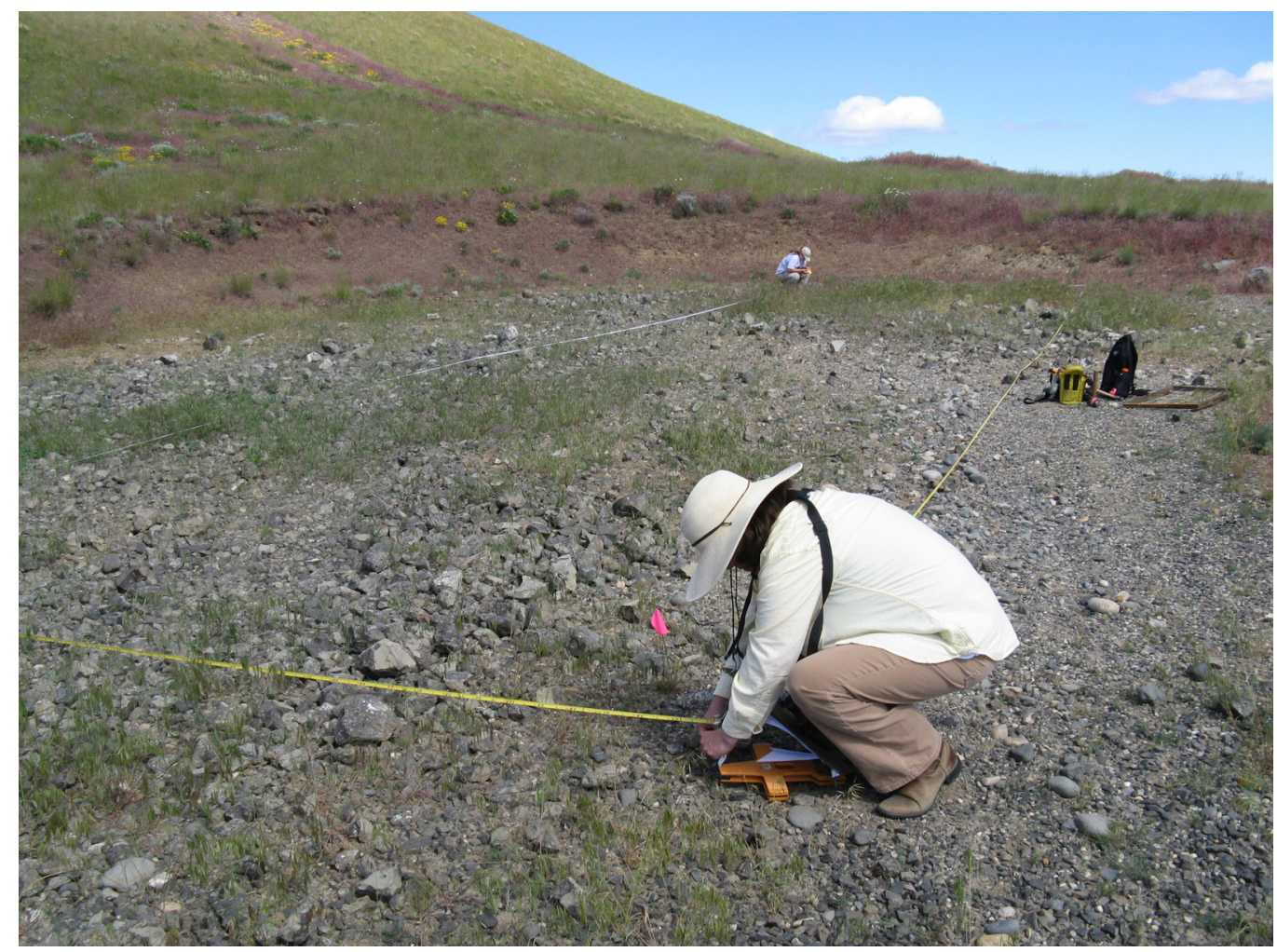

Figure B.17. Unit 3: Plot 3-RT-1, Starting Coordinates N 117915.745, E 570183.824

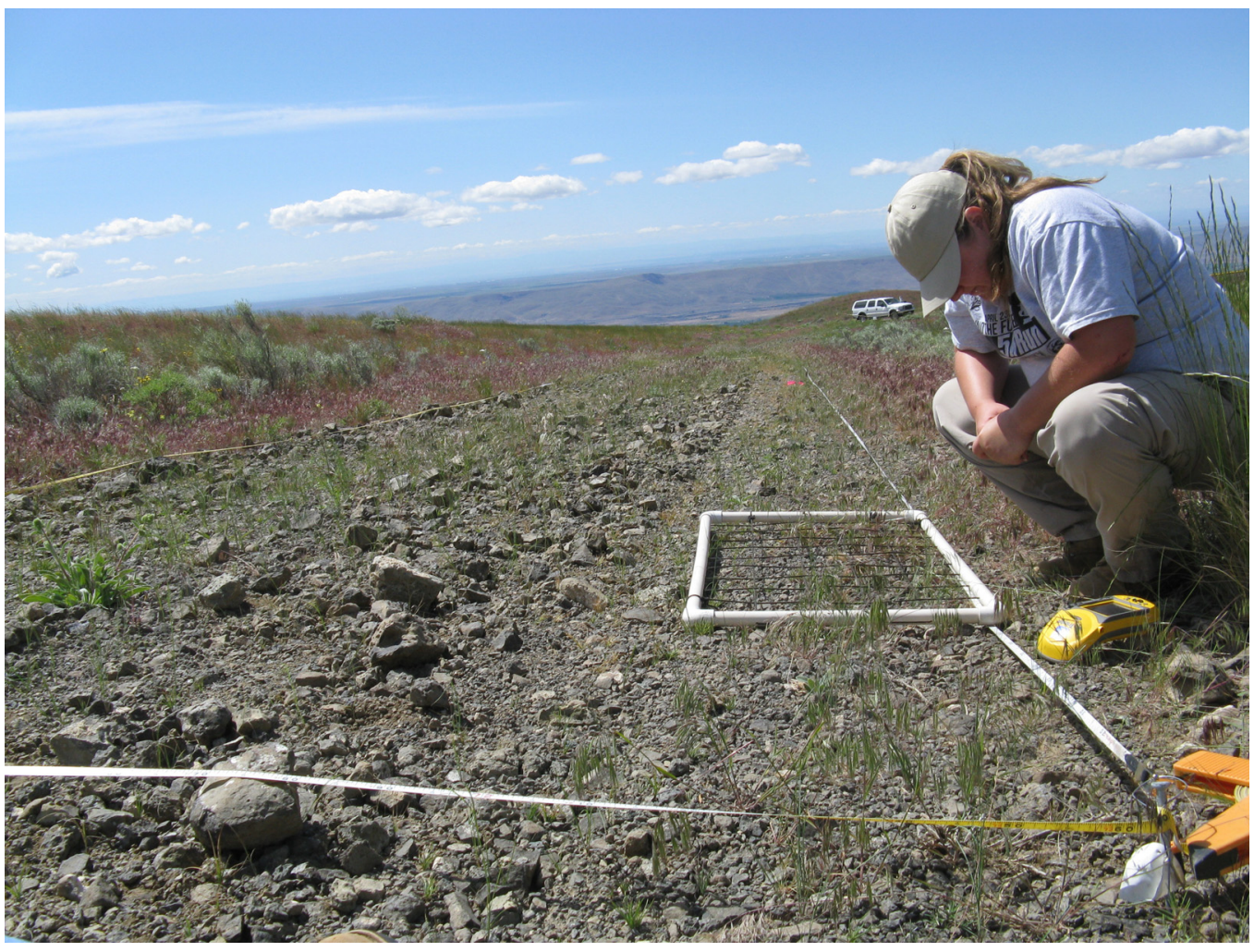

Figure B.18. Unit 3: Plot 3-RT-2, Starting Coordinates N 117852.03, E 570184.23 


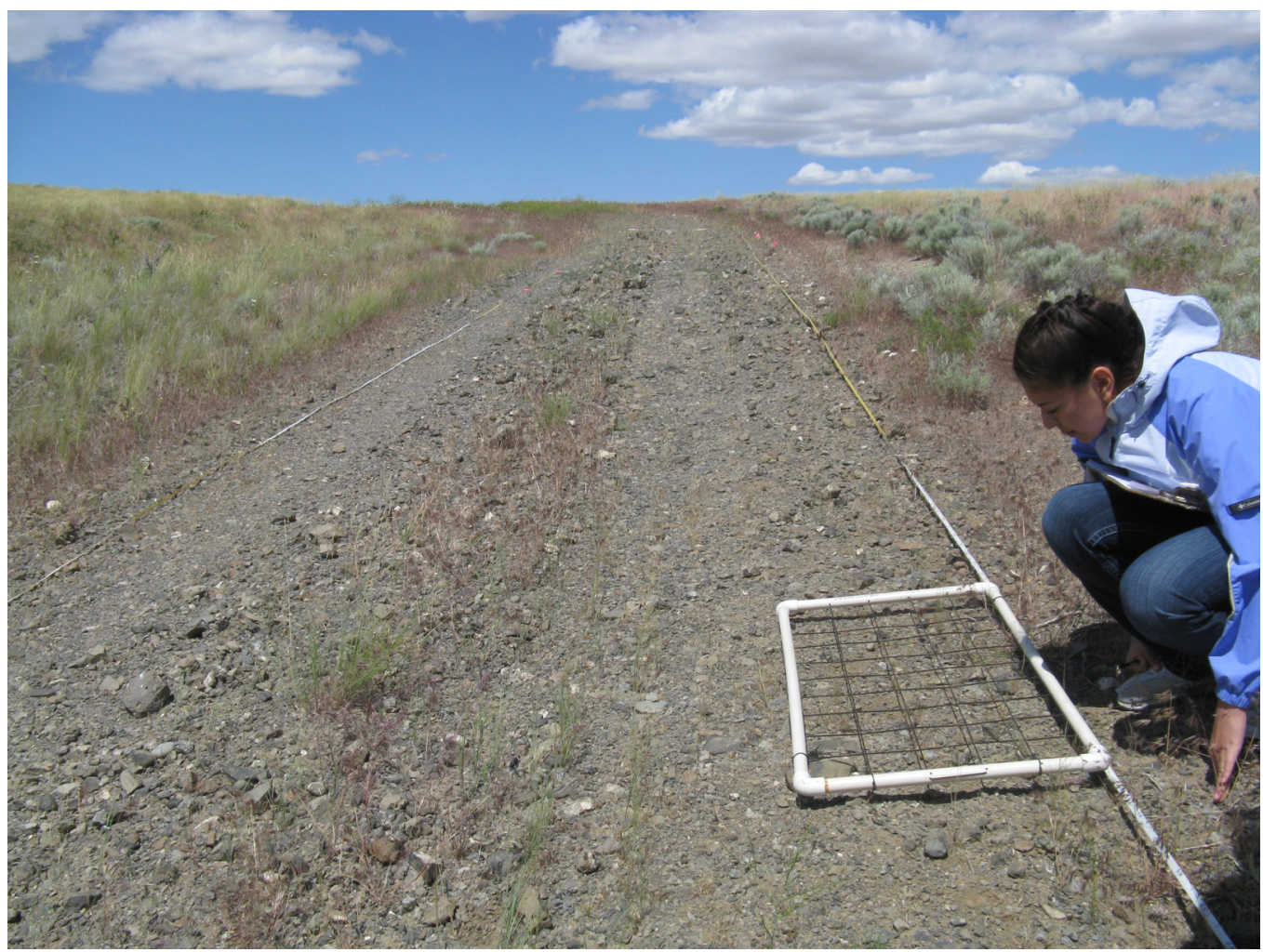

Figure B.19. Unit 3: Plot 3-RT-3, Starting Coordinates N 117857, E 570187

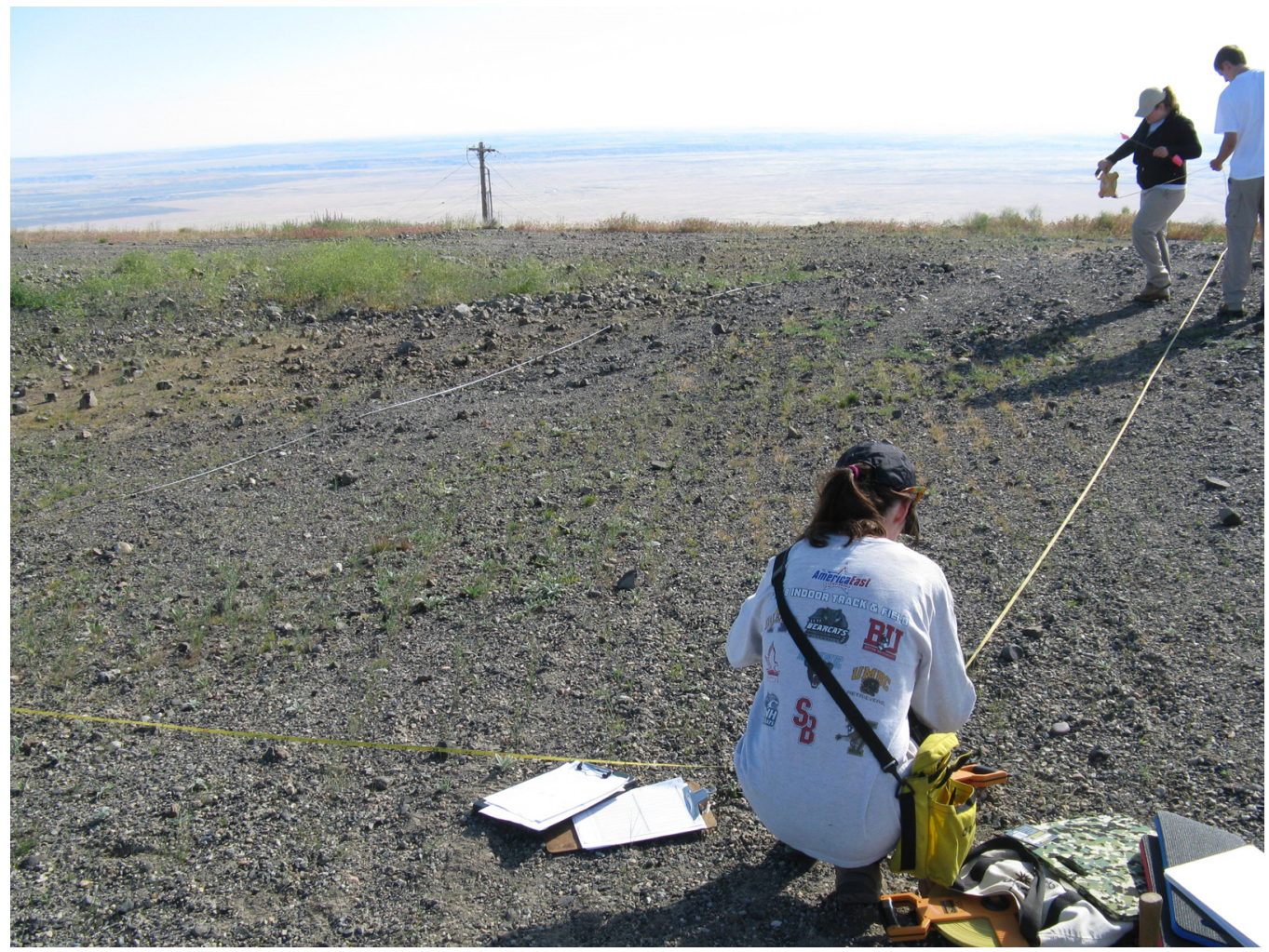

Figure B.20. Unit 4 (6652-C Bldgs): Plot 4-C-1, Starting Coordinates N 118594.6, E 569539.3 


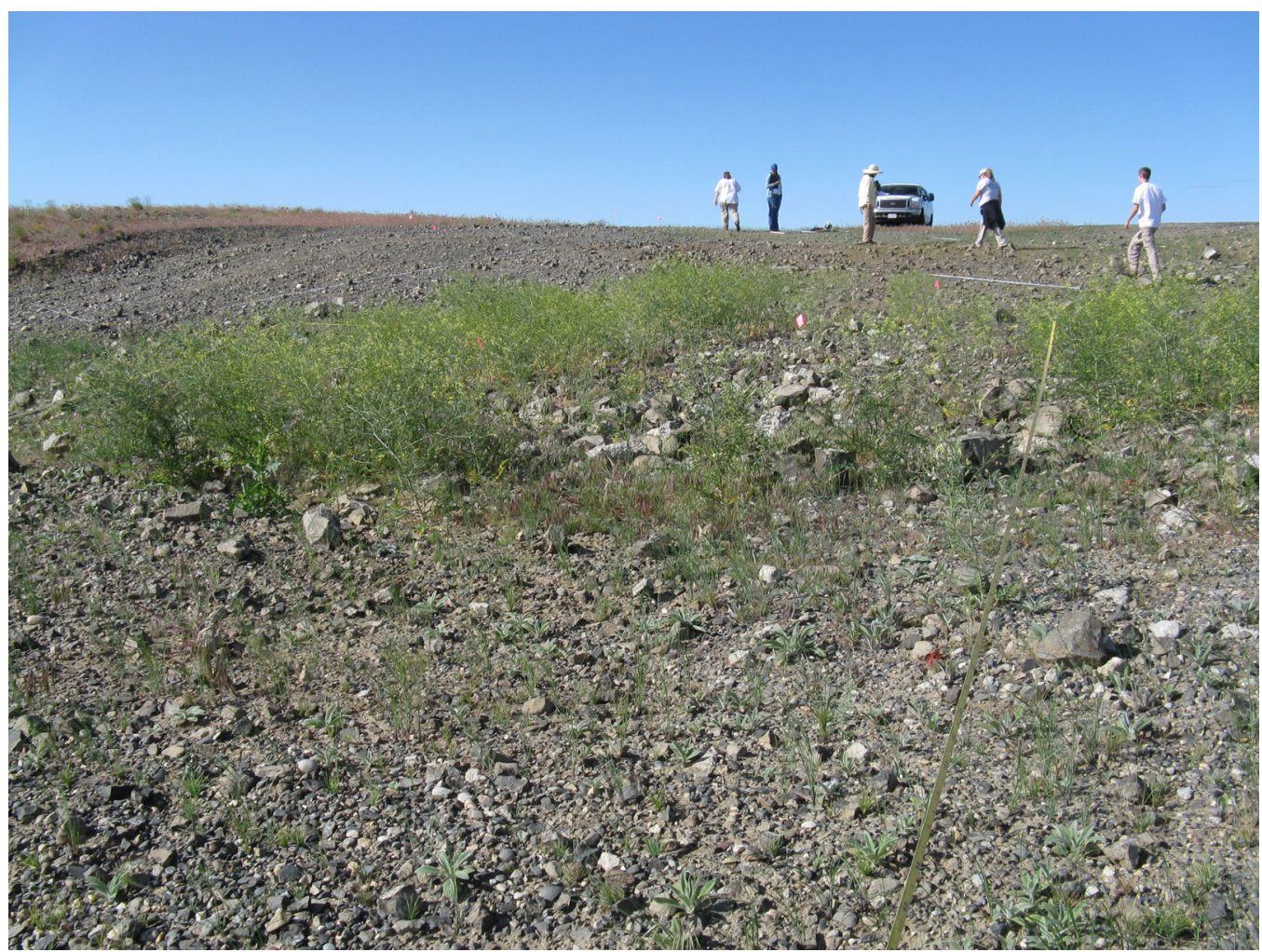

Figure B.21. Unit 4 (6652-C Bldgs): Plot 4-C-2, Starting Coordinates N 118429.54, E 569554.45

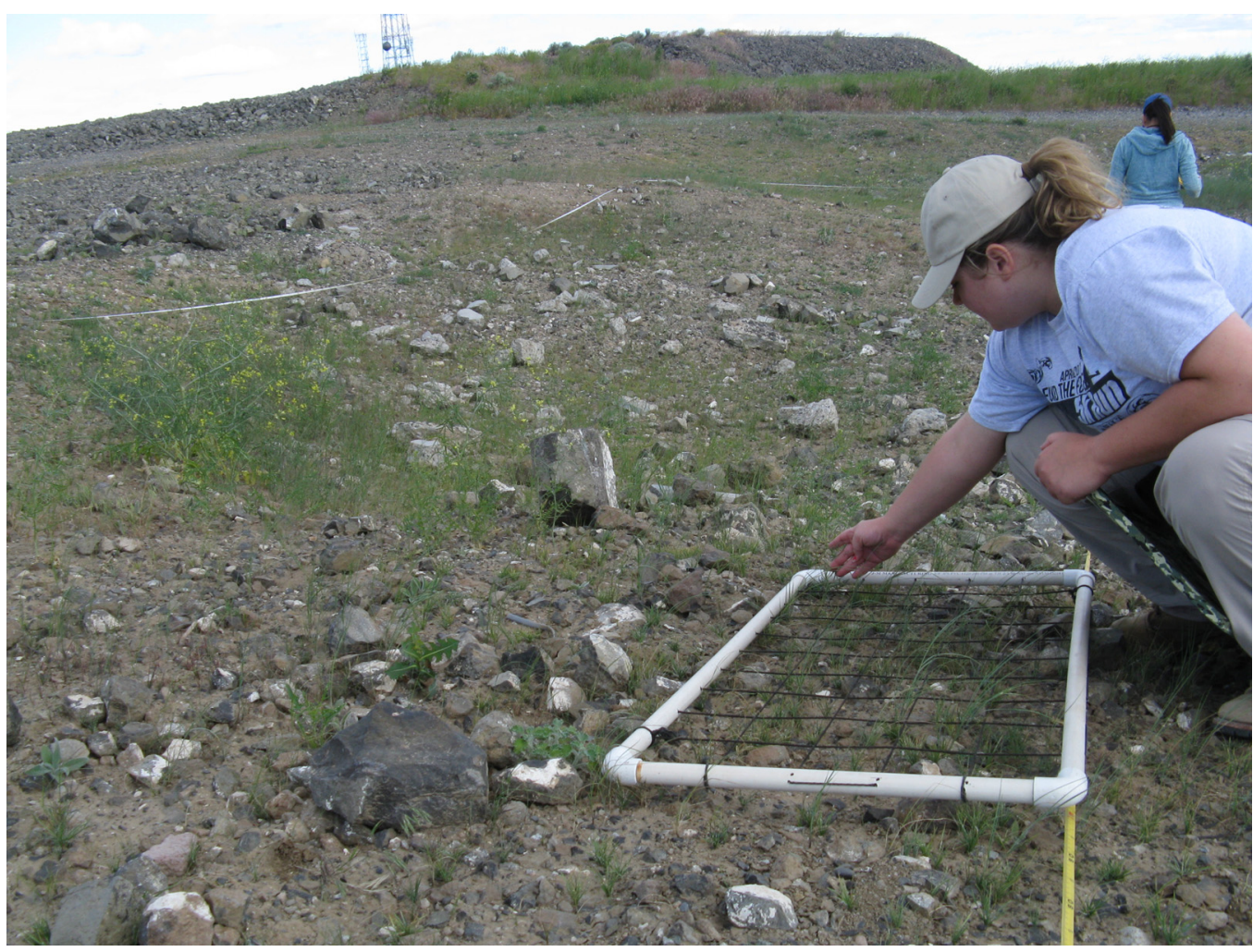

Figure B.22. Unit 4 (6652-C Bldgs): Plot 4-C-3, Starting Coordinates N 118404.54, E 569539.4 


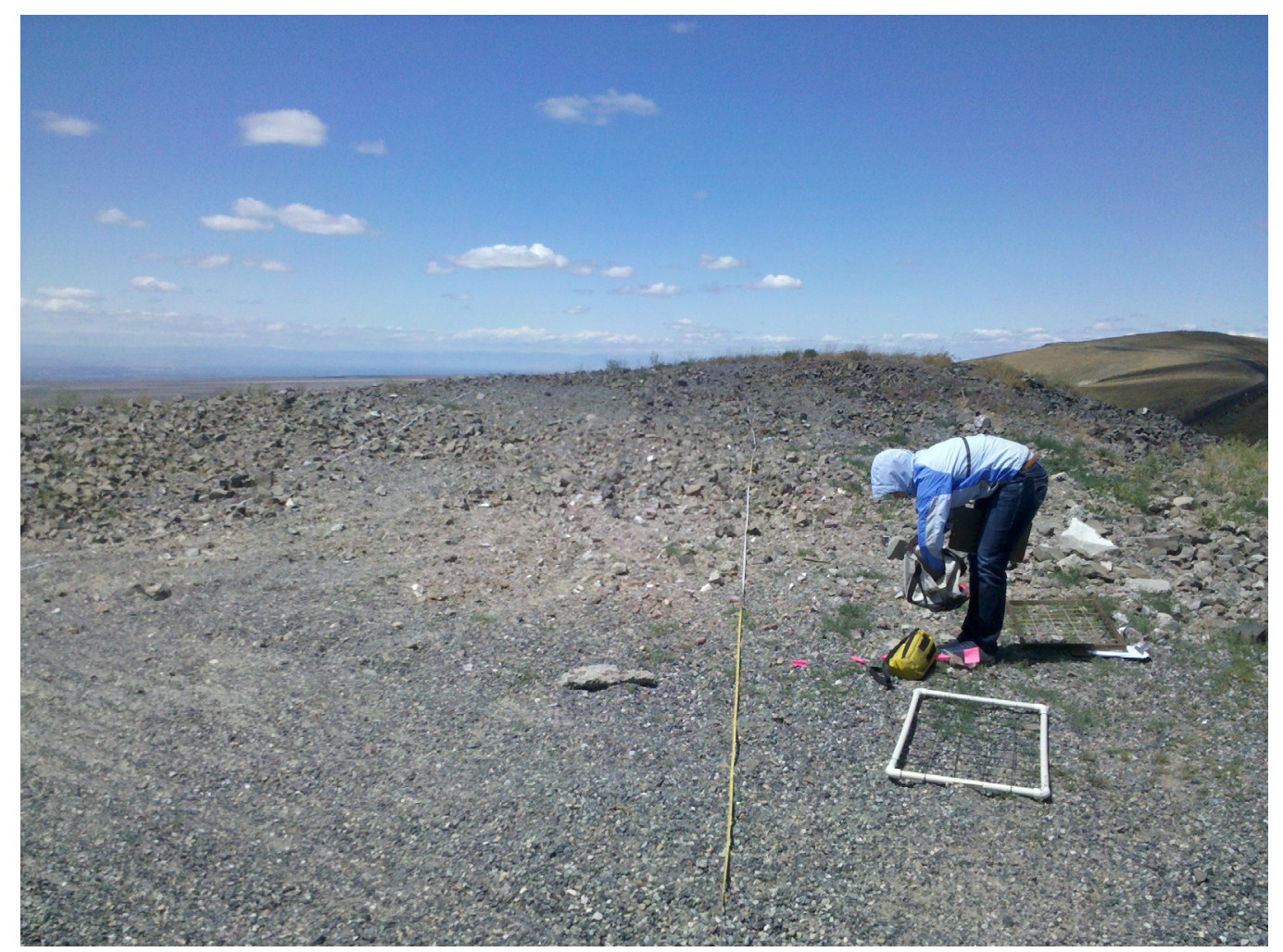

Figure B.23. Unit 4 (623A Bldg): Plot 4-623A-1, N 118007.498, E 570047.091

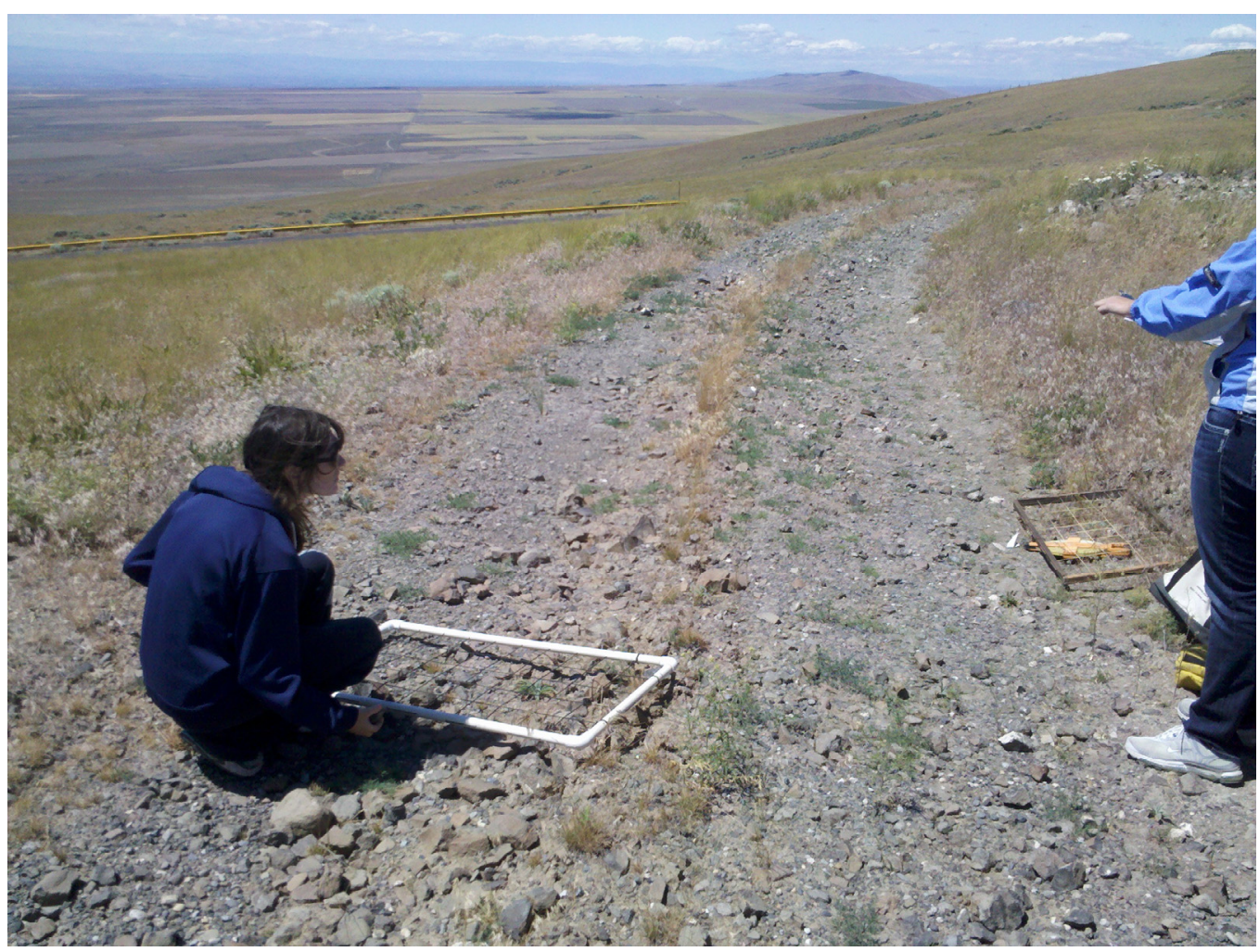

Figure B.24. Unit 4 (623A Road): Example of Road Surface Sampling 


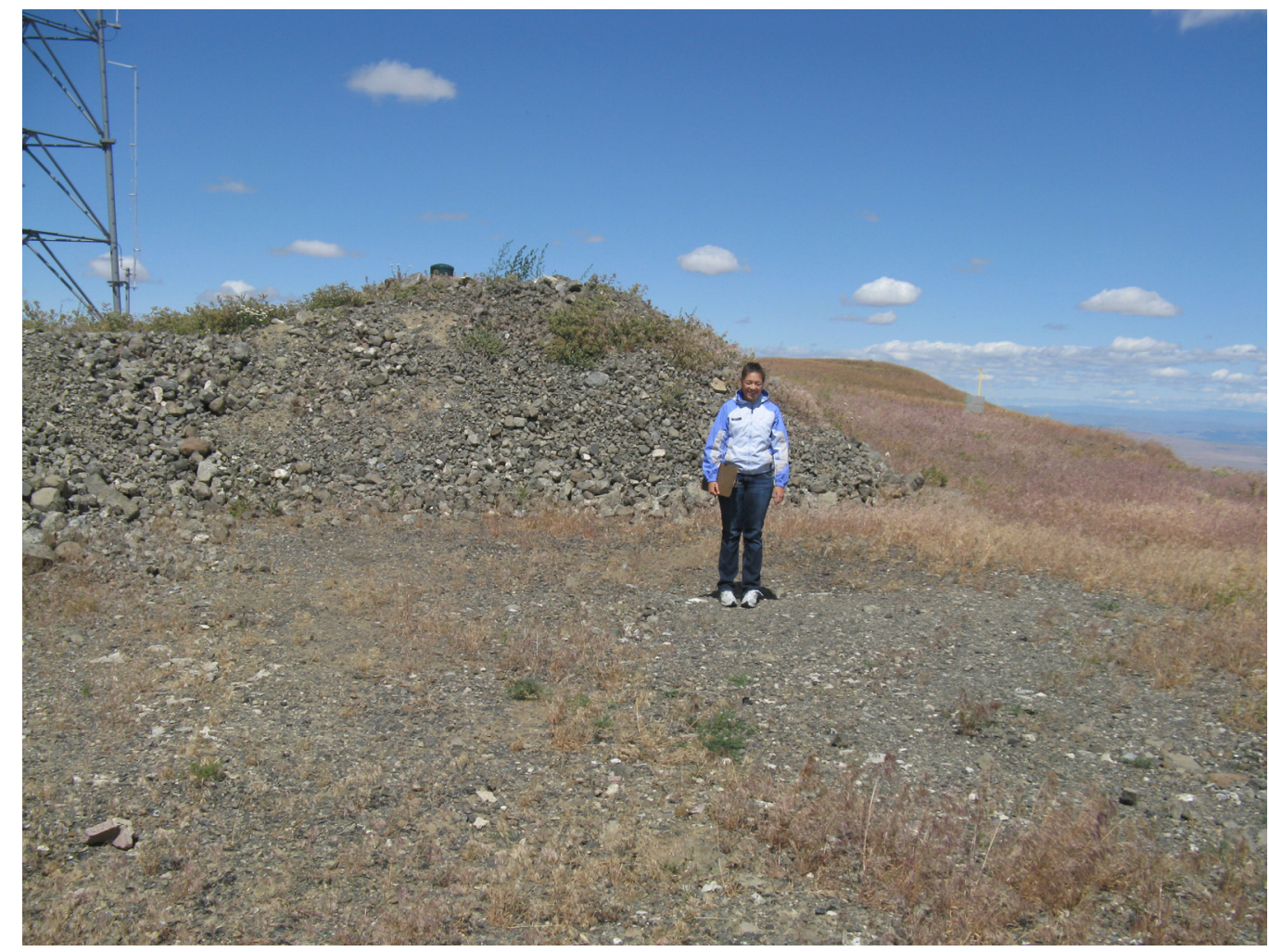

Figure B.25. Unit 4 (CCF Berms): Example of Berm Areas Sampled

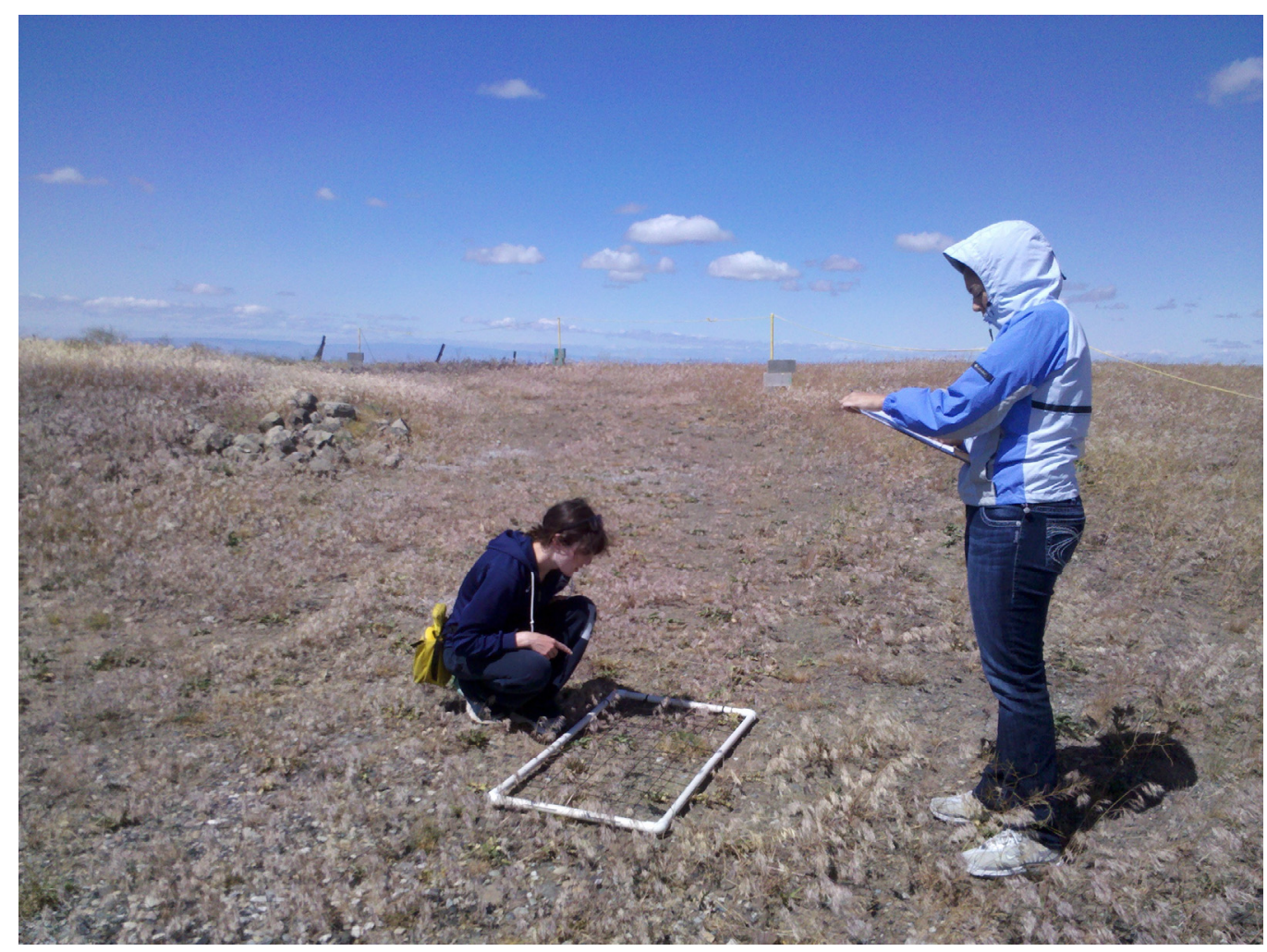

Figure B.26. Unit 4 (CCF): Example of Road Surface Sampling 


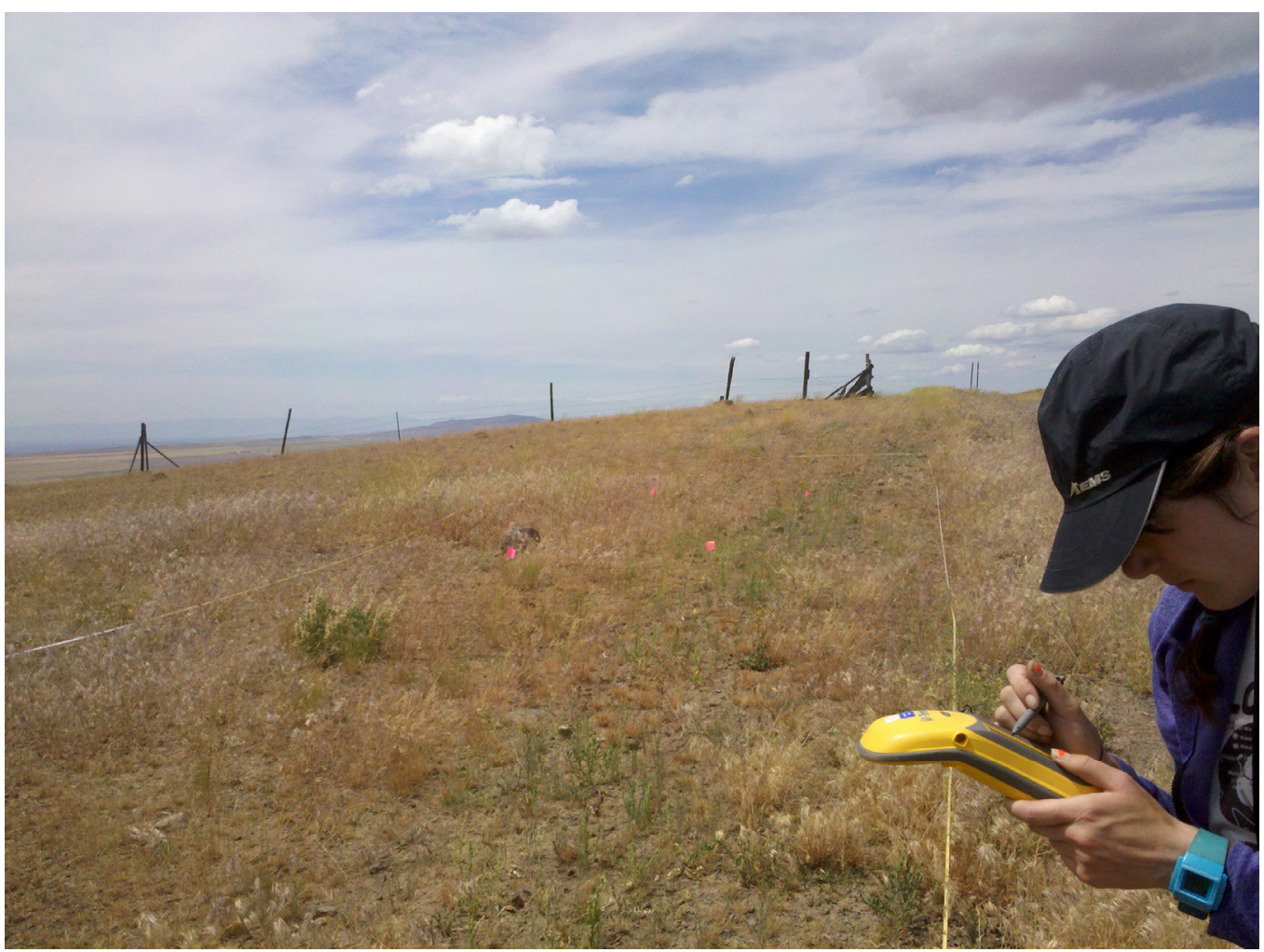

Figure B.27. Unit 4 (CCF): Plot4-CCF-1, Starting Coordinates N 118643.307, E 569239.409

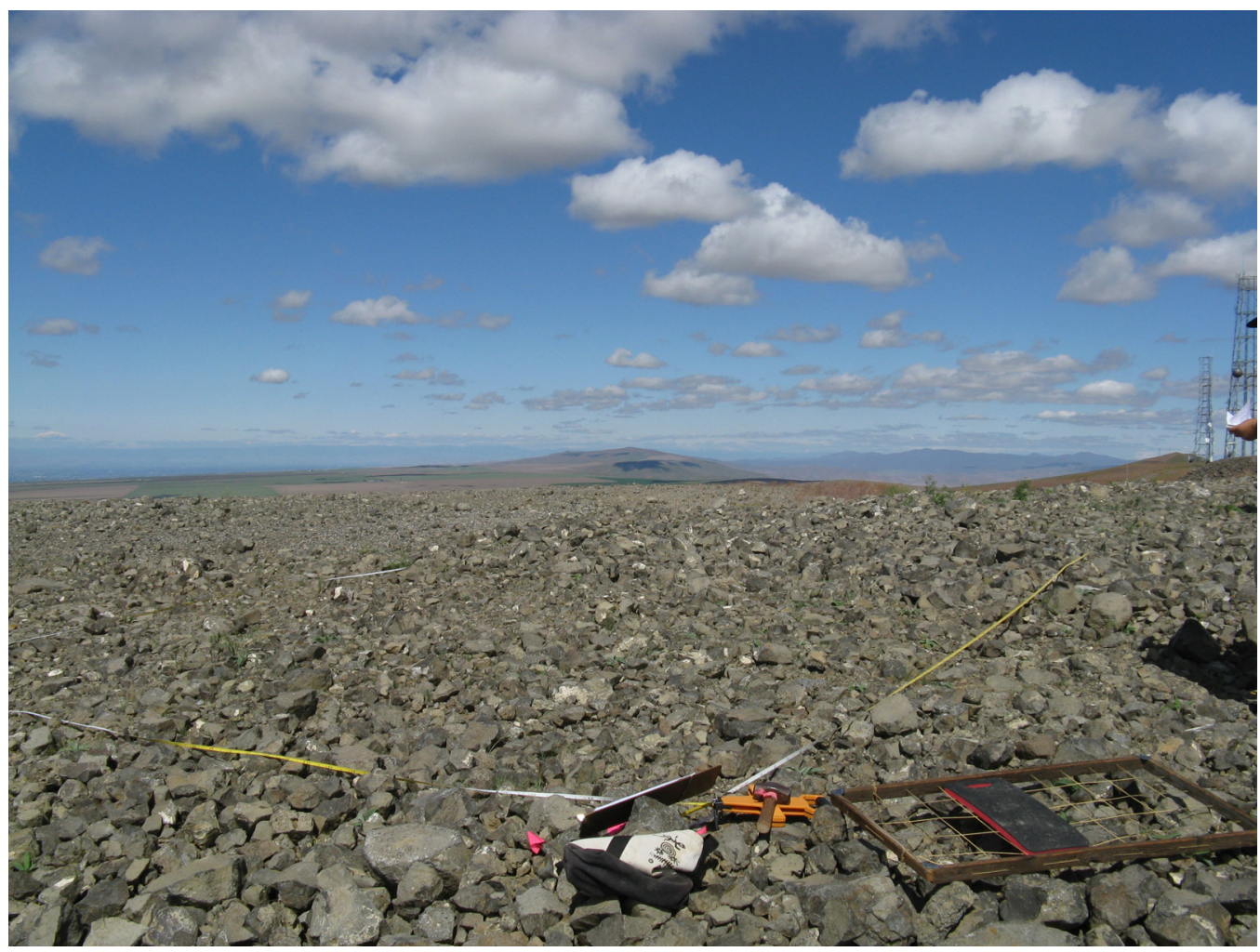

Figure B.28. Unit 5: Plot 5-OB-1, Starting Coordinates N 118428, E 569502.18 


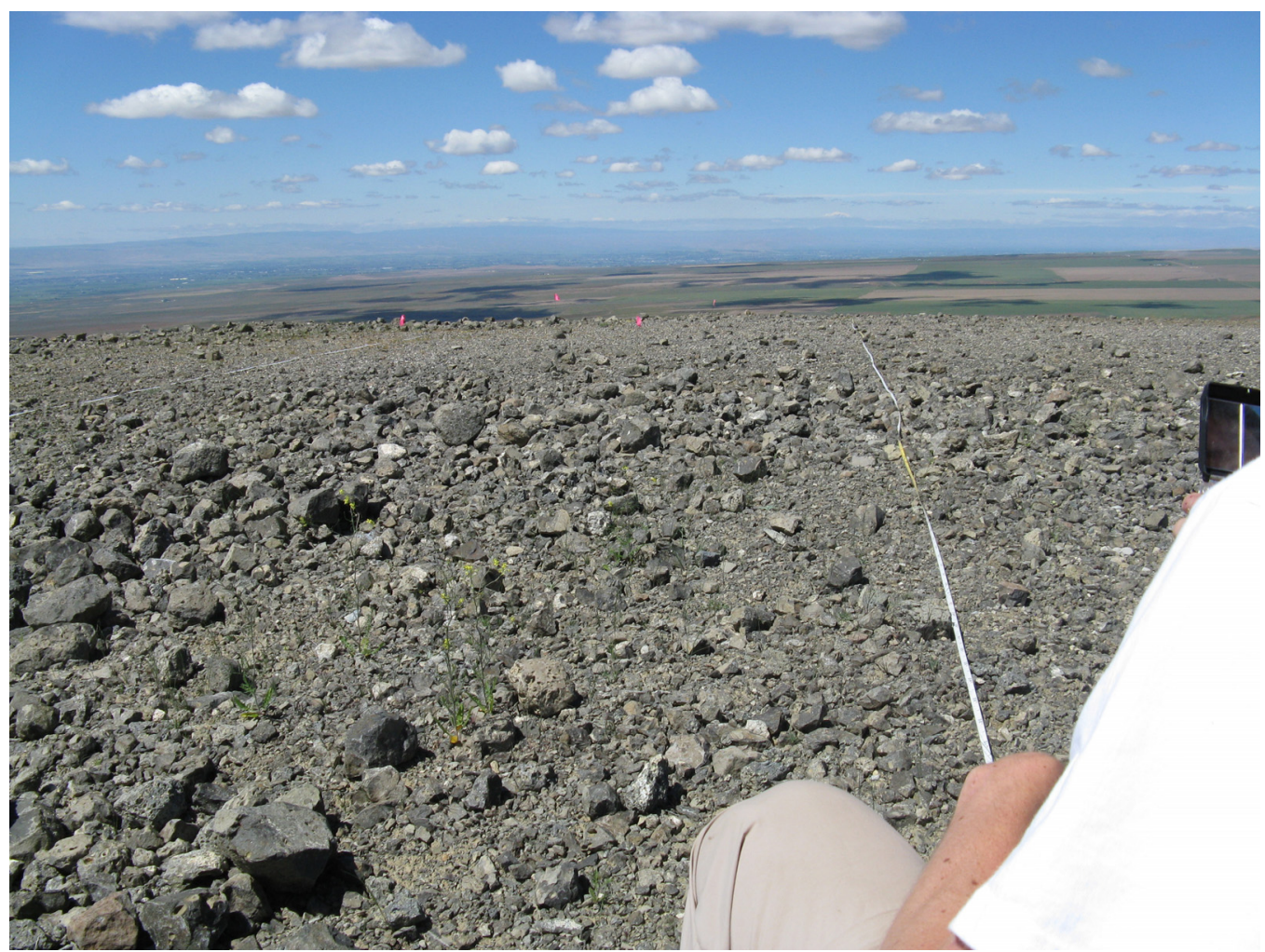

Figure B.29. Unit 5: Plot 5-OB-2, Starting Coordinate N 118426.38, E 569486.04

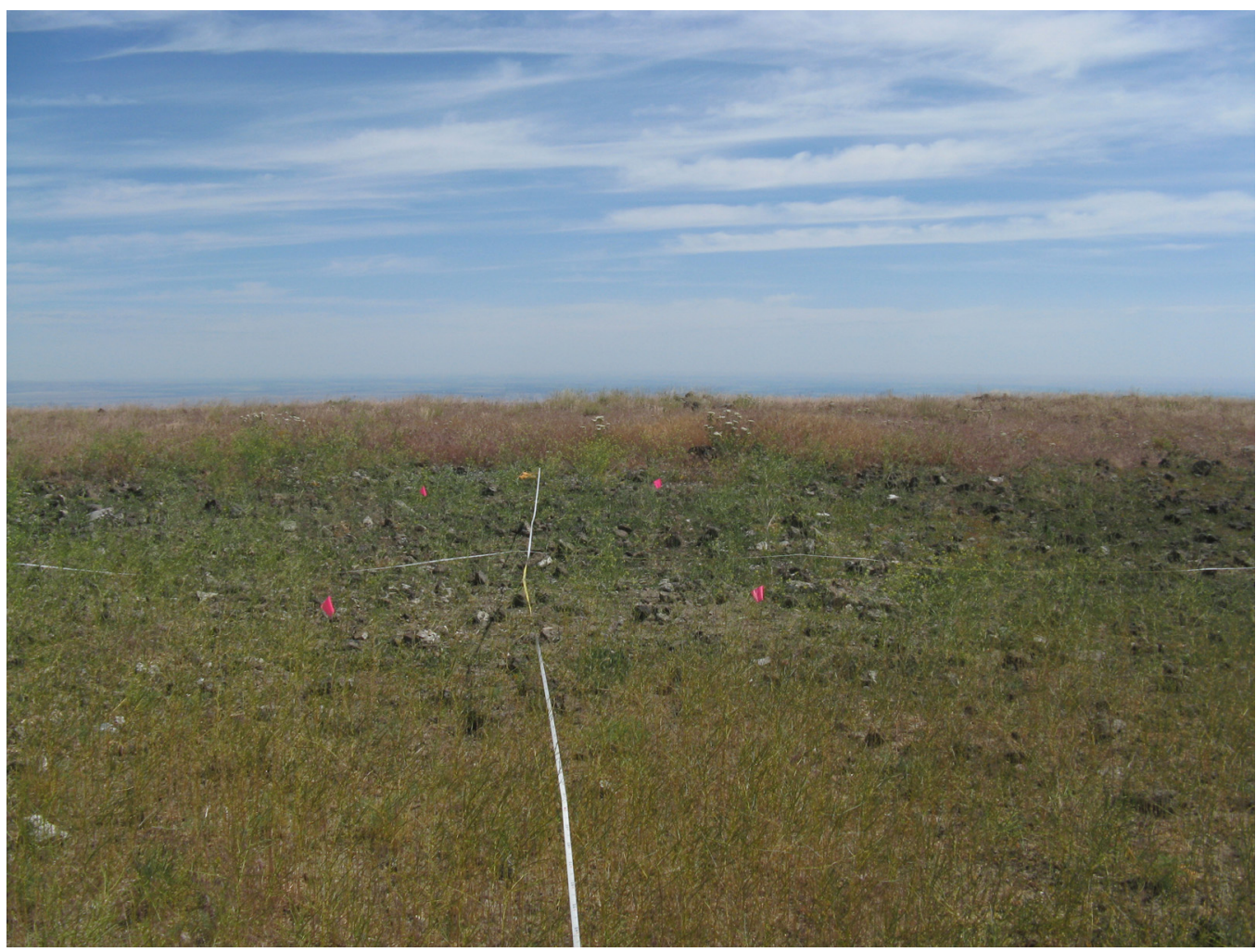

Figure B.30. Unit 6 (6635 Crown Castle): Plot 6-6635-X, Coordinates N 119117.54, E 568774.22 


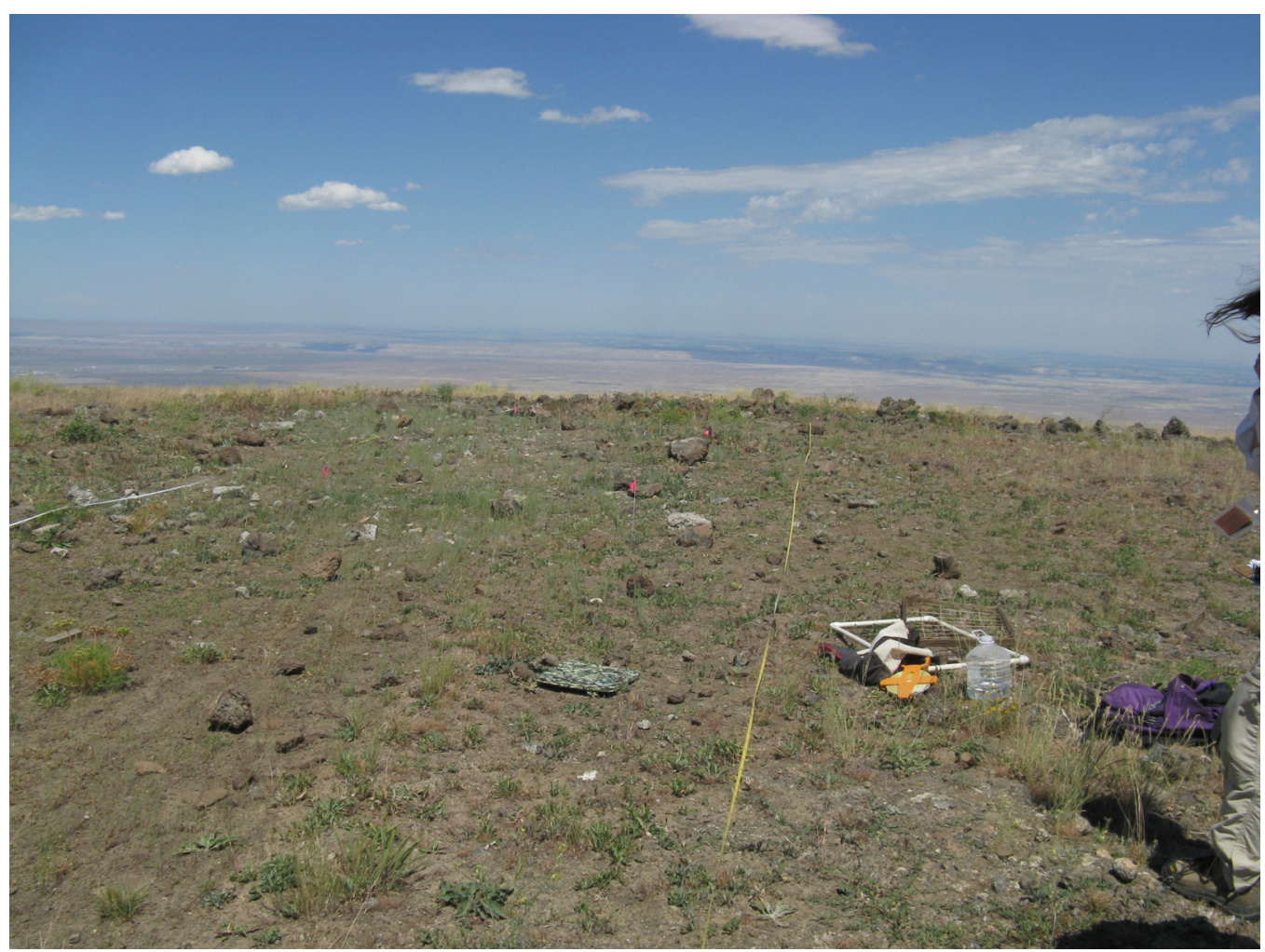

Figure B.31. Unit 6 (6636 Com Towers): Plot 6-6636-1, Coordinates N 119574.97, E 568365.6

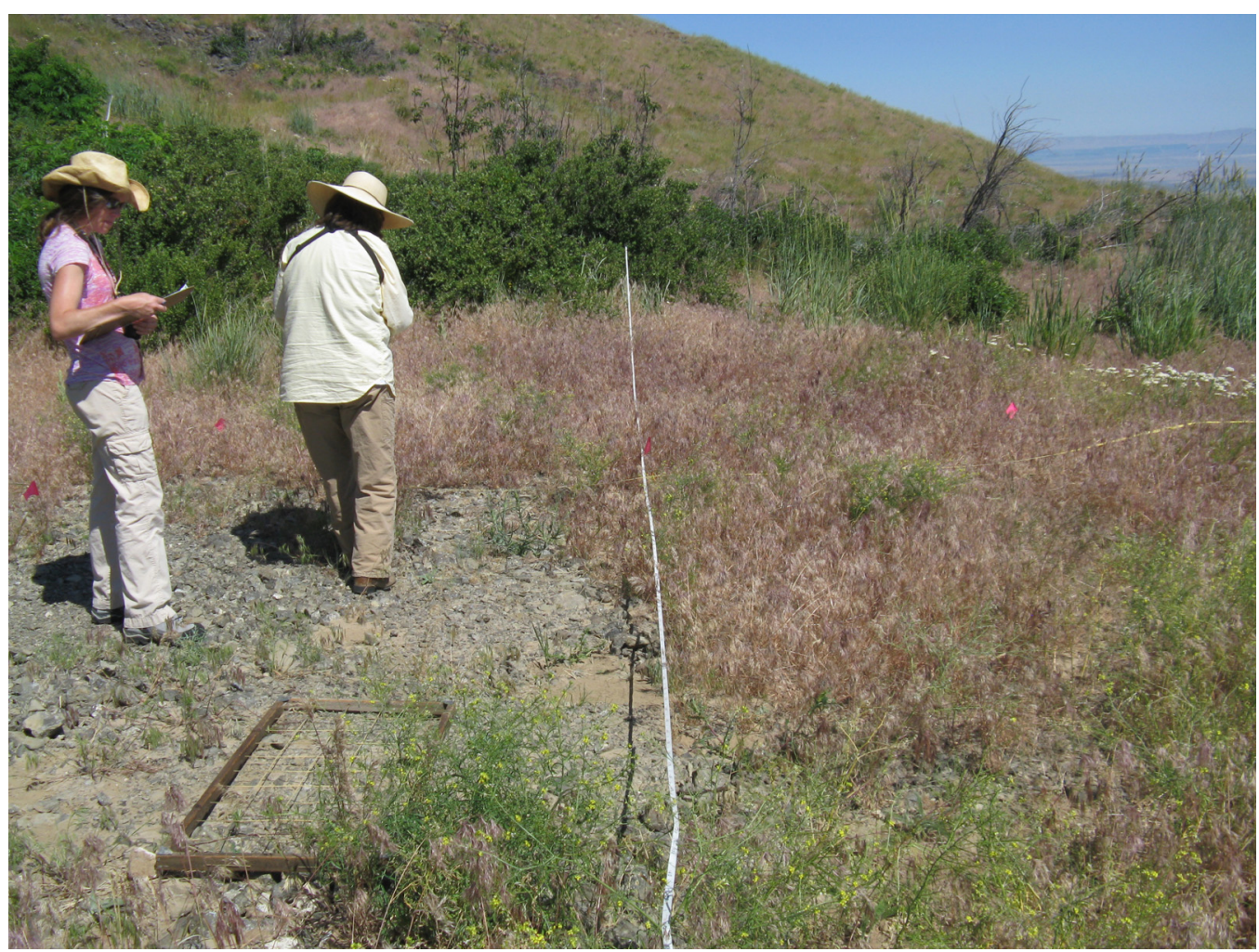

Figure B.32. Unit 7 (Pumphouse): Plot 7-PH-X, Coordinates N 119685.66, E 568699.77 


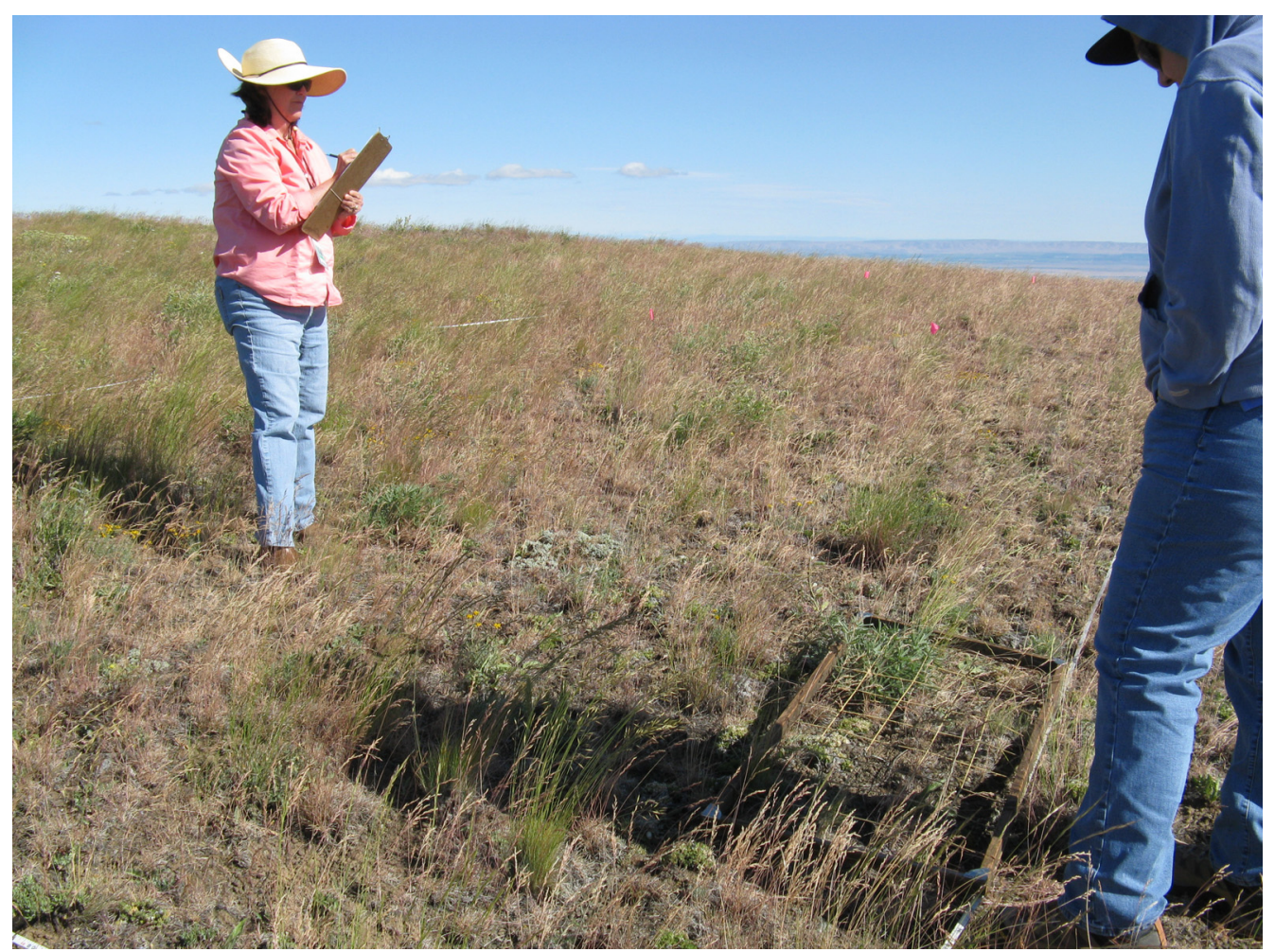

Figure B.33. Unit 0 (Ridgetop Ref): Plot RR-1, Starting Coordinates N 118791.649, E 569113.025

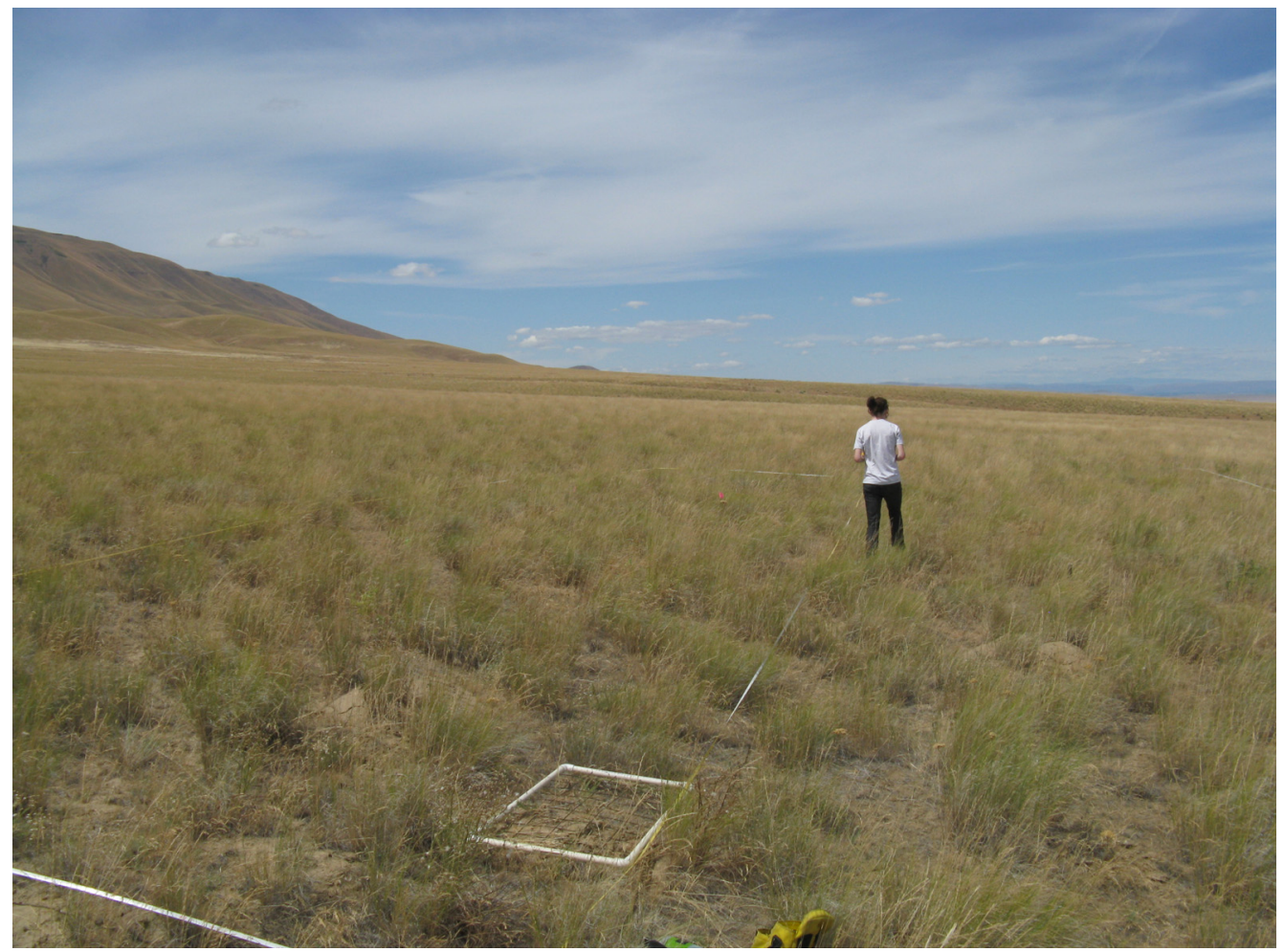

Figure B.34. Unit 0 (1200-ft Rd Ref): Plot 1200-REF-1, Coordinates N 118981.34, E 573325.59 


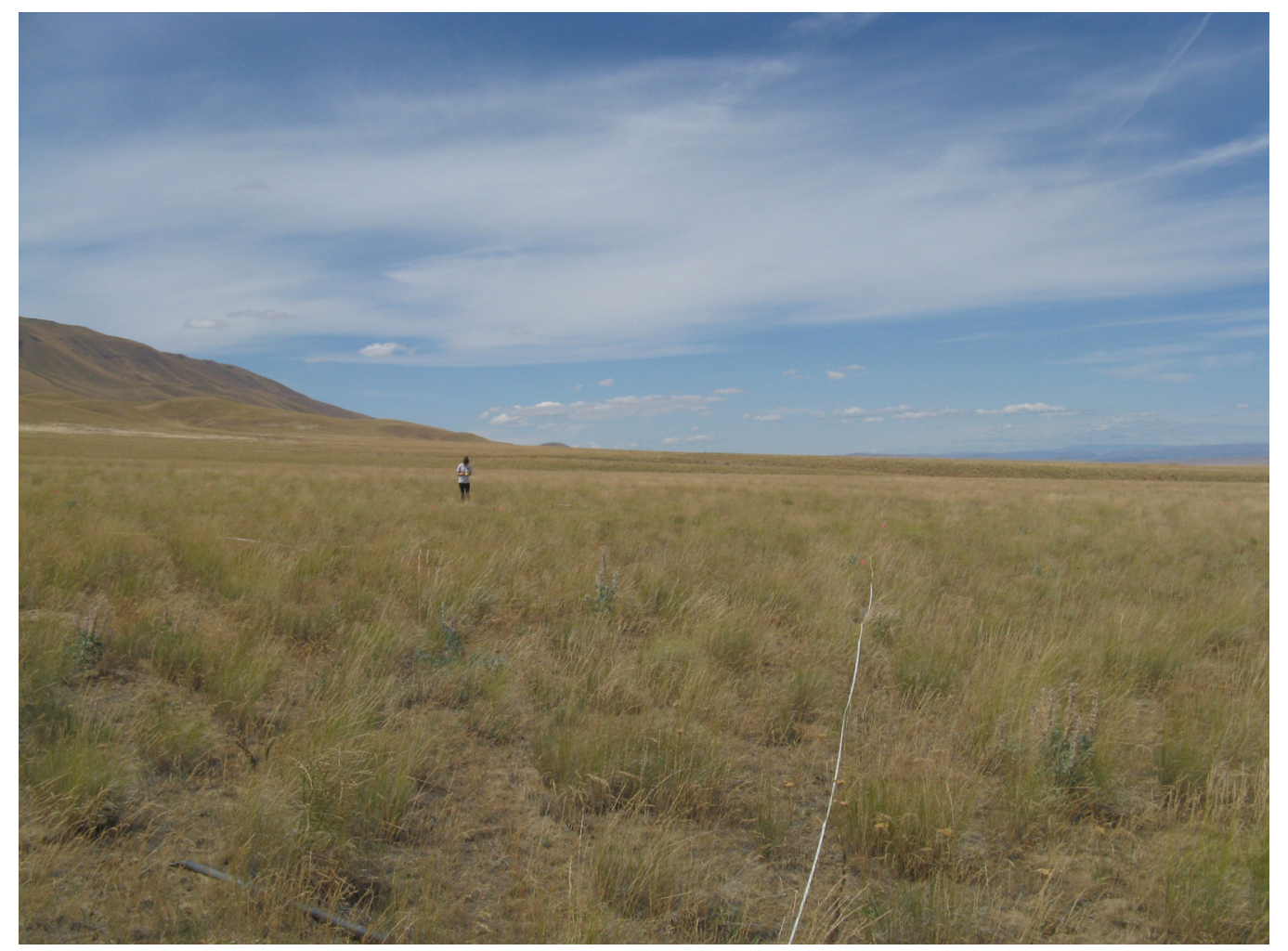

Figure B.35. Unit 0 (1200-ft Rd Ref): Photo of 20-m × 50-m Plot, N 118981.34, E 573325.59 
Appendix C

\section{Data Collected on Revegetation Monitoring Plots} on ALE During FY 2011 
Table C.1. Estimated Canopy Cover by Species Within 100- $\mathrm{m}^{2}$ Modified Whittaker Monitoring Plots

\begin{tabular}{|c|c|c|c|c|c|c|c|c|c|c|c|c|c|c|c|c|c|}
\hline $\begin{array}{c}\text { Reveg } \\
\text { Unit }\end{array}$ & Site Name & Genus/Species & Common Name & $1 \mathrm{~m}$ & $5 \mathrm{~m}$ & $\begin{array}{l}10 \\
\mathrm{~m}\end{array}$ & $\begin{array}{l}15 \\
\mathrm{~m}\end{array}$ & $\begin{array}{l}18 \\
\mathrm{~m}\end{array}$ & $\begin{array}{l}22 \\
\mathrm{~m}\end{array}$ & $\begin{array}{l}26 \\
\mathrm{~m}\end{array}$ & $\begin{array}{l}30 \\
\mathrm{~m}\end{array}$ & $\begin{array}{l}35 \\
\mathrm{~m}\end{array}$ & $\begin{array}{l}40 \\
\mathrm{~m}\end{array}$ & $\begin{array}{l}44 \\
\mathrm{~m}\end{array}$ & $\begin{array}{l}48 \\
\mathrm{~m}\end{array}$ & $\begin{array}{l}\text { Mean } \\
\text { Cover }\end{array}$ & Frequency \\
\hline 0 & 1200-REF-1 & Achillea millefolium & yarrow & 5 & 6 & 2 & 7 & 4 & 1 & 1 & 6 & & 1 & & & 2.75 & 0.75 \\
\hline 0 & 1200-REF-1 & Agropyron spicatum & bluebunch wheatgrass & 6 & 60 & 12 & 60 & 64 & 32 & 28 & 73 & 32 & 34 & 23 & 20 & 37.00 & 1.00 \\
\hline 0 & 1200-REF-1 & Astragalus purshii & woolly-pod milkvetch & & & 3 & & & & & & 1 & & & & 0.33 & 0.17 \\
\hline 0 & 1200-REF-1 & Bare Ground & Bare Ground & 72 & 42 & 30 & 11 & 11 & 15 & 27 & 5 & 5 & 9 & 43 & 38 & 25.67 & 1.00 \\
\hline 0 & 1200-REF-1 & Bromus tectorum & cheatgrass & 5 & 1 & & & & & & & & & & & 0.50 & 0.17 \\
\hline 0 & 1200-REF-1 & Calochortus macrocarpus & sagebrush mariposa lily & & & & & 1 & & & 1 & & & & & 0.17 & 0.17 \\
\hline 0 & 1200-REF-1 & Crepis atribarba & slender hawksbeard & & 1 & & 1 & 1 & & & & & 10 & & & 1.08 & 0.33 \\
\hline 0 & 1200-REF-1 & Cryptogarmic & Crust & 5 & 2 & 40 & 4 & 3 & 5 & 8 & 15 & 30 & 22 & 5 & 2 & 11.75 & 1.00 \\
\hline 0 & 1200-REF-1 & Erigeron filifolius & threadleaf fleabane & 7 & & 1 & 5 & & 2 & 7 & & & & 1 & 11 & 2.83 & 0.58 \\
\hline 0 & 1200-REF-1 & Holosteum umbellatum & jagged chickweed & & & & 4 & 5 & 2 & & & & & & & 0.92 & 0.25 \\
\hline 0 & 1200-REF-1 & Litter & Litter & 2 & 1 & 1 & 4 & 1 & 25 & 13 & 5 & 5 & 10 & 6 & 3 & 6.33 & 1.00 \\
\hline 0 & 1200-REF-1 & Lomatium macrocarpum & bigseed desertparsley & & 1 & 2 & & & & & 1 & & & 1 & 1 & 0.50 & 0.42 \\
\hline 0 & 1200-REF-1 & Lupinus sericeus & silky lupine & & & & 1 & & & & & & & & & 0.08 & 0.08 \\
\hline 0 & 1200-REF-1 & Phlox longifolia & longleaf phlox & & & & & & & 1 & & & 2 & & 1 & 0.33 & 0.25 \\
\hline 0 & 1200-REF-1 & Poa sandbergii & Sandberg's bluegrass & 48 & 15 & 14 & 7 & 5 & 20 & 16 & 17 & 28 & 12 & 21 & 25 & 19.00 & 1.00 \\
\hline 0 & RR-1 & Achillea millefolium & yarrow & 1 & & & & & 1 & & & 1 & 2 & & & 0.42 & 0.33 \\
\hline 0 & RR-1 & Agropyron spicatum & bluebunch wheatgrass & 6 & 4 & 6 & & 4 & & 2 & & 2 & & 15 & & 3.25 & 0.58 \\
\hline 0 & RR-1 & Antennaria dimorpha & low pussytoes & & & & & 1 & 1 & & & 1 & 3 & 1 & 1 & 0.67 & 0.50 \\
\hline 0 & RR-1 & Astragalus purshii & woolly-pod milkvetch & & 1 & 1 & 1 & 1 & & & 1 & & & & & 0.42 & 0.42 \\
\hline 0 & RR-1 & Balsamorhiza rosea & rosy balsamroot & 4 & 1 & 8 & 1 & 1 & 1 & 26 & 5 & 6 & 6 & 6 & 2 & 5.58 & 1.00 \\
\hline 0 & RR-1 & Bare Ground & Bare Ground & 27 & 48 & 46 & 58 & 56 & 52 & 30 & 56 & 47 & 45 & 4 & 38 & 42.25 & 1.00 \\
\hline 0 & RR-1 & Bromus tectorum & cheatgrass & & & & & & 1 & 1 & 1 & 1 & & & & 0.33 & 0.33 \\
\hline 0 & RR-1 & Crepis modocensis & low hawksbeard & 2 & 5 & 3 & & 3 & 4 & 1 & 2 & 4 & 6 & 5 & 5 & 3.33 & 0.92 \\
\hline 0 & RR-1 & Cryptogarmic & Crust & 18 & 12 & 14 & 7 & 7 & 12 & 8 & 7 & 8 & 8 & 2 & 16 & 9.92 & 1.00 \\
\hline 0 & RR-1 & Erigeron linearis & desert yellowdaisy & & & & & 1 & & & & & & 2 & & 0.25 & 0.17 \\
\hline 0 & RR-1 & Eriogonum sphaerocephalum & rock buckwheat & 1 & & & 4 & 4 & & 5 & & & & & 4 & 1.50 & 0.42 \\
\hline 0 & RR-1 & Eriogonum thymoides & thymeleaf buckwheat & & 5 & & 5 & 1 & 2 & 1 & & & & & & 1.17 & 0.42 \\
\hline 0 & RR-1 & Festuca idahoensis & Idaho fescue & 4 & & & & & 1 & & 4 & 4 & 7 & & 6 & 2.17 & 0.50 \\
\hline 0 & RR-1 & Haplopappus stenophylla & narrowleaf goldenweed & & & 1 & & 1 & & 1 & & & 1 & & & 0.33 & 0.33 \\
\hline 0 & RR-1 & Litter & Litter & 5 & 2 & 1 & 1 & 2 & 1 & 1 & 1 & 2 & 1 & 4 & 1 & 1.83 & 1.00 \\
\hline
\end{tabular}


Table C.1. (contd)

\begin{tabular}{|c|c|c|c|c|c|c|c|c|c|c|c|c|c|c|c|c|c|}
\hline $\begin{array}{c}\text { Reveg } \\
\text { Unit }\end{array}$ & Site Name & Genus/Species & Common Name & $1 \mathrm{~m}$ & $5 \mathrm{~m}$ & $\begin{array}{l}10 \\
\mathrm{~m}\end{array}$ & $\begin{array}{l}15 \\
\mathrm{~m}\end{array}$ & $\begin{array}{l}18 \\
\mathrm{~m}\end{array}$ & $\begin{array}{l}22 \\
\mathrm{~m}\end{array}$ & $\begin{array}{l}26 \\
\mathrm{~m}\end{array}$ & $\begin{array}{l}30 \\
\mathrm{~m}\end{array}$ & $\begin{array}{l}35 \\
\mathrm{~m}\end{array}$ & $\begin{array}{l}40 \\
\mathrm{~m}\end{array}$ & $\begin{array}{l}44 \\
\mathrm{~m}\end{array}$ & $\begin{array}{l}48 \\
\mathrm{~m} \\
\end{array}$ & $\begin{array}{l}\text { Mean } \\
\text { Cover }\end{array}$ & Frequency \\
\hline 0 & RR-1 & Lupinus sericeus & silky lupine & 14 & & & & & & & & 1 & 4 & 50 & 2 & 5.92 & 0.42 \\
\hline 0 & RR-1 & Phlox hoodii & Hood's phlox & 7 & 8 & 4 & 7 & 3 & 8 & 6 & 8 & 3 & 2 & 5 & 2 & 5.25 & 1.00 \\
\hline 0 & RR-1 & Phoenicaulis cheiranthoides & daggerpod & & & & & & & & 1 & & & & & 0.08 & 0.08 \\
\hline 0 & RR-1 & Poa sandbergii & Sandberg's bluegrass & 16 & 16 & 15 & 17 & 17 & 17 & 19 & 15 & 22 & 16 & 10 & 24 & 17.00 & 1.00 \\
\hline 0 & RR-1 & Sitanion hystrix & bottlebrush grass & & & 2 & & & & & & & & & & 0.17 & 0.08 \\
\hline 1 & 1-RS-1 & Bare Ground & Bare Ground & 81 & 90 & 26 & 74 & 60 & 50 & 65 & 90 & 60 & 65 & 85 & 75 & 68.42 & 1.00 \\
\hline 1 & 1-RS-1 & Kochia scoparia & summer cypress & & & 1 & & & & & & & & & & 0.08 & 0.08 \\
\hline 1 & 1-RS-1 & Litter & Litter & 9 & 10 & 65 & 26 & 16 & 38 & 34 & 8 & 32 & 32 & 22 & 20 & 26.00 & 1.00 \\
\hline 1 & 1-RS-1 & Salsola kali & Russian thistle & & 1 & 14 & 2 & 8 & 4 & 3 & 6 & 7 & 2 & & 1 & 4.00 & 0.83 \\
\hline 1 & 1-RS-1 & Sitanion hystrix & bottlebrush grass & & & 4 & & & & & 2 & 5 & & & & 0.92 & 0.25 \\
\hline 1 & 1-RS-2 & Artemisia tridentata & big sagebrush & & & & & & & & & 1 & & & & 0.08 & 0.08 \\
\hline 1 & 1-RS-2 & Bare Ground & Bare Ground & 80 & 18 & 50 & 28 & 75 & 5 & 35 & 20 & 18 & 30 & 34 & 50 & 36.92 & 1.00 \\
\hline 1 & 1-RS-2 & Bromus tectorum & cheatgrass & & 8 & & & & & & & & & & & 0.67 & 0.08 \\
\hline 1 & 1-RS-2 & Distichlis stricta & alkali saltgrass & 4 & 40 & 16 & 2 & & 1 & 16 & 20 & 2 & & & & 8.42 & 0.67 \\
\hline 1 & 1-RS-2 & Elymus cinereus & giant wildrye & & & & 4 & & & & & & & & & 0.33 & 0.08 \\
\hline 1 & 1-RS-2 & Litter & Litter & 10 & 8 & 24 & 8 & 18 & 95 & 14 & 26 & 16 & 6 & 8 & 22 & 21.25 & 1.00 \\
\hline 1 & 1-RS-2 & Salsola kali & Russian thistle & 2 & 4 & 2 & 30 & 2 & 7 & 22 & 5 & 22 & 59 & 66 & 10 & 19.25 & 1.00 \\
\hline 1 & 1-RS-2 & Sitanion hystrix & bottlebrush grass & 2 & & 2 & 2 & & & & 4 & 6 & 1 & 1 & 2 & 1.67 & 0.67 \\
\hline 1 & 1-RS-3 & Achillea millefolium & yarrow & & & & & & & & & 2 & & & & 0.17 & 0.08 \\
\hline 1 & 1-RS-3 & Artemisia tridentata & big sagebrush & & 1 & & & & & & & & & & & 0.08 & 0.08 \\
\hline 1 & 1-RS-3 & Bare Ground & Bare Ground & 63 & 58 & 56 & 60 & 70 & 75 & 65 & 60 & 50 & 75 & 70 & 45 & 62.25 & 1.00 \\
\hline 1 & 1-RS-3 & Bromus tectorum & cheatgrass & & & & & & 4 & 1 & & 8 & 2 & 8 & 5 & 2.33 & 0.50 \\
\hline 1 & $1-\mathrm{RS}-3$ & Distichlis stricta & alkali saltgrass & 2 & & 2 & & & & 10 & & & & 1 & & 1.25 & 0.33 \\
\hline 1 & 1-RS-3 & Elymus cinereus & giant wildrye & & & & & 1 & 1 & & & 1 & & & & 0.25 & 0.25 \\
\hline 1 & 1-RS-3 & Grayia spinosa & spiny hopsage & & & & & & & & & 1 & & & & 0.08 & 0.08 \\
\hline 1 & $1-\mathrm{RS}-3$ & Kochia scoparia & summer cypress & & 1 & & & & & & & & & & & 0.08 & 0.08 \\
\hline 1 & 1-RS-3 & Litter & Litter & 31 & 26 & 30 & 33 & 14 & 10 & 15 & 31 & 14 & 15 & 9 & 27 & 21.25 & 1.00 \\
\hline 1 & $1-\mathrm{RS}-3$ & Machaeranthera canescens & hoary aster & & & & & & & & & & 2 & & & 0.17 & 0.08 \\
\hline 1 & $1-\mathrm{RS}-3$ & Poa sandbergii & Sandberg's bluegrass & & & & & & 1 & & & & 1 & 2 & 2 & 0.50 & 0.33 \\
\hline 1 & 1-RS-3 & Salsola kali & Russian thistle & 8 & 7 & 5 & 6 & 4 & 1 & 4 & 16 & 10 & 2 & 7 & 4 & 6.17 & 1.00 \\
\hline
\end{tabular}


Table C.1. (contd)

\begin{tabular}{|c|c|c|c|c|c|c|c|c|c|c|c|c|c|c|c|c|c|}
\hline $\begin{array}{l}\text { Reveg } \\
\text { Unit }\end{array}$ & Site Name & Genus/Species & Common Name & $1 \mathrm{~m}$ & $5 \mathrm{~m}$ & $\begin{array}{l}10 \\
\mathrm{~m}\end{array}$ & $\begin{array}{l}15 \\
\mathrm{~m}\end{array}$ & $\begin{array}{l}18 \\
\mathrm{~m}\end{array}$ & $\begin{array}{c}22 \\
\mathrm{~m}\end{array}$ & $\begin{array}{c}26 \\
\mathrm{~m}\end{array}$ & $\begin{array}{l}30 \\
\mathrm{~m}\end{array}$ & $\begin{array}{l}35 \\
\mathrm{~m}\end{array}$ & $\begin{array}{l}40 \\
\mathrm{~m}\end{array}$ & $\begin{array}{l}44 \\
\mathrm{~m}\end{array}$ & $\begin{array}{l}48 \\
\mathrm{~m}\end{array}$ & $\begin{array}{l}\text { Mean } \\
\text { Cover }\end{array}$ & Frequency \\
\hline 1 & 1-RS-3 & Sisymbrium altissimum & $\begin{array}{l}\text { Jim Hill's } \\
\text { tumblemustard }\end{array}$ & & & & & 1 & 1 & & 1 & 2 & & 4 & 1 & 0.83 & 0.50 \\
\hline 1 & 1-RS-3 & Sitanion hystrix & bottlebrush grass & 3 & 2 & 2 & 5 & 3 & 2 & 1 & 1 & 1 & 3 & 3 & 4 & 2.50 & 1.00 \\
\hline 2 & $2-109-1$ & Achillea millefolium & yarrow & & & & & & & & & & & 2 & 2 & 0.33 & 0.17 \\
\hline 2 & $2-109-1$ & Agropyron spicatum & bluebunch wheatgrass & 1 & 2 & 2 & & & & & 1 & 2 & & & 2 & 0.83 & 0.50 \\
\hline 2 & $2-109-1$ & Amsinckia tessellata & devil's lettuce & 7 & 1 & & & & & & & 10 & 8 & & & 2.17 & 0.33 \\
\hline 2 & $2-109-1$ & Bare Ground & Bare Ground & 40 & 70 & 30 & 21 & 4 & 1 & 3 & & 70 & 4 & & 10 & 21.08 & 0.83 \\
\hline 2 & $2-109-1$ & Bromus tectorum & cheatgrass & 20 & 12 & 18 & 35 & 40 & 15 & 25 & 50 & 2 & 22 & 45 & 15 & 24.92 & 1.00 \\
\hline 2 & $2-109-1$ & Crepis atribarba & slender hawksbeard & & & & 7 & & 8 & & 2 & 2 & & & & 1.58 & 0.33 \\
\hline 2 & $2-109-1$ & Erodium cicutarium & storksbill & & & & & & 1 & & & & 1 & 1 & & 0.25 & 0.25 \\
\hline 2 & $2-109-1$ & Lactuca serriola & prickly lettuce & & & 2 & 1 & & 1 & & 2 & 4 & 5 & & 2 & 1.42 & 0.58 \\
\hline 2 & $2-109-1$ & Linum perenne & wild blueflax & & & & & & & 3 & & & & & & 0.25 & 0.08 \\
\hline 2 & 2-109-1 & Litter & Litter & 50 & 15 & 45 & 74 & 65 & 75 & 80 & 60 & 4 & 60 & 60 & 50 & 53.17 & 1.00 \\
\hline 2 & $2-109-1$ & Lupinus sericeus & silky lupine & 1 & 13 & 12 & 19 & 5 & 2 & 16 & 28 & 2 & 2 & & 12 & 9.33 & 0.92 \\
\hline 2 & $2-109-1$ & Machaeranthera canescens & hoary aster & & & & 1 & & & & & & & & & 0.08 & 0.08 \\
\hline 2 & $2-109-1$ & Poa bulbosa & bulbous bluegrass & & 2 & 1 & 2 & 2 & 4 & 2 & 4 & & 1 & 1 & 1 & 1.67 & 0.83 \\
\hline 2 & $2-109-1$ & Poa sandbergii & Sandberg's bluegrass & 1 & & & & & & & 1 & 2 & & & 1 & 0.42 & 0.33 \\
\hline 2 & $2-109-1$ & Sisymbrium altissimum & $\begin{array}{l}\text { Jim Hill's } \\
\text { tumblemustard }\end{array}$ & 6 & 2 & 7 & & 30 & 1 & & & 20 & & 4 & & 5.83 & 0.58 \\
\hline 2 & $2-109-1$ & Sitanion hystrix & bottlebrush grass & & & & 1 & & & & & & & & & 0.08 & 0.08 \\
\hline 2 & $2-109-1$ & Stipa thurberiana & Thurber's needlegrass & 2 & 15 & 8 & 1 & & 9 & 14 & & & 8 & 1 & 12 & 5.83 & 0.75 \\
\hline 2 & $2-109-2$ & Agropyron spicatum & bluebunch wheatgrass & 14 & 10 & 8 & 22 & 32 & 30 & 11 & & 45 & 17 & 18 & 44 & 20.92 & 0.92 \\
\hline 2 & $2-109-2$ & Astragalus caricinus & buckwheat milkvetch & 1 & 33 & 20 & 20 & 13 & 11 & 10 & 5 & 8 & 14 & & & 11.25 & 0.83 \\
\hline 2 & $2-109-2$ & Bare Ground & Bare Ground & 10 & 45 & 50 & 15 & 10 & 9 & 38 & 65 & 14 & 75 & 34 & 30 & 32.92 & 1.00 \\
\hline 2 & $2-109-2$ & Bromus tectorum & cheatgrass & 6 & 2 & 2 & 8 & 10 & 6 & 10 & 18 & 3 & 1 & 1 & 1 & 5.67 & 1.00 \\
\hline 2 & $2-109-2$ & Crepis atribarba & slender hawksbeard & 4 & & 16 & 2 & 4 & 2 & & & 2 & 1 & 2 & 10 & 3.58 & 0.75 \\
\hline 2 & $2-109-2$ & Erigeron filifolius & threadleaf fleabane & & & & & 5 & & & & & & & & 0.42 & 0.08 \\
\hline 2 & $2-109-2$ & Litter & Litter & 55 & 35 & 32 & 61 & 28 & 55 & 35 & 18 & 20 & 8 & 50 & 18 & 34.58 & 1.00 \\
\hline 2 & $2-109-2$ & Lupinus sericeus & silky lupine & & & & 2 & & & & & & & 12 & & 1.17 & 0.17 \\
\hline 2 & $2-109-2$ & Phlox longifolia & longleaf phlox & 1 & 4 & 4 & & & 4 & 6 & 18 & 1 & 3 & 7 & 6 & 4.50 & 0.83 \\
\hline 2 & $2-109-2$ & Poa bulbosa & bulbous bluegrass & 2 & 1 & 1 & 2 & 6 & 2 & 2 & 10 & & 1 & 2 & 1 & 2.50 & 0.92 \\
\hline 2 & $2-109-2$ & Poa sandbergii & Sandberg's bluegrass & 2 & & 1 & 1 & 5 & 5 & 2 & 1 & 2 & 2 & 1 & 3 & 2.08 & 0.92 \\
\hline
\end{tabular}


Table C.1. (contd)

\begin{tabular}{|c|c|c|c|c|c|c|c|c|c|c|c|c|c|c|c|c|c|}
\hline $\begin{array}{l}\text { Reveg } \\
\text { Unit }\end{array}$ & Site Name & Genus/Species & Common Name & $1 \mathrm{~m}$ & $5 \mathrm{~m}$ & $\begin{array}{l}10 \\
\mathrm{~m}\end{array}$ & $\begin{array}{l}15 \\
\mathrm{~m}\end{array}$ & $\begin{array}{l}18 \\
\mathrm{~m}\end{array}$ & $\begin{array}{l}22 \\
\mathrm{~m}\end{array}$ & $\begin{array}{l}26 \\
\mathrm{~m}\end{array}$ & $\begin{array}{l}30 \\
\mathrm{~m}\end{array}$ & $\begin{array}{l}35 \\
\mathrm{~m}\end{array}$ & $\begin{array}{l}40 \\
\mathrm{~m}\end{array}$ & $\begin{array}{l}44 \\
\mathrm{~m}\end{array}$ & $\begin{array}{l}48 \\
\mathrm{~m}\end{array}$ & $\begin{array}{l}\text { Mean } \\
\text { Cover }\end{array}$ & Frequency \\
\hline 2 & $2-109-2$ & Stipa comata & needle-and-thread grass & 38 & & 1 & 2 & 4 & 10 & & 18 & & & 2 & 8 & 6.92 & 0.67 \\
\hline 2 & $2-109-2$ & Tragopogon dubius & Yellow salsify & & & & 2 & & & & & & & & & 0.17 & 0.08 \\
\hline 2 & $2-109-3$ & Achillea millefolium & yarrow & 1 & 1 & 4 & & & 1 & 12 & 3 & 2 & & & & 2.00 & 0.58 \\
\hline 2 & $2-109-3$ & Agropyron spicatum & bluebunch wheatgrass & 18 & 15 & 20 & 2 & 16 & 14 & 28 & 30 & 35 & 9 & 6 & 14 & 17.25 & 1.00 \\
\hline 2 & $2-109-3$ & Artemisia tridentata & big sagebrush & & & & & & 1 & & & & & & & 0.08 & 0.08 \\
\hline 2 & $2-109-3$ & Astragalus caricinus & buckwheat milkvetch & & & & & & & 6 & & 16 & 5 & & & 2.25 & 0.25 \\
\hline 2 & $2-109-3$ & Bare Ground & Bare Ground & 60 & 80 & 60 & 70 & 50 & 40 & 40 & 30 & 10 & 65 & 90 & 45 & 53.33 & 1.00 \\
\hline 2 & $2-109-3$ & Bromus tectorum & cheatgrass & 1 & 2 & 9 & 4 & 1 & 3 & 1 & & 1 & 1 & 1 & 4 & 2.33 & 0.92 \\
\hline 2 & $2-109-3$ & Crepis atribarba & slender hawksbeard & 9 & 12 & 1 & 18 & 10 & & & & 2 & 6 & 8 & 13 & 6.58 & 0.75 \\
\hline 2 & $2-109-3$ & Erigeron filifolius & threadleaf fleabane & & 12 & & & 1 & 14 & & & & & & 10 & 3.08 & 0.33 \\
\hline 2 & $2-109-3$ & Festuca microstachys & small sixweeks & & & & & & & & & & 1 & & 1 & 0.17 & 0.17 \\
\hline 2 & $2-109-3$ & Lactuca serriola & prickly lettuce & & & & & & 1 & & & & & & & 0.08 & 0.08 \\
\hline 2 & $2-109-3$ & Litter & Litter & 25 & 8 & 10 & 6 & 16 & 15 & 20 & 16 & 10 & 16 & 8 & 14 & 13.67 & 1.00 \\
\hline 2 & $2-109-3$ & Lupinus sericeus & silky lupine & 2 & 16 & 1 & 13 & 1 & & 13 & 8 & 50 & & & & 8.67 & 0.67 \\
\hline 2 & $2-109-3$ & Phlox longifolia & longleaf phlox & & & & & 7 & 3 & & & 2 & & & 4 & 1.33 & 0.33 \\
\hline 2 & $2-109-3$ & Poa bulbosa & bulbous bluegrass & 2 & 2 & 12 & 7 & 4 & 1 & & 6 & & & & 1 & 2.92 & 0.67 \\
\hline 2 & $2-109-3$ & Poa sandbergii & Sandberg's bluegrass & 1 & 3 & 1 & 3 & 2 & 3 & 4 & 4 & & & & 5 & 2.17 & 0.75 \\
\hline 2 & $2-109-3$ & Sisymbrium altissimum & $\begin{array}{l}\text { Jim Hill's } \\
\text { tumblemustard }\end{array}$ & 5 & & 2 & & & & & & & & & & 0.58 & 0.17 \\
\hline 2 & $2-109-3$ & Stipa comata & needle-and-thread grass & & & & & & 4 & & & & & & & 0.33 & 0.08 \\
\hline 2 & $2-138-1$ & Agropyron spicatum & bluebunch wheatgrass & 1 & 2 & 1 & 4 & & & & & & & & & 0.67 & 0.33 \\
\hline 2 & $2-138-1$ & Amsinckia tessellata & devil's lettuce & 8 & 2 & & 2 & & & & & & & & & 1.00 & 0.25 \\
\hline 2 & $2-138-1$ & Artemisia tridentata & big sagebrush & 2 & 1 & 1 & 5 & & & & & & & & & 0.75 & 0.33 \\
\hline 2 & $2-138-1$ & Bare Ground & Bare Ground & 18 & 8 & 16 & 30 & & & & & & & & & 6.00 & 0.33 \\
\hline 2 & $2-138-1$ & Bromus tectorum & cheatgrass & 9 & & 5 & 8 & & & & & & & & & 1.83 & 0.25 \\
\hline 2 & $2-138-1$ & Chenopodium leptophyllum & slimleaf goosefoot & 1 & 5 & 6 & 4 & & & & & & & & & 1.33 & 0.33 \\
\hline 2 & $2-138-1$ & Litter & Litter & 8 & 6 & 10 & 4 & & & & & & & & & 2.33 & 0.33 \\
\hline 2 & $2-138-1$ & Poa sandbergii & Sandberg's bluegrass & & & & 10 & & & & & & & & & 0.83 & 0.08 \\
\hline 2 & $2-138-1$ & Salsola kali & Russian thistle & 51 & 60 & 50 & 35 & & & & & & & & & 16.33 & 0.33 \\
\hline 2 & $2-138-1$ & Sisymbrium altissimum & $\begin{array}{l}\text { Jim Hill's } \\
\text { tumblemustard }\end{array}$ & 32 & 56 & 45 & 10 & & & & & & & & & 11.92 & 0.33 \\
\hline 2 & $2-139-1$ & Agropyron spicatum & bluebunch wheatgrass & & & & & 2 & 1 & 3 & 3 & & & & & 0.75 & 0.33 \\
\hline
\end{tabular}


Table C.1. (contd)

\begin{tabular}{|c|c|c|c|c|c|c|c|c|c|c|c|c|c|c|c|c|c|}
\hline $\begin{array}{c}\text { Reveg } \\
\text { Unit }\end{array}$ & Site Name & Genus/Species & Common Name & $1 \mathrm{~m}$ & $5 \mathrm{~m}$ & $\begin{array}{l}10 \\
\mathrm{~m}\end{array}$ & $\begin{array}{l}15 \\
\mathrm{~m}\end{array}$ & $\begin{array}{l}18 \\
\mathrm{~m}\end{array}$ & $\begin{array}{c}22 \\
\mathrm{~m}\end{array}$ & $\begin{array}{c}26 \\
\mathrm{~m}\end{array}$ & $\begin{array}{l}30 \\
\mathrm{~m}\end{array}$ & $\begin{array}{l}35 \\
\mathrm{~m}\end{array}$ & $\begin{array}{l}40 \\
\mathrm{~m}\end{array}$ & $\begin{array}{c}44 \\
\mathrm{~m}\end{array}$ & $\begin{array}{l}48 \\
\mathrm{~m}\end{array}$ & $\begin{array}{l}\text { Mean } \\
\text { Cover }\end{array}$ & Frequency \\
\hline 2 & $2-139-1$ & Amsinckia tessellata & devil's lettuce & 2 & 7 & & & 10 & & 5 & 14 & & & & & 3.17 & 0.42 \\
\hline 2 & $2-139-1$ & Artemisia tridentata & big sagebrush & 1 & & 1 & 1 & & 4 & & & & & & & 0.58 & 0.33 \\
\hline 2 & $2-139-1$ & Bare Ground & Bare Ground & 30 & 35 & 36 & 40 & 30 & 40 & & 38 & & & & & 20.75 & 0.58 \\
\hline 2 & $2-139-1$ & Bromus tectorum & cheatgrass & 16 & & 5 & 10 & 14 & 3 & 1 & 3 & & & & & 4.33 & 0.58 \\
\hline 2 & $2-139-1$ & Chenopodium leptophyllum & slimleaf goosefoot & 3 & 1 & & 2 & 1 & 3 & 25 & 7 & & & & & 3.50 & 0.58 \\
\hline 2 & $2-139-1$ & Lactuca serriola & prickly lettuce & & & 2 & 3 & & & & 2 & & & & & 0.58 & 0.25 \\
\hline 2 & $2-139-1$ & Litter & Litter & 24 & 7 & 5 & 6 & 3 & 5 & & 6 & & & & & 4.67 & 0.58 \\
\hline 2 & $2-139-1$ & Salsola kali & Russian thistle & 30 & 38 & 50 & 35 & 38 & 46 & 17 & 30 & & & & & 23.67 & 0.67 \\
\hline 2 & $2-139-1$ & Sisymbrium altissimum & $\begin{array}{l}\text { Jim Hill's } \\
\text { tumblemustard }\end{array}$ & & 10 & & 4 & & 13 & 23 & 11 & & & & & 5.08 & 0.42 \\
\hline 2 & $2-139-1$ & Sitanion hystrix & bottlebrush grass & & 1 & 1 & & 2 & 1 & 6 & & & & & & 0.92 & 0.42 \\
\hline 2 & $2-50-1$ & Agropyron spicatum & bluebunch wheatgrass & & 13 & 6 & & 1 & 4 & 4 & 3 & 13 & 8 & 12 & 9 & 6.08 & 0.83 \\
\hline 2 & $2-50-1$ & Amsinckia lycopsoides & fiddleneck & 18 & 1 & & & & & 23 & & & & & & 3.50 & 0.25 \\
\hline 2 & $2-50-1$ & Bare Ground & Bare Ground & 1 & 18 & 31 & 50 & 77 & 44 & 50 & 55 & 84 & 63 & 80 & 90 & 53.58 & 1.00 \\
\hline 2 & $2-50-1$ & Bromus tectorum & cheatgrass & 6 & & 2 & & & & & & & & & & 0.67 & 0.17 \\
\hline 2 & $2-50-1$ & Calochortus macrocarpus & sagebrush mariposa lily & & 1 & & & & & & & & & & & 0.08 & 0.08 \\
\hline 2 & $2-50-1$ & Chrysothamnus nauseosus & gray rabbitbrush & & & & 8 & & 1 & & & & 3 & & & 1.00 & 0.25 \\
\hline 2 & $2-50-1$ & Crepis atribarba & slender hawksbeard & & 7 & 12 & 10 & 17 & 22 & 26 & 2 & 21 & & 1 & 8 & 10.50 & 0.83 \\
\hline 2 & $2-50-1$ & Descurainia pinnata & western tansymustard & & & 18 & & & & & & & & & & 1.50 & 0.08 \\
\hline 2 & $2-50-1$ & Erigeron piperianus & Piper's daisy & 2 & & & 1 & & & & & & & & & 0.25 & 0.17 \\
\hline 2 & $2-50-1$ & Helianthus cusickii & Cusick's sunflower & & & & 15 & 3 & & & & & 17 & & & 2.92 & 0.25 \\
\hline 2 & $2-50-1$ & Lactuca serriola & prickly lettuce & 2 & & & & & & & & & 1 & & & 0.25 & 0.17 \\
\hline 2 & $2-50-1$ & Litter & Litter & 87 & 48 & 10 & 3 & 5 & 7 & 4 & 3 & 2 & 14 & 5 & 1 & 15.75 & 1.00 \\
\hline 2 & $2-50-1$ & Lupinus sericeus & silky lupine & & & & & & & & & & 1 & & & 0.08 & 0.08 \\
\hline 2 & $2-50-1$ & Oryzopsis hymenoides & indian ricegrass & & & & & 3 & & 2 & & & & & & 0.42 & 0.17 \\
\hline 2 & $2-50-1$ & Phlox longifolia & longleaf phlox & & 6 & 2 & & & & & & & & & & 0.67 & 0.17 \\
\hline 2 & $2-50-1$ & Poa sandbergii & Sandberg's bluegrass & 4 & 6 & 18 & 23 & 10 & 29 & 7 & 40 & 15 & 13 & 10 & 4 & 14.92 & 1.00 \\
\hline 2 & $2-50-1$ & Salsola kali & Russian thistle & 15 & 1 & 1 & & & & 1 & & & 1 & 2 & 2 & 1.92 & 0.58 \\
\hline 2 & $2-50-1$ & Sisymbrium altissimum & $\begin{array}{l}\text { Jim Hill's } \\
\text { tumblemustard }\end{array}$ & 3 & & & & & & & & & & & & 0.25 & 0.08 \\
\hline 2 & $2-50-1$ & Stipa comata & needle-and-thread grass & & & & & & 4 & & & & 6 & & & 0.83 & 0.17 \\
\hline 2 & 2-DS146-X & Achillea millefolium & yarrow & & & & & & & & & & & & 1 & 0.08 & 0.08 \\
\hline
\end{tabular}


Table C.1. (contd)

\begin{tabular}{|c|c|c|c|c|c|c|c|c|c|c|c|c|c|c|c|c|c|}
\hline $\begin{array}{c}\text { Reveg } \\
\text { Unit }\end{array}$ & Site Name & Genus/Species & Common Name & $1 \mathrm{~m}$ & $5 \mathrm{~m}$ & $\begin{array}{l}10 \\
\mathrm{~m}\end{array}$ & $\begin{array}{l}15 \\
\mathrm{~m}\end{array}$ & $\begin{array}{l}18 \\
\mathrm{~m}\end{array}$ & $\begin{array}{l}22 \\
\mathrm{~m}\end{array}$ & $\begin{array}{l}26 \\
\mathrm{~m}\end{array}$ & $\begin{array}{l}30 \\
\mathrm{~m}\end{array}$ & $\begin{array}{l}35 \\
\mathrm{~m}\end{array}$ & $\begin{array}{l}40 \\
\mathrm{~m}\end{array}$ & $\begin{array}{l}44 \\
\mathrm{~m}\end{array}$ & $\begin{array}{l}48 \\
\mathrm{~m}\end{array}$ & $\begin{array}{l}\text { Mean } \\
\text { Cover }\end{array}$ & Frequency \\
\hline 2 & 2-DS146-X & Agropyron spicatum & bluebunch wheatgrass & 2 & & 2 & 5 & 4 & 2 & 7 & 2 & 3 & 2 & 1 & 1 & 2.58 & 0.92 \\
\hline 2 & 2-DS146-X & Amsinckia lycopsoides & fiddleneck & 1 & & 6 & 40 & & & & & 1 & 27 & & 10 & 7.08 & 0.50 \\
\hline 2 & 2-DS146-X & Artemisia tridentata & big sagebrush & & & & 1 & & & 4 & & 4 & 6 & 6 & 4 & 2.08 & 0.50 \\
\hline 2 & 2-DS146-X & Astragalus caricinus & buckwheat milkvetch & & 2 & & & & & & & & & & & 0.17 & 0.08 \\
\hline 2 & 2-DS146-X & Astragalus succumbens & crouching milkvetch & & 3 & 5 & & & & & & & & & & 0.67 & 0.17 \\
\hline 2 & 2-DS146-X & Bare Ground & Bare Ground & 90 & 75 & 60 & 30 & 35 & 62 & 70 & 50 & 45 & 40 & 75 & 65 & 58.08 & 1.00 \\
\hline 2 & 2-DS146-X & Bromus tectorum & cheatgrass & & & & & & 1 & & & & & & 2 & 0.25 & 0.17 \\
\hline 2 & 2-DS146-X & Chaenactis douglasii & hoary falseyarrow & & 4 & 4 & & & & & & & & & & 0.67 & 0.17 \\
\hline 2 & 2-DS146-X & Chenopodium leptophyllum & slimleaf goosefoot & & 1 & & 1 & & & & & & & & & 0.17 & 0.17 \\
\hline 2 & 2-DS146-X & Crepis atribarba & slender hawksbeard & 8 & 7 & & & & & & & & & & & 1.25 & 0.17 \\
\hline 2 & 2-DS146-X & Litter & Litter & 8 & & & & & 8 & 2 & 2 & 8 & 5 & 4 & 4 & 3.42 & 0.67 \\
\hline 2 & 2-DS146-X & Lupinus sericeus & silky lupine & & & 1 & & & 2 & & & & & & & 0.25 & 0.17 \\
\hline 2 & 2-DS146-X & Machaeranthera canescens & hoary aster & & 18 & 4 & & & & & & 2 & & & & 2.00 & 0.25 \\
\hline 2 & 2-DS146-X & Poa sandbergii & Sandberg's bluegrass & 3 & & 1 & 6 & 2 & & 4 & 4 & 4 & 1 & 2 & 3 & 2.50 & 0.83 \\
\hline 2 & 2-DS146-X & Salsola kali & Russian thistle & 18 & 10 & 22 & 50 & 65 & 35 & 20 & 75 & 60 & 20 & 28 & 28 & 35.92 & 1.00 \\
\hline 2 & 2-DS146-X & Sisymbrium altissimum & $\begin{array}{l}\text { Jim Hill's } \\
\text { tumblemustard }\end{array}$ & 2 & & 10 & 10 & 15 & & 6 & 6 & 16 & 26 & 15 & 9 & 9.58 & 0.83 \\
\hline 2 & 2-DS146-X & Sitanion hystrix & bottlebrush grass & & & & 1 & & & & & & & & & 0.08 & 0.08 \\
\hline 2 & 2-DS60-Xa & Achillea millefolium & yarrow & & 1 & & & & & & & & & & & 0.08 & 0.08 \\
\hline 2 & 2-DS60-Xa & Agropyron spicatum & bluebunch wheatgrass & & & 2 & 4 & & 2 & & & & & & & 0.67 & 0.25 \\
\hline 2 & 2-DS60-Xa & Ambrosia acanthicarpa & bur ragweed & & & & 1 & & & & & & & & & 0.08 & 0.08 \\
\hline 2 & 2-DS60-Xa & Amsinckia tessellata & devil's lettuce & & 6 & & & 1 & & & & & & & & 0.58 & 0.17 \\
\hline 2 & 2-DS60-Xa & Artemisia tridentata & big sagebrush & 2 & 1 & 1 & & & & & & & & & & 0.33 & 0.25 \\
\hline 2 & 2-DS60-Xa & Bare Ground & Bare Ground & 8 & 58 & 84 & 85 & 23 & 75 & & & & & & & 27.75 & 0.50 \\
\hline 2 & 2-DS60-Xa & Chenopodium leptophyllum & slimleaf goosefoot & & & & 2 & 2 & & & & & & & & 0.33 & 0.17 \\
\hline 2 & 2-DS60-Xa & Crepis atribarba & slender hawksbeard & 1 & & 8 & 6 & & 11 & & & & & & & 2.17 & 0.33 \\
\hline 2 & 2-DS60-Xa & Litter & Litter & 74 & 8 & 5 & 3 & 25 & 4 & & & & & & & 9.92 & 0.50 \\
\hline 2 & 2-DS60-Xa & Machaeranthera canescens & hoary aster & & 2 & 1 & 1 & & & & & & & & & 0.33 & 0.25 \\
\hline 2 & 2-DS60-Xa & Poa ampla Merr. & $\begin{array}{l}\text { 'Sherman' big } \\
\text { bluegrass }\end{array}$ & 1 & 1 & & & & 1 & & & & & & & 0.25 & 0.25 \\
\hline 2 & 2-DS60-Xa & Poa sandbergii & Sandberg's bluegrass & 2 & 3 & & 4 & 2 & 1 & & & & & & & 1.00 & 0.42 \\
\hline 2 & 2-DS60-Xa & Salsola kali & Russian thistle & & & & 1 & & & & & & & & & 0.08 & 0.08 \\
\hline
\end{tabular}


Table C.1. (contd)

\begin{tabular}{|c|c|c|c|c|c|c|c|c|c|c|c|c|c|c|c|c|c|}
\hline $\begin{array}{c}\text { Reveg } \\
\text { Unit }\end{array}$ & Site Name & Genus/Species & Common Name & $1 \mathrm{~m}$ & $5 \mathrm{~m}$ & $\begin{array}{l}10 \\
\mathrm{~m}\end{array}$ & $\begin{array}{l}15 \\
\mathrm{~m}\end{array}$ & $\begin{array}{l}18 \\
\mathrm{~m}\end{array}$ & $\begin{array}{c}22 \\
\mathrm{~m}\end{array}$ & $\begin{array}{c}26 \\
\mathrm{~m}\end{array}$ & $\begin{array}{l}30 \\
\mathrm{~m}\end{array}$ & $\begin{array}{l}35 \\
\mathrm{~m}\end{array}$ & $\begin{array}{l}40 \\
\mathrm{~m}\end{array}$ & $\begin{array}{c}44 \\
\mathrm{~m}\end{array}$ & $\begin{array}{c}48 \\
\mathrm{~m}\end{array}$ & $\begin{array}{l}\text { Mean } \\
\text { Cover }\end{array}$ & Frequency \\
\hline 2 & 2-DS60-Xa & Sisymbrium altissimum & $\begin{array}{l}\text { Jim Hill's } \\
\text { tumblemustard }\end{array}$ & 30 & 26 & 5 & 2 & 33 & 2 & & & & & & & 8.17 & 0.50 \\
\hline 2 & 2-DS60-Xa & Sitanion hystrix & bottlebrush grass & 10 & 18 & 3 & 1 & 11 & 8 & & & & & & & 4.25 & 0.50 \\
\hline 2 & 2-DS60-Xb & Agropyron spicatum & bluebunch wheatgrass & 10 & 4 & 2 & 2 & 1 & 4 & & & & & & & 1.92 & 0.50 \\
\hline 2 & 2-DS60-Xb & Ambrosia acanthicarpa & bur ragweed & 1 & 1 & & & & 1 & & & & & & & 0.25 & 0.25 \\
\hline 2 & 2-DS60-Xb & Artemisia tridentata & big sagebrush & 6 & 1 & 3 & 1 & & 3 & & & & & & & 1.17 & 0.42 \\
\hline 2 & 2-DS60-Xb & Astragalus succumbens & crouching milkvetch & & 3 & & & & & & & & & & & 0.25 & 0.08 \\
\hline 2 & 2-DS60-Xb & Balsamorhiza careyana & Carey's balsamroot & 1 & & & & & & & & & & & & 0.08 & 0.08 \\
\hline 2 & 2-DS60-Xb & Bare Ground & Bare Ground & 85 & 80 & 36 & 88 & 66 & 38 & & & & & & & 32.75 & 0.50 \\
\hline 2 & 2-DS60-Xb & Chenopodium leptophyllum & slimleaf goosefoot & 1 & 12 & & 1 & & 28 & & & & & & & 3.50 & 0.33 \\
\hline 2 & 2-DS60-Xb & Crepis atribarba & slender hawksbeard & & & & & & 5 & & & & & & & 0.42 & 0.08 \\
\hline 2 & 2-DS60-Xb & Festuca octoflora & slender sixweeks & & 1 & & & & & & & & & & & 0.08 & 0.08 \\
\hline 2 & 2-DS60-Xb & Litter & Litter & 2 & 8 & 50 & 1 & 8 & 40 & & & & & & & 9.08 & 0.50 \\
\hline 2 & 2-DS60-Xb & Machaeranthera canescens & hoary aster & 13 & 9 & 8 & 1 & 7 & & & & & & & & 3.17 & 0.42 \\
\hline 2 & 2-DS60-Xb & Poa ampla Merr. & $\begin{array}{l}\text { 'Sherman' big } \\
\text { bluegrass }\end{array}$ & & 6 & 2 & 1 & 5 & & & & & & & & 1.17 & 0.33 \\
\hline 2 & 2-DS60-Xb & Poa sandbergii & Sandberg's bluegrass & 6 & 48 & 4 & 2 & 2 & 1 & & & & & & & 5.25 & 0.50 \\
\hline 2 & 2-DS60-Xb & Sisymbrium altissimum & $\begin{array}{l}\text { Jim Hill's } \\
\text { tumblemustard }\end{array}$ & & 4 & & 3 & 18 & 5 & & & & & & & 2.50 & 0.33 \\
\hline 2 & 2-DS60-Xb & Sitanion hystrix & bottlebrush grass & & 1 & 6 & 1 & 1 & & & & & & & & 0.75 & 0.33 \\
\hline 2 & 2-HQ-1 & Achillea millefolium & yarrow & & & 1 & 2 & 3 & & & & & & & 1 & 0.58 & 0.33 \\
\hline 2 & 2-HQ-1 & Agropyron spicatum & bluebunch wheatgrass & 1 & 1 & & 1 & 1 & & & 4 & 1 & 1 & 1 & & 0.92 & 0.67 \\
\hline 2 & 2-HQ-1 & Artemisia tridentata & big sagebrush & & & 4 & & & & & & & 1 & & & 0.42 & 0.17 \\
\hline 2 & 2-HQ-1 & Bare Ground & Bare Ground & 10 & 10 & 6 & 4 & 18 & 45 & 60 & & 40 & 7 & 90 & 20 & 25.83 & 0.92 \\
\hline 2 & 2-HQ-1 & Erigeron piperianus & Piper's daisy & & & & 1 & & & & & & & & & 0.08 & 0.08 \\
\hline 2 & 2-HQ-1 & Litter & Litter & 85 & 90 & 90 & 95 & 65 & 45 & 45 & 75 & 54 & 70 & 10 & 72 & 66.33 & 1.00 \\
\hline 2 & 2-HQ-1 & Melilotus sp. & & & & & 1 & 1 & & & & & & & & 0.17 & 0.17 \\
\hline 2 & 2-HQ-1 & Phlox longifolia & longleaf phlox & & & & & & & 1 & & & & & & 0.08 & 0.08 \\
\hline 2 & 2-HQ-1 & Poa sandbergii & Sandberg's bluegrass & 3 & 1 & 1 & 1 & 1 & 2 & 2 & 1 & 1 & & 1 & 1 & 1.25 & 0.92 \\
\hline 2 & 2-HQ-1 & Salsola kali & Russian thistle & 21 & 28 & 18 & 20 & 20 & 15 & 12 & 20 & 2 & 2 & 18 & 2 & 14.83 & 1.00 \\
\hline 2 & 2-HQ-1 & Sitanion hystrix & bottlebrush grass & 2 & 5 & 8 & 69 & 10 & 12 & 10 & 7 & 10 & 12 & 4 & 4 & 12.75 & 1.00 \\
\hline
\end{tabular}


Table C.1. (contd)

\begin{tabular}{|c|c|c|c|c|c|c|c|c|c|c|c|c|c|c|c|c|c|}
\hline $\begin{array}{c}\text { Reveg } \\
\text { Unit }\end{array}$ & Site Name & Genus/Species & Common Name & $1 \mathrm{~m}$ & $5 \mathrm{~m}$ & $\begin{array}{l}10 \\
\mathrm{~m}\end{array}$ & $\begin{array}{l}15 \\
\mathrm{~m}\end{array}$ & $\begin{array}{l}18 \\
\mathrm{~m}\end{array}$ & $\begin{array}{l}22 \\
\mathrm{~m}\end{array}$ & $\begin{array}{c}26 \\
\mathrm{~m}\end{array}$ & $\begin{array}{l}30 \\
\mathrm{~m}\end{array}$ & $\begin{array}{l}35 \\
\mathrm{~m}\end{array}$ & $\begin{array}{l}40 \\
\mathrm{~m}\end{array}$ & $\begin{array}{l}44 \\
\mathrm{~m}\end{array}$ & $\begin{array}{r}48 \\
\mathrm{~m}\end{array}$ & $\begin{array}{l}\text { Mean } \\
\text { Cover }\end{array}$ & Frequency \\
\hline 2 & 2-HQ-2 & Achillea millefolium & yarrow & & 1 & & & & & & & 1 & & & 1 & 0.25 & 0.25 \\
\hline 2 & 2-HQ-2 & Agropyron spicatum & bluebunch wheatgrass & 1 & 2 & 2 & 2 & 2 & 2 & 2 & 2 & 2 & 1 & 2 & 2 & 1.83 & 1.00 \\
\hline 2 & 2-HQ-2 & Bare Ground & Bare Ground & 2 & 20 & 20 & 16 & 8 & 6 & 55 & 75 & 24 & 65 & 16 & 35 & 28.50 & 1.00 \\
\hline 2 & 2-HQ-2 & Lactuca serriola & prickly lettuce & & & & & & & & & 2 & & 1 & & 0.25 & 0.17 \\
\hline 2 & 2-HQ-2 & Litter & Litter & 95 & 70 & 34 & 65 & 85 & 90 & 6 & 16 & 75 & 20 & 60 & 55 & 55.92 & 1.00 \\
\hline 2 & 2-HQ-2 & Poa sandbergii & Sandberg's bluegrass & 1 & 3 & 6 & 3 & 2 & 2 & 3 & 2 & 2 & & & 3 & 2.25 & 0.83 \\
\hline 2 & 2-HQ-2 & Salsola kali & Russian thistle & 1 & 3 & 7 & 14 & 18 & 8 & 35 & 2 & 6 & 15 & 32 & 2 & 11.92 & 1.00 \\
\hline 2 & 2-HQ-2 & Sisymbrium altissimum & $\begin{array}{l}\text { Jim Hill's } \\
\text { tumblemustard }\end{array}$ & 1 & & & & & & & & & & 6 & 1 & 0.67 & 0.25 \\
\hline 2 & 2-HQ-2 & Sitanion hystrix & bottlebrush grass & 8 & 3 & 3 & 1 & 2 & 6 & 2 & 2 & 4 & 1 & 3 & 4 & 3.25 & 1.00 \\
\hline 2 & 2-HQ-2 & Vulpia myuros (L.) C.C. Gmel & rat-tail fescue & & & & & & & & 2 & & & 2 & & 0.33 & 0.17 \\
\hline 2 & 2-HQ-3 & Achillea millefolium & yarrow & & & 1 & & 1 & & & 2 & & & & & 0.33 & 0.25 \\
\hline 2 & 2-HQ-3 & Agropyron spicatum & bluebunch wheatgrass & 1 & 1 & 1 & 1 & & 2 & 2 & 2 & 2 & 2 & 2 & 2 & 1.50 & 0.92 \\
\hline 2 & 2-HQ-3 & Artemisia tridentata & big sagebrush & & & & & & & 1 & 1 & 1 & & & & 0.25 & 0.25 \\
\hline 2 & 2-HQ-3 & Balsamorhiza careyana & Carey's balsamroot & & & 1 & & 1 & & & & 1 & & & & 0.25 & 0.25 \\
\hline 2 & 2-HQ-3 & Bare Ground & Bare Ground & 5 & 3 & 75 & 60 & 30 & 50 & 50 & 4 & 6 & 2 & 10 & 54 & 29.08 & 1.00 \\
\hline 2 & 2-HQ-3 & Bromus tectorum & cheatgrass & & & & & & & 1 & 1 & & 1 & & & 0.25 & 0.25 \\
\hline 2 & 2-HQ-3 & Chenopodium leptophyllum & slimleaf goosefoot & & & & & & & & & 1 & & & & 0.08 & 0.08 \\
\hline 2 & 2-HQ-3 & Festuca octoflora & slender sixweeks & & & 1 & & 2 & & & & & & & & 0.25 & 0.17 \\
\hline 2 & 2-HQ-3 & Litter & Litter & 95 & 95 & 14 & 30 & 50 & 45 & 24 & 88 & 85 & 97 & 90 & 42 & 62.92 & 1.00 \\
\hline 2 & 2-HQ-3 & Machaeranthera canescens & hoary aster & & & & & 1 & & & 3 & & & & & 0.33 & 0.17 \\
\hline 2 & 2-HQ-3 & Melilotus alba & white sweetclover & & & 1 & & & & & & & & & & 0.08 & 0.08 \\
\hline 2 & 2-HQ-3 & Poa sandbergii & Sandberg's bluegrass & & & 1 & 2 & & 2 & 1 & & & & & & 0.50 & 0.33 \\
\hline 2 & 2-HQ-3 & Salsola kali & Russian thistle & & 24 & 14 & & 26 & 8 & 22 & 9 & 10 & 6 & 4 & 6 & 10.75 & 0.83 \\
\hline 2 & 2-HQ-3 & Sisymbrium altissimum & $\begin{array}{l}\text { Jim Hill's } \\
\text { tumblemustard }\end{array}$ & 1 & 2 & 15 & & 2 & 5 & & 8 & 16 & 1 & & & 4.17 & 0.67 \\
\hline 2 & 2-HQ-3 & Sitanion hystrix & bottlebrush grass & 18 & 15 & 8 & 4 & 2 & 7 & 5 & 10 & 6 & 6 & 6 & 7 & 7.83 & 1.00 \\
\hline 2 & 2-HQ-3 & Vulpia myuros (L.) C.C. Gmel & rat-tail fescue & & 1 & & & & & & 3 & 2 & & & & 0.50 & 0.25 \\
\hline 2 & 2-HW-1 & Agropyron spicatum & bluebunch wheatgrass & 2 & 1 & & 1 & 2 & 5 & 5 & 2 & 2 & 5 & 4 & 3 & 2.67 & 0.92 \\
\hline 2 & 2-HW-1 & Amsinckia lycopsoides & fiddleneck & 12 & 8 & 10 & 20 & 3 & 6 & 22 & 11 & 11 & 28 & 35 & & 13.83 & 0.92 \\
\hline 2 & 2-HW-1 & Artemisia tridentata & big sagebrush & & & & & & 4 & & & & & & 1 & 0.42 & 0.17 \\
\hline 2 & 2-HW-1 & Balsamorhiza careyana & Carey's balsamroot & & & & & & & & & & 1 & & & 0.08 & 0.08 \\
\hline
\end{tabular}


Table C.1. (contd)

\begin{tabular}{|c|c|c|c|c|c|c|c|c|c|c|c|c|c|c|c|c|c|}
\hline $\begin{array}{l}\text { Reveg } \\
\text { Unit }\end{array}$ & Site Name & Genus/Species & Common Name & $1 \mathrm{~m}$ & $5 \mathrm{~m}$ & $\begin{array}{l}10 \\
\mathrm{~m}\end{array}$ & $\begin{array}{l}15 \\
\mathrm{~m}\end{array}$ & $\begin{array}{l}18 \\
\mathrm{~m}\end{array}$ & $\begin{array}{l}22 \\
\mathrm{~m}\end{array}$ & $\begin{array}{l}26 \\
\mathrm{~m}\end{array}$ & $\begin{array}{l}30 \\
\mathrm{~m}\end{array}$ & $\begin{array}{l}35 \\
\mathrm{~m}\end{array}$ & $\begin{array}{l}40 \\
\mathrm{~m}\end{array}$ & $\begin{array}{l}44 \\
\mathrm{~m}\end{array}$ & $\begin{array}{l}48 \\
\mathrm{~m}\end{array}$ & $\begin{array}{l}\text { Mean } \\
\text { Cover }\end{array}$ & Frequency \\
\hline 2 & $2-\mathrm{HW}-1$ & Bare Ground & Bare Ground & 4 & 3 & 2 & 18 & 80 & 6 & 26 & 6 & 1 & 8 & 6 & 4 & 13.67 & 1.00 \\
\hline 2 & 2-HW-1 & Bromus tectorum & cheatgrass & 2 & & & & & & & & & & & 3 & 0.42 & 0.17 \\
\hline 2 & 2-HW-1 & Chenopodium leptophyllum & slimleaf goosefoot & 1 & 1 & 1 & 1 & 1 & & & 1 & 1 & & 1 & 1 & 0.75 & 0.75 \\
\hline 2 & 2-HW-1 & Crepis atribarba & slender hawksbeard & 6 & & & & & & & & & & & 33 & 3.25 & 0.17 \\
\hline 2 & 2-HW-1 & Descurainia pinnata & western tansymustard & & & & 6 & & & & 4 & & & & & 0.83 & 0.17 \\
\hline 2 & 2-HW-1 & Erodium cicutarium & storksbill & & & & & 1 & & & & & & & & 0.08 & 0.08 \\
\hline 2 & 2-HW-1 & Lactuca serriola & prickly lettuce & & 1 & 2 & & & & 3 & & 1 & & & & 0.58 & 0.33 \\
\hline 2 & 2-HW-1 & Litter & Litter & 92 & 84 & 87 & 30 & 10 & 70 & 7 & 30 & 90 & 8 & 38 & 44 & 49.17 & 1.00 \\
\hline 2 & 2-HW-1 & Poa sandbergii & Sandberg's bluegrass & 2 & 3 & & 2 & 3 & 4 & 2 & 1 & 1 & 4 & 4 & 2 & 2.33 & 0.92 \\
\hline 2 & 2-HW-1 & Salsola kali & Russian thistle & 1 & & 14 & & 1 & & & & & 8 & & & 2.00 & 0.33 \\
\hline 2 & 2-HW-1 & Sisymbrium altissimum & $\begin{array}{l}\text { Jim Hill's } \\
\text { tumblemustard }\end{array}$ & 20 & 84 & 20 & 65 & 20 & 60 & 95 & 92 & 74 & 96 & 22 & 15 & 55.25 & 1.00 \\
\hline 2 & 2-HW-1 & Sitanion hystrix & bottlebrush grass & 20 & 5 & 10 & 12 & 4 & 24 & 8 & 5 & 13 & 22 & 25 & 14 & 13.50 & 1.00 \\
\hline 2 & 2-HW-2 & Agropyron spicatum & bluebunch wheatgrass & 1 & 2 & 2 & & 2 & 2 & 1 & 1 & 6 & 4 & 1 & & 1.83 & 0.83 \\
\hline 2 & 2-HW-2 & Amsinckia lycopsoides & fiddleneck & 10 & & & & & 4 & 4 & 10 & 1 & & 6 & 30 & 5.42 & 0.58 \\
\hline 2 & 2-HW-2 & Artemisia tridentata & big sagebrush & & & & & & & & 2 & & & & & 0.17 & 0.08 \\
\hline 2 & 2-HW-2 & Bare Ground & Bare Ground & 8 & 20 & 80 & & 85 & 8 & & 20 & 8 & 5 & 12 & 8 & 21.17 & 0.83 \\
\hline 2 & 2-HW-2 & Bromus tectorum & cheatgrass & & & & & & 2 & 22 & 4 & & & & & 2.33 & 0.25 \\
\hline 2 & 2-HW-2 & Chenopodium leptophyllum & slimleaf goosefoot & & & 1 & & & & & & 1 & & & 1 & 0.25 & 0.25 \\
\hline 2 & 2-HW-2 & Descurainia pinnata & western tansymustard & & & & & & & & & 1 & & & & 0.08 & 0.08 \\
\hline 2 & 2-HW-2 & Lactuca serriola & prickly lettuce & 2 & 2 & 6 & 6 & & 6 & 2 & & 3 & 1 & & 2 & 2.50 & 0.75 \\
\hline 2 & 2-HW-2 & Litter & Litter & 75 & 60 & 20 & & 8 & 40 & 95 & 42 & 80 & 78 & 50 & 45 & 49.42 & 0.92 \\
\hline 2 & 2-HW-2 & Lupinus sericeus & silky lupine & & 1 & & & & & & & & & & & 0.08 & 0.08 \\
\hline 2 & 2-HW-2 & Machaeranthera canescens & hoary aster & 1 & & & & & & & 1 & 4 & & & 4 & 0.83 & 0.33 \\
\hline 2 & 2-HW-2 & Poa sandbergii & Sandberg's bluegrass & & & 2 & & 6 & & & 1 & 1 & & 1 & & 0.92 & 0.42 \\
\hline 2 & 2-HW-2 & Salsola kali & Russian thistle & & & & & 3 & & & & & & 8 & & 0.92 & 0.17 \\
\hline 2 & 2-HW-2 & Sisymbrium altissimum & $\begin{array}{l}\text { Jim Hill's } \\
\text { tumblemustard }\end{array}$ & 12 & 2 & 2 & 4 & 10 & 30 & 2 & 22 & & 6 & 48 & 6 & 12.00 & 0.92 \\
\hline 2 & 2-HW-2 & Sitanion hystrix & bottlebrush grass & 10 & 4 & 6 & & & 2 & 86 & 6 & 4 & 20 & 15 & 6 & 13.25 & 0.83 \\
\hline 3 & 3-RT-1 & Agastache occidentalis & western horsemint & & & & & & & 1 & & & & & & 0.08 & 0.08 \\
\hline 3 & 3-RT-1 & Agropyron spicatum & bluebunch wheatgrass & 2 & 3 & 2 & 6 & 2 & 3 & 4 & 13 & 6 & 32 & 6 & 5 & 7.00 & 1.00 \\
\hline 3 & 3-RT-1 & Bare Ground & Bare Ground & 97 & 90 & 92 & 90 & 80 & 95 & 78 & 80 & 83 & 83 & 86 & 84 & 86.50 & 1.00 \\
\hline
\end{tabular}


Table C.1. (contd)

\begin{tabular}{|c|c|c|c|c|c|c|c|c|c|c|c|c|c|c|c|c|c|}
\hline $\begin{array}{l}\text { Reveg } \\
\text { Unit }\end{array}$ & Site Name & Genus/Species & Common Name & $1 \mathrm{~m}$ & $5 \mathrm{~m}$ & $\begin{array}{l}10 \\
\mathrm{~m}\end{array}$ & $\begin{array}{l}15 \\
\mathrm{~m}\end{array}$ & $\begin{array}{l}18 \\
\mathrm{~m}\end{array}$ & $\begin{array}{l}22 \\
\mathrm{~m}\end{array}$ & $\begin{array}{l}26 \\
\mathrm{~m}\end{array}$ & $\begin{array}{l}30 \\
\mathrm{~m}\end{array}$ & $\begin{array}{l}35 \\
\mathrm{~m}\end{array}$ & $\begin{array}{l}40 \\
\mathrm{~m}\end{array}$ & $\begin{array}{l}44 \\
\mathrm{~m}\end{array}$ & $\begin{array}{l}48 \\
\mathrm{~m}\end{array}$ & $\begin{array}{l}\text { Mean } \\
\text { Cover }\end{array}$ & Frequency \\
\hline 3 & 3-RT-1 & Bromus tectorum & cheatgrass & & & 1 & 1 & 45 & 5 & 24 & 11 & 34 & & 7 & 20 & 12.33 & 0.75 \\
\hline 3 & 3-RT-1 & Chenopodium leptophyllum & slimleaf goosefoot & & & & & & 2 & & & & & & & 0.17 & 0.08 \\
\hline 3 & 3-RT-1 & Crepis atribarba & slender hawksbeard & & & & & 2 & & & & & & & & 0.17 & 0.08 \\
\hline 3 & 3-RT-1 & Festuca idahoensis & Idaho fescue & 1 & & & & & & & & & 13 & & & 1.17 & 0.17 \\
\hline 3 & 3-RT-1 & Litter & Litter & & 6 & & 2 & 1 & 1 & 2 & & 1 & & 1 & 4 & 1.50 & 0.67 \\
\hline 3 & 3-RT-1 & Lomatium grayi & Gray's desertparsley & & & & & & 1 & & & & & & & 0.08 & 0.08 \\
\hline 3 & 3-RT-1 & Phacelia hastata & whiteleaf scorpionweed & & & 1 & & & & & 2 & & & & 1 & 0.33 & 0.25 \\
\hline 3 & 3-RT-1 & Poa sandbergii & Sandberg's bluegrass & 6 & 2 & 4 & 2 & 4 & 2 & 3 & 12 & 1 & 4 & 4 & 2 & 3.83 & 1.00 \\
\hline 3 & 3-RT-1 & Salsola kali & Russian thistle & 1 & & & & & & & & & & & & 0.08 & 0.08 \\
\hline 3 & 3-RT-1 & Sisymbrium altissimum & $\begin{array}{l}\text { Jim Hill's } \\
\text { tumblemustard }\end{array}$ & & & & & 4 & 2 & & & & 1 & 2 & 2 & 0.92 & 0.42 \\
\hline 3 & 3-RT-2 & Agropyron spicatum & bluebunch wheatgrass & 3 & 4 & 2 & 4 & 6 & 2 & 6 & 4 & 2 & 1 & & 1 & 2.92 & 0.92 \\
\hline 3 & 3-RT-2 & Bare Ground & Bare Ground & 85 & 92 & 95 & 88 & 90 & 94 & 90 & 85 & 82 & 96 & 98 & 99 & 91.17 & 1.00 \\
\hline 3 & 3-RT-2 & Bromus tectorum & cheatgrass & 14 & 6 & 2 & 4 & 1 & 2 & 2 & 34 & 1 & & & & 5.50 & 0.75 \\
\hline 3 & 3-RT-2 & Chenopodium leptophyllum & slimleaf goosefoot & 1 & 1 & & 1 & 1 & & & & & & & & 0.33 & 0.33 \\
\hline 3 & 3-RT-2 & Festuca idahoensis & Idaho fescue & & 1 & & 1 & 1 & & 1 & 1 & & & 1 & & 0.50 & 0.50 \\
\hline 3 & 3-RT-2 & Litter & Litter & & & & 1 & & & 1 & & & & & & 0.17 & 0.17 \\
\hline 3 & 3-RT-2 & Lupinus sericeus & silky lupine & & & & & & & 1 & & & & & & 0.08 & 0.08 \\
\hline 3 & 3-RT-2 & Phacelia hastata & whiteleaf scorpionweed & & 1 & & & 1 & 1 & 1 & & & & & & 0.33 & 0.33 \\
\hline 3 & 3-RT-2 & Poa sandbergii & Sandberg's bluegrass & 4 & 4 & 6 & 6 & 6 & 5 & 4 & 5 & 3 & 1 & 1 & 1 & 3.83 & 1.00 \\
\hline 3 & 3-RT-2 & Salsola kali & Russian thistle & & & & & & & & 10 & 2 & 1 & 1 & & 1.17 & 0.33 \\
\hline 3 & 3-RT-2 & Sisymbrium altissimum & $\begin{array}{l}\text { Jim Hill's } \\
\text { tumblemustard }\end{array}$ & & 1 & & 1 & & & & & & & & & 0.17 & 0.17 \\
\hline 3 & 3-RT-3 & Agropyron spicatum & bluebunch wheatgrass & 5 & 2 & 6 & 5 & 8 & 10 & 7 & 8 & 4 & 7 & 10 & 12 & 7.00 & 1.00 \\
\hline 3 & $3-\mathrm{RT}-3$ & Bare Ground & Bare Ground & 90 & 98 & 92 & 98 & 96 & 95 & 97 & 96 & 98 & 95 & 92 & 93 & 95.00 & 1.00 \\
\hline 3 & 3-RT-3 & Bromus japonicus & Japanese brome & & & & & & & & 4 & & & & & 0.33 & 0.08 \\
\hline 3 & 3-RT-3 & Bromus tectorum & cheatgrass & 2 & 2 & 2 & & & 2 & 3 & 1 & 6 & & & & 1.50 & 0.58 \\
\hline 3 & 3-RT-3 & Crepis modocensis & low hawksbeard & & & & & 1 & & & & & & & & 0.08 & 0.08 \\
\hline 3 & $3-\mathrm{RT}-3$ & Litter & Litter & 2 & & 1 & 1 & & 1 & 1 & 2 & & 1 & & 1 & 0.83 & 0.67 \\
\hline 3 & $3-\mathrm{RT}-3$ & Phacelia hastata & whiteleaf scorpionweed & & & & & & & & & 1 & & & 1 & 0.17 & 0.17 \\
\hline 3 & 3-RT-3 & Poa sandbergii & Sandberg's bluegrass & 6 & 1 & 2 & 1 & 2 & 1 & 2 & 3 & 1 & 5 & 2 & 3 & 2.42 & 1.00 \\
\hline 3 & 3-RT-3 & Salsola kali & Russian thistle & & & & & & 1 & 1 & & 3 & & 2 & 1 & 0.67 & 0.42 \\
\hline
\end{tabular}


Table C.1. (contd)

\begin{tabular}{|c|c|c|c|c|c|c|c|c|c|c|c|c|c|c|c|c|c|}
\hline $\begin{array}{l}\text { Reveg } \\
\text { Unit }\end{array}$ & Site Name & Genus/Species & Common Name & $1 \mathrm{~m}$ & $5 \mathrm{~m}$ & $\begin{array}{l}10 \\
\mathrm{~m}\end{array}$ & $\begin{array}{l}15 \\
\mathrm{~m}\end{array}$ & $\begin{array}{l}18 \\
\mathrm{~m}\end{array}$ & $\begin{array}{l}22 \\
\mathrm{~m}\end{array}$ & $\begin{array}{l}26 \\
\mathrm{~m}\end{array}$ & $\begin{array}{l}30 \\
\mathrm{~m}\end{array}$ & $\begin{array}{l}35 \\
\mathrm{~m}\end{array}$ & $\begin{array}{l}40 \\
\mathrm{~m}\end{array}$ & $\begin{array}{l}44 \\
\mathrm{~m}\end{array}$ & $\begin{array}{l}48 \\
\mathrm{~m}\end{array}$ & $\begin{array}{l}\text { Mean } \\
\text { Cover }\end{array}$ & Frequency \\
\hline 3 & 3-RT-3 & Sisymbrium altissimum & $\begin{array}{l}\text { Jim Hill's } \\
\text { tumblemustard }\end{array}$ & 2 & & & & & & & & & & & 2 & 0.33 & 0.17 \\
\hline 4 & $4-623 \mathrm{~A}-1$ & Agropyron spicatum & bluebunch wheatgrass & & & 2 & & & & & & & & & & 0.17 & 0.08 \\
\hline 4 & $4-623 \mathrm{~A}-1$ & Bare Ground & Bare Ground & 100 & 98 & 99 & 99 & 100 & 98 & 99 & 98 & 100 & 100 & 100 & 99 & 99.17 & 1.00 \\
\hline 4 & $4-623 \mathrm{~A}-1$ & Bromus tectorum & cheatgrass & & & & & & 5 & & 1 & & & & & 0.50 & 0.17 \\
\hline 4 & $4-623 \mathrm{~A}-1$ & Litter & Litter & 1 & & & 4 & 3 & 2 & 1 & 4 & 3 & & & & 1.50 & 0.58 \\
\hline 4 & $4-623 \mathrm{~A}-1$ & Phacelia hastata & whiteleaf scorpionweed & & & 1 & & & & & & & & & & 0.08 & 0.08 \\
\hline 4 & $4-623 \mathrm{~A}-1$ & Poa sandbergii & Sandberg's bluegrass & 1 & & & 1 & 1 & & 1 & 1 & & 1 & 1 & 1 & 0.67 & 0.67 \\
\hline 4 & $4-623 \mathrm{~A}-1$ & Polygonum aviculare & doorweed & & & & & & & 1 & & & & & & 0.08 & 0.08 \\
\hline 4 & $4-623 \mathrm{~A}-1$ & Salsola kali & Russian thistle & & 10 & & 2 & & 2 & & & & & & 1 & 1.25 & 0.33 \\
\hline 4 & $4-623 \mathrm{~A}-1$ & Sisymbrium altissimum & $\begin{array}{l}\text { Jim Hill's } \\
\text { tumblemustard }\end{array}$ & & & & & & 1 & & & & & & & 0.08 & 0.08 \\
\hline 4 & 4-623A-road & Achillea millefolium & yarrow & & & & 1 & & & & & & & & & 0.08 & 0.08 \\
\hline 4 & 4-623A-road & Agropyron spicatum & bluebunch wheatgrass & 1 & 1 & & 1 & 3 & & & & & & & & 0.50 & 0.33 \\
\hline 4 & 4-623A-road & Balsamorhiza careyana & Carey's balsamroot & & & & & 9 & & & & & & & & 0.75 & 0.08 \\
\hline 4 & 4-623A-road & Balsamorhiza rosea & rosy balsamroot & & & & 1 & & & & & & & & & 0.08 & 0.08 \\
\hline 4 & 4-623A-road & Bare Ground & Bare Ground & 95 & 90 & 88 & 85 & 80 & 97 & & & & & & & 44.58 & 0.50 \\
\hline 4 & 4-623A-road & Bromus tectorum & cheatgrass & 5 & 3 & 21 & 23 & 10 & & & & & & & & 5.17 & 0.42 \\
\hline 4 & 4-623A-road & Litter & Litter & 1 & 4 & 2 & 2 & 4 & & & & & & & & 1.08 & 0.42 \\
\hline 4 & 4-623A-road & Lupinus sericeus & silky lupine & & 1 & & & 5 & 1 & & & & & & & 0.58 & 0.25 \\
\hline 4 & 4-623A-road & Phacelia hastata & whiteleaf scorpionweed & 4 & & & & & & & & & & & & 0.33 & 0.08 \\
\hline 4 & 4-623A-road & Poa sandbergii & Sandberg's bluegrass & 3 & 1 & 7 & 10 & 12 & 5 & & & & & & & 3.17 & 0.50 \\
\hline 4 & 4-623A-road & Salsola kali & Russian thistle & 1 & 12 & & 1 & 25 & 4 & & & & & & & 3.58 & 0.42 \\
\hline 4 & 4-623A-road & Sisymbrium altissimum & $\begin{array}{l}\text { Jim Hill's } \\
\text { tumblemustard }\end{array}$ & & & 7 & 5 & & 1 & & & & & & & 1.08 & 0.25 \\
\hline 4 & 4-623A-road & Sitanion hystrix & bottlebrush grass & 5 & & & 2 & & & & & & & & & 0.58 & 0.17 \\
\hline 4 & $4-\mathrm{C}-1$ & Agropyron spicatum & bluebunch wheatgrass & 4 & 1 & & & 1 & 1 & 1 & & 4 & 2 & 2 & 2 & 1.50 & 0.75 \\
\hline 4 & 4-C-1 & Bare Ground & Bare Ground & 92 & 90 & 99 & 98 & 97 & 97 & 98 & 93 & 92 & 97 & 85 & 90 & 94.00 & 1.00 \\
\hline 4 & $4-\mathrm{C}-1$ & Festuca idahoensis & Idaho fescue & 1 & 1 & & & 1 & 1 & 1 & & 4 & 2 & 2 & 4 & 1.42 & 0.75 \\
\hline 4 & $4-\mathrm{C}-1$ & Litter & Litter & & & & & 1 & 1 & 1 & 1 & 1 & & & & 0.42 & 0.42 \\
\hline 4 & $4-\mathrm{C}-1$ & Phacelia hastata & whiteleaf scorpionweed & 2 & 2 & & & 1 & & & & & 1 & 2 & 2 & 0.83 & 0.50 \\
\hline
\end{tabular}


Table C.1. (contd)

\begin{tabular}{|c|c|c|c|c|c|c|c|c|c|c|c|c|c|c|c|c|c|}
\hline $\begin{array}{l}\text { Reveg } \\
\text { Unit }\end{array}$ & Site Name & Genus/Species & Common Name & $1 \mathrm{~m}$ & $5 \mathrm{~m}$ & $\begin{array}{l}10 \\
\mathrm{~m}\end{array}$ & $\begin{array}{l}15 \\
\mathrm{~m}\end{array}$ & $\begin{array}{l}18 \\
\mathrm{~m}\end{array}$ & $\begin{array}{l}22 \\
\mathrm{~m}\end{array}$ & $\begin{array}{l}26 \\
\mathrm{~m}\end{array}$ & $\begin{array}{l}30 \\
\mathrm{~m}\end{array}$ & $\begin{array}{l}35 \\
\mathrm{~m}\end{array}$ & $\begin{array}{l}40 \\
\mathrm{~m}\end{array}$ & $\begin{array}{l}44 \\
\mathrm{~m}\end{array}$ & $\begin{array}{l}48 \\
\mathrm{~m}\end{array}$ & $\begin{array}{l}\text { Mean } \\
\text { Cover }\end{array}$ & Frequency \\
\hline 4 & $4-\mathrm{C}-1$ & Poa sandbergii & Sandberg's bluegrass & 4 & 4 & 2 & 4 & 2 & 2 & 4 & 6 & 2 & 1 & 10 & 4 & 3.75 & 1.00 \\
\hline 4 & $4-\mathrm{C}-1$ & Salsola kali & Russian thistle & 2 & 6 & & & & & & 1 & 1 & 1 & & & 0.92 & 0.42 \\
\hline 4 & $4-C-2$ & Agropyron spicatum & bluebunch wheatgrass & 6 & 4 & 10 & 3 & 1 & 2 & 1 & 4 & 6 & 7 & 1 & 2 & 3.92 & 1.00 \\
\hline 4 & $4-\mathrm{C}-2$ & Bare Ground & Bare Ground & 85 & 77 & 75 & 80 & 68 & 85 & 93 & 83 & 66 & 58 & 94 & 89 & 79.42 & 1.00 \\
\hline 4 & $4-\mathrm{C}-2$ & Bromus tectorum & cheatgrass & & 4 & 4 & 2 & 1 & 9 & 7 & 6 & & 4 & & & 3.08 & 0.67 \\
\hline 4 & $4-\mathrm{C}-2$ & Chenopodium leptophyllum & slimleaf goosefoot & 1 & & & & & & & & & & & & 0.08 & 0.08 \\
\hline 4 & $4-\mathrm{C}-2$ & Festuca idahoensis & Idaho fescue & & 1 & 1 & 1 & & & & 1 & & 1 & & 1 & 0.50 & 0.50 \\
\hline 4 & $4-\mathrm{C}-2$ & Litter & Litter & 0 & 2 & 3 & 4 & 1 & 1 & 1 & 4 & 5 & 1 & & 1 & 1.92 & 0.83 \\
\hline 4 & $4-\mathrm{C}-2$ & Phacelia hastata & whiteleaf scorpionweed & 8 & 6 & & 1 & 5 & 1 & 1 & & 2 & 18 & 4 & 1 & 3.92 & 0.83 \\
\hline 4 & $4-\mathrm{C}-2$ & Poa sandbergii & Sandberg's bluegrass & 6 & 4 & 8 & 2 & 10 & 5 & 4 & 5 & 11 & 12 & 2 & 5 & 6.17 & 1.00 \\
\hline 4 & $4-\mathrm{C}-2$ & Salsola kali & Russian thistle & & & & & & 1 & & 9 & & 2 & & & 1.00 & 0.25 \\
\hline 4 & $4-\mathrm{C}-2$ & Sisymbrium altissimum & $\begin{array}{l}\text { Jim Hill's } \\
\text { tumblemustard }\end{array}$ & & 17 & & & 5 & & & & 6 & 16 & & & 3.67 & 0.33 \\
\hline 4 & $4-\mathrm{C}-3$ & Agastache occidentalis & western horsemint & & & & & & 2 & & & & & 1 & 3 & 0.50 & 0.25 \\
\hline 4 & $4-\mathrm{C}-3$ & Agropyron spicatum & bluebunch wheatgrass & 12 & 8 & 8 & 10 & 16 & 6 & 12 & 12 & 3 & 2 & & & 7.42 & 0.83 \\
\hline 4 & $4-\mathrm{C}-3$ & Bare Ground & Bare Ground & 65 & 85 & 67 & 65 & 55 & 50 & 50 & 90 & 85 & 60 & 85 & 90 & 70.58 & 1.00 \\
\hline 4 & $4-\mathrm{C}-3$ & Bromus tectorum & cheatgrass & & & & & & 8 & & & & & 2 & & 0.83 & 0.17 \\
\hline 4 & $4-\mathrm{C}-3$ & Erigeron linearis & desert yellowdaisy & & & & & & & & 1 & & & & & 0.08 & 0.08 \\
\hline 4 & $4-\mathrm{C}-3$ & Eriogonum sphaerocephalum & rock buckwheat & & & & & & & & & & & 1 & & 0.08 & 0.08 \\
\hline 4 & $4-\mathrm{C}-3$ & Festuca idahoensis & Idaho fescue & 3 & 1 & 2 & 2 & 13 & 1 & 2 & & 1 & 2 & & & 2.25 & 0.75 \\
\hline 4 & $4-\mathrm{C}-3$ & Lactuca serriola & prickly lettuce & & & & & & & 1 & & & 1 & & & 0.17 & 0.17 \\
\hline 4 & $4-C-3$ & Litter & Litter & & 1 & 1 & 2 & 2 & 2 & & 2 & 1 & & & & 0.92 & 0.58 \\
\hline 4 & $4-\mathrm{C}-3$ & Phacelia hastata & whiteleaf scorpionweed & 2 & 4 & 2 & 2 & & 10 & 5 & 2 & 1 & 4 & 1 & 1 & 2.83 & 0.92 \\
\hline 4 & $4-\mathrm{C}-3$ & Poa sandbergii & Sandberg's bluegrass & 16 & 3 & 10 & 20 & 24 & 14 & 28 & 8 & 10 & 12 & 10 & 6 & 13.42 & 1.00 \\
\hline 4 & $4-\mathrm{C}-3$ & Salsola kali & Russian thistle & 4 & & & 4 & & 8 & & 1 & & 10 & & 4 & 2.58 & 0.50 \\
\hline 4 & $4-\mathrm{C}-3$ & Salvia dorrii & purple sage & & & & 1 & & & & & & & & & 0.08 & 0.08 \\
\hline 4 & $4-\mathrm{C}-3$ & Sisymbrium altissimum & $\begin{array}{l}\text { Jim Hill's } \\
\text { tumblemustard }\end{array}$ & & 6 & 10 & & & & & & & & & & 1.33 & 0.17 \\
\hline 4 & 4-CCF-1 & Achillea millefolium & yarrow & & & & & & & & 13 & & & 1 & & 1.17 & 0.17 \\
\hline 4 & 4-CCF-1 & Agropyron spicatum & bluebunch wheatgrass & 4 & 2 & 8 & 5 & 7 & & & 38 & 10 & & 5 & 7 & 7.17 & 0.75 \\
\hline 4 & 4-CCF-1 & Astragalus purshii & woolly-pod milkvetch & 1 & & & & & & & & & & & & 0.08 & 0.08 \\
\hline 4 & 4-CCF-1 & Balsamorhiza rosea & rosy balsamroot & & & 3 & 1 & 7 & 28 & 8 & 5 & & 2 & 5 & & 4.92 & 0.67 \\
\hline
\end{tabular}


Table C.1. (contd)

\begin{tabular}{|c|c|c|c|c|c|c|c|c|c|c|c|c|c|c|c|c|c|}
\hline $\begin{array}{l}\text { Reveg } \\
\text { Unit }\end{array}$ & Site Name & Genus/Species & Common Name & $1 \mathrm{~m}$ & $5 \mathrm{~m}$ & $\begin{array}{l}10 \\
\mathrm{~m}\end{array}$ & $\begin{array}{l}15 \\
\mathrm{~m}\end{array}$ & $\begin{array}{l}18 \\
\mathrm{~m}\end{array}$ & $\begin{array}{l}22 \\
\mathrm{~m}\end{array}$ & $\begin{array}{l}26 \\
\mathrm{~m}\end{array}$ & $\begin{array}{l}30 \\
\mathrm{~m}\end{array}$ & $\begin{array}{l}35 \\
\mathrm{~m}\end{array}$ & $\begin{array}{l}40 \\
\mathrm{~m}\end{array}$ & $\begin{array}{l}44 \\
\mathrm{~m}\end{array}$ & $\begin{array}{l}48 \\
\mathrm{~m}\end{array}$ & $\begin{array}{l}\text { Mean } \\
\text { Cover }\end{array}$ & Frequency \\
\hline 4 & 4-CCF-1 & Bare Ground & Bare Ground & 66 & 86 & 72 & 86 & 67 & 65 & 80 & 50 & 70 & 64 & 62 & 69 & 69.75 & 1.00 \\
\hline 4 & 4-CCF-1 & Bromus japonicus & Japanese brome & & & & & & & & 2 & & & 1 & & 0.25 & 0.17 \\
\hline 4 & 4-CCF-1 & Bromus tectorum & cheatgrass & 17 & 13 & 14 & 13 & 4 & 11 & 8 & 15 & 40 & 33 & 35 & 30 & 19.42 & 1.00 \\
\hline 4 & 4-CCF-1 & Chenopodium leptophyllum & slimleaf goosefoot & 4 & 1 & 1 & & & & & & 2 & & & 1 & 0.75 & 0.42 \\
\hline 4 & 4-CCF-1 & Crepis modocensis & low hawksbeard & & & & 1 & 6 & 7 & 6 & & & 3 & 8 & & 2.58 & 0.50 \\
\hline 4 & 4-CCF-1 & Litter & Litter & 7 & 5 & 1 & 2 & 2 & 2 & 1 & 3 & 3 & 6 & 2 & 4 & 3.17 & 1.00 \\
\hline 4 & 4-CCF-1 & Lomatium grayi & Gray's desertparsley & & & & 1 & & & & & & & & & 0.08 & 0.08 \\
\hline 4 & 4-CCF-1 & Phacelia hastata & whiteleaf scorpionweed & 1 & 1 & 1 & & 1 & & 1 & & & & 2 & 16 & 1.92 & 0.58 \\
\hline 4 & 4-CCF-1 & Phlox hoodii & Hood's phlox & & & & & & 1 & 4 & & & & 2 & & 0.58 & 0.25 \\
\hline 4 & 4-CCF-1 & Poa sandbergii & Sandberg's bluegrass & 40 & 30 & 39 & 27 & 35 & 29 & 29 & 18 & 9 & 7 & 22 & 36 & 26.75 & 1.00 \\
\hline 4 & 4-CCF-1 & Polygonum aviculare & doorweed & 15 & 6 & 19 & & & & & & & & & 7 & 3.92 & 0.33 \\
\hline 4 & 4-CCF-1 & Sisymbrium altissimum & $\begin{array}{l}\text { Jim Hill's } \\
\text { tumblemustard }\end{array}$ & & 23 & 1 & & & & & 1 & & 7 & 2 & & 2.83 & 0.42 \\
\hline 4 & 4-CCF-Berm & Agropyron spicatum & bluebunch wheatgrass & & & & 1 & & & & & & & & & 0.08 & 0.08 \\
\hline 4 & 4-CCF-Berm & Bare Ground & Bare Ground & 100 & 97 & 100 & 89 & 68 & 100 & & & & & & & 46.17 & 0.50 \\
\hline 4 & 4-CCF-Berm & Bromus tectorum & cheatgrass & & 7 & 1 & 15 & 17 & 8 & & & & & & & 4.00 & 0.42 \\
\hline 4 & 4-CCF-Berm & Litter & Litter & 1 & 2 & 5 & 8 & 2 & 1 & & & & & & & 1.58 & 0.50 \\
\hline 4 & 4-CCF-Berm & Phacelia hastata & whiteleaf scorpionweed & & 3 & & 19 & 52 & & & & & & & & 6.17 & 0.25 \\
\hline 4 & 4-CCF-Berm & Sisymbrium altissimum & $\begin{array}{l}\text { Jim Hill's } \\
\text { tumblemustard }\end{array}$ & & & & 3 & & & & & & & & & 0.25 & 0.08 \\
\hline 4 & 4-CCF-Road & Achillea millefolium & yarrow & 6 & & & & & & & & & & & & 0.50 & 0.08 \\
\hline 4 & 4-CCF-Road & Balsamorhiza rosea & rosy balsamroot & & 7 & 5 & 21 & & & & & & & & & 2.75 & 0.25 \\
\hline 4 & 4-CCF-Road & Bare Ground & Bare Ground & 87 & 90 & 90 & 85 & 53 & 88 & & & & & & & 41.08 & 0.50 \\
\hline 4 & 4-CCF-Road & Bromus japonicus & Japanese brome & 3 & & & & & & & & & & & & 0.25 & 0.08 \\
\hline 4 & 4-CCF-Road & Bromus tectorum & cheatgrass & 40 & 18 & 8 & 17 & 56 & 16 & & & & & & & 12.92 & 0.50 \\
\hline 4 & 4-CCF-Road & Crepis modocensis & low hawksbeard & & 1 & 3 & & & & & & & & & & 0.33 & 0.17 \\
\hline 4 & 4-CCF-Road & Litter & Litter & 5 & & 1 & & 7 & & & & & & & & 1.08 & 0.25 \\
\hline 4 & 4-CCF-Road & Lomatium grayi & Gray's desertparsley & 6 & & & 6 & 4 & 1 & & & & & & & 1.42 & 0.33 \\
\hline 4 & 4-CCF-Road & $\begin{array}{l}\text { Lygodesmia juncea (Pursh) D. } \\
\text { Don ex Hook. }\end{array}$ & rush skeletonplant & 7 & & 3 & & & & & & & & & & 0.83 & 0.17 \\
\hline 4 & 4-CCF-Road & Phacelia hastata & whiteleaf scorpionweed & & & & 4 & 4 & 8 & & & & & & & 1.33 & 0.25 \\
\hline
\end{tabular}


Table C.1. (contd)

\begin{tabular}{|c|c|c|c|c|c|c|c|c|c|c|c|c|c|c|c|c|c|}
\hline $\begin{array}{c}\text { Reveg } \\
\text { Unit }\end{array}$ & Site Name & Genus/Species & Common Name & $1 \mathrm{~m}$ & $5 \mathrm{~m}$ & $\begin{array}{l}10 \\
\mathrm{~m}\end{array}$ & $\begin{array}{l}15 \\
\mathrm{~m}\end{array}$ & $\begin{array}{l}18 \\
\mathrm{~m}\end{array}$ & $\begin{array}{l}22 \\
\mathrm{~m}\end{array}$ & $\begin{array}{l}26 \\
\mathrm{~m}\end{array}$ & $\begin{array}{l}30 \\
\mathrm{~m}\end{array}$ & $\begin{array}{l}35 \\
\mathrm{~m}\end{array}$ & $\begin{array}{l}40 \\
\mathrm{~m}\end{array}$ & $\begin{array}{l}44 \\
\mathrm{~m}\end{array}$ & $\begin{array}{l}48 \\
\mathrm{~m}\end{array}$ & $\begin{array}{l}\text { Mean } \\
\text { Cover }\end{array}$ & Frequency \\
\hline 4 & 4-CCF-Road & Poa sandbergii & Sandberg's bluegrass & 13 & 10 & 5 & & & & & & & & & & 2.33 & 0.25 \\
\hline 4 & 4-CCF-Road & Salsola kali & Russian thistle & & & & & & 4 & & & & & & & 0.33 & 0.08 \\
\hline 4 & 4-CCF-Road & Sisymbrium altissimum & $\begin{array}{l}\text { Jim Hill's } \\
\text { tumblemustard }\end{array}$ & & & & 3 & & & & & & & & & 0.25 & 0.08 \\
\hline 5 & $5-\mathrm{OB}-1$ & Agropyron spicatum & bluebunch wheatgrass & 2 & 1 & 2 & 2 & 1 & 1 & & 2 & & 5 & 2 & 2 & 1.67 & 0.83 \\
\hline 5 & $5-\mathrm{OB}-1$ & Bare Ground & Bare Ground & 95 & 97 & 90 & 97 & 98 & 93 & 94 & 91 & 97 & 90 & 87 & 88 & 93.08 & 1.00 \\
\hline 5 & $5-\mathrm{OB}-1$ & Bromus tectorum & cheatgrass & & & & & & 1 & & & & & & & 0.08 & 0.08 \\
\hline 5 & $5-\mathrm{OB}-1$ & Chenopodium leptophyllum & slimleaf goosefoot & & & & & & & & & 1 & & & & 0.08 & 0.08 \\
\hline 5 & $5-\mathrm{OB}-1$ & Litter & Litter & & & 2 & 1 & & 1 & 2 & & 2 & 1 & 2 & 2 & 1.08 & 0.67 \\
\hline 5 & $5-\mathrm{OB}-1$ & Phacelia hastata & whiteleaf scorpionweed & 2 & & 2 & & & & 6 & 2 & & & 4 & 2 & 1.50 & 0.50 \\
\hline 5 & $5-\mathrm{OB}-1$ & Poa sandbergii & Sandberg's bluegrass & 3 & 1 & 2 & & 1 & 2 & & 1 & 1 & 2 & 2 & 4 & 1.58 & 0.83 \\
\hline 5 & $5-\mathrm{OB}-2$ & Agropyron spicatum & bluebunch wheatgrass & 4 & 1 & & & & & 1 & & & & 1 & 6 & 1.08 & 0.42 \\
\hline 5 & $5-\mathrm{OB}-2$ & Bare Ground & Bare Ground & 89 & 96 & 97 & 94 & 93 & 98 & 96 & 87 & 92 & 90 & 88 & 91 & 92.58 & 1.00 \\
\hline 5 & $5-\mathrm{OB}-2$ & Litter & Litter & 1 & 1 & 1 & 1 & 1 & & 2 & 2 & 1 & 3 & 2 & 4 & 1.58 & 0.92 \\
\hline 5 & $5-\mathrm{OB}-2$ & Phacelia hastata & whiteleaf scorpionweed & 1 & & & & & & & & & 2 & & & 0.25 & 0.17 \\
\hline 5 & $5-\mathrm{OB}-2$ & Poa sandbergii & Sandberg's bluegrass & 4 & 2 & 2 & 5 & 6 & 2 & & 11 & 7 & 7 & 9 & 3 & 4.83 & 0.92 \\
\hline 5 & $5-\mathrm{OB}-2$ & Salsola kali & Russian thistle & 1 & & & & & & 1 & & & & & & 0.17 & 0.17 \\
\hline 6 & $6-6635-X$ & Agastache occidentalis & western horsemint & & & & 1 & & & & & & & & & 0.08 & 0.08 \\
\hline 6 & $6-6635-X$ & Agropyron spicatum & bluebunch wheatgrass & 10 & 16 & 12 & 6 & 16 & 2 & 5 & 11 & 15 & 1 & 27 & 9 & 10.83 & 1.00 \\
\hline 6 & $6-6635-X$ & Astragalus purshii & woolly-pod milkvetch & & 1 & & & & & & & & & & & 0.08 & 0.08 \\
\hline 6 & $6-6635-X$ & Bare Ground & Bare Ground & 57 & 73 & 34 & 74 & 54 & 54 & 66 & 61 & 64 & 4 & 87 & 71 & 58.25 & 1.00 \\
\hline 6 & $6-6635-X$ & Bromus tectorum & cheatgrass & 2 & 1 & & 1 & & & 5 & 9 & 10 & 1 & & & 2.42 & 0.58 \\
\hline 6 & 6-6635-X & Chenopodium leptophyllum & slimleaf goosefoot & & 1 & 3 & & & & 4 & 1 & 1 & 1 & & & 0.92 & 0.50 \\
\hline 6 & $6-6635-\mathrm{X}$ & Descurainia pinnata & western tansymustard & & & & & & & & & & 1 & & & 0.08 & 0.08 \\
\hline 6 & $6-6635-\mathrm{X}$ & Descurainia sophia & flixweed & & & & & & & & & & & & 1 & 0.08 & 0.08 \\
\hline 6 & $6-6635-X$ & Festuca idahoensis & Idaho fescue & & & & & & & & & & 1 & & & 0.08 & 0.08 \\
\hline 6 & $6-6635-\mathrm{X}$ & Litter & Litter & 5 & 3 & 1 & 2 & 3 & 1 & 3 & 1 & 5 & 80 & 6 & 0 & 9.17 & 0.92 \\
\hline 6 & $6-6635-X$ & Lomatium grayi & Gray's desertparsley & & & & & 2 & & & & & & & & 0.17 & 0.08 \\
\hline 6 & $6-6635-X$ & Phacelia hastata & whiteleaf scorpionweed & 6 & 2 & 1 & 2 & 1 & & 5 & 10 & 14 & 1 & & 4 & 3.83 & 0.83 \\
\hline 6 & $6-6635-X$ & Poa sandbergii & Sandberg's bluegrass & & & & & & & & & & 4 & & & 0.33 & 0.08 \\
\hline 6 & $6-6635-X$ & Polygonum aviculare & doorweed & 30 & 42 & 64 & 30 & 50 & 60 & 17 & 35 & 30 & 24 & 30 & 32 & 37.00 & 1.00 \\
\hline
\end{tabular}


Table C.1. (contd)

\begin{tabular}{|c|c|c|c|c|c|c|c|c|c|c|c|c|c|c|c|c|c|}
\hline $\begin{array}{l}\text { Reveg } \\
\text { Unit }\end{array}$ & Site Name & Genus/Species & Common Name & $1 \mathrm{~m}$ & $5 \mathrm{~m}$ & $\begin{array}{l}10 \\
\mathrm{~m}\end{array}$ & $\begin{array}{l}15 \\
\mathrm{~m}\end{array}$ & $\begin{array}{l}18 \\
\mathrm{~m}\end{array}$ & $\begin{array}{l}22 \\
\mathrm{~m}\end{array}$ & $\begin{array}{l}26 \\
\mathrm{~m}\end{array}$ & $\begin{array}{l}30 \\
\mathrm{~m}\end{array}$ & $\begin{array}{l}35 \\
\mathrm{~m}\end{array}$ & $\begin{array}{l}40 \\
\mathrm{~m}\end{array}$ & $\begin{array}{l}44 \\
\mathrm{~m}\end{array}$ & $\begin{array}{l}48 \\
\mathrm{~m}\end{array}$ & $\begin{array}{l}\text { Mean } \\
\text { Cover }\end{array}$ & Frequency \\
\hline 6 & $6-6635-X$ & Salsola kali & Russian thistle & & & & 1 & & & & & & & & & 0.08 & 0.08 \\
\hline 6 & $6-6635-\mathrm{X}$ & Sisymbrium altissimum & $\begin{array}{l}\text { Jim Hill's } \\
\text { tumblemustard }\end{array}$ & 14 & 9 & 4 & 36 & 37 & 7 & 57 & 32 & 50 & 35 & 26 & 17 & 27.00 & 1.00 \\
\hline 6 & $6-6636-1$ & Achillea millefolium & yarrow & & & & & 1 & & & & & & & & 0.08 & 0.08 \\
\hline 6 & $6-6636-1$ & Agropyron spicatum & bluebunch wheatgrass & 1 & 2 & 5 & 3 & 2 & 2 & 5 & 12 & 16 & 5 & 7 & 4 & 5.33 & 1.00 \\
\hline 6 & $6-6636-1$ & Astragalus purshii & woolly-pod milkvetch & & & & & & & & & 1 & & 1 & & 0.17 & 0.17 \\
\hline 6 & $6-6636-1$ & Balsamorhiza rosea & rosy balsamroot & 1 & 3 & 1 & & & & & & & & & & 0.42 & 0.25 \\
\hline 6 & $6-6636-1$ & Bare Ground & Bare Ground & 76 & 76 & 74 & 73 & 80 & 85 & 93 & 80 & 75 & 91 & 73 & 84 & 80.00 & 1.00 \\
\hline 6 & 6-6636-1 & Bromus tectorum & cheatgrass & 1 & 4 & & & & 4 & 7 & & & & & 1 & 1.42 & 0.42 \\
\hline 6 & $6-6636-1$ & Crepis modocensis & low hawksbeard & 6 & 7 & 1 & 10 & 1 & 1 & & & & 2 & 10 & 1 & 3.25 & 0.75 \\
\hline 6 & $6-6636-1$ & Descurainia pinnata & western tansymustard & & & & & & 3 & & & & & & & 0.25 & 0.08 \\
\hline 6 & $6-6636-1$ & Erigeron linearis & desert yellowdaisy & & & & & & & & & 1 & & & & 0.08 & 0.08 \\
\hline 6 & $6-6636-1$ & Eriogonum sphaerocephalum & rock buckwheat & & & & & & & & & & & 1 & & 0.08 & 0.08 \\
\hline 6 & $6-6636-1$ & Haplopappus stenophylla & narrowleaf goldenweed & & & & & & & & & & 1 & & & 0.08 & 0.08 \\
\hline 6 & $6-6636-1$ & Litter & Litter & 1 & 1 & 2 & 1 & 1 & 8 & & & & & 1 & & 1.25 & 0.58 \\
\hline 6 & $6-6636-1$ & Lomatium grayi & Gray's desertparsley & & & 6 & & & 26 & & & & & & & 2.67 & 0.17 \\
\hline 6 & $6-6636-1$ & Phacelia hastata & whiteleaf scorpionweed & 5 & 2 & 2 & 4 & 2 & 6 & 2 & & 1 & 1 & & 1 & 2.17 & 0.83 \\
\hline 6 & $6-6636-1$ & Phlox hoodii & Hood's phlox & & 1 & & & & & & & & & 5 & 8 & 1.17 & 0.25 \\
\hline 6 & 6-6636-1 & Poa sandbergii & Sandberg's bluegrass & 6 & 1 & & 2 & & & & & & & 13 & 1 & 1.92 & 0.42 \\
\hline 6 & $6-6636-1$ & Polygonum aviculare & doorweed & 11 & 20 & 20 & 2 & 2 & 1 & 12 & 11 & 15 & 11 & & 7 & 9.33 & 0.92 \\
\hline 6 & $6-6636-1$ & Ribes aureum & golden currant & & & & & & 1 & & & & & & & 0.08 & 0.08 \\
\hline 6 & $6-6636-1$ & Sisymbrium altissimum & $\begin{array}{l}\text { Jim Hill's } \\
\text { tumblemustard }\end{array}$ & 10 & & & & 28 & & & & & & & & 3.17 & 0.17 \\
\hline 6 & $6-6636-1$ & Sitanion hystrix & bottlebrush grass & 1 & & & & & 1 & 1 & 1 & 1 & & & 1 & 0.50 & 0.50 \\
\hline 7 & 7-PH-X & Achillea millefolium & yarrow & 7 & 10 & 2 & 2 & 5 & 40 & & & 5 & 2 & 2 & 2 & 6.42 & 0.83 \\
\hline 7 & 7-PH-X & Agastache occidentalis & western horsemint & & & & & 3 & & & & & & & & 0.25 & 0.08 \\
\hline 7 & 7-PH-X & Agropyron spicatum & bluebunch wheatgrass & 4 & 24 & 2 & 2 & & & 1 & 1 & 1 & 2 & & & 3.08 & 0.67 \\
\hline 7 & 7-PH-X & Artemisia tridentata & big sagebrush & & & & & & & & & 1 & 1 & & 1 & 0.25 & 0.25 \\
\hline 7 & 7-PH-X & Bare Ground & Bare Ground & 21 & 2 & 44 & 29 & 3 & 17 & 96 & 97 & 96 & 33 & 23 & 67 & 44.00 & 1.00 \\
\hline 7 & 7-PH-X & Bromus tectorum & cheatgrass & 38 & 18 & 48 & 52 & 52 & 30 & 6 & 4 & 6 & 65 & 70 & 52 & 36.75 & 1.00 \\
\hline 7 & 7-PH-X & Chrysothamnus nauseosus & gray rabbitbrush & & & & & & & 4 & & & & & & 0.33 & 0.08 \\
\hline 7 & 7-PH-X & Descurainia pinnata & western tansymustard & & & & & & & & & & & 27 & 7 & 2.83 & 0.17 \\
\hline
\end{tabular}


Table C.1. (contd)

\begin{tabular}{|c|c|c|c|c|c|c|c|c|c|c|c|c|c|c|c|c|c|}
\hline $\begin{array}{c}\text { Reveg } \\
\text { Unit }\end{array}$ & Site Name & Genus/Species & Common Name & $1 \mathrm{~m}$ & $5 \mathrm{~m}$ & $\begin{array}{l}10 \\
\mathrm{~m}\end{array}$ & $\begin{array}{l}15 \\
\mathrm{~m}\end{array}$ & $\begin{array}{l}18 \\
\mathrm{~m}\end{array}$ & $\begin{array}{c}22 \\
\mathrm{~m}\end{array}$ & $\begin{array}{c}26 \\
\mathrm{~m}\end{array}$ & $\begin{array}{l}30 \\
\mathrm{~m}\end{array}$ & $\begin{array}{l}35 \\
\mathrm{~m}\end{array}$ & $\begin{array}{l}40 \\
\mathrm{~m}\end{array}$ & $\begin{array}{c}44 \\
\mathrm{~m}\end{array}$ & $\begin{array}{c}48 \\
\mathrm{~m}\end{array}$ & $\begin{array}{l}\text { Mean } \\
\text { Cover }\end{array}$ & Frequency \\
\hline 7 & 7-PH-X & Elymus cinereus & giant wildrye & & & 1 & & & & 1 & & & & & & 0.17 & 0.17 \\
\hline 7 & 7-PH-X & Litter & Litter & 14 & 78 & 8 & 5 & 15 & 9 & 4 & 3 & 4 & 13 & 2 & 1 & 13.00 & 1.00 \\
\hline 7 & 7-PH-X & Lomatium sp. & & 5 & & 2 & 2 & 6 & & & & & 6 & & 1 & 1.83 & 0.50 \\
\hline 7 & 7-PH-X & Phacelia hastata & whiteleaf scorpionweed & & 4 & 1 & & & & 1 & & & & & & 0.50 & 0.25 \\
\hline 7 & 7-PH-X & Poa bulbosa & bulbous bluegrass & & 14 & & & & & & & & & & & 1.17 & 0.08 \\
\hline 7 & 7-PH-X & Poa sandbergii & Sandberg's bluegrass & 2 & & 1 & 4 & 16 & 16 & 4 & 1 & 1 & & & 2 & 3.92 & 0.75 \\
\hline 7 & 7-PH-X & Ribes aureum & golden currant & & 2 & & & & & & & & & & & 0.17 & 0.08 \\
\hline 7 & 7-PH-X & Sisymbrium altissimum & $\begin{array}{l}\text { Jim Hill's } \\
\text { tumblemustard }\end{array}$ & 22 & 12 & 7 & 7 & 45 & 14 & & & & 9 & 12 & & 10.67 & 0.67 \\
\hline 7 & 7-PH-X & Sitanion hystrix & bottlebrush grass & & & 1 & & & & & & 1 & 1 & & 1 & 0.33 & 0.33 \\
\hline
\end{tabular}


Table C.2. Density of Plant Species Counted Within Modified Whittaker 100- $\mathrm{m}^{2}$ Monitoring Plots

\begin{tabular}{|c|c|c|c|c|c|c|c|c|c|c|c|c|c|c|c|c|}
\hline $\begin{array}{c}\text { Reveg } \\
\text { Unit }\end{array}$ & Site Name & Genus/Species & Common Name & $1 \mathrm{~m}$ & $5 \mathrm{~m}$ & $\begin{array}{l}10 \\
\mathrm{~m}\end{array}$ & $\begin{array}{l}15 \\
\mathrm{~m}\end{array}$ & $\begin{array}{l}18 \\
\mathrm{~m}\end{array}$ & $\begin{array}{l}22 \\
\mathrm{~m}\end{array}$ & $\begin{array}{l}26 \\
\mathrm{~m}\end{array}$ & $\begin{array}{l}30 \\
\mathrm{~m}\end{array}$ & $\begin{array}{l}35 \\
\mathrm{~m}\end{array}$ & $\begin{array}{l}40 \\
\mathrm{~m}\end{array}$ & $\begin{array}{l}44 \\
\mathrm{~m}\end{array}$ & $\begin{array}{l}48 \\
\mathrm{~m}\end{array}$ & $\begin{array}{c}\text { Mean } \\
\text { Density }\end{array}$ \\
\hline 0 & 1200-REF-1 & Achillea millefolium & yarrow & 5 & 4 & 4 & 15 & 8 & 2 & 1 & 7 & & 2 & & & 4.00 \\
\hline 0 & 1200-REF-1 & Agropyron spicatum & bluebunch wheatgrass & & 7 & 4 & 15 & 6 & 3 & 4 & 5 & 3 & 4 & 5 & 4 & 5.00 \\
\hline 0 & 1200-REF-1 & Astragalus purshii & woolly-pod milkvetch & & & 1 & & & & & & 1 & & & & 0.17 \\
\hline 0 & 1200-REF-1 & Bromus tectorum & cheatgrass & 3 & 1 & & & & & & & & & & & 0.33 \\
\hline 0 & 1200-REF-1 & Calochortus macrocarpus & sagebrush mariposa lily & & & & & 1 & & & 1 & & & & & 0.17 \\
\hline 0 & 1200-REF-1 & Crepis atribarba & slender hawksbeard & & 2 & & 1 & 1 & & & & & 3 & & & 0.58 \\
\hline 0 & 1200-REF-1 & Erigeron filifolius & threadleaf fleabane & 4 & & 2 & 2 & & 2 & 11 & & & & 1 & 12 & 2.83 \\
\hline 0 & 1200-REF-1 & Holosteum umbellatum & jagged chickweed & & & & 30 & 35 & 10 & & & & & & & 6.25 \\
\hline 0 & 1200-REF-1 & Lomatium macrocarpum & bigseed desertparsley & & 1 & 2 & & & & & 1 & & & 1 & 1 & 0.50 \\
\hline 0 & 1200-REF-1 & Lupinus sericeus & silky lupine & & & & 1 & & & & & & & & & 0.08 \\
\hline 0 & 1200-REF-1 & Phlox longifolia & longleaf phlox & & & & & & & 1 & & & 1 & & 1 & 0.25 \\
\hline 0 & 1200-REF-1 & Poa sandbergii & Sandberg's bluegrass & 33 & 16 & 32 & 13 & 11 & 19 & 10 & 13 & 26 & 14 & 26 & 17 & 19.17 \\
\hline 0 & RR-1 & Achillea millefolium & yarrow & 2 & & & & & 1 & & & & 2 & & & 0.42 \\
\hline 0 & RR-1 & Agropyron spicatum & bluebunch wheatgrass & 2 & 3 & 1 & & 3 & & 3 & & 2 & & 2 & & 1.33 \\
\hline 0 & RR-1 & Antennaria dimorpha & low pussytoes & & & & & 3 & 2 & & & 1 & 5 & 1 & 1 & 1.08 \\
\hline 0 & RR-1 & Astragalus purshii & woolly-pod milkvetch & & 1 & 2 & 1 & 1 & & & 2 & & & & & 0.58 \\
\hline 0 & RR-1 & Balsamorhiza rosea & rosy balsamroot & 3 & 1 & 6 & 2 & 1 & 1 & 2 & 18 & 2 & 7 & 1 & 4 & 4.00 \\
\hline 0 & RR-1 & Bromus tectorum & cheatgrass & & & & & & 1 & 1 & 1 & 1 & & & & 0.33 \\
\hline 0 & RR-1 & Crepis modocensis & low hawksbeard & 5 & 10 & 7 & & 7 & 3 & 4 & 3 & 7 & 4 & 5 & 10 & 5.42 \\
\hline 0 & RR-1 & Erigeron linearis & desert yellowdaisy & & & & & 1 & & & & & & 1 & & 0.17 \\
\hline 0 & RR-1 & Eriogonum sphaerocephalum & rock buckwheat & 1 & & & 1 & 3 & & 2 & & & & & & 0.58 \\
\hline 0 & RR-1 & Eriogonum thymoides & thymeleaf buckwheat & & 1 & & 2 & 1 & 1 & 1 & & & & & 1 & 0.58 \\
\hline 0 & RR-1 & Festuca idahoensis & Idaho fescue & 5 & & & & & 1 & & 2 & 1 & 5 & & 3 & 1.42 \\
\hline 0 & RR-1 & Haplopappus stenophylla & narrowleaf goldenweed & & 1 & & 2 & & 1 & & & & 1 & & & 0.42 \\
\hline 0 & RR-1 & Lupinus sericeus & silky lupine & 1 & & & & & & & & 1 & & 3 & 1 & 0.50 \\
\hline 0 & RR-1 & Phlox hoodii & Hood's phlox & 5 & 9 & 8 & 10 & 4 & 12 & 8 & 9 & 4 & 1 & 3 & 2 & 6.25 \\
\hline 0 & RR-1 & Phoenicaulis cheiranthoides & daggerpod & & & & & & & & 1 & & & & & 0.08 \\
\hline 0 & RR-1 & Poa sandbergii & Sandberg's bluegrass & 49 & 32 & 34 & 39 & 44 & 42 & 58 & 33 & 40 & 25 & 13 & 53 & 38.50 \\
\hline 0 & RR-1 & Sitanion hystrix & bottlebrush grass & & & 2 & & & & & & & & & & 0.17 \\
\hline 1 & 1-RS-1 & Kochia scoparia & summer cypress & & & 2 & & & & & & 2 & & & & 0.33 \\
\hline
\end{tabular}


Table C.2. (contd)

\begin{tabular}{|c|c|c|c|c|c|c|c|c|c|c|c|c|c|c|c|c|}
\hline $\begin{array}{c}\text { Reveg } \\
\text { Unit }\end{array}$ & Site Name & Genus/Species & Common Name & $1 \mathrm{~m}$ & $5 \mathrm{~m}$ & $\begin{array}{l}10 \\
\mathrm{~m}\end{array}$ & $\begin{array}{l}15 \\
\mathrm{~m}\end{array}$ & $\begin{array}{l}18 \\
\mathrm{~m}\end{array}$ & $\begin{array}{l}22 \\
\mathrm{~m}\end{array}$ & $\begin{array}{l}26 \\
\mathrm{~m}\end{array}$ & $\begin{array}{l}30 \\
\mathrm{~m}\end{array}$ & $\begin{array}{l}35 \\
\mathrm{~m}\end{array}$ & $\begin{array}{l}40 \\
\mathrm{~m}\end{array}$ & $\begin{array}{l}44 \\
\mathrm{~m}\end{array}$ & $\begin{array}{l}48 \\
\mathrm{~m}\end{array}$ & $\begin{array}{c}\text { Mean } \\
\text { Density }\end{array}$ \\
\hline 1 & 1-RS-1 & Salsola kali & Russian thistle & & 2 & & 18 & & 2 & 3 & 3 & 6 & 6 & 3 & 1 & 3.67 \\
\hline 1 & 1-RS-1 & Sitanion hystrix & bottlebrush grass & & & & 16 & & & & & 7 & 13 & & & 3.00 \\
\hline 1 & 1-RS-2 & Artemisia tridentata & big sagebrush & & & & & & & & & 1 & & & & 0.08 \\
\hline 1 & 1-RS-2 & Bromus tectorum & cheatgrass & & 11 & & & & & & & & & & & 0.92 \\
\hline 1 & 1-RS-2 & Distichlis stricta & alkali saltgrass & 2 & 16 & 12 & 10 & & 1 & 13 & 9 & 2 & & & & 5.42 \\
\hline 1 & 1-RS-2 & Elymus cinereus & giant wildrye & & & & 4 & & & & & & & & & 0.33 \\
\hline 1 & 1-RS-2 & Salsola kali & Russian thistle & 13 & 54 & 12 & 6 & & 3 & 8 & 9 & 4 & 9 & 2 & 3 & 10.25 \\
\hline 1 & $1-\mathrm{RS}-2$ & Sitanion hystrix & bottlebrush grass & 34 & & 15 & 7 & & & & 35 & 34 & 1 & 1 & 6 & 11.08 \\
\hline 1 & 1-RS-3 & Achillea millefolium & yarrow & & & & & & & & & 2 & & & & 0.17 \\
\hline 1 & 1-RS-3 & Artemisia tridentata & big sagebrush & & 1 & & & & & & & & & & & 0.08 \\
\hline 1 & 1-RS-3 & Bromus tectorum & cheatgrass & & & & & & & 1 & & 9 & 3 & 5 & 5 & 1.92 \\
\hline 1 & 1-RS-3 & Distichlis stricta & alkali saltgrass & 4 & & 1 & & & & 9 & & & & 1 & & 1.25 \\
\hline 1 & 1-RS-3 & Elymus cinereus & giant wildrye & & & & & 1 & & & & & & & & 0.08 \\
\hline 1 & $1-\mathrm{RS}-3$ & Grayia spinosa & spiny hopsage & & & & & & & & & 1 & & & & 0.08 \\
\hline 1 & 1-RS-3 & Kochia scoparia & summer cypress & & 1 & & & & & & & & & & & 0.08 \\
\hline 1 & 1-RS-3 & Poa sandbergii & Sandberg's bluegrass & & & & & & 1 & & & & 5 & 34 & 18 & 4.83 \\
\hline 1 & 1-RS-3 & Salsola kali & Russian thistle & 17 & 11 & 19 & 6 & 5 & 1 & 7 & 29 & 94 & 14 & 16 & 12 & 19.25 \\
\hline 1 & 1-RS-3 & Sisymbrium altissimum & Jim Hill's tumblemustard & & & & & 1 & & 2 & 4 & 2 & & 1 & 1 & 0.92 \\
\hline 1 & 1-RS-3 & Sitanion hystrix & bottlebrush grass & 28 & 19 & 15 & 26 & 23 & 11 & 7 & 9 & 9 & 18 & 16 & 35 & 18.00 \\
\hline 2 & $2-109-1$ & Achillea millefolium & yarrow & & & & & & & & & & & 1 & 1 & 0.17 \\
\hline 2 & $2-109-1$ & Achnatherum thurberianum & thurber's needlegrass & 1 & 3 & 2 & & & 3 & 4 & & & 2 & & 3 & 1.50 \\
\hline 2 & $2-109-1$ & Agropyron spicatum & bluebunch wheatgrass & 2 & 4 & 10 & 2 & & & 0 & 1 & 14 & & & 3 & 3.00 \\
\hline 2 & $2-109-1$ & Amsinckia tessellata & devil's lettuce & 2 & 1 & & & & & & & 6 & 4 & & & 1.08 \\
\hline 2 & $2-109-1$ & Bromus tectorum & cheatgrass & 73 & 47 & 36 & 150 & 127 & 210 & 490 & 870 & 7 & 310 & 400 & 230 & 245.83 \\
\hline 2 & $2-109-1$ & Crepis atribarba & slender hawksbeard & & & & 1 & & 3 & & 3 & 1 & & & & 0.67 \\
\hline 2 & $2-109-1$ & Erodium cicutarium & storksbill & & & & & & 1 & & & & 2 & 1 & & 0.33 \\
\hline 2 & $2-109-1$ & Lactuca serriola & prickly lettuce & & & 1 & 2 & & 2 & & 9 & 5 & 8 & & 2 & 2.42 \\
\hline 2 & $2-109-1$ & Linum perenne & wild blueflax & & & & & & & 1 & & & & & & 0.08 \\
\hline 2 & $2-109-1$ & Lupinus sericeus & silky lupine & & & 2 & 1 & 2 & 1 & 3 & 2 & 1 & & & 1 & 1.08 \\
\hline 2 & $2-109-1$ & Machaeranthera canescens & hoary aster & & & & 2 & & & & & & & & & 0.17 \\
\hline
\end{tabular}


Table C.2. (contd)

\begin{tabular}{|c|c|c|c|c|c|c|c|c|c|c|c|c|c|c|c|c|}
\hline $\begin{array}{c}\text { Reveg } \\
\text { Unit }\end{array}$ & Site Name & Genus/Species & Common Name & $1 \mathrm{~m}$ & $5 \mathrm{~m}$ & $\begin{array}{l}10 \\
\mathrm{~m}\end{array}$ & $\begin{array}{l}15 \\
\mathrm{~m}\end{array}$ & $\begin{array}{l}18 \\
\mathrm{~m}\end{array}$ & $\begin{array}{l}22 \\
\mathrm{~m}\end{array}$ & $\begin{array}{c}26 \\
\mathrm{~m}\end{array}$ & $\begin{array}{l}30 \\
\mathrm{~m}\end{array}$ & $\begin{array}{l}35 \\
\mathrm{~m}\end{array}$ & $\begin{array}{l}40 \\
\mathrm{~m}\end{array}$ & $\begin{array}{l}44 \\
\mathrm{~m}\end{array}$ & $\begin{array}{l}48 \\
\mathrm{~m}\end{array}$ & $\begin{array}{c}\text { Mean } \\
\text { Density }\end{array}$ \\
\hline 2 & $2-109-1$ & Poa bulbosa & bulbous bluegrass & & 4 & 1 & 9 & 12 & 27 & 9 & 34 & & 1 & 5 & 1 & 8.58 \\
\hline 2 & $2-109-1$ & Poa sandbergii & Sandberg's bluegrass & 2 & & & & & & & 1 & 16 & & & 1 & 1.67 \\
\hline 2 & $2-109-1$ & Sisymbrium altissimum & Jim Hill's tumblemustard & 9 & 4 & 6 & & 6 & 1 & & & 13 & & 1 & & 3.33 \\
\hline 2 & $2-109-1$ & Sitanion hystrix & bottlebrush grass & & & & 2 & & & & & & & & & 0.17 \\
\hline 2 & $2-109-2$ & Agropyron spicatum & bluebunch wheatgrass & 12 & 14 & 5 & 5 & 10 & 9 & 3 & & 28 & 32 & 12 & 13 & 11.92 \\
\hline 2 & $2-109-2$ & Astragalus caricinus & buckwheat milkvetch & & 4 & 4 & 4 & 1 & 3 & 1 & & 1 & & & & 1.50 \\
\hline 2 & $2-109-2$ & Bromus tectorum & cheatgrass & 34 & 16 & 9 & 13 & 49 & 31 & 54 & 124 & 24 & 2 & 2 & 3 & 30.08 \\
\hline 2 & $2-109-2$ & Crepis atribarba & slender hawksbeard & 1 & & 3 & 1 & 1 & 2 & & & 1 & 2 & 1 & 2 & 1.17 \\
\hline 2 & $2-109-2$ & Erigeron filifolius & threadleaf fleabane & & & & & 1 & & & & & & & & 0.08 \\
\hline 2 & $2-109-2$ & Lupinus sericeus & silky lupine & & & & & & & & & & & & & 0.00 \\
\hline 2 & $2-109-2$ & Phlox longifolia & longleaf phlox & 1 & 12 & 2 & & & 20 & 8 & 8 & 1 & 5 & 13 & 7 & 6.42 \\
\hline 2 & $2-109-2$ & Poa bulbosa & bulbous bluegrass & 11 & 2 & 2 & 4 & 24 & 9 & 12 & 47 & & 2 & 2 & 3 & 9.83 \\
\hline 2 & $2-109-2$ & Poa sandbergii & Sandberg's bluegrass & 9 & & 4 & 1 & 22 & 15 & 12 & 3 & 16 & 7 & 2 & 13 & 8.67 \\
\hline 2 & $2-109-2$ & Stipa comata & needle-and-thread grass & 12 & & 1 & & 2 & 10 & & 2 & & & & & 2.25 \\
\hline 2 & $2-109-2$ & Tragopogon dubius & Yellow salsify & & & & 1 & & & & & & & & & 0.08 \\
\hline 2 & $2-109-3$ & Achillea millefolium & yarrow & 1 & & 2 & & & 1 & 4 & 4 & 3 & & & & 1.25 \\
\hline 2 & $2-109-3$ & Agropyron spicatum & bluebunch wheatgrass & 26 & 14 & 4 & 4 & 8 & 4 & 9 & 10 & 8 & 5 & 3 & 16 & 9.25 \\
\hline 2 & $2-109-3$ & Artemisia tridentata & big sagebrush & & & & & & 1 & & & & & & & 0.08 \\
\hline 2 & $2-109-3$ & Astragalus caricinus & buckwheat milkvetch & & & & & & & 2 & & 2 & & & & 0.33 \\
\hline 2 & $2-109-3$ & Bromus tectorum & cheatgrass & & & 15 & 7 & 1 & 7 & & & 1 & & 1 & 1 & 2.75 \\
\hline 2 & $2-109-3$ & Crepis atribarba & slender hawksbeard & 5 & 6 & 1 & 10 & 5 & & & & 1 & & 3 & 5 & 3.00 \\
\hline 2 & $2-109-3$ & Erigeron filifolius & threadleaf fleabane & & 1 & & & & 3 & & & & & & 3 & 0.58 \\
\hline 2 & $2-109-3$ & Lactuca serriola & prickly lettuce & & & & & & 1 & & & & & & & 0.08 \\
\hline 2 & $2-109-3$ & Lomatium macrocarpum & bigseed biscuitroot & & & & & & & & & & 1 & & 1 & 0.17 \\
\hline 2 & $2-109-3$ & Lupinus sericeus & silky lupine & 1 & 2 & 1 & & 2 & & & & 1 & & & & 0.58 \\
\hline 2 & $2-109-3$ & Phlox longifolia & longleaf phlox & & & & & 3 & 5 & & & 2 & & & 1 & 0.92 \\
\hline 2 & $2-109-3$ & Poa bulbosa & bulbous bluegrass & 11 & 13 & 47 & 28 & 18 & 2 & & 10 & & & & 1 & 10.83 \\
\hline 2 & $2-109-3$ & Poa sandbergii & Sandberg's bluegrass & 5 & 12 & 4 & 7 & 14 & 6 & 15 & 12 & & & & 13 & 7.33 \\
\hline 2 & $2-109-3$ & Sisymbrium altissimum & Jim Hill's tumblemustard & & & 1 & & & & & & & & & & 0.08 \\
\hline 2 & $2-109-3$ & Stipa comata & needle-and-thread grass & & & & & & 1 & & & & & & & 0.08 \\
\hline
\end{tabular}


Table C.2. (contd)

\begin{tabular}{|c|c|c|c|c|c|c|c|c|c|c|c|c|c|c|c|c|}
\hline $\begin{array}{c}\text { Reveg } \\
\text { Unit }\end{array}$ & Site Name & Genus/Species & Common Name & $1 \mathrm{~m}$ & $5 \mathrm{~m}$ & $\begin{array}{l}10 \\
\mathrm{~m}\end{array}$ & $\begin{array}{l}15 \\
\mathrm{~m}\end{array}$ & $\begin{array}{l}18 \\
\mathrm{~m}\end{array}$ & $\begin{array}{l}22 \\
\mathrm{~m}\end{array}$ & $\begin{array}{c}26 \\
\mathrm{~m}\end{array}$ & $\begin{array}{l}30 \\
\mathrm{~m}\end{array}$ & $\begin{array}{l}35 \\
\mathrm{~m}\end{array}$ & $\begin{array}{l}40 \\
\mathrm{~m}\end{array}$ & $\begin{array}{c}44 \\
\mathrm{~m}\end{array}$ & $\begin{array}{c}48 \\
\mathrm{~m}\end{array}$ & $\begin{array}{c}\text { Mean } \\
\text { Density }\end{array}$ \\
\hline 2 & $2-138-1$ & Agropyron spicatum & bluebunch wheatgrass & 3 & 7 & 8 & 14 & & & & & & & & & 2.67 \\
\hline 2 & $2-138-1$ & Amsinckia tessellata & devil's lettuce & 2 & 2 & & 1 & & & & & & & & & 0.42 \\
\hline 2 & $2-138-1$ & Artemisia tridentata & big sagebrush & 4 & 3 & 3 & 10 & & & & & & & & & 1.67 \\
\hline 2 & $2-138-1$ & Bromus tectorum & cheatgrass & 4 & & 6 & 6 & & & & & & & & & 1.33 \\
\hline 2 & $2-138-1$ & Chenopodium leptophyllum & slimleaf goosefoot & 1 & 5 & 5 & 4 & & & & & & & & & 1.25 \\
\hline 2 & $2-138-1$ & Poa sandbergii & Sandberg's bluegrass & & & & 35 & & & & & & & & & 2.92 \\
\hline 2 & $2-138-1$ & Salsola kali & Russian thistle & 21 & 14 & 24 & 13 & & & & & & & & & 6.00 \\
\hline 2 & $2-138-1$ & Sisymbrium altissimum & Jim Hill's tumblemustard & 3 & 9 & 6 & 3 & & & & & & & & & 1.75 \\
\hline 2 & $2-139-1$ & Agropyron spicatum & bluebunch wheatgrass & & & & & 2 & 2 & 7 & & & & & & 0.92 \\
\hline 2 & $2-139-1$ & Amsinckia tessellata & devil's lettuce & & 2 & & & 1 & & 1 & & & & & & 0.33 \\
\hline 2 & $2-139-1$ & Artemisia tridentata & big sagebrush & 1 & & 2 & 1 & & 1 & & & & & & & 0.42 \\
\hline 2 & $2-139-1$ & Bromus tectorum & cheatgrass & 9 & & 2 & 5 & 4 & & & & & & & & 1.67 \\
\hline 2 & $2-139-1$ & Chenopodium leptophyllum & slimleaf goosefoot & 1 & 1 & & 2 & 2 & 2 & 7 & & & & & & 1.25 \\
\hline 2 & $2-139-1$ & Lactuca serriola & prickly lettuce & & 1 & & 1 & & 2 & 1 & & & & & & 0.42 \\
\hline 2 & $2-139-1$ & Salsola kali & Russian thistle & 8 & 16 & 41 & 28 & 15 & 9 & 4 & & & & & & 10.08 \\
\hline 2 & $2-139-1$ & Sisymbrium altissimum & Jim Hill's tumblemustard & & 1 & & 1 & & 2 & 1 & & & & & & 0.42 \\
\hline 2 & $2-139-1$ & Sitanion hystrix & bottlebrush grass & & 6 & 4 & & 5 & 3 & 15 & & & & & & 2.75 \\
\hline 2 & $2-50-1$ & Agropyron spicatum & bluebunch wheatgrass & & 14 & 6 & & & & 2 & 2 & 3 & 12 & 19 & 10 & 5.67 \\
\hline 2 & $2-50-1$ & Amsinckia lycopsoides & fiddleneck & 4 & 1 & & & & & 2 & & & & & & 0.58 \\
\hline 2 & $2-50-1$ & Bromus tectorum & cheatgrass & 10 & & 1 & & & & & & & & & & 0.92 \\
\hline 2 & $2-50-1$ & Calochortus macrocarpus & sagebrush mariposa lily & & 1 & & & & & & & & & & & 0.08 \\
\hline 2 & $2-50-1$ & Chrysothamnus nauseosus & gray rabbitbrush & & & & 1 & & 1 & & & & 1 & & & 0.25 \\
\hline 2 & $2-50-1$ & Crepis atribarba & slender hawksbeard & & 6 & 3 & 4 & 2 & 3 & 4 & 1 & 5 & & & 1 & 2.42 \\
\hline 2 & $2-50-1$ & Descurainia pinnata & western tansymustard & & & 2 & & & & & & & & & & 0.17 \\
\hline 2 & $2-50-1$ & Erigeron piperianus & Piper's daisy & & & & & & & & & & & & & 0.00 \\
\hline 2 & $2-50-1$ & Helianthus cusickii & Cusick's sunflower & & & & 2 & 1 & & & & & 1 & & & 0.33 \\
\hline 2 & $2-50-1$ & Lactuca serriola & prickly lettuce & 1 & & & & & & & & & 1 & & & 0.17 \\
\hline 2 & $2-50-1$ & Lupinus sericeus & silky lupine & & & & & & & & & & 1 & & & 0.08 \\
\hline 2 & $2-50-1$ & Oryzopsis hymenoides & indian ricegrass & & & & & & & 1 & & & & & & 0.08 \\
\hline 2 & $2-50-1$ & Phlox longifolia & longleaf phlox & & 2 & 1 & & & & & & & & & & 0.25 \\
\hline
\end{tabular}


Table C.2. (contd)

\begin{tabular}{|c|c|c|c|c|c|c|c|c|c|c|c|c|c|c|c|c|}
\hline $\begin{array}{c}\text { Reveg } \\
\text { Unit }\end{array}$ & Site Name & Genus/Species & Common Name & $1 \mathrm{~m}$ & $5 \mathrm{~m}$ & $\begin{array}{l}10 \\
\mathrm{~m}\end{array}$ & $\begin{array}{l}15 \\
\mathrm{~m}\end{array}$ & $\begin{array}{l}18 \\
\mathrm{~m}\end{array}$ & $\begin{array}{l}22 \\
\mathrm{~m} \\
\end{array}$ & $\begin{array}{l}26 \\
\mathrm{~m} \\
\end{array}$ & $\begin{array}{l}30 \\
\mathrm{~m}\end{array}$ & $\begin{array}{l}35 \\
\mathrm{~m}\end{array}$ & $\begin{array}{l}40 \\
\mathrm{~m} \\
\end{array}$ & $\begin{array}{l}44 \\
\mathrm{~m} \\
\end{array}$ & $\begin{array}{l}48 \\
\mathrm{~m} \\
\end{array}$ & $\begin{array}{c}\text { Mean } \\
\text { Density }\end{array}$ \\
\hline 2 & $2-50-1$ & Poa sandbergii & Sandberg's bluegrass & 19 & 20 & 55 & 25 & 33 & 26 & 22 & 46 & 27 & 36 & 34 & 12 & 29.58 \\
\hline 2 & $2-50-1$ & Salsola kali & Russian thistle & 20 & 2 & 2 & & & & 2 & & & 7 & 4 & 15 & 4.33 \\
\hline 2 & $2-50-1$ & Sisymbrium altissimum & Jim Hill's tumblemustard & 1 & & & & & & & & & & & & 0.08 \\
\hline 2 & $2-50-1$ & Stipa comata & needle-and-thread grass & & & & & & & & & & 1 & & & 0.08 \\
\hline 2 & 2-DS146-X & Achillea millefolium & yarrow & & & & & & & & & & & & 1 & 0.08 \\
\hline 2 & 2-DS146-X & Agropyron spicatum & bluebunch wheatgrass & 2 & & 4 & 5 & 5 & 2 & 23 & 3 & 12 & 5 & 3 & 2 & 5.50 \\
\hline 2 & 2-DS146-X & Amsinckia lycopsoides & fiddleneck & 1 & & 1 & 4 & & & & & 4 & 4 & & 2 & 1.33 \\
\hline 2 & 2-DS146-X & Artemisia tridentata & big sagebrush & & & & 1 & & & 4 & & 11 & 6 & 17 & 6 & 3.75 \\
\hline 2 & 2-DS146-X & Astragalus caricinus & buckwheat milkvetch & & 1 & & & & & & & & & & & 0.08 \\
\hline 2 & 2-DS146-X & Astragalus succumbens & crouching milkvetch & & 2 & 2 & & & & & & & & & & 0.33 \\
\hline 2 & 2-DS146-X & Bromus tectorum & cheatgrass & & & & & & 3 & & & & & & & 0.25 \\
\hline 2 & 2-DS146-X & Chaenactis douglasii & hoary falseyarrow & & 3 & 3 & & & & & & & & & & 0.50 \\
\hline 2 & 2-DS146-X & Chenopodium leptophyllum & slimleaf goosefoot & & & & 1 & & & & & & & & & 0.08 \\
\hline 2 & 2-DS146-X & Crepis atribarba & slender hawksbeard & 3 & & & & & & & & & & & & 0.25 \\
\hline 2 & 2-DS146-X & Lupinus sericeus & silky lupine & & & 1 & & & 4 & & & & & & & 0.42 \\
\hline 2 & 2-DS146-X & Machaeranthera canescens & hoary aster & & 17 & 4 & & & & & & 1 & & & & 1.83 \\
\hline 2 & 2-DS146-X & Poa sandbergii & Sandberg's bluegrass & 1 & & 1 & 17 & 4 & & 17 & 25 & 13 & 4 & 10 & 15 & 8.92 \\
\hline 2 & 2-DS146-X & Salsola kali & Russian thistle & 5 & 2 & 7 & 13 & 23 & 4 & 8 & 14 & 16 & 10 & 8 & 17 & 10.58 \\
\hline 2 & 2-DS146-X & Sisymbrium altissimum & Jim Hill's tumblemustard & 1 & & & 4 & 5 & & 3 & & 2 & 4 & 4 & 3 & 2.17 \\
\hline 2 & 2-DS146-X & Sitanion hystrix & bottlebrush grass & & & & 1 & & & & & & & & & 0.08 \\
\hline 2 & 2-DS60-Xa & Achillea millefolium & yarrow & & 1 & & & & & & & & & & & 0.08 \\
\hline 2 & 2-DS60-Xa & Agropyron spicatum & bluebunch wheatgrass & & & 11 & 11 & & 5 & & & & & & & 2.25 \\
\hline 2 & 2-DS60-Xa & Ambrosia acanthicarpa & bur ragweed & & & & 1 & & & & & & & & & 0.08 \\
\hline 2 & 2-DS60-Xa & Amsinckia tessellata & devil's lettuce & & & & & & & & & & & & & 0.00 \\
\hline 2 & 2-DS60-Xa & Artemisia tridentata & big sagebrush & 7 & 3 & 1 & & & & & & & & & & 0.92 \\
\hline 2 & 2-DS60-Xa & Chenopodium leptophyllum & slimleaf goosefoot & & & & 1 & 1 & & & & & & & & 0.17 \\
\hline 2 & 2-DS60-Xa & Crepis atribarba & slender hawksbeard & & & 7 & 3 & & 1 & & & & & & & 0.92 \\
\hline 2 & 2-DS60-Xa & Machaeranthera canescens & hoary aster & & 2 & 1 & 1 & & & & & & & & & 0.33 \\
\hline 2 & 2-DS60-Xa & Poa ampla Merr. & 'Sherman' big bluegrass & 2 & 1 & & & & 2 & & & & & & & 0.42 \\
\hline 2 & 2-DS60-Xa & Poa sandbergii & Sandberg's bluegrass & 10 & 22 & & 35 & 19 & 3 & & & & & & & 7.42 \\
\hline
\end{tabular}


Table C.2. (contd)

\begin{tabular}{|c|c|c|c|c|c|c|c|c|c|c|c|c|c|c|c|c|}
\hline $\begin{array}{c}\text { Reveg } \\
\text { Unit }\end{array}$ & Site Name & Genus/Species & Common Name & $1 \mathrm{~m}$ & $5 \mathrm{~m}$ & $\begin{array}{l}10 \\
\mathrm{~m}\end{array}$ & $\begin{array}{l}15 \\
\mathrm{~m}\end{array}$ & $\begin{array}{l}18 \\
\mathrm{~m}\end{array}$ & $\begin{array}{l}22 \\
\mathrm{~m}\end{array}$ & $\begin{array}{l}26 \\
\mathrm{~m}\end{array}$ & $\begin{array}{l}30 \\
\mathrm{~m}\end{array}$ & $\begin{array}{l}35 \\
\mathrm{~m}\end{array}$ & $\begin{array}{l}40 \\
\mathrm{~m}\end{array}$ & $\begin{array}{l}44 \\
\mathrm{~m}\end{array}$ & $\begin{array}{l}48 \\
\mathrm{~m}\end{array}$ & $\begin{array}{c}\text { Mean } \\
\text { Density }\end{array}$ \\
\hline 2 & 2-DS60-Xa & Salsola kali & Russian thistle & & & & 1 & & & & & & & & & 0.08 \\
\hline 2 & 2-DS60-Xa & Sisymbrium altissimum & Jim Hill's tumblemustard & 1 & 2 & 2 & 1 & 7 & 1 & & & & & & & 1.17 \\
\hline 2 & 2-DS60-Xa & Sitanion hystrix & bottlebrush grass & 41 & 49 & 10 & 3 & 21 & 18 & & & & & & & 11.83 \\
\hline 2 & 2-DS60-Xb & Agropyron spicatum & bluebunch wheatgrass & 44 & 11 & 7 & 16 & 1 & 8 & & & & & & & 7.25 \\
\hline 2 & 2-DS60-Xb & Ambrosia acanthicarpa & bur ragweed & 2 & 1 & & & & 1 & & & & & & & 0.33 \\
\hline 2 & 2-DS60-Xb & Artemisia tridentata & big sagebrush & 11 & 2 & 3 & 2 & & 1 & & & & & & & 1.58 \\
\hline 2 & 2-DS60-Xb & Astragalus succumbens & crouching milkvetch & & 1 & & & & & & & & & & & 0.08 \\
\hline 2 & 2-DS60-Xb & Balsamorhiza careyana & Carey's balsamroot & 1 & & & & & & & & & & & & 0.08 \\
\hline 2 & 2-DS60-Xb & Chenopodium leptophyllum & slimleaf goosefoot & 1 & 3 & & 1 & & 5 & & & & & & & 0.83 \\
\hline 2 & 2-DS60-Xb & Crepis atribarba & slender hawksbeard & & & & & & 1 & & & & & & & 0.08 \\
\hline 2 & 2-DS60-Xb & Festuca octoflora & slender sixweeks & & 1 & & & & & & & & & & & 0.08 \\
\hline 2 & 2-DS60-Xb & Machaeranthera canescens & hoary aster & 19 & 5 & 2 & 1 & 4 & & & & & & & & 2.58 \\
\hline 2 & 2-DS60-Xb & Poa ampla Merr. & 'Sherman' big bluegrass & 0 & 16 & 5 & 4 & 7 & & & & & & & & 2.67 \\
\hline 2 & 2-DS60-Xb & Poa sandbergii & Sandberg's bluegrass & 52 & 6 & 16 & 21 & 16 & 3 & & & & & & & 9.50 \\
\hline 2 & 2-DS60-Xb & Sisymbrium altissimum & Jim Hill's tumblemustard & & 1 & & & 1 & 2 & & & & & & & 0.33 \\
\hline 2 & 2-DS60-Xb & Sitanion hystrix & bottlebrush grass & 0 & 2 & 9 & 1 & 2 & & & & & & & & 1.17 \\
\hline 2 & 2-HQ-1 & Achillea millefolium & yarrow & & & 1 & 2 & 1 & & & & & & & 2 & 0.50 \\
\hline 2 & 2-HQ-1 & Agropyron spicatum & bluebunch wheatgrass & 1 & 1 & & 3 & 3 & & & 15 & 2 & 2 & 4 & & 2.58 \\
\hline 2 & 2-HQ-1 & Artemisia tridentata & big sagebrush & & & 1 & & & & & & & 1 & & & 0.17 \\
\hline 2 & 2-HQ-1 & Erigeron piperianus & Piper's daisy & & & & 1 & & & & & & & & & 0.08 \\
\hline 2 & 2-HQ-1 & Melilotus sp. & & & & & 3 & 3 & & & & & & & & 0.50 \\
\hline 2 & 2-HQ-1 & Phlox longifolia & longleaf phlox & & & & & & & 1 & & & & & & 0.08 \\
\hline 2 & 2-HQ-1 & Poa sandbergii & Sandberg's bluegrass & 24 & 8 & 7 & 9 & 2 & 6 & 12 & 4 & 3 & & 5 & 7 & 7.25 \\
\hline 2 & 2-HQ-1 & Salsola kali & Russian thistle & 1 & 4 & 5 & 3 & 2 & 1 & 1 & 3 & 1 & 1 & 2 & 3 & 2.25 \\
\hline 2 & 2-HQ-1 & Sitanion hystrix & bottlebrush grass & 6 & 23 & 49 & 5 & 36 & 33 & 19 & 45 & 37 & 54 & 18 & 38 & 30.25 \\
\hline 2 & 2-HQ-2 & Achillea millefolium & yarrow & & 1 & & & & & & & 1 & & & 1 & 0.25 \\
\hline 2 & 2-HQ-2 & Agropyron spicatum & bluebunch wheatgrass & 3 & 6 & 10 & 9 & 5 & 12 & 9 & 13 & 7 & 2 & 10 & 9 & 7.92 \\
\hline 2 & 2-HQ-2 & Lactuca serriola & prickly lettuce & & & & & & & & & 1 & & 1 & & 0.17 \\
\hline 2 & 2-HQ-2 & Poa sandbergii & Sandberg's bluegrass & 5 & 30 & 56 & 13 & 13 & 8 & 8 & 17 & 12 & & & 16 & 14.83 \\
\hline 2 & 2-HQ-2 & Salsola kali & Russian thistle & 3 & 2 & 2 & 5 & 4 & 1 & 4 & 6 & 2 & 3 & 3 & 1 & 3.00 \\
\hline
\end{tabular}


Table C.2. (contd)

\begin{tabular}{|c|c|c|c|c|c|c|c|c|c|c|c|c|c|c|c|c|}
\hline $\begin{array}{c}\text { Reveg } \\
\text { Unit }\end{array}$ & Site Name & Genus/Species & Common Name & $1 \mathrm{~m}$ & $5 \mathrm{~m}$ & $\begin{array}{l}10 \\
\mathrm{~m}\end{array}$ & $\begin{array}{l}15 \\
\mathrm{~m}\end{array}$ & $\begin{array}{l}18 \\
\mathrm{~m}\end{array}$ & $\begin{array}{l}22 \\
\mathrm{~m}\end{array}$ & $\begin{array}{l}26 \\
\mathrm{~m}\end{array}$ & $\begin{array}{l}30 \\
\mathrm{~m}\end{array}$ & $\begin{array}{l}35 \\
\mathrm{~m}\end{array}$ & $\begin{array}{l}40 \\
\mathrm{~m}\end{array}$ & $\begin{array}{l}44 \\
\mathrm{~m}\end{array}$ & $\begin{array}{l}48 \\
\mathrm{~m}\end{array}$ & $\begin{array}{c}\text { Mean } \\
\text { Density }\end{array}$ \\
\hline 2 & $2-\mathrm{HQ}-2$ & Sisymbrium altissimum & Jim Hill's tumblemustard & 1 & & & & & & & & & & 1 & 1 & 0.25 \\
\hline 2 & 2-HQ-2 & Sitanion hystrix & bottlebrush grass & 58 & 15 & 13 & 5 & 9 & 31 & 15 & 12 & 15 & 2 & 19 & 25 & 18.25 \\
\hline 2 & 2-HQ-2 & Vulpia myuros (L.) C.C. Gmel & rat-tail fescue & & & & & & & & 1 & & & 1 & & 0.17 \\
\hline 2 & 2-HQ-3 & Achillea millefolium & yarrow & & & 1 & & 2 & & & 2 & & & & & 0.42 \\
\hline 2 & 2-HQ-3 & Agropyron spicatum & bluebunch wheatgrass & 2 & 2 & 2 & 9 & & 14 & 13 & 9 & 10 & 3 & 8 & 8 & 6.67 \\
\hline 2 & 2-HQ-3 & Artemisia tridentata & big sagebrush & & & & & & & 1 & 1 & 2 & & & & 0.33 \\
\hline 2 & 2-HQ-3 & Balsamorhiza careyana & Carey's balsamroot & & & 1 & & 1 & & & 3 & & & & & 0.42 \\
\hline 2 & 2-HQ-3 & Bromus tectorum & cheatgrass & & & & & & & 1 & 1 & & 2 & & & 0.33 \\
\hline 2 & 2-HQ-3 & Chenopodium leptophyllum & slimleaf goosefoot & & & & & & & & & 1 & & & & 0.08 \\
\hline 2 & 2-HQ-3 & Festuca octoflora & slender sixweeks & & & 2 & & 1 & & & & & & & & 0.25 \\
\hline 2 & 2-HQ-3 & Machaeranthera canescens & hoary aster & & & & & 2 & & & 6 & & & & & 0.67 \\
\hline 2 & 2-HQ-3 & Melilotus alba & white sweetclover & & & 1 & & & & & & & & & & 0.08 \\
\hline 2 & 2-HQ-3 & Poa sandbergii & Sandberg's bluegrass & & & 13 & 8 & & 11 & 5 & & & & & & 3.08 \\
\hline 2 & 2-HQ-3 & Salsola kali & Russian thistle & & 21 & 6 & & 46 & 2 & 12 & 2 & 5 & 12 & 1 & 4 & 9.25 \\
\hline 2 & 2-HQ-3 & Sisymbrium altissimum & Jim Hill's tumblemustard & 2 & 1 & 5 & 1 & 8 & 4 & & 4 & 3 & 2 & & & 2.50 \\
\hline 2 & 2-HQ-3 & Sitanion hystrix & bottlebrush grass & 68 & 51 & 54 & 28 & 29 & 36 & 23 & 79 & 49 & 51 & 53 & 65 & 48.83 \\
\hline 2 & 2-HQ-3 & Vulpia myuros (L.) C.C. Gmel & rat-tail fescue & & 3 & & & & & & 8 & 3 & & & & 1.17 \\
\hline 2 & 2-HW-1 & Agropyron spicatum & bluebunch wheatgrass & 7 & 3 & & 5 & 13 & 14 & 5 & 6 & 4 & 7 & 10 & 7 & 6.75 \\
\hline 2 & 2-HW-1 & Amsinckia lycopsoides & fiddleneck & 1 & 1 & 2 & & 4 & 2 & 4 & 2 & 1 & 4 & 2 & & 1.92 \\
\hline 2 & 2-HW-1 & Artemisia tridentata & big sagebrush & & & & & & 1 & & & & & & 1 & 0.17 \\
\hline 2 & 2-HW-1 & Balsamorhiza careyana & Carey's balsamroot & & & & & & & & & & 1 & & & 0.08 \\
\hline 2 & 2-HW-1 & Bromus tectorum & cheatgrass & 1 & & & & & & & & & & & 1 & 0.17 \\
\hline 2 & 2-HW-1 & Chenopodium leptophyllum & slimleaf goosefoot & 1 & 1 & 1 & 5 & 2 & & & 2 & 1 & & 3 & 2 & 1.50 \\
\hline 2 & 2-HW-1 & Crepis atribarba & slender hawksbeard & & & & & & & & & & & & 2 & 0.17 \\
\hline 2 & 2-HW-1 & Descurainia pinnata & western tansymustard & & & & 1 & & & & & & & & & 0.08 \\
\hline 2 & 2-HW-1 & Erodium cicutarium & storksbill & & & & & 1 & & & & & & & & 0.08 \\
\hline 2 & 2-HW-1 & Lactuca serriola & prickly lettuce & & 1 & & & & & 1 & & 1 & & & & 0.25 \\
\hline 2 & 2-HW-1 & Poa sandbergii & Sandberg's bluegrass & 3 & 1 & & 4 & 11 & 24 & 5 & 1 & 4 & 17 & 9 & 5 & 7.00 \\
\hline 2 & 2-HW-1 & Salsola kali & Russian thistle & & & 1 & & 1 & & & & & 1 & & & 0.25 \\
\hline 2 & 2-HW-1 & Sisymbrium altissimum & Jim Hill's tumblemustard & 2 & 37 & 13 & 17 & 8 & 10 & 18 & 17 & 19 & 8 & 5 & 13 & 13.92 \\
\hline
\end{tabular}


Table C.2. (contd)

\begin{tabular}{|c|c|c|c|c|c|c|c|c|c|c|c|c|c|c|c|c|}
\hline $\begin{array}{c}\text { Reveg } \\
\text { Unit }\end{array}$ & Site Name & Genus/Species & Common Name & $1 \mathrm{~m}$ & $5 \mathrm{~m}$ & $\begin{array}{l}10 \\
\mathrm{~m}\end{array}$ & $\begin{array}{l}15 \\
\mathrm{~m}\end{array}$ & $\begin{array}{l}18 \\
\mathrm{~m}\end{array}$ & $\begin{array}{l}22 \\
\mathrm{~m}\end{array}$ & $\begin{array}{l}26 \\
\mathrm{~m}\end{array}$ & $\begin{array}{l}30 \\
\mathrm{~m}\end{array}$ & $\begin{array}{l}35 \\
\mathrm{~m}\end{array}$ & $\begin{array}{l}40 \\
\mathrm{~m}\end{array}$ & $\begin{array}{l}44 \\
\mathrm{~m}\end{array}$ & $\begin{array}{l}48 \\
\mathrm{~m}\end{array}$ & $\begin{array}{c}\text { Mean } \\
\text { Density }\end{array}$ \\
\hline 2 & 2-HW-1 & Sitanion hystrix & bottlebrush grass & 48 & 12 & 21 & 13 & 19 & 78 & 26 & 8 & 49 & 26 & 29 & 48 & 31.42 \\
\hline 2 & 2-HW-2 & Agropyron spicatum & bluebunch wheatgrass & 1 & 5 & 9 & & 10 & 5 & 9 & 2 & 16 & 10 & 8 & & 6.25 \\
\hline 2 & 2-HW-2 & Amsinckia lycopsoides & fiddleneck & 3 & & & & & & & 5 & 1 & & & 5 & 1.17 \\
\hline 2 & 2-HW-2 & Artemisia tridentata & big sagebrush & & & & & & & & 1 & & & & & 0.08 \\
\hline 2 & 2-HW-2 & Bromus tectorum & cheatgrass & & & & & & 51 & 5 & & & & & & 4.67 \\
\hline 2 & 2-HW-2 & Chenopodium leptophyllum & slimleaf goosefoot & & & 1 & & & & & & 2 & & & 1 & 0.33 \\
\hline 2 & 2-HW-2 & Descurainia pinnata & western tansymustard & & & & & & & & & & & & & 0.00 \\
\hline 2 & 2-HW-2 & Elymus cinereus & giant wildrye & & & & & & & 1 & & & & & & 0.08 \\
\hline 2 & 2-HW-2 & Epilobium paniculatum & tall willowherb & & & & & & & 3 & & & & & & 0.25 \\
\hline 2 & 2-HW-2 & Lactuca serriola & prickly lettuce & 1 & 1 & 2 & 3 & & 4 & 1 & & 3 & 1 & & 2 & 1.50 \\
\hline 2 & 2-HW-2 & Lupinus sericeus & silky lupine & & 65 & & & & & & & & & & & 5.42 \\
\hline 2 & 2-HW-2 & Machaeranthera canescens & hoary aster & 1 & & & & & & & 1 & & & & 5 & 0.58 \\
\hline 2 & 2-HW-2 & Poa sandbergii & Sandberg's bluegrass & & & 7 & & 32 & & & 3 & 2 & & 4 & & 4.00 \\
\hline 2 & 2-HW-2 & Salsola kali & Russian thistle & & & & & & & & & & & & & 0.00 \\
\hline 2 & 2-HW-2 & Sisymbrium altissimum & Jim Hill's tumblemustard & 2 & & 3 & 3 & 51 & 6 & 6 & 20 & & 5 & 2 & 9 & 8.92 \\
\hline 2 & 2-HW-2 & Sitanion hystrix & bottlebrush grass & 71 & 45 & 68 & & & 22 & 91 & 50 & 146 & 103 & 73 & 46 & 59.58 \\
\hline 3 & 3-RT-1 & Agastache occidentalis & western horsemint & & & & & & & 1 & & & & & & 0.08 \\
\hline 3 & 3-RT-1 & Agropyron spicatum & bluebunch wheatgrass & 30 & 8 & 18 & 34 & 7 & 6 & 15 & 19 & 33 & 50 & 35 & 12 & 22.25 \\
\hline 3 & 3-RT-1 & Bromus tectorum & cheatgrass & & & & & 14 & 7 & 6 & 3 & 11 & 2 & 1 & 7 & 4.25 \\
\hline 3 & 3-RT-1 & Chenopodium leptophyllum & slimleaf goosefoot & & & & & & 3 & & & & & & & 0.25 \\
\hline 3 & 3-RT-1 & Crepis atribarba & slender hawksbeard & & & & & 1 & & & & & & & & 0.08 \\
\hline 3 & $3-\mathrm{RT}-1$ & Festuca idahoensis & Idaho fescue & 14 & & & & & & & & & & & & 1.17 \\
\hline 3 & 3-RT-1 & Lomatium grayi & Gray’s desertparsley & & & & & & 1 & & & & & & & 0.08 \\
\hline 3 & 3-RT-1 & Phacelia hastata & whiteleaf scorpionweed & & & 1 & & & & & 3 & & & 3 & 1 & 0.67 \\
\hline 3 & 3-RT-1 & Poa sandbergii & Sandberg's bluegrass & 85 & 16 & 50 & 21 & 31 & 12 & 14 & 9 & 6 & 11 & 15 & 8 & 23.17 \\
\hline 3 & 3-RT-1 & Salsola kali & Russian thistle & 1 & & & & & & & & & & & & 0.08 \\
\hline 3 & 3-RT-1 & Sisymbrium altissimum & Jim Hill's tumblemustard & & & & & 2 & 1 & & & & 1 & & 2 & 0.50 \\
\hline 3 & 3-RT-2 & Agropyron spicatum & bluebunch wheatgrass & 22 & 31 & 12 & 25 & 31 & 26 & 52 & 13 & 10 & 4 & 5 & 1 & 19.33 \\
\hline 3 & 3-RT-2 & Bromus tectorum & cheatgrass & 8 & 2 & & 2 & 2 & & 1 & 21 & 1 & & & & 3.08 \\
\hline 3 & 3-RT-2 & Chenopodium leptophyllum & slimleaf goosefoot & 1 & 1 & & 2 & 2 & & & & & & & & 0.50 \\
\hline
\end{tabular}


Table C.2. (contd)

\begin{tabular}{|c|c|c|c|c|c|c|c|c|c|c|c|c|c|c|c|c|}
\hline $\begin{array}{c}\text { Reveg } \\
\text { Unit }\end{array}$ & Site Name & Genus/Species & Common Name & $1 \mathrm{~m}$ & $5 \mathrm{~m}$ & $\begin{array}{l}10 \\
\mathrm{~m}\end{array}$ & $\begin{array}{l}15 \\
\mathrm{~m}\end{array}$ & $\begin{array}{l}18 \\
\mathrm{~m}\end{array}$ & $\begin{array}{l}22 \\
\mathrm{~m}\end{array}$ & $\begin{array}{l}26 \\
\mathrm{~m}\end{array}$ & $\begin{array}{l}30 \\
\mathrm{~m}\end{array}$ & $\begin{array}{l}35 \\
\mathrm{~m}\end{array}$ & $\begin{array}{l}40 \\
\mathrm{~m}\end{array}$ & $\begin{array}{l}44 \\
\mathrm{~m}\end{array}$ & $\begin{array}{l}48 \\
\mathrm{~m}\end{array}$ & $\begin{array}{c}\text { Mean } \\
\text { Density }\end{array}$ \\
\hline 3 & 3-RT-2 & Festuca idahoensis & Idaho fescue & & 2 & & 2 & 2 & 2 & 2 & 3 & & & & & 1.08 \\
\hline 3 & 3-RT-2 & Lupinus sericeus & silky lupine & & & & & & & 1 & & & & & & 0.08 \\
\hline 3 & 3-RT-2 & Phacelia hastata & whiteleaf scorpionweed & & 1 & & & 3 & 1 & 3 & 1 & & & & & 0.75 \\
\hline 3 & $3-\mathrm{RT}-2$ & Poa sandbergii & Sandberg's bluegrass & 102 & 92 & 87 & 83 & 106 & 72 & 55 & 40 & 15 & 24 & 15 & 6 & 58.08 \\
\hline 3 & $3-\mathrm{RT}-2$ & Salsola kali & Russian thistle & & & & & & & & 2 & 22 & 1 & 1 & & 2.17 \\
\hline 3 & $3-\mathrm{RT}-2$ & Sisymbrium altissimum & Jim Hill's tumblemustard & & 1 & & 1 & & & & 1 & & & & & 0.25 \\
\hline 3 & $3-\mathrm{RT}-3$ & Agropyron spicatum & bluebunch wheatgrass & 22 & 9 & 22 & 19 & 22 & 31 & 17 & 17 & 13 & 20 & 24 & 23 & 19.92 \\
\hline 3 & $3-\mathrm{RT}-3$ & Bromus japonicus & Japanese brome & & & & & & & & 1 & & & & & 0.08 \\
\hline 3 & $3-\mathrm{RT}-3$ & Bromus tectorum & cheatgrass & 1 & 2 & 1 & & & 1 & 2 & & 1 & & & & 0.67 \\
\hline 3 & 3-RT-3 & Crepis modocensis & low hawksbeard & & & & & 1 & & & & & & & & 0.08 \\
\hline 3 & $3-\mathrm{RT}-3$ & Phacelia hastata & whiteleaf scorpionweed & & & & & & & & & 1 & & & 1 & 0.17 \\
\hline 3 & $3-\mathrm{RT}-3$ & Poa sandbergii & Sandberg's bluegrass & 31 & 6 & 8 & 6 & 10 & 5 & 12 & 16 & 4 & 15 & 16 & 17 & 12.17 \\
\hline 3 & $3-\mathrm{RT}-3$ & Salsola kali & Russian thistle & & & & & & 1 & 1 & & 2 & & 1 & 2 & 0.58 \\
\hline 4 & $4-623 \mathrm{~A}-1$ & Agropyron spicatum & bluebunch wheatgrass & & & 7 & & & & & & & & & & 0.58 \\
\hline 4 & $4-623 \mathrm{~A}-1$ & Bromus tectorum & cheatgrass & & & & & & 2 & & 1 & & & & & 0.25 \\
\hline 4 & $4-623 \mathrm{~A}-1$ & Phacelia hastata & whiteleaf scorpionweed & & & 1 & & & & & & & & & & 0.08 \\
\hline 4 & $4-623 \mathrm{~A}-1$ & Poa sandbergii & Sandberg's bluegrass & 1 & & & 2 & 3 & & 2 & 3 & & 8 & 3 & 1 & 1.92 \\
\hline 4 & $4-623 \mathrm{~A}-1$ & Polygonum aviculare & doorweed & & & & & & & 1 & & & & & & 0.08 \\
\hline 4 & $4-623 \mathrm{~A}-1$ & Salsola kali & Russian thistle & & 3 & & 1 & & 3 & & & & & & 1 & 0.67 \\
\hline 4 & $4-623 \mathrm{~A}-1$ & Sisymbrium altissimum & Jim Hill's tumblemustard & & & & & & 1 & & & & & & & 0.08 \\
\hline 4 & 4-623A-road & Achillea millefolium & yarrow & & & & 2 & & & & & & & & & 0.17 \\
\hline 4 & 4-623A-road & Agropyron spicatum & bluebunch wheatgrass & 2 & 2 & & 1 & 1 & & & & & & & & 0.50 \\
\hline 4 & 4-623A-road & Balsamorhiza careyana & Carey's balsamroot & & & & & 1 & & & & & & & & 0.08 \\
\hline 4 & 4-623A-road & Balsamorhiza rosea & rosy balsamroot & & & & 1 & & & & & & & & & 0.08 \\
\hline 4 & 4-623A-road & Bromus tectorum & cheatgrass & 1 & & 7 & 9 & 6 & & & & & & & & 1.92 \\
\hline 4 & 4-623A-road & Lupinus sericeus & silky lupine & & 1 & & & 2 & 1 & & & & & & & 0.33 \\
\hline 4 & 4-623A-road & Phacelia hastata & whiteleaf scorpionweed & 1 & & & & & & & & & & & & 0.08 \\
\hline 4 & 4-623A-road & Poa sandbergii & Sandberg's bluegrass & 5 & 4 & 11 & 8 & 10 & 7 & & & & & & & 3.75 \\
\hline 4 & 4-623A-road & Salsola kali & Russian thistle & & 7 & & 2 & 21 & 2 & & & & & & & 2.67 \\
\hline 4 & 4-623A-road & Sisymbrium altissimum & Jim Hill's tumblemustard & & & 1 & 3 & & 1 & & & & & & & 0.42 \\
\hline
\end{tabular}


Table C.2. (contd)

\begin{tabular}{|c|c|c|c|c|c|c|c|c|c|c|c|c|c|c|c|c|}
\hline $\begin{array}{c}\text { Reveg } \\
\text { Unit }\end{array}$ & Site Name & Genus/Species & Common Name & $1 \mathrm{~m}$ & $5 \mathrm{~m}$ & $\begin{array}{l}10 \\
\mathrm{~m}\end{array}$ & $\begin{array}{l}15 \\
\mathrm{~m}\end{array}$ & $\begin{array}{l}18 \\
\mathrm{~m}\end{array}$ & $\begin{array}{l}22 \\
\mathrm{~m}\end{array}$ & $\begin{array}{l}26 \\
\mathrm{~m}\end{array}$ & $\begin{array}{l}30 \\
\mathrm{~m}\end{array}$ & $\begin{array}{l}35 \\
\mathrm{~m}\end{array}$ & $\begin{array}{l}40 \\
\mathrm{~m}\end{array}$ & $\begin{array}{l}44 \\
\mathrm{~m}\end{array}$ & $\begin{array}{l}48 \\
\mathrm{~m}\end{array}$ & $\begin{array}{c}\text { Mean } \\
\text { Density }\end{array}$ \\
\hline 4 & 4-623A-road & Sitanion hystrix & bottlebrush grass & 11 & & & 3 & & & & & & & & & 1.17 \\
\hline 4 & $4-\mathrm{C}-1$ & Agropyron spicatum & bluebunch wheatgrass & 29 & 3 & & & 1 & 11 & 6 & & 28 & 7 & 25 & 25 & 11.25 \\
\hline 4 & $4-\mathrm{C}-1$ & Festuca idahoensis & Idaho fescue & 2 & 1 & & & & & & & 2 & & 7 & 4 & 1.33 \\
\hline 4 & $4-\mathrm{C}-1$ & Phacelia hastata & whiteleaf scorpionweed & 4 & 3 & & & 1 & & & & & 1 & 9 & 24 & 3.50 \\
\hline 4 & $4-\mathrm{C}-1$ & Poa sandbergii & Sandberg's bluegrass & 74 & 37 & 25 & 91 & 473 & 71 & 41 & 120 & 22 & 5 & 165 & 73 & 99.75 \\
\hline 4 & $4-\mathrm{C}-1$ & Salsola kali & Russian thistle & 4 & 13 & & & & & & 2 & 1 & 1 & & & 1.75 \\
\hline 4 & $4-\mathrm{C}-3$ & Agastache occidentalis & western horsemint & & & & & & 1 & & & & & 1 & 2 & 0.33 \\
\hline 4 & $4-\mathrm{C}-3$ & Agropyron spicatum & bluebunch wheatgrass & 46 & 30 & 28 & 38 & 53 & 40 & 56 & 29 & 54 & 57 & 33 & 41 & 42.08 \\
\hline 4 & $4-\mathrm{C}-3$ & Bromus tectorum & cheatgrass & & & & 1 & & 1 & & & & & 1 & & 0.25 \\
\hline 4 & $4-\mathrm{C}-3$ & Erigeron linearis & desert yellowdaisy & & & & & & & & 1 & & & & & 0.08 \\
\hline 4 & $4-\mathrm{C}-3$ & Eriogonum sphaerocephalum & rock buckwheat & & & & & & & & & & & 1 & & 0.08 \\
\hline 4 & $4-\mathrm{C}-3$ & Festuca idahoensis & Idaho fescue & 8 & 2 & 5 & 5 & 13 & 1 & 7 & & 3 & 6 & & & 4.17 \\
\hline 4 & $4-\mathrm{C}-3$ & Lactuca serriola & prickly lettuce & & & & & & & & & & 1 & & & 0.08 \\
\hline 4 & $4-\mathrm{C}-3$ & Phacelia hastata & whiteleaf scorpionweed & 1 & 4 & 3 & 2 & & 10 & 7 & 2 & 1 & 4 & 3 & 1 & 3.17 \\
\hline 4 & $4-\mathrm{C}-3$ & Poa sandbergii & Sandberg's bluegrass & 77 & 20 & 54 & 128 & 142 & 55 & 144 & 70 & 109 & 79 & 109 & 58 & 87.08 \\
\hline 4 & $4-\mathrm{C}-3$ & Salsola kali & Russian thistle & 2 & & & 1 & & 4 & & 2 & & 2 & & 2 & 1.08 \\
\hline 4 & $4-C-3$ & Salvia dorrii & grayball sage & & & & 1 & & & & & & & & & 0.08 \\
\hline 4 & $4-\mathrm{C}-3$ & Sisymbrium altissimum & Jim Hill's tumblemustard & & 1 & 1 & & & & & & & & & & 0.17 \\
\hline 4 & 4-CCF-1 & Achillea millefolium & yarrow & & & & & & & & 10 & & & 1 & & 0.92 \\
\hline 4 & 4-CCF-1 & Agropyron spicatum & bluebunch wheatgrass & 6 & 6 & 14 & 10 & 11 & & & 16 & 29 & & 3 & 18 & 9.42 \\
\hline 4 & 4-CCF-1 & Astragalus purshii & woolly-pod milkvetch & 2 & & & & & & & & & & & & 0.17 \\
\hline 4 & 4-CCF-1 & Balsamorhiza rosea & rosy balsamroot & & & 2 & 1 & 2 & 8 & 4 & 3 & & 3 & 3 & & 2.17 \\
\hline 4 & 4-CCF-1 & Bromus japonicus & Japanese brome & & & & & & & & 1 & & & & & 0.08 \\
\hline 4 & 4-CCF-1 & Bromus tectorum & cheatgrass & 2 & 3 & 3 & 6 & 1 & 7 & 3 & 4 & 10 & 18 & 17 & 4 & 6.50 \\
\hline 4 & 4-CCF-1 & Chenopodium leptophyllum & slimleaf goosefoot & & 1 & & & & & & & 4 & & & 6 & 0.92 \\
\hline 4 & 4-CCF-1 & Crepis modocensis & low hawksbeard & & & & 1 & 2 & 4 & 5 & & & 2 & 5 & & 1.58 \\
\hline 4 & 4-CCF-1 & Lomatium grayi & Gray's desertparsley & & & & 1 & & & & & & & & & 0.08 \\
\hline 4 & 4-CCF-1 & Phacelia hastata & whiteleaf scorpionweed & 2 & 2 & 2 & & 1 & & 1 & & & & 1 & 1 & 0.83 \\
\hline 4 & 4-CCF-1 & Phlox hoodii & Hood's phlox & & & & & & 2 & 3 & & & & 2 & & 0.58 \\
\hline 4 & 4-CCF-1 & Poa sandbergii & Sandberg's bluegrass & 26 & 25 & 61 & 53 & 54 & 33 & 23 & 21 & 17 & 6 & 17 & 52 & 32.33 \\
\hline
\end{tabular}


Table C.2. (contd)

\begin{tabular}{|c|c|c|c|c|c|c|c|c|c|c|c|c|c|c|c|c|}
\hline $\begin{array}{c}\text { Reveg } \\
\text { Unit }\end{array}$ & Site Name & Genus/Species & Common Name & $1 \mathrm{~m}$ & $5 \mathrm{~m}$ & $\begin{array}{l}10 \\
\mathrm{~m}\end{array}$ & $\begin{array}{l}15 \\
\mathrm{~m}\end{array}$ & $\begin{array}{l}18 \\
\mathrm{~m}\end{array}$ & $\begin{array}{l}22 \\
\mathrm{~m}\end{array}$ & $\begin{array}{l}26 \\
\mathrm{~m}\end{array}$ & $\begin{array}{l}30 \\
\mathrm{~m}\end{array}$ & $\begin{array}{l}35 \\
\mathrm{~m}\end{array}$ & $\begin{array}{l}40 \\
\mathrm{~m}\end{array}$ & $\begin{array}{l}44 \\
\mathrm{~m}\end{array}$ & $\begin{array}{l}48 \\
\mathrm{~m}\end{array}$ & $\begin{array}{l}\text { Mean } \\
\text { Density }\end{array}$ \\
\hline 4 & 4-CCF-1 & Polygonum aviculare & doorweed & 3 & 2 & 4 & & & & & & & & & 1 & 0.83 \\
\hline 4 & 4-CCF-1 & Sisymbrium altissimum & Jim Hill's tumblemustard & & 1 & 1 & & & & & 1 & & 1 & 2 & & 0.50 \\
\hline 4 & 4-CCF-Berm & Agropyron spicatum & bluebunch wheatgrass & & & & 1 & & & & & & & & & 0.08 \\
\hline 4 & 4-CCF-Berm & Bromus tectorum & cheatgrass & & 6 & 1 & 22 & 12 & 5 & & & & & & & 3.83 \\
\hline 4 & 4-CCF-Berm & Phacelia hastata & whiteleaf scorpionweed & & 2 & & 14 & 5 & & & & & & & & 1.75 \\
\hline 4 & 4-CCF-Berm & Sisymbrium altissimum & Jim Hill's tumblemustard & & & & 2 & & & & & & & & & 0.17 \\
\hline 4 & 4-CCF-Road & Achillea millefolium & yarrow & 4 & & & & & & & & & & & & 0.33 \\
\hline 4 & 4-CCF-Road & Balsamorhiza rosea & rosy balsamroot & & 7 & 3 & 14 & & & & & & & & & 2.00 \\
\hline 4 & 4-CCF-Road & Bromus japonicus & Japanese brome & 2 & & & & & & & & & & & & 0.17 \\
\hline 4 & 4-CCF-Road & Bromus tectorum & cheatgrass & 23 & 4 & 14 & 10 & 32 & 3 & & & & & & & 7.17 \\
\hline 4 & 4-CCF-Road & Crepis modocensis & low hawksbeard & & 1 & 3 & & & & & & & & & & 0.33 \\
\hline 4 & 4-CCF-Road & Lomatium grayi & Gray's desertparsley & 3 & & & 2 & 1 & & & & & & & & 0.50 \\
\hline 4 & 4-CCF-Road & $\begin{array}{l}\text { Lygodesmia juncea (Pursh) D. } \\
\text { Don ex Hook. }\end{array}$ & rush skeletonplant & 4 & & 1 & & & & & & & & & & 0.42 \\
\hline 4 & 4-CCF-Road & Phacelia hastata & whiteleaf scorpionweed & & & & 4 & & 4 & & & & & & & 0.67 \\
\hline 4 & 4-CCF-Road & Poa sandbergii & Sandberg's bluegrass & 14 & 9 & 7 & & & & & & & & & & 2.50 \\
\hline 4 & 4-CCF-Road & Salsola kali & Russian thistle & & & & & & & & & & & & & 0.00 \\
\hline 4 & 4-CCF-Road & Sisymbrium altissimum & Jim Hill's tumblemustard & & & & 1 & & & & & & & & & 0.08 \\
\hline 5 & 5 -OB-1 & Agropyron spicatum & bluebunch wheatgrass & 3 & 1 & 4 & 4 & & 3 & & 5 & & 3 & 3 & 6 & 2.67 \\
\hline 5 & $5-\mathrm{OB}-1$ & Bromus tectorum & cheatgrass & & & & & & 1 & & & & & & & 0.08 \\
\hline 5 & 5-OB-1 & Chenopodium leptophyllum & slimleaf goosefoot & & & & & & & & & & 1 & & & 0.08 \\
\hline 5 & $5-\mathrm{OB}-1$ & Phacelia hastata & whiteleaf scorpionweed & 1 & & 1 & & & & 2 & 1 & & & 2 & 2 & 0.75 \\
\hline 5 & 5-OB-1 & Poa sandbergii & Sandberg's bluegrass & 11 & 2 & 8 & & 1 & 7 & & 1 & 1 & 2 & 3 & 29 & 5.42 \\
\hline 5 & $5-\mathrm{OB}-2$ & Agropyron spicatum & bluebunch wheatgrass & 10 & 1 & & & & & 1 & & & & 1 & 18 & 2.58 \\
\hline 5 & $5-\mathrm{OB}-2$ & Phacelia hastata & whiteleaf scorpionweed & 1 & & & & & & & & & & & & 0.08 \\
\hline 5 & $5-\mathrm{OB}-2$ & Poa sandbergii & Sandberg's bluegrass & 44 & 5 & 7 & 35 & 45 & 3 & & 69 & 53 & 54 & 75 & 23 & 34.42 \\
\hline 5 & $5-\mathrm{OB}-2$ & Salsola kali & Russian thistle & 2 & & & & & & 1 & & & 1 & & & 0.33 \\
\hline 6 & $6-6635-X$ & Agastache occidentalis & western horsemint & & & & 1 & & & & & & & & & 0.08 \\
\hline 6 & $6-6635-X$ & Agropyron spicatum & bluebunch wheatgrass & 27 & 46 & 29 & 17 & 31 & 6 & 9 & 26 & 36 & 5 & 49 & 23 & 25.33 \\
\hline 6 & 6-6635-X & Astragalus purshii & woolly-pod milkvetch & & 1 & & & & & & & & & & & 0.08 \\
\hline 6 & $6-6635-X$ & Bromus tectorum & cheatgrass & 1 & & & 1 & & & 2 & 1 & 3 & & & & 0.67 \\
\hline
\end{tabular}


Table C.2. (contd)

\begin{tabular}{|c|c|c|c|c|c|c|c|c|c|c|c|c|c|c|c|c|}
\hline $\begin{array}{c}\text { Reveg } \\
\text { Unit }\end{array}$ & Site Name & Genus/Species & Common Name & $1 \mathrm{~m}$ & $5 \mathrm{~m}$ & $\begin{array}{l}10 \\
\mathrm{~m}\end{array}$ & $\begin{array}{l}15 \\
\mathrm{~m}\end{array}$ & $\begin{array}{l}18 \\
\mathrm{~m}\end{array}$ & $\begin{array}{l}22 \\
\mathrm{~m}\end{array}$ & $\begin{array}{l}26 \\
\mathrm{~m}\end{array}$ & $\begin{array}{l}30 \\
\mathrm{~m}\end{array}$ & $\begin{array}{l}35 \\
\mathrm{~m}\end{array}$ & $\begin{array}{l}40 \\
\mathrm{~m}\end{array}$ & $\begin{array}{l}44 \\
\mathrm{~m}\end{array}$ & $\begin{array}{l}48 \\
\mathrm{~m}\end{array}$ & $\begin{array}{c}\text { Mean } \\
\text { Density }\end{array}$ \\
\hline 6 & $6-6635-X$ & Chenopodium leptophyllum & slimleaf goosefoot & & 1 & 3 & & & & 4 & 1 & 2 & 1 & & & 1.00 \\
\hline 6 & $6-6635-X$ & Descurainia pinnata & western tansymustard & & & & & & & & & & 1 & & & 0.08 \\
\hline 6 & $6-6635-X$ & Descurainia sophia & flixweed & & & & & & & & & & & & & 0.00 \\
\hline 6 & $6-6635-X$ & Festuca idahoensis & Idaho fescue & & & & & & & & & & 3 & & & 0.25 \\
\hline 6 & $6-6635-X$ & Lomatium grayi & Gray's desertparsley & & & & & 1 & & & & & & & & 0.08 \\
\hline 6 & $6-6635-X$ & Phacelia hastata & whiteleaf scorpionweed & 9 & & 2 & 3 & 2 & & 12 & 44 & 30 & 1 & & 3 & 8.83 \\
\hline 6 & $6-6635-X$ & Poa sandbergii & Sandberg's bluegrass & & & & & & & & & & 15 & & & 1.25 \\
\hline 6 & $6-6635-X$ & Polygonum aviculare & doorweed & 17 & 25 & 37 & 22 & 43 & 44 & 20 & 10 & 20 & 8 & 8 & 6 & 21.67 \\
\hline 6 & $6-6635-X$ & Salsola kali & Russian thistle & & & & 1 & & & & & & & & & 0.08 \\
\hline 6 & $6-6635-X$ & Sisymbrium altissimum & Jim Hill's tumblemustard & 6 & 1 & 2 & 19 & 26 & 3 & 11 & 10 & 25 & 6 & & 3 & 9.33 \\
\hline 6 & $6-6636-1$ & Achillea millefolium & yarrow & & & & & 1 & & & & & & & & 0.08 \\
\hline 6 & $6-6636-1$ & Agropyron spicatum & bluebunch wheatgrass & 2 & 11 & 11 & 17 & 6 & 3 & 5 & 52 & 45 & 16 & 5 & 21 & 16.17 \\
\hline 6 & $6-6636-1$ & Astragalus purshii & woolly-pod milkvetch & & & & & & & & & 2 & & 1 & & 0.25 \\
\hline 6 & 6-6636-1 & Balsamorhiza rosea & rosy balsamroot & 3 & 6 & 1 & & & & & & & & & & 0.83 \\
\hline 6 & $6-6636-1$ & Bromus tectorum & cheatgrass & 1 & 3 & & & & 10 & 2 & & & & & 1 & 1.42 \\
\hline 6 & $6-6636-1$ & Crepis modocensis & low hawksbeard & 5 & 13 & 1 & 28 & 1 & 2 & & & & 1 & 10 & 1 & 5.17 \\
\hline 6 & $6-6636-1$ & Descurainia pinnata & western tansymustard & & & & & & 1 & & & & & & & 0.08 \\
\hline 6 & $6-6636-1$ & Erigeron linearis & desert yellowdaisy & & & & & & & & & 1 & & & & 0.08 \\
\hline 6 & $6-6636-1$ & Eriogonum sphaerocephalum & rock buckwheat & & & & & & & & & & & 1 & & 0.08 \\
\hline 6 & $6-6636-1$ & Haplopappus stenophylla & narrowleaf goldenweed & & & & & & & & & & 1 & & & 0.08 \\
\hline 6 & $6-6636-1$ & Lomatium sp. & & & & 2 & & & 5 & & & & & & & 0.58 \\
\hline 6 & $6-6636-1$ & Phacelia hastata & whiteleaf scorpionweed & 4 & 2 & 3 & 4 & 4 & 7 & 2 & & 1 & 1 & & 4 & 2.67 \\
\hline 6 & $6-6636-1$ & Phlox hoodii & Hood's phlox & & 5 & & & & & & & & & 3 & 5 & 1.08 \\
\hline 6 & $6-6636-1$ & Poa sandbergii & Sandberg's bluegrass & 16 & 2 & & 5 & & & & & & & 16 & 3 & 3.50 \\
\hline 6 & 6-6636-1 & Polygonum aviculare & doorweed & 8 & 8 & 8 & 3 & 1 & 1 & 3 & 2 & 5 & 6 & & 7 & 4.33 \\
\hline 6 & $6-6636-1$ & Ribes aureum & golden currant & & & & & & 1 & & & & & & & 0.08 \\
\hline 6 & $6-6636-1$ & Sisymbrium altissimum & Jim Hill's tumblemustard & 2 & & & & 3 & & & & & & & & 0.42 \\
\hline 6 & $6-6636-1$ & Sitanion hystrix & bottlebrush grass & 1 & & & & & 1 & 1 & 4 & 1 & & & 1 & 0.75 \\
\hline 7 & 7-PH-X & Achillea millefolium & yarrow & 9 & 2 & 0 & 1 & 2 & 6 & & & 2 & 1 & 1 & 6 & 2.50 \\
\hline 7 & 7-PH-X & Agastache occidentalis & western horsemint & & & & 2 & & & & & & & & & 0.17 \\
\hline
\end{tabular}


Table C.2. (contd)

\begin{tabular}{|c|c|c|c|c|c|c|c|c|c|c|c|c|c|c|c|c|}
\hline $\begin{array}{c}\text { Reveg } \\
\text { Unit }\end{array}$ & Site Name & Genus/Species & Common Name & $1 \mathrm{~m}$ & $5 \mathrm{~m}$ & $\begin{array}{l}10 \\
\mathrm{~m}\end{array}$ & $\begin{array}{l}15 \\
\mathrm{~m}\end{array}$ & $\begin{array}{l}18 \\
\mathrm{~m}\end{array}$ & $\begin{array}{l}22 \\
\mathrm{~m}\end{array}$ & $\begin{array}{l}26 \\
\mathrm{~m}\end{array}$ & $\begin{array}{l}30 \\
\mathrm{~m}\end{array}$ & $\begin{array}{l}35 \\
\mathrm{~m}\end{array}$ & $\begin{array}{l}40 \\
\mathrm{~m}\end{array}$ & $\begin{array}{l}44 \\
\mathrm{~m}\end{array}$ & $\begin{array}{l}48 \\
\mathrm{~m}\end{array}$ & $\begin{array}{c}\text { Mean } \\
\text { Density }\end{array}$ \\
\hline 7 & 7-PH-X & Agropyron spicatum & bluebunch wheatgrass & 5 & 5 & 6 & 4 & & & 3 & 3 & 2 & 5 & & & 2.75 \\
\hline 7 & 7-PH-X & Artemisia tridentata & big sagebrush & & & & & & & & & 1 & 1 & & 1 & 0.25 \\
\hline 7 & 7-PH-X & Bromus tectorum & cheatgrass & 27 & 17 & 70 & 35 & 65 & 75 & 2 & 2 & 5 & 25 & 55 & 65 & 36.92 \\
\hline 7 & 7-PH-X & Chrysothamnus nauseosus & gray rabbitbrush & & & & & & 1 & & & & & & & 0.08 \\
\hline 7 & 7-PH-X & Descurainia pinnata & western tansymustard & & & & & & & & & & & 1 & 1 & 0.17 \\
\hline 7 & 7-PH-X & Elymus cinereus & giant wildrye & & & 1 & & & & 1 & & & & & & 0.17 \\
\hline 7 & 7-PH-X & Lomatium sp. & & 2 & & 1 & 1 & 4 & & & & & 1 & & 1 & 0.83 \\
\hline 7 & 7-PH-X & Phacelia hastata & whiteleaf scorpionweed & & 1 & 1 & & & & & & & & & & 0.17 \\
\hline 7 & 7-PH-X & Poa bulbosa & bulbous bluegrass & & 28 & & & & & & & & & & & 2.33 \\
\hline 7 & 7-PH-X & Poa sandbergii & Sandberg's bluegrass & 7 & & 3 & 11 & 19 & 28 & 9 & 1 & 2 & & & 4 & 7.00 \\
\hline 7 & 7-PH-X & Ribes aureum & golden currant & & 1 & & & & & & & & & & & 0.08 \\
\hline 7 & 7-PH-X & Sisymbrium altissimum & Jim Hill's tumblemustard & 3 & 3 & 0 & 3 & 10 & 4 & & & & 0 & 2 & & 2.08 \\
\hline 7 & 7-PH-X & Sitanion hystrix & bottlebrush grass & & & & 1 & & & & 2 & 3 & & & 1 & 0.58 \\
\hline
\end{tabular}


Table C.3. Species Found on $10-\mathrm{m}^{2}$ and $100-\mathrm{m}^{2}$ and $1000-\mathrm{m}^{2}$ Modified Whittaker Monitoring Plots

\begin{tabular}{|c|c|c|c|c|c|c|}
\hline $\begin{array}{c}\text { Reveg } \\
\text { Unit }\end{array}$ & Site Name & Genus/Species & Common Name & $1000-\mathrm{m}^{2}$ Species & $100-\mathrm{m}^{2}$ Species & $10-\mathrm{m}^{2}$ Species \\
\hline 0 & 1200-REF-1 & Achillea millefolium & yarrow & 1 & & \\
\hline 0 & 1200-REF-1 & Agropyron spicatum & bluebunch wheatgrass & 1 & 1 & 1 \\
\hline 0 & 1200-REF-1 & Amsinckia lycopsoides & fiddleneck & 1 & 1 & 1 \\
\hline 0 & 1200-REF-1 & Artemisia tridentata & big sagebrush & 1 & 1 & \\
\hline 0 & 1200-REF-1 & Astragalus purshii & woolly-pod milkvetch & 1 & & \\
\hline 0 & 1200-REF-1 & Bromus tectorum & cheatgrass & 1 & 1 & 1 \\
\hline 0 & 1200-REF-1 & Calochortus macrocarpus & sagebrush mariposa lily & 1 & & \\
\hline 0 & 1200-REF-1 & Crepis atribarba & slender hawksbeard & 1 & 1 & 1 \\
\hline 0 & 1200-REF-1 & Epilobium paniculatum & tall willowherb & 1 & 1 & 1 \\
\hline 0 & 1200-REF-1 & Erigeron filifolius & threadleaf fleabane & 1 & 1 & 1 \\
\hline 0 & 1200-REF-1 & Festuca octoflora & slender sixweeks & 1 & 1 & 1 \\
\hline 0 & 1200-REF-1 & Holosteum umbellatum & jagged chickweed & 1 & & \\
\hline 0 & 1200-REF-1 & Lactuca serriola & prickly lettuce & 1 & & \\
\hline 0 & 1200-REF-1 & Lomatium macrocarpum & bigseed desertparsley & 1 & 1 & 1 \\
\hline 0 & 1200-REF-1 & Lupinus sericeus & silky lupine & 1 & 1 & 1 \\
\hline 0 & 1200-REF-1 & Phlox longifolia & longleaf phlox & 1 & 1 & 1 \\
\hline 0 & 1200-REF-1 & Plantago patagonica & indian wheat & 1 & 1 & 1 \\
\hline 0 & 1200-REF-1 & Poa cusickii & Cusick's bluegrass & 1 & 1 & \\
\hline 0 & 1200-REF-1 & Poa sandbergii & Sandberg's bluegrass & 1 & 1 & 1 \\
\hline 0 & 1200-REF-1 & Sisymbrium altissimum & Jim Hill's tumblemustard & 1 & 1 & 1 \\
\hline 0 & 1200-REF-1 & Stipa comata & needle-and-thread grass & 1 & 1 & 1 \\
\hline 0 & 1200-REF-1 & Tragopogon dubius & Yellow salsify & 1 & 1 & \\
\hline 1 & 1-RS-1 & Artemisia tridentata & big sagebrush & & 1 & \\
\hline 1 & 1-RS-1 & Astragalus caricinus & buckwheat milkvetch & & 1 & \\
\hline 1 & 1-RS-1 & Elymus cinereus & giant wildrye & & 1 & 1 \\
\hline 1 & 1-RS-1 & Grayia spinosa & spiny hopsage & & 1 & 1 \\
\hline
\end{tabular}


Table C.3. (contd)

\begin{tabular}{|c|c|c|c|c|c|c|}
\hline $\begin{array}{c}\text { Reveg } \\
\text { Unit }\end{array}$ & Site Name & Genus/Species & Common Name & $1000-\mathrm{m}^{2}$ Species & $100-\mathrm{m}^{2}$ Species & 10-m² Species \\
\hline 1 & 1-RS-1 & Kochia scoparia & summer cypress & & 1 & 1 \\
\hline 1 & 1-RS-1 & Poa sandbergii & Sandberg's bluegrass & & 1 & 1 \\
\hline 1 & 1-RS-1 & Salsola kali & Russian thistle & & 1 & 1 \\
\hline 1 & 1-RS-1 & Sisymbrium altissimum & Jim Hill's tumblemustard & & 1 & \\
\hline 1 & 1-RS-1 & Sitanion hystrix & bottlebrush grass & & 1 & 1 \\
\hline 1 & 1-RS-2 & Artemisia tridentata & big sagebrush & & 1 & \\
\hline 1 & 1-RS-2 & Balsamorhiza careyana & Carey's balsamroot & & 1 & 1 \\
\hline 1 & 1-RS-2 & Bromus tectorum & cheatgrass & & 1 & 1 \\
\hline 1 & 1-RS-2 & Distichlis stricta & alkali saltgrass & & 1 & \\
\hline 1 & 1-RS-2 & Elymus cinereus & giant wildrye & & 1 & 1 \\
\hline 1 & 1-RS-2 & Grayia spinosa & spiny hopsage & & 1 & \\
\hline 1 & 1-RS-2 & Kochia scoparia & summer cypress & & 1 & 1 \\
\hline 1 & 1-RS-2 & Oryzopsis hymenoides & indian ricegrass & & 1 & \\
\hline 1 & 1-RS-2 & Salsola kali & Russian thistle & & 1 & 1 \\
\hline 1 & 1-RS-2 & Sisymbrium altissimum & Jim Hill's tumblemustard & & 1 & \\
\hline 1 & 1-RS-2 & Sitanion hystrix & bottlebrush grass & & 1 & 1 \\
\hline 1 & 1-RS-3 & Achillea millefolium & yarrow & & 1 & 1 \\
\hline 1 & 1-RS-3 & Amsinckia lycopsoides & fiddleneck & & 1 & 1 \\
\hline 1 & 1-RS-3 & Artemisia tridentata & big sagebrush & & 1 & 1 \\
\hline 1 & 1-RS-3 & Balsamorhiza careyana & Carey's balsamroot & & 1 & 1 \\
\hline 1 & 1-RS-3 & Bromus tectorum & cheatgrass & & 1 & \\
\hline 1 & 1-RS-3 & Chenopodium leptophyllum & slimleaf goosefoot & & 1 & 1 \\
\hline 1 & 1-RS-3 & Distichlis stricta & alkali saltgrass & & 1 & 1 \\
\hline 1 & 1-RS-3 & Elymus cinereus & giant wildrye & & 1 & 1 \\
\hline 1 & 1-RS-3 & Grayia spinosa & spiny hopsage & & 1 & 1 \\
\hline 1 & 1-RS-3 & Kochia scoparia & summer cypress & & 1 & 1 \\
\hline
\end{tabular}


Table C.3. (contd)

\begin{tabular}{|c|c|c|c|c|c|c|}
\hline $\begin{array}{c}\text { Reveg } \\
\text { Unit }\end{array}$ & Site Name & Genus/Species & Common Name & $1000-\mathrm{m}^{2}$ Species & $100-\mathrm{m}^{2}$ Species & $10-\mathrm{m}^{2}$ Species \\
\hline 1 & 1-RS-3 & Machaeranthera canescens & hoary aster & & 1 & 1 \\
\hline 1 & $1-\mathrm{RS}-3$ & Poa sandbergii & Sandberg's bluegrass & & 1 & \\
\hline 1 & 1-RS-3 & Salsola kali & Russian thistle & & 1 & \\
\hline 1 & 1-RS-3 & Sisymbrium altissimum & Jim Hill's tumblemustard & & 1 & 1 \\
\hline 1 & 1-RS-3 & Sitanion hystrix & bottlebrush grass & & 1 & \\
\hline 2 & $2-109-1$ & Achillea millefolium & yarrow & & & \\
\hline 2 & $2-109-1$ & Achillea millefolium & yarrow & & 1 & 1 \\
\hline 2 & $2-109-1$ & Agropyron spicatum & bluebunch wheatgrass & & 1 & 1 \\
\hline 2 & $2-109-1$ & Amsinckia tessellata & devil's lettuce & & 1 & \\
\hline 2 & $2-109-1$ & Brodiaea douglasii & Douglas' clusterlily & & 1 & 1 \\
\hline 2 & $2-109-1$ & Bromus tectorum & cheatgrass & & 1 & 1 \\
\hline 2 & $2-109-1$ & Calochortus macrocarpus & sagebrush mariposa lily & & 1 & \\
\hline 2 & $2-109-1$ & Chenopodium leptophyllum & slimleaf goosefoot & & 1 & 1 \\
\hline 2 & $2-109-1$ & Crepis atribarba & slender hawksbeard & & 1 & 1 \\
\hline 2 & $2-109-1$ & Erodium cicutarium & storksbill & & 1 & 1 \\
\hline 2 & $2-109-1$ & Lactuca serriola & prickly lettuce & & 1 & 1 \\
\hline 2 & $2-109-1$ & Linum perenne & wild blueflax & & 1 & 1 \\
\hline 2 & $2-109-1$ & Lupinus sericeus & silky lupine & & 1 & 1 \\
\hline 2 & $2-109-1$ & Machaeranthera canescens & hoary aster & & 1 & 1 \\
\hline 2 & $2-109-1$ & Phlox longifolia & longleaf phlox & & 1 & 1 \\
\hline 2 & $2-109-1$ & Poa bulbosa & bulbous bluegrass & & 1 & 1 \\
\hline 2 & $2-109-1$ & Poa sandbergii & Sandberg's bluegrass & & 1 & 1 \\
\hline 2 & $2-109-1$ & Sisymbrium altissimum & Jim Hill's tumblemustard & & 1 & \\
\hline 2 & $2-109-1$ & Sitanion hystrix & bottlebrush grass & & 1 & \\
\hline 2 & $2-109-1$ & Stipa comata & needle-and-thread grass & & 1 & 1 \\
\hline 2 & $2-109-1$ & Tragopogon dubius & Yellow salsify & & 1 & 1 \\
\hline
\end{tabular}


Table C.3. (contd)

\begin{tabular}{|c|c|c|c|c|c|c|}
\hline $\begin{array}{c}\text { Reveg } \\
\text { Unit }\end{array}$ & Site Name & Genus/Species & Common Name & 1000- $\mathrm{m}^{2}$ Species & $100-\mathrm{m}^{2}$ Species & $10-\mathrm{m}^{2}$ Species \\
\hline 2 & $2-109-2$ & Achillea millefolium & yarrow & & 1 & 1 \\
\hline 2 & $2-109-2$ & Agropyron spicatum & bluebunch wheatgrass & & 1 & \\
\hline 2 & $2-109-2$ & Astragalus caricinus & buckwheat milkvetch & & 1 & 1 \\
\hline 2 & $2-109-2$ & Astragalus purshii & woolly-pod milkvetch & & 1 & \\
\hline 2 & $2-109-2$ & Bromus tectorum & cheatgrass & & 1 & 1 \\
\hline 2 & $2-109-2$ & Calochortus macrocarpus & sagebrush mariposa lily & & 1 & \\
\hline 2 & $2-109-2$ & Crepis atribarba & slender hawksbeard & & 1 & \\
\hline 2 & $2-109-2$ & Erigeron filifolius & threadleaf fleabane & & 1 & \\
\hline 2 & $2-109-2$ & Festuca microstachys & small sixweeks & & 1 & 1 \\
\hline 2 & $2-109-2$ & Lomatium macrocarpum & bigseed desertparsley & & 1 & 1 \\
\hline 2 & $2-109-2$ & Lupinus sericeus & silky lupine & & 1 & 1 \\
\hline 2 & $2-109-2$ & Phlox longifolia & longleaf phlox & & 1 & \\
\hline 2 & $2-109-2$ & Poa bulbosa & bulbous bluegrass & & 1 & \\
\hline 2 & $2-109-2$ & Poa sandbergii & Sandberg’s bluegrass & & 1 & \\
\hline 2 & $2-109-2$ & Sisymbrium altissimum & Jim Hill's tumblemustard & & 1 & \\
\hline 2 & $2-109-2$ & Stipa comata & needle-and-thread grass & & 1 & \\
\hline 2 & $2-109-3$ & Achillea millefolium & yarrow & & 1 & 1 \\
\hline 2 & $2-109-3$ & Agropyron spicatum & bluebunch wheatgrass & & 1 & \\
\hline 2 & $2-109-3$ & Astragalus caricinus & buckwheat milkvetch & & 1 & 1 \\
\hline 2 & $2-109-3$ & Astragalus purshii & woolly-pod milkvetch & & 1 & 1 \\
\hline 2 & $2-109-3$ & Balsamorhiza careyana & Carey's balsamroot & & 1 & 1 \\
\hline 2 & $2-109-3$ & Bromus tectorum & cheatgrass & & 1 & \\
\hline 2 & $2-109-3$ & Calochortus macrocarpus & sagebrush mariposa lily & & 1 & \\
\hline 2 & $2-109-3$ & Crepis atribarba & slender hawksbeard & & 1 & \\
\hline 2 & $2-109-3$ & Epilobium paniculatum & tall willowherb & & 1 & 1 \\
\hline 2 & $2-109-3$ & Erigeron filifolius & threadleaf fleabane & & 1 & 1 \\
\hline
\end{tabular}


Table C.3. (contd)

\begin{tabular}{|c|c|c|c|c|c|c|}
\hline $\begin{array}{c}\text { Reveg } \\
\text { Unit }\end{array}$ & Site Name & Genus/Species & Common Name & 1000- $\mathrm{m}^{2}$ Species & $100-\mathrm{m}^{2}$ Species & $10-\mathrm{m}^{2}$ Species \\
\hline 2 & $2-109-3$ & Erodium cicutarium & storksbill & & 1 & 1 \\
\hline 2 & $2-109-3$ & Festuca microstachys & small sixweeks & & 1 & \\
\hline 2 & $2-109-3$ & Lactuca serriola & prickly lettuce & & 1 & 1 \\
\hline 2 & $2-109-3$ & Linum perenne & wild blueflax & & 1 & \\
\hline 2 & $2-109-3$ & Lomatium macrocarpum & bigseed desertparsley & & 1 & 1 \\
\hline 2 & $2-109-3$ & Lupinus sericeus & silky lupine & & 1 & \\
\hline 2 & $2-109-3$ & Phlox longifolia & longleaf phlox & & 1 & \\
\hline 2 & $2-109-3$ & Poa bulbosa & bulbous bluegrass & & 1 & 1 \\
\hline 2 & $2-109-3$ & Poa sandbergii & Sandberg's bluegrass & & 1 & \\
\hline 2 & $2-109-3$ & Sisymbrium altissimum & Jim Hill's tumblemustard & & 1 & \\
\hline 2 & $2-109-3$ & Stipa comata & needle-and-thread grass & & 1 & 1 \\
\hline 2 & $2-138-1$ & Amsinckia tessellata & devil's lettuce & & 1 & \\
\hline 2 & $2-138-1$ & Artemisia tridentata & big sagebrush & & 1 & \\
\hline 2 & $2-138-1$ & Bromus tectorum & cheatgrass & & 1 & \\
\hline 2 & $2-138-1$ & Chenopodium leptophyllum & slimleaf goosefoot & & 1 & \\
\hline 2 & $2-138-1$ & Crepis atribarba & slender hawksbeard & & 1 & 1 \\
\hline 2 & $2-138-1$ & Lactuca serriola & prickly lettuce & & 1 & \\
\hline 2 & $2-138-1$ & Salsola kali & Russian thistle & & 1 & 1 \\
\hline 2 & $2-138-1$ & Sisymbrium altissimum & Jim Hill's tumblemustard & & 1 & \\
\hline 2 & $2-139-1$ & Achillea millefolium & yarrow & & 1 & 1 \\
\hline 2 & $2-139-1$ & Agropyron spicatum & bluebunch wheatgrass & & 1 & \\
\hline 2 & $2-139-1$ & Amsinckia tessellata & devil's lettuce & & 1 & \\
\hline 2 & $2-139-1$ & Artemisia tridentata & big sagebrush & & 1 & \\
\hline 2 & $2-139-1$ & Bromus tectorum & cheatgrass & & 1 & \\
\hline 2 & $2-139-1$ & Chaenactis douglasii & hoary falseyarrow & & 1 & 1 \\
\hline 2 & $2-139-1$ & Chenopodium leptophyllum & slimleaf goosefoot & & 1 & \\
\hline
\end{tabular}


Table C.3. (contd)

\begin{tabular}{|c|c|c|c|c|c|c|}
\hline $\begin{array}{c}\text { Reveg } \\
\text { Unit }\end{array}$ & Site Name & Genus/Species & Common Name & 1000-m ${ }^{2}$ Species & $100-\mathrm{m}^{2}$ Species & 10- $\mathrm{m}^{2}$ Species \\
\hline 2 & $2-139-1$ & Lactuca serriola & prickly lettuce & & 1 & \\
\hline 2 & $2-139-1$ & Poa sandbergii & Sandberg's bluegrass & & 1 & \\
\hline 2 & $2-139-1$ & Salsola kali & Russian thistle & & 1 & \\
\hline 2 & $2-139-1$ & Sisymbrium altissimum & Jim Hill's tumblemustard & & 1 & \\
\hline 2 & $2-139-1$ & Sitanion hystrix & bottlebrush grass & & 1 & \\
\hline 2 & $2-50-1$ & Achillea millefolium & yarrow & & 1 & \\
\hline 2 & $2-50-1$ & Agropyron spicatum & bluebunch wheatgrass & & 1 & \\
\hline 2 & $2-50-1$ & Amsinckia tessellata & devil's lettuce & & 1 & 1 \\
\hline 2 & $2-50-1$ & Artemisia tridentata & big sagebrush & & 1 & 1 \\
\hline 2 & $2-50-1$ & Bromus tectorum & cheatgrass & & 1 & 1 \\
\hline 2 & $2-50-1$ & Calochortus macrocarpus & sagebrush mariposa lily & & 1 & 1 \\
\hline 2 & $2-50-1$ & Chenopodium leptophyllum & slimleaf goosefoot & & 1 & \\
\hline 2 & $2-50-1$ & Chrysothamnus nauseosus & gray rabbitbrush & & 1 & \\
\hline 2 & $2-50-1$ & Crepis atribarba & slender hawksbeard & & 1 & 1 \\
\hline 2 & $2-50-1$ & Descurainia pinnata & western tansymustard & & 1 & \\
\hline 2 & $2-50-1$ & Erigeron filifolius & threadleaf fleabane & & 1 & \\
\hline 2 & $2-50-1$ & Helianthus cusickii & Cusick's sunflower & & 1 & 1 \\
\hline 2 & $2-50-1$ & Lactuca serriola & prickly lettuce & & 1 & 1 \\
\hline 2 & $2-50-1$ & Linum perenne & wild blueflax & & 1 & \\
\hline 2 & $2-50-1$ & Lupinus sericeus & silky lupine & & 1 & 1 \\
\hline 2 & $2-50-1$ & Machaeranthera canescens & hoary aster & & 1 & 1 \\
\hline 2 & $2-50-1$ & Oryzopsis hymenoides & indian ricegrass & & 1 & 1 \\
\hline 2 & $2-50-1$ & Phlox longifolia & longleaf phlox & & 1 & \\
\hline 2 & $2-50-1$ & Poa sandbergii & Sandberg's bluegrass & & 1 & \\
\hline 2 & $2-50-1$ & Salsola kali & Russian thistle & & 1 & \\
\hline 2 & $2-50-1$ & Sitanion hystrix & bottlebrush grass & & 1 & 1 \\
\hline
\end{tabular}


Table C.3. (contd)

\begin{tabular}{|c|c|c|c|c|c|c|}
\hline $\begin{array}{c}\text { Reveg } \\
\text { Unit }\end{array}$ & Site Name & Genus/Species & Common Name & $1000-\mathrm{m}^{2}$ Species & $100-\mathrm{m}^{2}$ Species & $10-\mathrm{m}^{2}$ Species \\
\hline 2 & $2-50-1$ & Stipa comata & needle-and-thread grass & & 1 & 1 \\
\hline 2 & 2-DS146-X & Achillea millefolium & yarrow & & 1 & \\
\hline 2 & 2-DS146-X & Agropyron spicatum & bluebunch wheatgrass & & 1 & \\
\hline 2 & 2-DS146-X & Amsinckia lycopsoides & fiddleneck & & 1 & 1 \\
\hline 2 & 2-DS146-X & Amsinckia tessellata & devil's lettuce & & 1 & 1 \\
\hline 2 & 2-DS146-X & Artemisia tridentata & big sagebrush & & 1 & \\
\hline 2 & 2-DS146-X & Astragalus caricinus & buckwheat milkvetch & & 1 & 1 \\
\hline 2 & 2-DS146-X & Astragalus succumbens & crouching milkvetch & & 1 & \\
\hline 2 & 2-DS146-X & Bromus tectorum & cheatgrass & & 1 & \\
\hline 2 & 2-DS146-X & Chaenactis douglasii & hoary falseyarrow & & 1 & 1 \\
\hline 2 & 2-DS146-X & Chenopodium leptophyllum & slimleaf goosefoot & & 1 & 1 \\
\hline 2 & 2-DS146-X & Crepis atribarba & slender hawksbeard & & 1 & 1 \\
\hline 2 & 2-DS146-X & Kochia scoparia & summer cypress & & 1 & \\
\hline 2 & 2-DS146-X & Lactuca serriola & prickly lettuce & & 1 & 1 \\
\hline 2 & 2-DS146-X & Lupinus sericeus & silky lupine & & 1 & 1 \\
\hline 2 & 2-DS146-X & Machaeranthera canescens & hoary aster & & 1 & 1 \\
\hline 2 & 2-DS146-X & Phlox longifolia & longleaf phlox & & 1 & \\
\hline 2 & 2-DS146-X & Poa sandbergii & Sandberg's bluegrass & & 1 & \\
\hline 2 & 2-DS146-X & Salsola kali & Russian thistle & & 1 & 1 \\
\hline 2 & 2-DS146-X & Sisymbrium altissimum & Jim Hill's tumblemustard & & 1 & \\
\hline 2 & 2-DS146-X & Sitanion hystrix & bottlebrush grass & & 1 & \\
\hline 2 & 2-DS60-Xa & Achillea millefolium & yarrow & & 1 & \\
\hline 2 & 2-DS60-Xa & Agropyron spicatum & bluebunch wheatgrass & & 1 & \\
\hline 2 & 2-DS60-Xa & Ambrosia acanthicarpa & bur ragweed & & 1 & \\
\hline 2 & 2-DS60-Xa & Amsinckia tessellata & devil's lettuce & & 1 & \\
\hline 2 & 2-DS60-Xa & Artemisia tridentata & big sagebrush & & 1 & 1 \\
\hline
\end{tabular}


Table C.3. (contd)

\begin{tabular}{|c|c|c|c|c|c|c|}
\hline $\begin{array}{c}\text { Reveg } \\
\text { Unit } \\
\end{array}$ & Site Name & Genus/Species & Common Name & $1000-\mathrm{m}^{2}$ Species & $100-m^{2}$ Species & $10-\mathrm{m}^{2}$ Species \\
\hline 2 & 2-DS60-Xa & Chenopodium leptophyllum & slimleaf goosefoot & & 1 & \\
\hline 2 & 2-DS60-Xa & Crepis atribarba & slender hawksbeard & & 1 & 1 \\
\hline 2 & 2-DS60-Xa & Lactuca serriola & prickly lettuce & & 1 & 1 \\
\hline 2 & 2-DS60-Xa & Machaeranthera canescens & hoary aster & & 1 & \\
\hline 2 & 2-DS60-Xa & Phlox longifolia & longleaf phlox & & 1 & 1 \\
\hline 2 & 2-DS60-Xa & Poa ampla Merr. & 'Sherman' big bluegrass & & 1 & 1 \\
\hline 2 & 2-DS60-Xa & Poa sandbergii & Sandberg's bluegrass & & 1 & 1 \\
\hline 2 & 2-DS60-Xa & Salsola kali & Russian thistle & & 1 & \\
\hline 2 & 2-DS60-Xa & Sisymbrium altissimum & Jim Hill's tumblemustard & & 1 & \\
\hline 2 & 2-DS60-Xa & Sitanion hystrix & bottlebrush grass & & 1 & 1 \\
\hline 2 & 2-DS60-Xb & Agropyron spicatum & bluebunch wheatgrass & & 1 & \\
\hline 2 & 2-DS60-Xb & Ambrosia acanthicarpa & bur ragweed & & 1 & \\
\hline 2 & 2-DS60-Xb & Artemisia tridentata & big sagebrush & & 1 & 1 \\
\hline 2 & 2-DS60-Xb & Astragalus succumbens & crouching milkvetch & & 1 & 1 \\
\hline 2 & 2-DS60-Xb & Balsamorhiza careyana & Carey's balsamroot & & 1 & 1 \\
\hline 2 & 2-DS60-Xb & Chenopodium leptophyllum & slimleaf goosefoot & & 1 & \\
\hline 2 & 2-DS60-Xb & Crepis atribarba & slender hawksbeard & & 1 & 1 \\
\hline 2 & 2-DS60-Xb & Erigeron piperianus & Piper's daisy & & 1 & 1 \\
\hline 2 & 2-DS60-Xb & Festuca octoflora & slender sixweeks & & 1 & \\
\hline 2 & 2-DS60-Xb & Helianthus cusickii & Cusick's sunflower & & 1 & 1 \\
\hline 2 & 2-DS60-Xb & Lactuca serriola & prickly lettuce & & 1 & 1 \\
\hline 2 & 2-DS60-Xb & Lupinus sericeus & silky lupine & & 1 & \\
\hline 2 & 2-DS60-Xb & Machaeranthera canescens & hoary aster & & 1 & 1 \\
\hline 2 & 2-DS60-Xb & Poa ampla Merr. & 'Sherman' big bluegrass & & 1 & 1 \\
\hline 2 & 2-DS60-Xb & Poa sandbergii & Sandberg's bluegrass & & 1 & 1 \\
\hline 2 & 2-DS60-Xb & Salsola kali & Russian thistle & & 1 & \\
\hline
\end{tabular}


Table C.3. (contd)

\begin{tabular}{|c|c|c|c|c|c|c|}
\hline $\begin{array}{c}\text { Reveg } \\
\text { Unit }\end{array}$ & Site Name & Genus/Species & Common Name & $1000-\mathrm{m}^{2}$ Species & $100-m^{2}$ Species & $10-\mathrm{m}^{2}$ Species \\
\hline 2 & 2-DS60-Xb & Sisymbrium altissimum & Jim Hill's tumblemustard & & 1 & 1 \\
\hline 2 & 2-DS60-Xb & Sitanion hystrix & bottlebrush grass & & 1 & 1 \\
\hline 2 & 2-HQ-1 & Achillea millefolium & yarrow & & 1 & 1 \\
\hline 2 & 2-HQ-1 & Agoseris grandiflora & showy mountain dandelion & & 1 & \\
\hline 2 & 2-HQ-1 & Agropyron spicatum & bluebunch wheatgrass & & 1 & 1 \\
\hline 2 & 2-HQ-1 & Amsinckia lycopsoides & fiddleneck & & 1 & 1 \\
\hline 2 & 2-HQ-1 & Artemisia tridentata & big sagebrush & & 1 & 1 \\
\hline 2 & 2-HQ-1 & Astragalus caricinus & buckwheat milkvetch & & 1 & \\
\hline 2 & 2-HQ-1 & Astragalus succumbens & crouching milkvetch & & 1 & \\
\hline 2 & 2-HQ-1 & Balsamorhiza careyana & Carey’s balsamroot & & 1 & \\
\hline 2 & 2-HQ-1 & Bromus tectorum & cheatgrass & & 1 & \\
\hline 2 & 2-HQ-1 & Chaenactis douglasii & hoary falseyarrow & & 1 & \\
\hline 2 & 2-HQ-1 & Chrysothamnus nauseosus & gray rabbitbrush & & 1 & \\
\hline 2 & 2-HQ-1 & Erigeron piperianus & Piper's daisy & & 1 & \\
\hline 2 & 2-HQ-1 & Helianthus cusickii & Cusick's sunflower & & 1 & \\
\hline 2 & 2-HQ-1 & Kochia scoparia & summer cypress & & 1 & 1 \\
\hline 2 & 2-HQ-1 & Lactuca serriola & prickly lettuce & & 1 & 1 \\
\hline 2 & 2-HQ-1 & Lupinus sericeus & silky lupine & & 1 & 1 \\
\hline 2 & 2-HQ-1 & Machaeranthera canescens & hoary aster & & 1 & \\
\hline 2 & 2-HQ-1 & Matricaria matricarioides & pineapple weed & & 1 & \\
\hline 2 & 2-HQ-1 & Melilotus alba & white sweetclover & & 1 & \\
\hline 2 & 2-HQ-1 & Phlox longifolia & longleaf phlox & & 1 & 1 \\
\hline 2 & 2-HQ-1 & Poa sandbergii & Sandberg's bluegrass & & 1 & \\
\hline 2 & 2-HQ-1 & Salsola kali & Russian thistle & & 1 & 1 \\
\hline 2 & 2-HQ-1 & Sisymbrium altissimum & Jim Hill's tumblemustard & & 1 & 1 \\
\hline 2 & 2-HQ-1 & Sitanion hystrix & bottlebrush grass & & 1 & 1 \\
\hline
\end{tabular}


Table C.3. (contd)

\begin{tabular}{|c|c|c|c|c|c|c|}
\hline $\begin{array}{c}\text { Reveg } \\
\text { Unit }\end{array}$ & Site Name & Genus/Species & Common Name & $1000-\mathrm{m}^{2}$ Species & $100-m^{2}$ Species & $10-\mathrm{m}^{2}$ Species \\
\hline 2 & 2-HQ-1 & Sporobolus cryptandrus & sand dropseed & & 1 & \\
\hline 2 & 2-HQ-1 & Tragopogon dubius & Yellow salsify & & 1 & \\
\hline 2 & 2-HQ-1 & Vulpia myuros (L.) C.C. Gmel & rat-tail fescue & & 1 & \\
\hline 2 & 2-HQ-2 & Achillea millefolium & yarrow & & 1 & \\
\hline 2 & 2-HQ-2 & Agropyron spicatum & bluebunch wheatgrass & & 1 & \\
\hline 2 & 2-HQ-2 & Amsinckia lycopsoides & fiddleneck & & 1 & \\
\hline 2 & 2-HQ-2 & Artemisia tridentata & big sagebrush & & 1 & \\
\hline 2 & 2-HQ-2 & Astragalus caricinus & buckwheat milkvetch & & 1 & \\
\hline 2 & 2-HQ-2 & Balsamorhiza careyana & Carey's balsamroot & & 1 & \\
\hline 2 & 2-HQ-2 & Bromus tectorum & cheatgrass & & 1 & \\
\hline 2 & 2-HQ-2 & Chorispora tenella & blue mustard & & 1 & \\
\hline 2 & 2-HQ-2 & Lactuca serriola & prickly lettuce & & 1 & \\
\hline 2 & 2-HQ-2 & Melilotus alba & white sweetclover & & 1 & \\
\hline 2 & 2-HQ-2 & Poa sandbergii & Sandberg's bluegrass & & 1 & \\
\hline 2 & 2-HQ-2 & Salsola kali & Russian thistle & & 1 & \\
\hline 2 & 2-HQ-2 & Sisymbrium altissimum & Jim Hill's tumblemustard & & 1 & \\
\hline 2 & 2-HQ-2 & Sitanion hystrix & bottlebrush grass & & 1 & \\
\hline 2 & 2-HQ-2 & Vulpia myuros (L.) C.C. Gmel & rat-tail fescue & & 1 & \\
\hline 2 & 2-HQ-2 & Unknown bunchgrass & Unknown bunchgrass & & 1 & \\
\hline 2 & 2-HQ-3 & Achillea millefolium & yarrow & & 1 & \\
\hline 2 & 2-HQ-3 & Agropyron spicatum & bluebunch wheatgrass & & 1 & \\
\hline 2 & 2-HQ-3 & Amsinckia tessellata & devil's lettuce & & 1 & \\
\hline 2 & 2-HQ-3 & Astragalus caricinus & buckwheat milkvetch & & 1 & \\
\hline 2 & 2-HQ-3 & Balsamorhiza careyana & Carey's balsamroot & & 1 & \\
\hline 2 & 2-HQ-3 & Bromus tectorum & cheatgrass & & 1 & \\
\hline 2 & 2-HQ-3 & Chaenactis douglasii & hoary falseyarrow & & 1 & \\
\hline
\end{tabular}


Table C.3. (contd)

\begin{tabular}{|c|c|c|c|c|c|c|}
\hline $\begin{array}{c}\text { Reveg } \\
\text { Unit }\end{array}$ & Site Name & Genus/Species & Common Name & $1000-\mathrm{m}^{2}$ Species & $100-\mathrm{m}^{2}$ Species & $10-\mathrm{m}^{2}$ Species \\
\hline 2 & $2-\mathrm{HQ}-3$ & Chenopodium leptophyllum & slimleaf goosefoot & & 1 & \\
\hline 2 & 2-HQ-3 & Chorispora tenella & blue mustard & & 1 & \\
\hline 2 & 2-HQ-3 & Lactuca serriola & prickly lettuce & & 1 & \\
\hline 2 & 2-HQ-3 & Lupinus sericeus & silky lupine & & 1 & \\
\hline 2 & 2-HQ-3 & Machaeranthera canescens & hoary aster & & 1 & \\
\hline 2 & 2-HQ-3 & Melilotus alba & white sweetclover & & 1 & \\
\hline 2 & 2-HQ-3 & Poa sandbergii & Sandberg's bluegrass & & 1 & \\
\hline 2 & 2-HQ-3 & Salsola kali & Russian thistle & & 1 & \\
\hline 2 & 2-HQ-3 & Sisymbrium altissimum & Jim Hill's tumblemustard & & 1 & \\
\hline 2 & 2-HQ-3 & Sitanion hystrix & bottlebrush grass & & 1 & \\
\hline 2 & 2-HQ-3 & Triticum $L$. & wheat & & 1 & \\
\hline 2 & 2-HQ-3 & Vulpia myuros (L.) C.C. Gmel & rat-tail fescue & & 1 & \\
\hline 2 & 2-HW-1 & Agropyron spicatum & bluebunch wheatgrass & & 1 & \\
\hline 2 & 2-HW-1 & Amsinckia lycopsoides & fiddleneck & & 1 & \\
\hline 2 & 2-HW-1 & Artemisia tridentata & big sagebrush & & 1 & \\
\hline 2 & 2-HW-1 & Bromus tectorum & cheatgrass & & 1 & \\
\hline 2 & 2-HW-1 & Chenopodium leptophyllum & slimleaf goosefoot & & 1 & \\
\hline 2 & 2-HW-1 & Chondrilla juncea & Rush skeletonweed & & 1 & \\
\hline 2 & 2-HW-1 & Crepis atribarba & slender hawksbeard & & 1 & \\
\hline 2 & 2-HW-1 & Kochia scoparia & summer cypress & & 1 & \\
\hline 2 & 2-HW-1 & Lactuca serriola & prickly lettuce & & 1 & \\
\hline 2 & 2-HW-1 & Machaeranthera canescens & hoary aster & & 1 & \\
\hline 2 & 2-HW-1 & Salsola kali & Russian thistle & & 1 & \\
\hline 2 & 2-HW-1 & Sisymbrium altissimum & Jim Hill's tumblemustard & & 1 & \\
\hline 2 & 2-HW-1 & Sitanion hystrix & bottlebrush grass & & 1 & \\
\hline 2 & 2-HW-1 & Tetradymia canescens & gray horsebrush & & 1 & \\
\hline
\end{tabular}


Table C.3. (contd)

\begin{tabular}{|c|c|c|c|c|c|c|}
\hline $\begin{array}{c}\text { Reveg } \\
\text { Unit }\end{array}$ & Site Name & Genus/Species & Common Name & $1000-\mathrm{m}^{2}$ Species & $100-\mathrm{m}^{2}$ Species & $10-\mathrm{m}^{2}$ Species \\
\hline 2 & $2-\mathrm{HW}-1$ & Tragopogon dubius & Yellow salsify & & 1 & \\
\hline 2 & 2-HW-2 & Achillea millefolium & yarrow & & 1 & 1 \\
\hline 2 & 2-HW-2 & Agropyron spicatum & bluebunch wheatgrass & & 1 & 1 \\
\hline 2 & 2-HW-2 & Amsinckia lycopsoides & fiddleneck & & 1 & \\
\hline 2 & 2-HW-2 & Artemisia tridentata & big sagebrush & & 1 & \\
\hline 2 & 2-HW-2 & Astragalus succumbens & crouching milkvetch & & 1 & 1 \\
\hline 2 & 2-HW-2 & Bromus tectorum & cheatgrass & & 1 & 1 \\
\hline 2 & 2-HW-2 & Chenopodium leptophyllum & slimleaf goosefoot & & 1 & 1 \\
\hline 2 & 2-HW-2 & Chondrilla juncea & Rush skeletonweed & & 1 & 1 \\
\hline 2 & 2-HW-2 & Crepis atribarba & slender hawksbeard & & 1 & 1 \\
\hline 2 & 2-HW-2 & Epilobium paniculatum & tall willowherb & & 1 & 1 \\
\hline 2 & 2-HW-2 & Erodium cicutarium & storksbill & & 1 & 1 \\
\hline 2 & 2-HW-2 & Lactuca serriola & prickly lettuce & & 1 & 1 \\
\hline 2 & 2-HW-2 & Lupinus sericeus & silky lupine & & 1 & 1 \\
\hline 2 & 2-HW-2 & Machaeranthera canescens & hoary aster & & 1 & \\
\hline 2 & 2-HW-2 & Poa sandbergii & Sandberg's bluegrass & & 1 & \\
\hline 2 & 2-HW-2 & Salsola kali & Russian thistle & & 1 & 1 \\
\hline 2 & 2-HW-2 & Sisymbrium altissimum & Jim Hill's tumblemustard & & 1 & 1 \\
\hline 2 & 2-HW-2 & Sitanion hystrix & bottlebrush grass & & 1 & 1 \\
\hline 2 & 2-HW-2 & Tragopogon dubius & Yellow salsify & & 1 & 1 \\
\hline 3 & 3-RT-1 & Agastache occidentalis & western horsemint & & 1 & \\
\hline 3 & 3-RT-1 & Agropyron spicatum & bluebunch wheatgrass & & 1 & \\
\hline 3 & 3-RT-1 & Astragalus purshii & woolly-pod milkvetch & & 1 & \\
\hline 3 & 3-RT-1 & Bromus tectorum & cheatgrass & & 1 & \\
\hline 3 & 3-RT-1 & Chenopodium leptophyllum & slimleaf goosefoot & & 1 & \\
\hline 3 & 3-RT-1 & Crepis modocensis & low hawksbeard & & 1 & \\
\hline
\end{tabular}


Table C.3. (contd)

\begin{tabular}{|c|c|c|c|c|c|c|}
\hline $\begin{array}{c}\text { Reveg } \\
\text { Unit }\end{array}$ & Site Name & Genus/Species & Common Name & 1000- $\mathrm{m}^{2}$ Species & $100-\mathrm{m}^{2}$ Species & $10-\mathrm{m}^{2}$ Species \\
\hline 3 & 3-RT-1 & Festuca idahoensis & Idaho fescue & & 1 & \\
\hline 3 & 3-RT-1 & Lactuca serriola & prickly lettuce & & 1 & \\
\hline 3 & 3-RT-1 & Lomatium grayi & Gray's desertparsley & & 1 & \\
\hline 3 & 3-RT-1 & Lupinus sericeus & silky lupine & & 1 & \\
\hline 3 & 3-RT-1 & Phacelia hastata & whiteleaf scorpionweed & & 1 & \\
\hline 3 & 3-RT-1 & Poa sandbergii & Sandberg's bluegrass & & 1 & \\
\hline 3 & 3-RT-1 & Salsola kali & Russian thistle & & 1 & \\
\hline 3 & 3-RT-1 & Erigeron $S p$. & Erigeron Sp. & & 1 & \\
\hline 3 & 3-RT-1 & Agastache occidentalis & western giant hyssop & & 1 & \\
\hline 3 & 3-RT-2 & Achillea millefolium & yarrow & & 1 & \\
\hline 3 & 3-RT-2 & Agropyron spicatum & bluebunch wheatgrass & & 1 & \\
\hline 3 & $3-\mathrm{RT}-2$ & Bromus tectorum & cheatgrass & & 1 & \\
\hline 3 & 3-RT-2 & Chaenactis douglasii & hoary falseyarrow & & 1 & 1 \\
\hline 3 & $3-\mathrm{RT}-2$ & Chenopodium leptophyllum & slimleaf goosefoot & & 1 & \\
\hline 3 & $3-\mathrm{RT}-2$ & Chrysothamnus nauseosus & gray rabbitbrush & & 1 & \\
\hline 3 & $3-\mathrm{RT}-2$ & Erigeron filifolius & threadleaf fleabane & & 1 & \\
\hline 3 & $3-\mathrm{RT}-2$ & Erysimum asperum & rough wallflower & & 1 & \\
\hline 3 & $3-\mathrm{RT}-2$ & Festuca idahoensis & Idaho fescue & & 1 & \\
\hline 3 & 3-RT-2 & Lactuca serriola & prickly lettuce & & 1 & 1 \\
\hline 3 & $3-\mathrm{RT}-2$ & Lomatium grayi & Gray's desertparsley & & 1 & 1 \\
\hline 3 & 3-RT-2 & Lupinus sericeus & silky lupine & & 1 & \\
\hline 3 & 3-RT-2 & Phacelia hastata & whiteleaf scorpionweed & & 1 & \\
\hline 3 & 3-RT-2 & Poa sandbergii & Sandberg's bluegrass & & 1 & \\
\hline 3 & $3-\mathrm{RT}-2$ & Salsola kali & Russian thistle & & 1 & 1 \\
\hline 3 & $3-\mathrm{RT}-2$ & Sisymbrium altissimum & Jim Hill's tumblemustard & & 1 & \\
\hline 3 & 3-RT-2 & Sitanion hystrix & bottlebrush grass & & 1 & 1 \\
\hline
\end{tabular}


Table C.3. (contd)

\begin{tabular}{|c|c|c|c|c|c|c|}
\hline $\begin{array}{c}\text { Reveg } \\
\text { Unit }\end{array}$ & Site Name & Genus/Species & Common Name & 1000- $\mathrm{m}^{2}$ Species & $100-\mathrm{m}^{2}$ Species & $10-\mathrm{m}^{2}$ Species \\
\hline 3 & 3-RT-2 & Vulpia octoflora (Walter) Rydb. & sixweeks fescue & & 1 & 1 \\
\hline 3 & 3-RT-3 & Achillea millefolium & yarrow & & 1 & \\
\hline 3 & 3-RT-3 & Agropyron spicatum & bluebunch wheatgrass & & 1 & \\
\hline 3 & 3-RT-3 & Balsamorhiza rosea & rosy balsamroot & & 1 & 1 \\
\hline 3 & 3-RT-3 & Bromus japonicus & Japanese brome & & 1 & \\
\hline 3 & 3-RT-3 & Bromus tectorum & cheatgrass & & 1 & 1 \\
\hline 3 & 3-RT-3 & Crepis modocensis & low hawksbeard & & 1 & \\
\hline 3 & 3-RT-3 & Erysimum asperum & rough wallflower & & 1 & \\
\hline 3 & 3-RT-3 & Festuca idahoensis & Idaho fescue & & 1 & 1 \\
\hline 3 & 3-RT-3 & Lomatium grayi & Gray's desertparsley & & 1 & \\
\hline 3 & 3-RT-3 & Phacelia hastata & whiteleaf scorpionweed & & 1 & \\
\hline 3 & 3-RT-3 & Poa sandbergii & Sandberg's bluegrass & & 1 & \\
\hline 3 & 3-RT-3 & Salsola kali & Russian thistle & & 1 & 1 \\
\hline 3 & 3-RT-3 & Sisymbrium altissimum & Jim Hill's tumblemustard & & 1 & \\
\hline 4 & $4-623 \mathrm{~A}-1$ & Agropyron spicatum & bluebunch wheatgrass & & 1 & \\
\hline 4 & $4-623 \mathrm{~A}-1$ & Bromus tectorum & cheatgrass & & 1 & 1 \\
\hline 4 & 4-623A-1 & Festuca idahoensis & Idaho fescue & & 1 & 1 \\
\hline 4 & $4-623 \mathrm{~A}-1$ & Lupinus sericeus & silky lupine & & 1 & 1 \\
\hline 4 & $4-623 \mathrm{~A}-1$ & Phacelia hastata & whiteleaf scorpionweed & & 1 & 1 \\
\hline 4 & $4-623 \mathrm{~A}-1$ & Poa sandbergii & Sandberg's bluegrass & & 1 & \\
\hline 4 & $4-623 \mathrm{~A}-1$ & Polygonum aviculare & doorweed & & 1 & 1 \\
\hline 4 & 4-623A-1 & Salsola kali & Russian thistle & & 1 & 1 \\
\hline 4 & 4-623A-1 & Sisymbrium altissimum & Jim Hill's tumblemustard & & 1 & 1 \\
\hline 4 & $4-623 \mathrm{~A}-1$ & Chenopodium leptophyllum & slimleaf goosefoot & & 1 & 1 \\
\hline 4 & $4-C-1$ & Agropyron spicatum & bluebunch wheatgrass & & 1 & 1 \\
\hline 4 & $4-\mathrm{C}-1$ & Astragalus purshii & woolly-pod milkvetch & & 1 & 1 \\
\hline
\end{tabular}


Table C.3. (contd)

\begin{tabular}{|c|c|c|c|c|c|c|}
\hline $\begin{array}{c}\text { Reveg } \\
\text { Unit }\end{array}$ & Site Name & Genus/Species & Common Name & 1000- $\mathrm{m}^{2}$ Species & $100-\mathrm{m}^{2}$ Species & $10-\mathrm{m}^{2}$ Species \\
\hline 4 & $4-\mathrm{C}-1$ & Erigeron filifolius & threadleaf fleabane & & 1 & 1 \\
\hline 4 & $4-\mathrm{C}-1$ & Festuca idahoensis & Idaho fescue & & 1 & 1 \\
\hline 4 & $4-\mathrm{C}-1$ & Penstemon acuminatus & sand beardtongue & & 1 & 1 \\
\hline 4 & $4-\mathrm{C}-1$ & Phacelia hastata & whiteleaf scorpionweed & & 1 & 1 \\
\hline 4 & $4-\mathrm{C}-1$ & Poa sandbergii & Sandberg's bluegrass & & 1 & \\
\hline 4 & $4-\mathrm{C}-1$ & Salsola kali & Russian thistle & & 1 & \\
\hline 4 & $4-C-1$ & Sisymbrium altissimum & Jim Hill's tumblemustard & & 1 & \\
\hline 4 & $4-C-2$ & Achillea millefolium & yarrow & & 1 & \\
\hline 4 & $4-\mathrm{C}-2$ & Agropyron spicatum & bluebunch wheatgrass & & 1 & \\
\hline 4 & $4-C-2$ & Astragalus purshii & woolly-pod milkvetch & & 1 & 1 \\
\hline 4 & $4-\mathrm{C}-2$ & Bromus tectorum & cheatgrass & & 1 & 1 \\
\hline 4 & $4-C-2$ & Chenopodium leptophyllum & slimleaf goosefoot & & 1 & 1 \\
\hline 4 & $4-\mathrm{C}-2$ & Festuca idahoensis & Idaho fescue & & 1 & \\
\hline 4 & $4-\mathrm{C}-2$ & Lactuca serriola & prickly lettuce & & 1 & \\
\hline 4 & $4-C-2$ & Phacelia hastata & whiteleaf scorpionweed & & 1 & \\
\hline 4 & $4-\mathrm{C}-2$ & Poa sandbergii & Sandberg's bluegrass & & 1 & \\
\hline 4 & $4-\mathrm{C}-2$ & Salsola kali & Russian thistle & & 1 & 1 \\
\hline 4 & $4-\mathrm{C}-2$ & Sisymbrium altissimum & Jim Hill's tumblemustard & & 1 & \\
\hline 4 & $4-C-3$ & Agastache occidentalis & western horsemint & & 1 & 1 \\
\hline 4 & $4-C-3$ & Agropyron spicatum & bluebunch wheatgrass & & 1 & 1 \\
\hline 4 & $4-C-3$ & Astragalus purshii & woolly-pod milkvetch & & 1 & 1 \\
\hline 4 & $4-\mathrm{C}-3$ & Bromus tectorum & cheatgrass & & 1 & \\
\hline 4 & $4-\mathrm{C}-3$ & Chenopodium leptophyllum & slimleaf goosefoot & & 1 & \\
\hline 4 & $4-C-3$ & Erigeron filifolius & threadleaf fleabane & & 1 & 1 \\
\hline 4 & $4-\mathrm{C}-3$ & Erigeron poliospermus & cushion fleabane & & 1 & \\
\hline 4 & $4-\mathrm{C}-3$ & Eriogonum sphaerocephalum & rock buckwheat & & 1 & \\
\hline
\end{tabular}


Table C.3. (contd)

\begin{tabular}{|c|c|c|c|c|c|c|}
\hline $\begin{array}{c}\text { Reveg } \\
\text { Unit }\end{array}$ & Site Name & Genus/Species & Common Name & 1000- $\mathrm{m}^{2}$ Species & $100-\mathrm{m}^{2}$ Species & $10-\mathrm{m}^{2}$ Species \\
\hline 4 & $4-\mathrm{C}-3$ & Festuca idahoensis & Idaho fescue & & 1 & 1 \\
\hline 4 & $4-C-3$ & Lactuca serriola & prickly lettuce & & 1 & 1 \\
\hline 4 & $4-\mathrm{C}-3$ & Phacelia hastata & whiteleaf scorpionweed & & 1 & 1 \\
\hline 4 & $4-C-3$ & Phlox hoodii & Hood's phlox & & 1 & \\
\hline 4 & $4-C-3$ & Poa sandbergii & Sandberg's bluegrass & & 1 & 1 \\
\hline 4 & $4-C-3$ & Salsola kali & Russian thistle & & 1 & 1 \\
\hline 4 & $4-C-3$ & Salvia dorrii & grayball sage & & 1 & \\
\hline 4 & $4-C-3$ & Sisymbrium altissimum & Jim Hill's tumblemustard & & 1 & 1 \\
\hline 4 & 4-CCF-1 & Achillea millefolium & yarrow & & 1 & \\
\hline 4 & 4-CCF-1 & Arenaria franklinii & Franklin's sandwort & & 1 & \\
\hline 4 & 4-CCF-1 & Astragalus purshii & woolly-pod milkvetch & & 1 & 1 \\
\hline 4 & 4-CCF-1 & Balsamorhiza rosea & rosy balsamroot & & 1 & \\
\hline 4 & 4-CCF-1 & Bromus japonicus & Japanese brome & & 1 & \\
\hline 4 & 4-CCF-1 & Bromus tectorum & cheatgrass & & 1 & \\
\hline 4 & 4-CCF-1 & Crepis atribarba & slender hawksbeard & & 1 & \\
\hline 4 & 4-CCF-1 & Crepis modocensis & low hawksbeard & & 1 & \\
\hline 4 & 4-CCF-1 & Eriogonum sphaerocephalum & rock buckwheat & & 1 & \\
\hline 4 & 4-CCF-1 & Eriogonum thymoides & thymeleaf buckwheat & & 1 & \\
\hline 4 & 4-CCF-1 & Lactuca serriola & prickly lettuce & & 1 & \\
\hline 4 & 4-CCF-1 & Lomatium grayi & Gray's desertparsley & & 1 & 1 \\
\hline 4 & 4-CCF-1 & Lomatium macrocarpum & bigseed desertparsley & & 1 & 1 \\
\hline 4 & 4-CCF-1 & Lomatium triternatum & nineleaf desertparsley & & 1 & \\
\hline 4 & 4-CCF-1 & Lupinus sericeus & silky lupine & & 1 & \\
\hline 4 & 4-CCF-1 & Phlox hoodii & Hood's phlox & & 1 & \\
\hline 4 & 4-CCF-1 & Poa sandbergii & Sandberg's bluegrass & & 1 & \\
\hline 4 & 4-CCF-1 & Salsola kali & Russian thistle & & 1 & \\
\hline
\end{tabular}


Table C.3. (contd)

\begin{tabular}{|c|c|c|c|c|c|c|}
\hline $\begin{array}{c}\text { Reveg } \\
\text { Unit }\end{array}$ & Site Name & Genus/Species & Common Name & $1000-\mathrm{m}^{2}$ Species & $100-\mathrm{m}^{2}$ Species & $10-\mathrm{m}^{2}$ Species \\
\hline 4 & 4-CCF-1 & Sisymbrium altissimum & Jim Hill's tumblemustard & & 1 & \\
\hline 4 & 4-CCF-1 & Tragopogon dubius & Yellow salsify & & 1 & \\
\hline 4 & 4-CCF-Berm & Achillea millefolium & yarrow & & 1 & \\
\hline 4 & 4-CCF-Berm & Agropyron spicatum & bluebunch wheatgrass & & 1 & \\
\hline 4 & 4-CCF-Berm & Bromus tectorum & cheatgrass & & 1 & \\
\hline 4 & 4-CCF-Berm & Eriogonum niveum & snow buckwheat & & 1 & \\
\hline 4 & 4-CCF-Berm & Eriophyllum lanatum & woolly sunflower & & 1 & \\
\hline 4 & 4-CCF-Berm & Helianthus cusickii & Cusick's sunflower & & 1 & \\
\hline 4 & 4-CCF-Berm & Lomatium grayi & Gray's desertparsley & & 1 & \\
\hline 4 & 4-CCF-Berm & Melilotus alba & white sweetclover & & 1 & \\
\hline 4 & 4-CCF-Berm & Phacelia hastata & whiteleaf scorpionweed & & 1 & \\
\hline 4 & 4-CCF-Berm & Poa sandbergii & Sandberg's bluegrass & & 1 & \\
\hline 4 & 4-CCF-Berm & Salsola kali & Russian thistle & & 1 & \\
\hline 4 & 4-CCF-Berm & Sisymbrium altissimum & Jim Hill's tumblemustard & & 1 & \\
\hline 4 & 4-CCF-Berm & Chenopodium leptophyllum & slimleaf goosefoot & & 1 & \\
\hline 4 & 4-CCF-road & Achillea millefolium & yarrow & & 1 & 1 \\
\hline 4 & 4-CCF-road & Agropyron spicatum & bluebunch wheatgrass & & 1 & 1 \\
\hline 4 & 4-CCF-road & Antennaria dimorpha & low pussytoes & & 1 & \\
\hline 4 & 4-CCF-road & Balsamorhiza rosea & rosy balsamroot & & 1 & 1 \\
\hline 4 & 4-CCF-road & Bromus japonicus & Japanese brome & & 1 & \\
\hline 4 & 4-CCF-road & Bromus tectorum & cheatgrass & & 1 & 1 \\
\hline 4 & 4-CCF-road & Crepis modocensis & low hawksbeard & & 1 & \\
\hline 4 & 4-CCF-road & Descurainia pinnata & western tansymustard & & 1 & \\
\hline 4 & 4-CCF-road & Eriogonum ovalifolium var. celsum & cushion buckwheat & & 1 & 1 \\
\hline 4 & 4-CCF-road & Eriophyllum lanatum & woolly sunflower & & 1 & \\
\hline 4 & 4-CCF-road & Lomatium grayi & Gray's desertparsley & & 1 & 1 \\
\hline
\end{tabular}


Table C.3. (contd)

\begin{tabular}{|c|c|c|c|c|c|c|}
\hline $\begin{array}{c}\text { Reveg } \\
\text { Unit }\end{array}$ & Site Name & Genus/Species & Common Name & $1000-\mathrm{m}^{2}$ Species & $100-\mathrm{m}^{2}$ Species & $10-\mathrm{m}^{2}$ Species \\
\hline 4 & 4-CCF-road & Lupinus sericeus & silky lupine & & 1 & \\
\hline 4 & 4-CCF-road & $\begin{array}{l}\text { Lygodesmia juncea (Pursh) D. Don ex } \\
\text { Hook. }\end{array}$ & rush skeletonplant & & 1 & 1 \\
\hline 4 & 4-CCF-road & Phacelia hastata & whiteleaf scorpionweed & & 1 & 1 \\
\hline 4 & 4-CCF-road & Poa sandbergii & Sandberg's bluegrass & & 1 & \\
\hline 4 & 4-CCF-road & Polygonum aviculare & doorweed & & 1 & 1 \\
\hline 4 & 4-CCF-road & Salsola kali & Russian thistle & & 1 & \\
\hline 4 & 4-CCF-road & Sisymbrium altissimum & Jim Hill's tumblemustard & & 1 & 1 \\
\hline 5 & $5-\mathrm{OB}-1$ & Agropyron spicatum & bluebunch wheatgrass & & 1 & 1 \\
\hline 5 & $5-\mathrm{OB}-1$ & Bromus tectorum & cheatgrass & & 1 & 1 \\
\hline 5 & $5-\mathrm{OB}-1$ & Chenopodium leptophyllum & slimleaf goosefoot & & 1 & 1 \\
\hline 5 & $5-\mathrm{OB}-1$ & Phacelia hastata & whiteleaf scorpionweed & & 1 & 1 \\
\hline 5 & $5-\mathrm{OB}-1$ & Phlox longifolia & longleaf phlox & & 1 & 1 \\
\hline 5 & $5-\mathrm{OB}-1$ & Poa sandbergii & Sandberg's bluegrass & & 1 & 1 \\
\hline 5 & $5-\mathrm{OB}-1$ & Polygonum aviculare & doorweed & & 1 & 1 \\
\hline 5 & $5-\mathrm{OB}-1$ & Sisymbrium altissimum & Jim Hill's tumblemustard & & 1 & 1 \\
\hline 5 & $5-\mathrm{OB}-2$ & Agropyron spicatum & bluebunch wheatgrass & & 1 & 1 \\
\hline 5 & $5-\mathrm{OB}-2$ & Bromus tectorum & cheatgrass & & 1 & \\
\hline 5 & $5-\mathrm{OB}-2$ & Lomatium grayi & Gray's desertparsley & & 1 & 1 \\
\hline 5 & $5-\mathrm{OB}-2$ & Phacelia hastata & whiteleaf scorpionweed & & 1 & 1 \\
\hline 5 & $5-\mathrm{OB}-2$ & Poa sandbergii & Sandberg's bluegrass & & 1 & 1 \\
\hline 5 & $5-\mathrm{OB}-2$ & Salsola kali & Russian thistle & & 1 & \\
\hline 5 & $5-\mathrm{OB}-2$ & Sisymbrium altissimum & Jim Hill's tumblemustard & & 1 & \\
\hline 6 & $6-6635-\mathrm{X}$ & Achillea millefolium & yarrow & & 1 & 1 \\
\hline 6 & $6-6635-X$ & Agastache occidentalis & western horsemint & & 1 & \\
\hline 6 & $6-6635-X$ & Agropyron spicatum & bluebunch wheatgrass & & 1 & \\
\hline 6 & $6-6635-X$ & Astragalus purshii & woolly-pod milkvetch & & 1 & 1 \\
\hline
\end{tabular}


Table C.3. (contd)

\begin{tabular}{|c|c|c|c|c|c|c|}
\hline $\begin{array}{c}\text { Reveg } \\
\text { Unit }\end{array}$ & Site Name & Genus/Species & Common Name & $1000-\mathrm{m}^{2}$ Species & $100-\mathrm{m}^{2}$ Species & $10-\mathrm{m}^{2}$ Species \\
\hline 6 & $6-6635-X$ & Bromus tectorum & cheatgrass & & 1 & 1 \\
\hline 6 & $6-6635-X$ & Chenopodium leptophyllum & slimleaf goosefoot & & 1 & 1 \\
\hline 6 & $6-6635-X$ & Crepis atribarba & slender hawksbeard & & 1 & 1 \\
\hline 6 & 6-6635-X & Crepis modocensis & low hawksbeard & & 1 & \\
\hline 6 & $6-6635-\mathrm{X}$ & Descurainia pinnata & western tansymustard & & 1 & 1 \\
\hline 6 & $6-6635-X$ & Descurainia sophia & flixweed & & 1 & 1 \\
\hline 6 & $6-6635-\mathrm{X}$ & Erigeron linearis & desert yellowdaisy & & 1 & \\
\hline 6 & $6-6635-X$ & Eriophyllum lanatum & woolly sunflower & & 1 & \\
\hline 6 & $6-6635-X$ & Festuca idahoensis & Idaho fescue & & 1 & \\
\hline 6 & $6-6635-X$ & Lactuca serriola & prickly lettuce & & 1 & 1 \\
\hline 6 & $6-6635-\mathrm{X}$ & Lomatium grayi & Gray's desertparsley & & 1 & 1 \\
\hline 6 & $6-6635-X$ & Lupinus sericeus & silky lupine & & 1 & \\
\hline 6 & $6-6635-\mathrm{X}$ & Phacelia hastata & whiteleaf scorpionweed & & 1 & \\
\hline 6 & $6-6635-X$ & Poa sandbergii & Sandberg's bluegrass & & 1 & 1 \\
\hline 6 & $6-6635-X$ & Polygonum aviculare & doorweed & & 1 & \\
\hline 6 & $6-6635-X$ & Sisymbrium altissimum & Jim Hill's tumblemustard & & 1 & \\
\hline 6 & $6-6635-X$ & Tragopogon dubius & Yellow salsify & & 1 & \\
\hline 6 & 6-6635-X & Lomatium sp. & Lomatium sp. & & 1 & \\
\hline 6 & $6-6636-1$ & Achillea millefolium & Kochia scoparia & & 1 & \\
\hline 6 & $6-6636-1$ & Agastache occidentalis & Lomatium sp. & & 1 & \\
\hline 6 & $6-6636-1$ & Agropyron spicatum & bluebunch wheatgrass & & 1 & \\
\hline 6 & $6-6636-1$ & Astragalus purshii & woolly-pod milkvetch & & 1 & \\
\hline 6 & $6-6636-1$ & Balsamorhiza rosea & rosy balsamroot & & 1 & 1 \\
\hline 6 & $6-6636-1$ & Bromus tectorum & cheatgrass & & 1 & \\
\hline 6 & $6-6636-1$ & Chenopodium leptophyllum & slimleaf goosefoot & & 1 & \\
\hline 6 & $6-6636-1$ & Crepis modocensis & low hawksbeard & & 1 & \\
\hline
\end{tabular}


Table C.3. (contd)

\begin{tabular}{|c|c|c|c|c|c|c|}
\hline $\begin{array}{c}\text { Reveg } \\
\text { Unit }\end{array}$ & Site Name & Genus/Species & Common Name & $1000-\mathrm{m}^{2}$ Species & $100-\mathrm{m}^{2}$ Species & $10-\mathrm{m}^{2}$ Species \\
\hline 6 & $6-6636-1$ & Cymopterus terebithinus & turpentine springparsley & & 1 & 1 \\
\hline 6 & $6-6636-1$ & Descurainia pinnata & western tansymustard & & 1 & 1 \\
\hline 6 & $6-6636-1$ & Erigeron linearis & desert yellowdaisy & & 1 & 1 \\
\hline 6 & $6-6636-1$ & Erigeron poliospermus & cushion fleabane & & 1 & 1 \\
\hline 6 & $6-6636-1$ & Eriogonum sphaerocephalum & rock buckwheat & & 1 & 1 \\
\hline 6 & $6-6636-1$ & Festuca idahoensis & Idaho fescue & & 1 & \\
\hline 6 & 6-6636-1 & Haplopappus stenophylla & narrowleaf goldenweed & & 1 & \\
\hline 6 & 6-6636-1 & Lupinus sericeus & silky lupine & & 1 & \\
\hline 6 & $6-6636-1$ & Phacelia hastata & whiteleaf scorpionweed & & 1 & \\
\hline 6 & $6-6636-1$ & Poa sandbergii & Sandberg's bluegrass & & 1 & \\
\hline 6 & $6-6636-1$ & Ribes aureum & golden currant & & 1 & \\
\hline 6 & $6-6636-1$ & Salvia dorrii & grayball sage & & 1 & 1 \\
\hline 6 & $6-6636-1$ & Sisymbrium altissimum & Jim Hill's tumblemustard & & 1 & \\
\hline 6 & $6-6636-1$ & Sitanion hystrix & bottlebrush grass & & 1 & 1 \\
\hline 6 & $6-6636-1$ & Kochia scoparia & Kochia scoparia & & 1 & \\
\hline 7 & 7-PH-X & Achillea millefolium & yarrow & & 1 & \\
\hline 7 & 7-PH-X & Agastache occidentalis & western horsemint & & 1 & 1 \\
\hline 7 & 7-PH-X & Agropyron spicatum & bluebunch wheatgrass & & 1 & 1 \\
\hline 7 & 7-PH-X & Artemisia tridentata & big sagebrush & & 1 & 1 \\
\hline 7 & 7-PH-X & Astragalus purshii & woolly-pod milkvetch & & 1 & 1 \\
\hline 7 & 7-PH-X & Bromus tectorum & cheatgrass & & 1 & \\
\hline 7 & 7-PH-X & Chenopodium leptophyllum & slimleaf goosefoot & & 1 & 1 \\
\hline 7 & 7-PH-X & Chrysothamnus nauseosus & gray rabbitbrush & & 1 & 1 \\
\hline 7 & 7-PH-X & Clematis ligustifolia & western virginsbower & & 1 & 1 \\
\hline 7 & 7-PH-X & Descurainia pinnata & western tansymustard & & 1 & 1 \\
\hline 7 & 7-PH-X & Elymus cinereus & giant wildrye & & 1 & 1 \\
\hline
\end{tabular}


Table C.3. (contd)

\begin{tabular}{|c|c|c|c|c|c|c|}
\hline $\begin{array}{c}\text { Reveg } \\
\text { Unit }\end{array}$ & Site Name & Genus/Species & Common Name & $1000-\mathrm{m}^{2}$ Species & $100-\mathrm{m}^{2}$ Species & $10-\mathrm{m}^{2}$ Species \\
\hline 7 & 7-PH-X & Lactuca serriola & prickly lettuce & & 1 & \\
\hline 7 & 7-PH-X & Phacelia hastata & whiteleaf scorpionweed & & 1 & \\
\hline 7 & 7-PH-X & Poa sandbergii & Sandberg's bluegrass & & 1 & 1 \\
\hline 7 & 7-PH-X & Polygonum aviculare & doorweed & & 1 & 1 \\
\hline 7 & 7-PH-X & Prunus virginiana & chokecherry & & 1 & 1 \\
\hline 7 & 7-PH-X & Ribes aureum & golden currant & & 1 & 1 \\
\hline 7 & 7-PH-X & Sisymbrium altissimum & Jim Hill's tumblemustard & & 1 & \\
\hline 7 & 7-PH-X & Sitanion hystrix & bottlebrush grass & & 1 & 1 \\
\hline 7 & 7-PH-X & Tragopogon dubius & Yellow salsify & & 1 & \\
\hline 7 & 7-PH-X & Verbascum thapsus & common mullein & & 1 & 1 \\
\hline 7 & 7-PH-X & Lomatium sp. & Lomatium sp. & & 1 & 1 \\
\hline 7 & 7-PH-X & Verbena bracteata & bigbract verbena & & 1 & 1 \\
\hline 0 & RR-1 & Achillea millefolium & yarrow & 1 & 1 & 1 \\
\hline 0 & RR-1 & Agropyron spicatum & bluebunch wheatgrass & 1 & 1 & 1 \\
\hline 0 & RR-1 & Antennaria dimorpha & low pussytoes & 1 & 1 & \\
\hline 0 & RR-1 & Astragalus purshii & woolly-pod milkvetch & 1 & 1 & \\
\hline 0 & RR-1 & Balsamorhiza rosea & rosy balsamroot & 1 & 1 & \\
\hline 0 & RR-1 & Bromus tectorum & cheatgrass & 1 & 1 & \\
\hline 0 & RR-1 & Castilleja thompsonii & Thompson's paintbrush & 1 & 1 & \\
\hline 0 & RR-1 & Crepis modocensis & low hawksbeard & 1 & 1 & 1 \\
\hline 0 & RR-1 & Erigeron linearis & desert yellowdaisy & 1 & 1 & 1 \\
\hline 0 & RR-1 & Erigeron poliospermus & cushion fleabane & 1 & 1 & 1 \\
\hline 0 & RR-1 & Eriogonum sphaerocephalum & rock buckwheat & 1 & 1 & 1 \\
\hline 0 & RR-1 & Eriogonum thymoides & thymeleaf buckwheat & 1 & 1 & 1 \\
\hline 0 & RR-1 & Festuca idahoensis & Idaho fescue & 1 & 1 & 1 \\
\hline 0 & RR-1 & Haplopappus stenophylla & narrowleaf goldenweed & 1 & 1 & 1 \\
\hline
\end{tabular}


Table C.3. (contd)

\begin{tabular}{|c|c|c|c|c|c|c|}
\hline $\begin{array}{c}\text { Reveg } \\
\text { Unit }\end{array}$ & Site Name & Genus/Species & Common Name & $1000-\mathrm{m}^{2}$ Species & $100-\mathrm{m}^{2}$ Species & $10-\mathrm{m}^{2}$ Species \\
\hline 0 & RR-1 & Lupinus sericeus & silky lupine & 1 & 1 & \\
\hline 0 & RR-1 & Phacelia hastata & whiteleaf scorpionweed & 1 & 1 & \\
\hline 0 & RR-1 & Phlox hoodii & Hood's phlox & 1 & 1 & \\
\hline 0 & RR-1 & Poa sandbergii & Sandberg's bluegrass & 1 & 1 & \\
\hline 0 & RR-1 & Salvia dorrii & grayball sage & 1 & 1 & \\
\hline
\end{tabular}


Table C.4. Initial Results Measured in Summer 2011 for Percent Survival of Plants Seeded and Transplanted by the Confederated Tribes of the Umatilla Indian Reservation on ALE Revegetation Units

\begin{tabular}{|c|c|c|c|c|c|c|}
\hline $\begin{array}{c}\text { Revegetation } \\
\text { Unit }\end{array}$ & Plot ID & Genus and Species & Common Name & $\begin{array}{c}\text { Plant }(\mathrm{p}) \text { or } \\
\text { Seed }(\mathrm{s})\end{array}$ & $\begin{array}{l}\text { Number } \\
\text { Planted }\end{array}$ & $\%$ Survival \\
\hline 2 & 280 & Achnatherum thurberianum & Thurber's needlegrass & $\mathrm{p}$ & 7 & 42.86 \\
\hline 2 & 281 & Achnatherum thurberianum & Thurber's needlegrass & $\mathrm{p}$ & 7 & 14.29 \\
\hline 2 & 283 & Achnatherum thurberianum & Thurber's needlegrass & $\mathrm{p}$ & 7 & 57.14 \\
\hline 3 & 65 & Agastache occidentalis & western horsemint & $\mathrm{p}$ & 24 & 95.83 \\
\hline 3 & 1589 & Agastache occidentalis & western horsemint & $\mathrm{s}$ & 517 & 1.161 \\
\hline 4 & 64 & Agastache occidentalis & western horsemint & $\mathrm{p}$ & 24 & 50 \\
\hline 4 & 260 & Agastache occidentalis & western horsemint & $\mathrm{s}$ & 50 & 0 \\
\hline 4 & 261 & Agastache occidentalis & western horsemint & $\mathrm{s}$ & 50 & 0 \\
\hline 4 & 262 & Agastache occidentalis & western horsemint & $\mathrm{s}$ & 50 & 0 \\
\hline 4 & 263 & Agastache occidentalis & western horsemint & $\mathrm{s}$ & 50 & 0 \\
\hline 4 & 264 & Agastache occidentalis & western horsemint & $\mathrm{s}$ & 50 & 0 \\
\hline 4 & 265 & Agastache occidentalis & western horsemint & $\mathrm{s}$ & 50 & 0 \\
\hline 4 & 269 & Agastache occidentalis & western horsemint & $\mathrm{s}$ & 50 & 0 \\
\hline 4 & 270 & Agastache occidentalis & western horsemint & $\mathrm{s}$ & 50 & 0 \\
\hline 4 & 272 & Agastache occidentalis & western horsemint & $\mathrm{s}$ & 50 & 0 \\
\hline 4 & 273 & Agastache occidentalis & western horsemint & $\mathrm{s}$ & 50 & 0 \\
\hline 4 & 274 & Agastache occidentalis & western horsemint & $\mathrm{s}$ & 50 & 0 \\
\hline 4 & 275 & Agastache occidentalis & western horsemint & $\mathrm{s}$ & 50 & 0 \\
\hline 4 & 276 & Agastache occidentalis & western horsemint & $\mathrm{s}$ & 50 & 0 \\
\hline 4 & 284 & Agastache occidentalis & western horsemint & $\mathrm{p}$ & 24 & 75 \\
\hline 4 & 285 & Agastache occidentalis & western horsemint & $\mathrm{p}$ & 24 & 83.33 \\
\hline 4 & 286 & Agastache occidentalis & western horsemint & $\mathrm{p}$ & 24 & 70.83 \\
\hline 4 & 287 & Agastache occidentalis & western horsemint & $\mathrm{p}$ & 24 & 95.83 \\
\hline 5 & 266 & Agastache occidentalis & western horsemint & $\mathrm{S}$ & 50 & 0 \\
\hline 5 & 267 & Agastache occidentalis & western horsemint & $\mathrm{s}$ & 50 & 0 \\
\hline 5 & 268 & Agastache occidentalis & western horsemint & $\mathrm{S}$ & 50 & 0 \\
\hline
\end{tabular}


Table C.4. (contd)

\begin{tabular}{|c|c|c|c|c|c|c|}
\hline $\begin{array}{l}\text { Revegetation } \\
\text { Unit }\end{array}$ & Plot ID & Genus and Species & Common Name & $\begin{array}{l}\text { Plant (p) or } \\
\text { Seed (s) }\end{array}$ & $\begin{array}{l}\text { Number } \\
\text { Planted }\end{array}$ & $\%$ Survival \\
\hline 6 & 66 & Agastache occidentalis & western horsemint & $\mathrm{p}$ & 24 & 91.67 \\
\hline 6 & 278 & Agastache occidentalis & western horsemint & s & 50 & 0 \\
\hline 6 & 279 & Agastache occidentalis & western horsemint & $\mathrm{s}$ & 50 & 0 \\
\hline 6 & 1590 & Agastache occidentalis & western horsemint & $\mathrm{p}$ & 24 & 91.67 \\
\hline 1 & Circle & Agoseris grandiflora & showy mountain dandelion & $\mathrm{p}$ & 12 & 91.67 \\
\hline 1 & Road & Agoseris grandiflora & showy mountain dandelion & $\mathrm{p}$ & 13 & 76.92 \\
\hline 2 & 280 & Agoseris grandiflora & showy mountain dandelion & $\mathrm{p}$ & 21 & 71.43 \\
\hline 2 & 281 & Agoseris grandiflora & showy mountain dandelion & $\mathrm{p}$ & 21 & 80.95 \\
\hline 2 & 282 & Agoseris grandiflora & showy mountain dandelion & $\mathrm{p}$ & 21 & 90.48 \\
\hline 1 & 1581 & Artemisia tridentata & big sagebrush & $\mathrm{s}$ & 1000 & 0 \\
\hline 1 & 1582 & Artemisia tridentata & big sagebrush & $\mathrm{s}$ & 882 & 0 \\
\hline 1 & 1583 & Artemisia tridentata & big sagebrush & $\mathrm{s}$ & 824 & 0 \\
\hline 2 & gas well rd & Artemisia tridentata & big sagebrush & $\mathrm{p}$ & 134 & 47.76 \\
\hline 3 & 1589 & Artemisia tridentata & big sagebrush & $\mathrm{s}$ & 929 & 0.108 \\
\hline 1 & Circle & Astragalus caricinus & buckwheat milkvetch & $\mathrm{p}$ & 12 & 16.67 \\
\hline 1 & Road & Astragalus caricinus & buckwheat milkvetch & $\mathrm{p}$ & 13 & 92.31 \\
\hline 2 & 280 & Astragalus caricinus & buckwheat milkvetch & $\mathrm{p}$ & 25 & 56 \\
\hline 2 & 281 & Astragalus caricinus & buckwheat milkvetch & $\mathrm{p}$ & 25 & 56 \\
\hline 2 & 282 & Astragalus caricinus & buckwheat milkvetch & $\mathrm{p}$ & 25 & 88 \\
\hline 2 & 283 & Astragalus caricinus & buckwheat milkvetch & $\mathrm{p}$ & 25 & 60 \\
\hline
\end{tabular}


Table C.4. (contd)

\begin{tabular}{|c|c|c|c|c|c|c|}
\hline $\begin{array}{c}\text { Revegetation } \\
\text { Unit }\end{array}$ & Plot ID & Genus and Species & Common Name & $\begin{array}{l}\text { Plant (p) or } \\
\text { Seed (s) }\end{array}$ & $\begin{array}{l}\text { Number } \\
\text { Planted }\end{array}$ & $\%$ Survival \\
\hline 3 & 65 & Astragalus purshii & woolly-pod milkvetch & $\mathrm{p}$ & 35 & 71.43 \\
\hline 3 & 1589 & Astragalus purshii & woolly-pod milkvetch & $\mathrm{s}$ & 794 & 0 \\
\hline 4 & 64 & Astragalus purshii & woolly-pod milkvetch & $\mathrm{p}$ & 35 & 80 \\
\hline 4 & 260 & Astragalus purshii & woolly-pod milkvetch & $\mathrm{s}$ & 50 & 0 \\
\hline 4 & 261 & Astragalus purshii & woolly-pod milkvetch & $\mathrm{s}$ & 50 & 0 \\
\hline 4 & 262 & Astragalus purshii & woolly-pod milkvetch & $\mathrm{s}$ & 50 & 0 \\
\hline 4 & 263 & Astragalus purshii & woolly-pod milkvetch & $\mathrm{s}$ & 50 & 0 \\
\hline 4 & 264 & Astragalus purshii & woolly-pod milkvetch & $\mathrm{s}$ & 50 & 0 \\
\hline 4 & 265 & Astragalus purshii & woolly-pod milkvetch & $\mathrm{s}$ & 50 & 0 \\
\hline 4 & 269 & Astragalus purshii & woolly-pod milkvetch & $\mathrm{s}$ & 50 & 0 \\
\hline 4 & 270 & Astragalus purshii & woolly-pod milkvetch & $\mathrm{s}$ & 50 & 0 \\
\hline 4 & 272 & Astragalus purshii & woolly-pod milkvetch & $\mathrm{s}$ & 50 & 0 \\
\hline 4 & 273 & Astragalus purshii & woolly-pod milkvetch & $\mathrm{s}$ & 50 & 0 \\
\hline 4 & 274 & Astragalus purshii & woolly-pod milkvetch & $\mathrm{s}$ & 50 & 2 \\
\hline 4 & 275 & Astragalus purshii & woolly-pod milkvetch & $\mathrm{s}$ & 50 & 0 \\
\hline 4 & 276 & Astragalus purshii & woolly-pod milkvetch & $\mathrm{s}$ & 50 & 0 \\
\hline 4 & 284 & Astragalus purshii & woolly-pod milkvetch & $\mathrm{p}$ & 29 & 79.31 \\
\hline 4 & 285 & Astragalus purshii & woolly-pod milkvetch & $\mathrm{p}$ & 29 & 68.97 \\
\hline 4 & 286 & Astragalus purshii & woolly-pod milkvetch & $\mathrm{p}$ & 35 & 74.29 \\
\hline 4 & 287 & Astragalus purshii & woolly-pod milkvetch & $\mathrm{p}$ & 35 & 51.43 \\
\hline 4 & 1592 & Astragalus purshii & woolly-pod milkvetch & $\mathrm{p}$ & 12 & 83.33 \\
\hline 5 & 266 & Astragalus purshii & woolly-pod milkvetch & $\mathrm{s}$ & 50 & 0 \\
\hline 5 & 267 & Astragalus purshii & woolly-pod milkvetch & $\mathrm{s}$ & 50 & 0 \\
\hline 5 & 268 & Astragalus purshii & woolly-pod milkvetch & $\mathrm{s}$ & 50 & 0 \\
\hline 5 & 1798 & Astragalus purshii & woolly-pod milkvetch & $\mathrm{p}$ & 33 & 30.3 \\
\hline 6 & 66 & Astragalus purshii & woolly-pod milkvetch & $\mathrm{p}$ & 35 & 80 \\
\hline 6 & 277 & Astragalus purshii & woolly-pod milkvetch & $\mathrm{s}$ & 50 & 0 \\
\hline 6 & 278 & Astragalus purshii & woolly-pod milkvetch & $\mathrm{s}$ & 50 & 0 \\
\hline
\end{tabular}


Table C.4. (contd)

\begin{tabular}{|c|c|c|c|c|c|c|}
\hline $\begin{array}{l}\text { Revegetation } \\
\text { Unit }\end{array}$ & Plot ID & Genus and Species & Common Name & $\begin{array}{l}\text { Plant (p) or } \\
\text { Seed (s) }\end{array}$ & $\begin{array}{l}\text { Number } \\
\text { Planted }\end{array}$ & $\%$ Survival \\
\hline 6 & 279 & Astragalus purshii & woolly-pod milkvetch & $\mathrm{s}$ & 50 & 0 \\
\hline 6 & 1590 & Astragalus purshii & woolly-pod milkvetch & $\mathrm{p}$ & 35 & 77.14 \\
\hline 6 & 1591 & Astragalus purshii & woolly-pod milkvetch & $\mathrm{p}$ & 37 & 89.19 \\
\hline 7 & 288 & Astragalus purshii & woolly-pod milkvetch & $\mathrm{p}$ & 12 & 41.67 \\
\hline 1 & 1581 & Astragalus succumbens & crouching milkvetch & $\mathrm{s}$ & 1094 & 0 \\
\hline 1 & 1582 & Astragalus succumbens & crouching milkvetch & s & 988 & 0 \\
\hline 1 & 1583 & Astragalus succumbens & crouching milkvetch & $\mathrm{s}$ & 1074 & 0.466 \\
\hline 2 & 1568 & Astragalus succumbens & crouching milkvetch & s & 467 & 0.214 \\
\hline 2 & 1569 & Astragalus succumbens & crouching milkvetch & $\mathrm{s}$ & 463 & 6.695 \\
\hline 3 & 65 & Balsamorhiza rosea & rosy balsamroot & $\mathrm{p}$ & 2 & 0 \\
\hline 4 & 64 & Balsamorhiza rosea & rosy balsamroot & $\mathrm{p}$ & 2 & 0 \\
\hline 4 & 260 & Balsamorhiza rosea & rosy balsamroot & $\mathrm{s}$ & 50 & 0 \\
\hline 4 & 261 & Balsamorhiza rosea & rosy balsamroot & $\mathrm{s}$ & 50 & 0 \\
\hline 4 & 262 & Balsamorhiza rosea & rosy balsamroot & $\mathrm{s}$ & 50 & 0 \\
\hline 4 & 263 & Balsamorhiza rosea & rosy balsamroot & $\mathrm{s}$ & 50 & 2 \\
\hline 4 & 264 & Balsamorhiza rosea & rosy balsamroot & s & 50 & 0 \\
\hline 4 & 265 & Balsamorhiza rosea & rosy balsamroot & s & 50 & 0 \\
\hline 4 & 269 & Balsamorhiza rosea & rosy balsamroot & $\mathrm{s}$ & 50 & 0 \\
\hline 4 & 270 & Balsamorhiza rosea & rosy balsamroot & s & 50 & 0 \\
\hline 4 & 274 & Balsamorhiza rosea & rosy balsamroot & $\mathrm{s}$ & 50 & 2 \\
\hline 4 & 284 & Balsamorhiza rosea & rosy balsamroot & $\mathrm{p}$ & 2 & 100 \\
\hline 4 & 285 & Balsamorhiza rosea & rosy balsamroot & $\mathrm{p}$ & 2 & 100 \\
\hline 4 & 286 & Balsamorhiza rosea & rosy balsamroot & $\mathrm{p}$ & 2 & 0 \\
\hline 4 & 287 & Balsamorhiza rosea & rosy balsamroot & $\mathrm{p}$ & 2 & 50 \\
\hline 5 & 266 & Balsamorhiza rosea & rosy balsamroot & $\mathrm{s}$ & 50 & 2 \\
\hline 5 & 267 & Balsamorhiza rosea & rosy balsamroot & $\mathrm{s}$ & 50 & 0 \\
\hline 5 & 268 & Balsamorhiza rosea & rosy balsamroot & s & 50 & 2 \\
\hline 5 & 1798 & Balsamorhiza rosea & rosy balsamroot & $\mathrm{p}$ & 2 & 50 \\
\hline
\end{tabular}


Table C.4. (contd)

\begin{tabular}{|c|c|c|c|c|c|c|}
\hline $\begin{array}{c}\text { Revegetation } \\
\text { Unit }\end{array}$ & Plot ID & Genus and Species & Common Name & $\begin{array}{l}\text { Plant (p) or } \\
\text { Seed (s) }\end{array}$ & $\begin{array}{l}\text { Number } \\
\text { Planted }\end{array}$ & $\%$ Survival \\
\hline 6 & 66 & Balsamorhiza rosea & rosy balsamroot & $\mathrm{p}$ & 1 & 0 \\
\hline 6 & 1590 & Balsamorhiza rosea & rosy balsamroot & $\mathrm{p}$ & 1 & 0 \\
\hline 6 & 1591 & Balsamorhiza rosea & rosy balsamroot & $\mathrm{p}$ & 2 & 100 \\
\hline 2 & 1568 & Calochortus macrocarpus & sagebrush mariposa lily & $\mathrm{s}$ & 997 & 0 \\
\hline 2 & 1569 & Calochortus macrocarpus & sagebrush mariposa lily & $\mathrm{s}$ & 1046 & 0 \\
\hline 2 & 1571 & Calochortus macrocarpus & sagebrush mariposa lily & $\mathrm{s}$ & 1364 & 0 \\
\hline 2 & 1572 & Calochortus macrocarpus & sagebrush mariposa lily & $\mathrm{s}$ & 1233 & 0 \\
\hline 2 & 1573 & Calochortus macrocarpus & sagebrush mariposa lily & $\mathrm{s}$ & 1253 & 0 \\
\hline 2 & 1574 & Calochortus macrocarpus & sagebrush mariposa lily & $\mathrm{s}$ & 1247 & 0 \\
\hline 2 & 1575 & Calochortus macrocarpus & sagebrush mariposa lily & $\mathrm{s}$ & 1309 & 0 \\
\hline 2 & 1576 & Calochortus macrocarpus & sagebrush mariposa lily & $\mathrm{s}$ & 1316 & 0 \\
\hline 2 & 1577 & Calochortus macrocarpus & sagebrush mariposa lily & $\mathrm{s}$ & 1316 & 0 \\
\hline 2 & 1578 & Calochortus macrocarpus & sagebrush mariposa lily & $\mathrm{s}$ & 1281 & 0 \\
\hline 2 & 1579 & Calochortus macrocarpus & sagebrush mariposa lily & $\mathrm{s}$ & 1330 & 0 \\
\hline 2 & 1580 & Calochortus macrocarpus & sagebrush mariposa lily & $\mathrm{s}$ & 1399 & 0 \\
\hline 2 & 1584 & Calochortus macrocarpus & sagebrush mariposa lily & $\mathrm{s}$ & 1157 & 0 \\
\hline 2 & 1585 & Calochortus macrocarpus & sagebrush mariposa lily & $\mathrm{s}$ & 1489 & 0 \\
\hline 2 & 1586 & Calochortus macrocarpus & sagebrush mariposa lily & $\mathrm{s}$ & 1558 & 0 \\
\hline 2 & 1587 & Calochortus macrocarpus & sagebrush mariposa lily & $\mathrm{s}$ & 1259 & 0 \\
\hline 2 & 1588 & Calochortus macrocarpus & sagebrush mariposa lily & $\mathrm{s}$ & 1316 & 0 \\
\hline 1 & 1581 & Chaenactis douglasii & hoary falseyarrow & $\mathrm{s}$ & 914 & 0 \\
\hline 1 & 1582 & Chaenactis douglasii & hoary falseyarrow & $\mathrm{s}$ & 820 & 0.366 \\
\hline 1 & 1583 & Chaenactis douglasii & hoary falseyarrow & $\mathrm{s}$ & 755 & 1.325 \\
\hline 2 & 1568 & Chaenactis douglasii & hoary falseyarrow & $\mathrm{s}$ & 260 & 5 \\
\hline 2 & 1569 & Chaenactis douglasii & hoary falseyarrow & $\mathrm{s}$ & 190 & 11.05 \\
\hline 2 & 1571 & Chaenactis douglasii & hoary falseyarrow & $\mathrm{s}$ & 176 & 17.05 \\
\hline 2 & 1572 & Chaenactis douglasii & hoary falseyarrow & $\mathrm{s}$ & 256 & 7.812 \\
\hline 2 & 1573 & Chaenactis douglasii & hoary falseyarrow & $\mathrm{s}$ & 251 & 9.562 \\
\hline
\end{tabular}


Table C.4. (contd)

\begin{tabular}{|c|c|c|c|c|c|c|}
\hline $\begin{array}{l}\text { Revegetation } \\
\text { Unit }\end{array}$ & Plot ID & Genus and Species & Common Name & $\begin{array}{l}\text { Plant (p) or } \\
\text { Seed (s) }\end{array}$ & $\begin{array}{l}\text { Number } \\
\text { Planted }\end{array}$ & $\%$ Survival \\
\hline 2 & 1574 & Chaenactis douglasii & hoary falseyarrow & $\mathrm{s}$ & 216 & 9.722 \\
\hline 2 & 1575 & Chaenactis douglasii & hoary falseyarrow & $\mathrm{s}$ & 348 & 0.575 \\
\hline 2 & 1576 & Chaenactis douglasii & hoary falseyarrow & $\mathrm{s}$ & 696 & 1.724 \\
\hline 2 & 1577 & Chaenactis douglasii & hoary falseyarrow & s & 736 & 2.038 \\
\hline 2 & 1578 & Chaenactis douglasii & hoary falseyarrow & $\mathrm{s}$ & 767 & 3.52 \\
\hline 2 & 1579 & Chaenactis douglasii & hoary falseyarrow & $\mathrm{s}$ & 767 & 3.651 \\
\hline 2 & 1580 & Chaenactis douglasii & hoary falseyarrow & $\mathrm{s}$ & 754 & 6.631 \\
\hline 2 & 1584 & Chaenactis douglasii & hoary falseyarrow & s & 754 & 0.663 \\
\hline 2 & 1585 & Chaenactis douglasii & hoary falseyarrow & $\mathrm{s}$ & 705 & 4.681 \\
\hline 2 & 1586 & Chaenactis douglasii & hoary falseyarrow & s & 890 & 2.584 \\
\hline 2 & 1588 & Chaenactis douglasii & hoary falseyarrow & $\mathrm{s}$ & 802 & 0 \\
\hline 1 & 1581 & Clematis ligustifolia & western virginsbower & $\mathrm{s}$ & 92 & 0 \\
\hline 1 & 1582 & Clematis ligustifolia & western virginsbower & $\mathrm{s}$ & 92 & 0 \\
\hline 1 & 1583 & Clematis ligustifolia & western virginsbower & $\mathrm{s}$ & 92 & 0 \\
\hline 7 & 288 & Clematis ligustifolia & western virginsbower & $\mathrm{p}$ & 6 & 0 \\
\hline 1 & 1581 & Crepis atribarba & slender hawksbeard & s & 2985 & 0 \\
\hline 1 & 1582 & Crepis atribarba & slender hawksbeard & s & 2941 & 0 \\
\hline 1 & 1583 & Crepis atribarba & slender hawksbeard & $\mathrm{s}$ & 2956 & 0 \\
\hline 2 & 1568 & Crepis atribarba & slender hawksbeard & s & 500 & 0 \\
\hline 2 & 1569 & Crepis atribarba & slender hawksbeard & $\mathrm{s}$ & 544 & 0 \\
\hline 2 & 1571 & Crepis atribarba & slender hawksbeard & $\mathrm{s}$ & 662 & 0 \\
\hline 2 & 1572 & Crepis atribarba & slender hawksbeard & $\mathrm{s}$ & 691 & 0 \\
\hline 2 & 1573 & Crepis atribarba & slender hawksbeard & S & 706 & 0 \\
\hline 2 & 1574 & Crepis atribarba & slender hawksbeard & $\mathrm{s}$ & 647 & 0 \\
\hline 2 & 1575 & Crepis atribarba & slender hawksbeard & $\mathrm{s}$ & 721 & 0 \\
\hline 2 & 1576 & Crepis atribarba & slender hawksbeard & $\mathrm{s}$ & 662 & 0 \\
\hline 2 & 1577 & Crepis atribarba & slender hawksbeard & s & 735 & 0 \\
\hline 2 & 1578 & Crepis atribarba & slender hawksbeard & s & 647 & 0 \\
\hline
\end{tabular}


Table C.4. (contd)

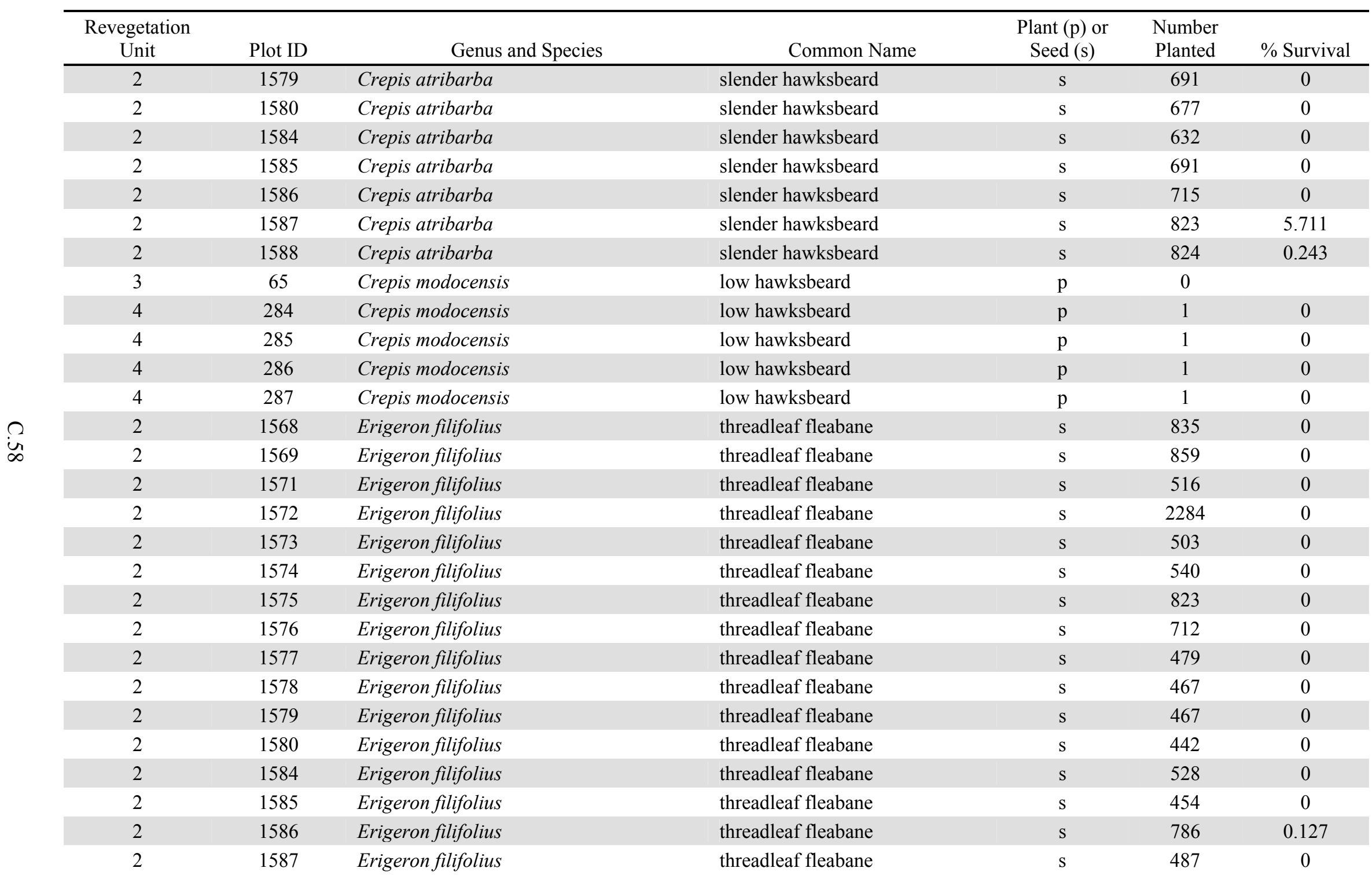


Table C.4. (contd)

\begin{tabular}{|c|c|c|c|c|c|c|}
\hline $\begin{array}{c}\text { Revegetation } \\
\text { Unit }\end{array}$ & Plot ID & Genus and Species & Common Name & $\begin{array}{l}\text { Plant (p) or } \\
\text { Seed (s) }\end{array}$ & $\begin{array}{l}\text { Number } \\
\text { Planted }\end{array}$ & $\%$ Survival \\
\hline 2 & 1588 & Erigeron filifolius & threadleaf fleabane & $\mathrm{S}$ & 540 & 0 \\
\hline 3 & 65 & Erigeron linearis & desert yellowdaisy & $\mathrm{p}$ & 43 & 48.84 \\
\hline 4 & 64 & Erigeron linearis & desert yellowdaisy & $\mathrm{p}$ & 43 & 72.09 \\
\hline 4 & 260 & Erigeron linearis & desert yellowdaisy & $\mathrm{s}$ & 50 & 0 \\
\hline 4 & 261 & Erigeron linearis & desert yellowdaisy & $\mathrm{s}$ & 50 & 0 \\
\hline 4 & 262 & Erigeron linearis & desert yellowdaisy & $\mathrm{s}$ & 50 & 0 \\
\hline 4 & 263 & Erigeron linearis & desert yellowdaisy & $\mathrm{s}$ & 50 & 0 \\
\hline 4 & 264 & Erigeron linearis & desert yellowdaisy & s & 50 & 0 \\
\hline 4 & 265 & Erigeron linearis & desert yellowdaisy & $\mathrm{s}$ & 50 & 0 \\
\hline 4 & 269 & Erigeron linearis & desert yellowdaisy & $\mathrm{S}$ & 50 & 0 \\
\hline 4 & 270 & Erigeron linearis & desert yellowdaisy & $\mathrm{s}$ & 50 & 0 \\
\hline 4 & 272 & Erigeron linearis & desert yellowdaisy & $\mathrm{S}$ & 50 & 0 \\
\hline 4 & 273 & Erigeron linearis & desert yellowdaisy & $\mathrm{s}$ & 50 & 0 \\
\hline 4 & 274 & Erigeron linearis & desert yellowdaisy & $\mathrm{s}$ & 50 & 0 \\
\hline 4 & 275 & Erigeron linearis & desert yellowdaisy & $\mathrm{s}$ & 50 & 0 \\
\hline 4 & 276 & Erigeron linearis & desert yellowdaisy & $\mathrm{s}$ & 50 & 0 \\
\hline 4 & 284 & Erigeron linearis & desert yellowdaisy & $\mathrm{p}$ & 43 & 90.7 \\
\hline 4 & 285 & Erigeron linearis & desert yellowdaisy & $\mathrm{p}$ & 43 & 79.07 \\
\hline 4 & 286 & Erigeron linearis & desert yellowdaisy & $\mathrm{p}$ & 43 & 72.09 \\
\hline 4 & 287 & Erigeron linearis & desert yellowdaisy & $\mathrm{p}$ & 43 & 86.05 \\
\hline 5 & 266 & Erigeron linearis & desert yellowdaisy & $\mathrm{s}$ & 50 & 0 \\
\hline 5 & 267 & Erigeron linearis & desert yellowdaisy & $\mathrm{s}$ & 50 & 0 \\
\hline 5 & 268 & Erigeron linearis & desert yellowdaisy & $\mathrm{s}$ & 50 & 0 \\
\hline 5 & 1798 & Erigeron linearis & desert yellowdaisy & $\mathrm{p}$ & 43 & 51.16 \\
\hline 6 & 66 & Erigeron linearis & desert yellowdaisy & $\mathrm{p}$ & 43 & 72.09 \\
\hline 6 & 277 & Erigeron linearis & desert yellowdaisy & $\mathrm{S}$ & 50 & 0 \\
\hline 6 & 278 & Erigeron linearis & desert yellowdaisy & $\mathrm{s}$ & 50 & 0 \\
\hline 6 & 279 & Erigeron linearis & desert yellowdaisy & $\mathrm{S}$ & 50 & 0 \\
\hline
\end{tabular}


Table C.4. (contd)

\begin{tabular}{|c|c|c|c|c|c|c|}
\hline $\begin{array}{l}\text { Revegetation } \\
\text { Unit }\end{array}$ & Plot ID & Genus and Species & Common Name & $\begin{array}{l}\text { Plant (p) or } \\
\text { Seed (s) }\end{array}$ & $\begin{array}{l}\text { Number } \\
\text { Planted }\end{array}$ & $\%$ Survival \\
\hline 6 & 1590 & Erigeron linearis & desert yellowdaisy & $\mathrm{p}$ & 44 & 68.18 \\
\hline 6 & 1591 & Erigeron linearis & desert yellowdaisy & $\mathrm{p}$ & 43 & 65.12 \\
\hline 2 & 280 & Erigeron piperianus & Piper's daisy & $\mathrm{p}$ & 39 & 87.18 \\
\hline 2 & 281 & Erigeron piperianus & Piper's daisy & $\mathrm{p}$ & 39 & 82.05 \\
\hline 2 & 282 & Erigeron piperianus & Piper's daisy & $\mathrm{p}$ & 39 & 89.74 \\
\hline 2 & 283 & Erigeron piperianus & Piper's daisy & $\mathrm{p}$ & 39 & 100 \\
\hline 2 & 1568 & Erigeron piperianus & Piper's daisy & $\mathrm{s}$ & 233 & 0 \\
\hline 2 & 1569 & Erigeron piperianus & Piper's daisy & $\mathrm{s}$ & 233 & 0 \\
\hline 2 & 1571 & Erigeron piperianus & Piper's daisy & $\mathrm{s}$ & 247 & 0 \\
\hline 2 & 1572 & Erigeron piperianus & Piper's daisy & $\mathrm{s}$ & 246 & 0 \\
\hline 2 & 1573 & Erigeron piperianus & Piper's daisy & $\mathrm{s}$ & 289 & 1.038 \\
\hline 2 & 1574 & Erigeron piperianus & Piper's daisy & $\mathrm{s}$ & 251 & 0 \\
\hline 2 & 1575 & Erigeron piperianus & Piper's daisy & $\mathrm{s}$ & 215 & 0 \\
\hline 2 & 1576 & Erigeron piperianus & Piper's daisy & $\mathrm{s}$ & 34 & 0 \\
\hline 2 & 1577 & Erigeron piperianus & Piper's daisy & $\mathrm{s}$ & 293 & 0 \\
\hline 2 & 1578 & Erigeron piperianus & Piper's daisy & $\mathrm{s}$ & 213 & 0 \\
\hline 2 & 1579 & Erigeron piperianus & Piper's daisy & $\mathrm{s}$ & 129 & 0 \\
\hline 2 & 1580 & Erigeron piperianus & Piper's daisy & $\mathrm{s}$ & 191 & 0 \\
\hline 2 & 1584 & Erigeron piperianus & Piper's daisy & $\mathrm{s}$ & 175 & 0 \\
\hline 2 & 1585 & Erigeron piperianus & Piper's daisy & $\mathrm{s}$ & 215 & 0 \\
\hline 2 & 1586 & Erigeron piperianus & Piper's daisy & $\mathrm{s}$ & 177 & 0 \\
\hline 2 & 1587 & Erigeron piperianus & Piper's daisy & $\mathrm{s}$ & 292 & 0 \\
\hline 2 & 1588 & Erigeron piperianus & Piper's daisy & $\mathrm{s}$ & 241 & 0.415 \\
\hline 2 & 1697 & Erigeron piperianus & Piper's daisy & $\mathrm{p}$ & 39 & 105.1 \\
\hline 2 & 1697 & Erigeron piperianus & Piper's daisy & $\mathrm{p}$ & 39 & 92.31 \\
\hline 2 & 1698 & Erigeron piperianus & Piper's daisy & $\mathrm{p}$ & 39 & 89.74 \\
\hline 3 & 65 & Erigeron poliospermus & cushion fleabane & $\mathrm{p}$ & 3 & 100 \\
\hline 4 & 64 & Erigeron poliospermus & cushion fleabane & $\mathrm{p}$ & 3 & 0 \\
\hline
\end{tabular}


Table C.4. (contd)

\begin{tabular}{|c|c|c|c|c|c|c|}
\hline $\begin{array}{l}\text { Revegetation } \\
\text { Unit }\end{array}$ & Plot ID & Genus and Species & Common Name & $\begin{array}{l}\text { Plant }(\mathrm{p}) \text { or } \\
\text { Seed }(\mathrm{s})\end{array}$ & $\begin{array}{l}\text { Number } \\
\text { Planted }\end{array}$ & $\%$ Survival \\
\hline 4 & 260 & Erigeron poliospermus & cushion fleabane & $\mathrm{S}$ & 50 & 0 \\
\hline 4 & 261 & Erigeron poliospermus & cushion fleabane & $\mathrm{s}$ & 50 & 0 \\
\hline 4 & 262 & Erigeron poliospermus & cushion fleabane & s & 50 & 0 \\
\hline 4 & 263 & Erigeron poliospermus & cushion fleabane & s & 50 & 0 \\
\hline 4 & 264 & Erigeron poliospermus & cushion fleabane & $\mathrm{s}$ & 50 & 0 \\
\hline 4 & 265 & Erigeron poliospermus & cushion fleabane & $\mathrm{s}$ & 50 & 0 \\
\hline 4 & 269 & Erigeron poliospermus & cushion fleabane & $\mathrm{s}$ & 50 & 0 \\
\hline 4 & 270 & Erigeron poliospermus & cushion fleabane & $\mathrm{s}$ & 50 & 0 \\
\hline 4 & 272 & Erigeron poliospermus & cushion fleabane & $\mathrm{s}$ & 50 & 0 \\
\hline 4 & 273 & Erigeron poliospermus & cushion fleabane & $\mathrm{s}$ & 50 & 0 \\
\hline 4 & 274 & Erigeron poliospermus & cushion fleabane & s & 50 & 0 \\
\hline 4 & 275 & Erigeron poliospermus & cushion fleabane & $\mathrm{s}$ & 50 & 0 \\
\hline 4 & 276 & Erigeron poliospermus & cushion fleabane & $\mathrm{s}$ & 50 & 0 \\
\hline 4 & 284 & Erigeron poliospermus & cushion fleabane & $\mathrm{p}$ & 3 & 100 \\
\hline 4 & 285 & Erigeron poliospermus & cushion fleabane & $\mathrm{p}$ & 3 & 100 \\
\hline 4 & 286 & Erigeron poliospermus & cushion fleabane & $\mathrm{p}$ & 3 & 66.67 \\
\hline 4 & 287 & Erigeron poliospermus & cushion fleabane & $\mathrm{p}$ & 3 & 33.33 \\
\hline 5 & 266 & Erigeron poliospermus & cushion fleabane & s & 50 & 0 \\
\hline 5 & 267 & Erigeron poliospermus & cushion fleabane & $\mathrm{s}$ & 50 & 0 \\
\hline 5 & 268 & Erigeron poliospermus & cushion fleabane & $\mathrm{s}$ & 50 & 0 \\
\hline 5 & 1798 & Erigeron poliospermus & cushion fleabane & $\mathrm{p}$ & 3 & 100 \\
\hline 6 & 66 & Erigeron poliospermus & cushion fleabane & $\mathrm{p}$ & 3 & 0 \\
\hline 6 & 277 & Erigeron poliospermus & cushion fleabane & $\mathrm{s}$ & 50 & 0 \\
\hline 6 & 279 & Erigeron poliospermus & cushion fleabane & s & 50 & 0 \\
\hline 6 & 1590 & Erigeron poliospermus & cushion fleabane & $\mathrm{p}$ & 3 & 100 \\
\hline 6 & 1591 & Erigeron poliospermus & cushion fleabane & $\mathrm{p}$ & 6 & 83.33 \\
\hline 3 & 65 & Eriogonum sphaerocephalum & rock buckwheat & $\mathrm{p}$ & 4 & 25 \\
\hline 3 & 1589 & Eriogonum sphaerocephalum & rock buckwheat & $\mathrm{s}$ & 76 & 0 \\
\hline
\end{tabular}


Table C.4. (contd)

\begin{tabular}{|c|c|c|c|c|c|c|}
\hline $\begin{array}{c}\text { Revegetation } \\
\text { Unit }\end{array}$ & Plot ID & Genus and Species & Common Name & $\begin{array}{l}\text { Plant (p) or } \\
\text { Seed (s) }\end{array}$ & $\begin{array}{l}\text { Number } \\
\text { Planted }\end{array}$ & $\%$ Survival \\
\hline 4 & 64 & Eriogonum sphaerocephalum & rock buckwheat & $p$ & 4 & 25 \\
\hline 4 & 260 & Eriogonum sphaerocephalum & rock buckwheat & $\mathrm{s}$ & 50 & 0 \\
\hline 4 & 261 & Eriogonum sphaerocephalum & rock buckwheat & $\mathrm{s}$ & 50 & 0 \\
\hline 4 & 262 & Eriogonum sphaerocephalum & rock buckwheat & $\mathrm{s}$ & 50 & 0 \\
\hline 4 & 263 & Eriogonum sphaerocephalum & rock buckwheat & $\mathrm{s}$ & 50 & 0 \\
\hline 4 & 264 & Eriogonum sphaerocephalum & rock buckwheat & $\mathrm{s}$ & 50 & 0 \\
\hline 4 & 265 & Eriogonum sphaerocephalum & rock buckwheat & $\mathrm{s}$ & 50 & 0 \\
\hline 4 & 269 & Eriogonum sphaerocephalum & rock buckwheat & $\mathrm{s}$ & 50 & 0 \\
\hline 4 & 270 & Eriogonum sphaerocephalum & rock buckwheat & $\mathrm{s}$ & 50 & 0 \\
\hline 4 & 272 & Eriogonum sphaerocephalum & rock buckwheat & $\mathrm{s}$ & 50 & 0 \\
\hline 4 & 273 & Eriogonum sphaerocephalum & rock buckwheat & $\mathrm{s}$ & 50 & 0 \\
\hline 4 & 274 & Eriogonum sphaerocephalum & rock buckwheat & $\mathrm{s}$ & 50 & 0 \\
\hline 4 & 275 & Eriogonum sphaerocephalum & rock buckwheat & $\mathrm{s}$ & 50 & 0 \\
\hline 4 & 276 & Eriogonum sphaerocephalum & rock buckwheat & $\mathrm{s}$ & 50 & 0 \\
\hline 4 & 284 & Eriogonum sphaerocephalum & rock buckwheat & $\mathrm{p}$ & 3 & 0 \\
\hline 4 & 285 & Eriogonum sphaerocephalum & rock buckwheat & $\mathrm{p}$ & 3 & 66.67 \\
\hline 4 & 286 & Eriogonum sphaerocephalum & rock buckwheat & $\mathrm{p}$ & 3 & 66.67 \\
\hline 4 & 287 & Eriogonum sphaerocephalum & rock buckwheat & $\mathrm{p}$ & 3 & 100 \\
\hline 5 & 266 & Eriogonum sphaerocephalum & rock buckwheat & $\mathrm{s}$ & 50 & 0 \\
\hline 5 & 267 & Eriogonum sphaerocephalum & rock buckwheat & $\mathrm{s}$ & 50 & 0 \\
\hline 5 & 268 & Eriogonum sphaerocephalum & rock buckwheat & $\mathrm{s}$ & 50 & 0 \\
\hline 6 & 66 & Eriogonum sphaerocephalum & rock buckwheat & $\mathrm{p}$ & 4 & 50 \\
\hline 6 & 277 & Eriogonum sphaerocephalum & rock buckwheat & $\mathrm{s}$ & 50 & 0 \\
\hline 6 & 278 & Eriogonum sphaerocephalum & rock buckwheat & $\mathrm{s}$ & 50 & 0 \\
\hline 6 & 279 & Eriogonum sphaerocephalum & rock buckwheat & $\mathrm{s}$ & 50 & 0 \\
\hline 6 & 1590 & Eriogonum sphaerocephalum & rock buckwheat & $\mathrm{p}$ & 4 & 100 \\
\hline 6 & 1591 & Eriogonum sphaerocephalum & rock buckwheat & $\mathrm{p}$ & 6 & 50 \\
\hline 3 & 65 & Eriogonum thymoides & thymeleaf buckwheat & $\mathrm{p}$ & 3 & 66.67 \\
\hline
\end{tabular}


Table C.4. (contd)

\begin{tabular}{|c|c|c|c|c|c|c|}
\hline $\begin{array}{c}\text { Revegetation } \\
\text { Unit }\end{array}$ & Plot ID & Genus and Species & Common Name & $\begin{array}{l}\text { Plant (p) or } \\
\text { Seed (s) }\end{array}$ & $\begin{array}{l}\text { Number } \\
\text { Planted }\end{array}$ & $\%$ Survival \\
\hline 4 & 64 & Eriogonum thymoides & thymeleaf buckwheat & $\mathrm{p}$ & 3 & 66.67 \\
\hline 4 & 284 & Eriogonum thymoides & thymeleaf buckwheat & $\mathrm{p}$ & 2 & 50 \\
\hline 4 & 285 & Eriogonum thymoides & thymeleaf buckwheat & $\mathrm{p}$ & 3 & 33.33 \\
\hline 4 & 286 & Eriogonum thymoides & thymeleaf buckwheat & $\mathrm{p}$ & 3 & 100 \\
\hline 4 & 287 & Eriogonum thymoides & thymeleaf buckwheat & $\mathrm{p}$ & 3 & 100 \\
\hline 6 & 66 & Eriogonum thymoides & thymeleaf buckwheat & $\mathrm{p}$ & 2 & 50 \\
\hline 6 & 1590 & Eriogonum thymoides & thymeleaf buckwheat & $\mathrm{p}$ & 2 & 50 \\
\hline 6 & 1591 & Eriogonum thymoides & thymeleaf buckwheat & $\mathrm{p}$ & 2 & 0 \\
\hline 4 & 260 & Eriophyllum lanatum & woolly sunflower & $\mathrm{s}$ & 50 & 0 \\
\hline 4 & 261 & Eriophyllum lanatum & woolly sunflower & $\mathrm{s}$ & 50 & 0 \\
\hline 4 & 262 & Eriophyllum lanatum & woolly sunflower & $\mathrm{s}$ & 50 & 0 \\
\hline 4 & 263 & Eriophyllum lanatum & woolly sunflower & $\mathrm{s}$ & 50 & 0 \\
\hline 4 & 264 & Eriophyllum lanatum & woolly sunflower & $\mathrm{s}$ & 50 & 0 \\
\hline 4 & 265 & Eriophyllum lanatum & woolly sunflower & $\mathrm{s}$ & 50 & 0 \\
\hline 4 & 269 & Eriophyllum lanatum & woolly sunflower & $\mathrm{s}$ & 50 & 0 \\
\hline 4 & 270 & Eriophyllum lanatum & woolly sunflower & $\mathrm{s}$ & 50 & 0 \\
\hline 4 & 272 & Eriophyllum lanatum & woolly sunflower & $\mathrm{s}$ & 50 & 0 \\
\hline 4 & 273 & Eriophyllum lanatum & woolly sunflower & $\mathrm{s}$ & 50 & 0 \\
\hline 4 & 274 & Eriophyllum lanatum & woolly sunflower & $\mathrm{s}$ & 50 & 0 \\
\hline 4 & 275 & Eriophyllum lanatum & woolly sunflower & $\mathrm{s}$ & 50 & 0 \\
\hline 4 & 276 & Eriophyllum lanatum & woolly sunflower & $\mathrm{s}$ & 50 & 0 \\
\hline 5 & 266 & Eriophyllum lanatum & woolly sunflower & $\mathrm{s}$ & 50 & 0 \\
\hline 5 & 267 & Eriophyllum lanatum & woolly sunflower & $\mathrm{s}$ & 50 & 0 \\
\hline 5 & 268 & Eriophyllum lanatum & woolly sunflower & $\mathrm{s}$ & 50 & 0 \\
\hline 6 & 277 & Eriophyllum lanatum & woolly sunflower & $\mathrm{s}$ & 50 & 0 \\
\hline 6 & 278 & Eriophyllum lanatum & woolly sunflower & $\mathrm{s}$ & 50 & 0 \\
\hline 6 & 279 & Eriophyllum lanatum & woolly sunflower & $\mathrm{s}$ & 50 & 0 \\
\hline 1 & 1581 & Grayia spinosa & spiny hopsage & s & 1638 & 0 \\
\hline
\end{tabular}


Table C.4. (contd)

\begin{tabular}{|c|c|c|c|c|c|c|}
\hline $\begin{array}{l}\text { Revegetation } \\
\text { Unit }\end{array}$ & Plot ID & Genus and Species & Common Name & $\begin{array}{l}\text { Plant (p) or } \\
\text { Seed (s) }\end{array}$ & $\begin{array}{l}\text { Number } \\
\text { Planted }\end{array}$ & $\%$ Survival \\
\hline 1 & 1582 & Grayia spinosa & spiny hopsage & $\mathrm{s}$ & 1646 & 0 \\
\hline 1 & 1583 & Grayia spinosa & spiny hopsage & $\mathrm{s}$ & 1650 & 0 \\
\hline 1 & Circle & Grayia spinosa & spiny hopsage & $\mathrm{p}$ & 150 & 9.333 \\
\hline 1 & Road & Grayia spinosa & spiny hopsage & $\mathrm{p}$ & 150 & 25.33 \\
\hline 2 & 1568 & Helianthus cusickii & Cusicks sunflower & $\mathrm{s}$ & 587 & 0.511 \\
\hline 2 & 1584 & Helianthus cusickii & Cusicks sunflower & $\mathrm{s}$ & 443 & 0 \\
\hline 2 & 1586 & Helianthus cusickii & Cusicks sunflower & $\mathrm{s}$ & 435 & 1.149 \\
\hline 4 & 260 & Lomatium grayi & Gray's desertparsley & s & 50 & 0 \\
\hline 4 & 261 & Lomatium grayi & Gray's desertparsley & $\mathrm{s}$ & 50 & 0 \\
\hline 4 & 262 & Lomatium grayi & Gray's desertparsley & s & 50 & 0 \\
\hline 4 & 263 & Lomatium grayi & Gray's desertparsley & $\mathrm{s}$ & 50 & 0 \\
\hline 4 & 264 & Lomatium grayi & Gray's desertparsley & $\mathrm{s}$ & 50 & 0 \\
\hline 4 & 265 & Lomatium grayi & Gray's desertparsley & $\mathrm{s}$ & 50 & 0 \\
\hline 4 & 269 & Lomatium grayi & Gray's desertparsley & $\mathrm{s}$ & 50 & 0 \\
\hline 4 & 270 & Lomatium grayi & Gray's desertparsley & $\mathrm{s}$ & 50 & 0 \\
\hline 4 & 272 & Lomatium grayi & Gray's desertparsley & s & 50 & 0 \\
\hline 4 & 273 & Lomatium grayi & Gray's desertparsley & $\mathrm{s}$ & 50 & 0 \\
\hline 4 & 274 & Lomatium grayi & Gray's desertparsley & $\mathrm{s}$ & 50 & 0 \\
\hline 4 & 275 & Lomatium grayi & Gray's desertparsley & s & 50 & 0 \\
\hline 4 & 276 & Lomatium grayi & Gray's desertparsley & $\mathrm{s}$ & 50 & 0 \\
\hline 5 & 266 & Lomatium grayi & Gray's desertparsley & $\mathrm{s}$ & 50 & 0 \\
\hline 5 & 267 & Lomatium grayi & Gray's desertparsley & $\mathrm{s}$ & 50 & 0 \\
\hline 5 & 268 & Lomatium grayi & Gray's desertparsley & S & 50 & 0 \\
\hline 6 & 277 & Lomatium grayi & Gray's desertparsley & $\mathrm{s}$ & 50 & 0 \\
\hline 6 & 278 & Lomatium grayi & Gray's desertparsley & $\mathrm{s}$ & 50 & 0 \\
\hline 6 & 279 & Lomatium grayi & Gray's desertparsley & s & 50 & 0 \\
\hline 3 & 1589 & Lomatium macrocarpum & bigseed desertparsley & s & 400 & 0 \\
\hline 4 & 269 & Lomatium triternatum & nineleaf desertparsley & s & 50 & 0 \\
\hline
\end{tabular}


Table C.4. (contd)

\begin{tabular}{|c|c|c|c|c|c|c|}
\hline $\begin{array}{l}\text { Revegetation } \\
\text { Unit }\end{array}$ & Plot ID & Genus and Species & Common Name & $\begin{array}{l}\text { Plant (p) or } \\
\text { Seed (s) }\end{array}$ & $\begin{array}{l}\text { Number } \\
\text { Planted }\end{array}$ & $\%$ Survival \\
\hline 4 & 272 & Lomatium triternatum & nineleaf desertparsley & $\mathrm{s}$ & 50 & 0 \\
\hline 5 & 268 & Lomatium triternatum & nineleaf desertparsley & $\mathrm{s}$ & 50 & 0 \\
\hline 2 & 280 & Lupinus sericeus & silky lupine & $\mathrm{p}$ & 6 & 83.33 \\
\hline 2 & 281 & Lupinus sericeus & silky lupine & $\mathrm{p}$ & 6 & 100 \\
\hline 2 & 1568 & Lupinus sericeus & silky lupine & $\mathrm{s}$ & 92 & 2.174 \\
\hline 2 & 1569 & Lupinus sericeus & silky lupine & $\mathrm{s}$ & 77 & 1.299 \\
\hline 2 & 1571 & Lupinus sericeus & silky lupine & $\mathrm{s}$ & 85 & 5.882 \\
\hline 2 & 1572 & Lupinus sericeus & silky lupine & $\mathrm{s}$ & 81 & 0 \\
\hline 2 & 1573 & Lupinus sericeus & silky lupine & $\mathrm{s}$ & 87 & 6.897 \\
\hline 2 & 1578 & Lupinus sericeus & silky lupine & $\mathrm{s}$ & 78 & 1.282 \\
\hline 2 & 1579 & Lupinus sericeus & silky lupine & $\mathrm{s}$ & 82 & 2.439 \\
\hline 2 & 1580 & Lupinus sericeus & silky lupine & $\mathrm{s}$ & 82 & 2.439 \\
\hline 2 & 1584 & Lupinus sericeus & silky lupine & $\mathrm{s}$ & 79 & 0 \\
\hline 2 & 1585 & Lupinus sericeus & silky lupine & $\mathrm{s}$ & 83 & 9.639 \\
\hline 2 & 1586 & Lupinus sericeus & silky lupine & $\mathrm{s}$ & 68 & 0 \\
\hline 2 & 1587 & Lupinus sericeus & silky lupine & $\mathrm{s}$ & 87 & 2.299 \\
\hline 2 & 1588 & Lupinus sericeus & silky lupine & $\mathrm{s}$ & 76 & 2.632 \\
\hline 2 & 1697 & Lupinus sericeus & silky lupine & $\mathrm{p}$ & 6 & 83.33 \\
\hline 2 & 1697 & Lupinus sericeus & silky lupine & $\mathrm{p}$ & 6 & 83.33 \\
\hline 2 & 1698 & Lupinus sericeus & silky lupine & $\mathrm{p}$ & 6 & 100 \\
\hline
\end{tabular}


Table C.4. (contd)

\begin{tabular}{|c|c|c|c|c|c|c|}
\hline $\begin{array}{l}\text { Revegetation } \\
\text { Unit }\end{array}$ & Plot ID & Genus and Species & Common Name & $\begin{array}{l}\text { Plant (p) or } \\
\text { Seed (s) }\end{array}$ & $\begin{array}{l}\text { Number } \\
\text { Planted }\end{array}$ & $\%$ Survival \\
\hline 1 & 1581 & Machaeranthera canescens & hoary aster & $\mathrm{s}$ & 2264 & 0 \\
\hline 1 & 1583 & Machaeranthera canescens & hoary aster & $\mathrm{s}$ & 4135 & 0.048 \\
\hline 2 & 1568 & Machaeranthera canescens & hoary aster & $\mathrm{s}$ & 3192 & 0.439 \\
\hline 2 & 1569 & Machaeranthera canescens & hoary aster & $\mathrm{s}$ & 2838 & 3.242 \\
\hline 2 & 1573 & Machaeranthera canescens & hoary aster & $\mathrm{s}$ & 2901 & 5.619 \\
\hline 2 & 1574 & Machaeranthera canescens & hoary aster & $\mathrm{s}$ & 4096 & 3.833 \\
\hline 2 & 1575 & Machaeranthera canescens & hoary aster & $\mathrm{s}$ & 2767 & 1.662 \\
\hline 2 & 1576 & Machaeranthera canescens & hoary aster & $\mathrm{s}$ & 4277 & 3.04 \\
\hline 2 & 1577 & Machaeranthera canescens & hoary aster & $\mathrm{s}$ & 2728 & 1.65 \\
\hline 2 & 1585 & Machaeranthera canescens & hoary aster & $\mathrm{s}$ & 3451 & 5.911 \\
\hline 2 & 1586 & Machaeranthera canescens & hoary aster & $\mathrm{s}$ & 2610 & 7.088 \\
\hline 2 & 1587 & Machaeranthera canescens & hoary aster & $\mathrm{s}$ & 2311 & 8.351 \\
\hline 2 & 1588 & Machaeranthera canescens & hoary aster & $\mathrm{s}$ & 2508 & 9.37 \\
\hline 3 & 65 & Penstemon speciosus & showy beardtongue & $\mathrm{p}$ & 1 & 100 \\
\hline 4 & 64 & Penstemon speciosus & showy beardtongue & $\mathrm{p}$ & 1 & 0 \\
\hline 4 & 260 & Penstemon speciosus & showy beardtongue & $\mathrm{s}$ & 50 & 0 \\
\hline 4 & 261 & Penstemon speciosus & showy beardtongue & $\mathrm{s}$ & 50 & 0 \\
\hline 4 & 262 & Penstemon speciosus & showy beardtongue & $\mathrm{s}$ & 50 & 0 \\
\hline 4 & 263 & Penstemon speciosus & showy beardtongue & $\mathrm{s}$ & 50 & 0 \\
\hline 4 & 264 & Penstemon speciosus & showy beardtongue & $\mathrm{s}$ & 50 & 0 \\
\hline
\end{tabular}


Table C.4. (contd)

\begin{tabular}{|c|c|c|c|c|c|c|}
\hline $\begin{array}{c}\text { Revegetation } \\
\text { Unit }\end{array}$ & Plot ID & Genus and Species & Common Name & $\begin{array}{l}\text { Plant (p) or } \\
\text { Seed (s) }\end{array}$ & $\begin{array}{l}\text { Number } \\
\text { Planted }\end{array}$ & $\%$ Survival \\
\hline 4 & 265 & Penstemon speciosus & showy beardtongue & $\mathrm{s}$ & 50 & 0 \\
\hline 4 & 284 & Penstemon speciosus & showy beardtongue & $\mathrm{p}$ & 1 & 100 \\
\hline 4 & 285 & Penstemon speciosus & showy beardtongue & $\mathrm{p}$ & 1 & 100 \\
\hline 4 & 286 & Penstemon speciosus & showy beardtongue & $\mathrm{p}$ & 1 & 100 \\
\hline 4 & 287 & Penstemon speciosus & showy beardtongue & $\mathrm{p}$ & 2 & 100 \\
\hline 5 & 266 & Penstemon speciosus & showy beardtongue & $\mathrm{s}$ & 50 & 0 \\
\hline 5 & 267 & Penstemon speciosus & showy beardtongue & $\mathrm{s}$ & 50 & 0 \\
\hline 5 & 1798 & Penstemon speciosus & showy beardtongue & $\mathrm{p}$ & 1 & 0 \\
\hline 6 & 66 & Penstemon speciosus & showy beardtongue & $\mathrm{p}$ & 1 & 100 \\
\hline 6 & 1590 & Penstemon speciosus & showy beardtongue & $\mathrm{p}$ & 1 & 0 \\
\hline 6 & 1591 & Penstemon speciosus & showy beardtongue & $\mathrm{p}$ & 2 & 100 \\
\hline 3 & 65 & Phlox hoodii & Hood's phlox & $\mathrm{p}$ & 1 & 100 \\
\hline 4 & 64 & Phlox hoodii & Hood's phlox & $\mathrm{p}$ & 1 & 0 \\
\hline 4 & 284 & Phlox hoodii & Hood's phlox & $\mathrm{p}$ & 1 & 100 \\
\hline 4 & 285 & Phlox hoodii & Hood's phlox & $\mathrm{p}$ & 1 & 100 \\
\hline 4 & 286 & Phlox hoodii & Hood's phlox & $\mathrm{p}$ & 1 & 100 \\
\hline 4 & 287 & Phlox hoodii & Hood's phlox & $\mathrm{p}$ & 1 & 100 \\
\hline 5 & 1798 & Phlox hoodii & Hood's phlox & $\mathrm{p}$ & 1 & 100 \\
\hline 6 & 66 & Phlox hoodii & Hood's phlox & $\mathrm{p}$ & 1 & 100 \\
\hline 6 & 1590 & Phlox hoodii & Hood's phlox & $\mathrm{p}$ & 2 & 100 \\
\hline 6 & 1591 & Phlox hoodii & Hood's phlox & $\mathrm{p}$ & 3 & 0 \\
\hline 2 & 280 & Phlox longifolia & longleaf phlox & $\mathrm{p}$ & 16 & 75 \\
\hline 2 & 281 & Phlox longifolia & longleaf phlox & $\mathrm{p}$ & 16 & 56.25 \\
\hline 2 & 282 & Phlox longifolia & longleaf phlox & $\mathrm{p}$ & 16 & 75 \\
\hline 2 & 283 & Phlox longifolia & longleaf phlox & $\mathrm{p}$ & 16 & 75 \\
\hline 2 & 1697 & Phlox longifolia & longleaf phlox & $\mathrm{p}$ & 16 & 75 \\
\hline 2 & 1697 & Phlox longifolia & longleaf phlox & $\mathrm{p}$ & 16 & 81.25 \\
\hline 2 & 1698 & Phlox longifolia & longleaf phlox & $\mathrm{p}$ & 16 & 93.75 \\
\hline
\end{tabular}


Table C.4. (contd)

\begin{tabular}{|c|c|c|c|c|c|c|}
\hline $\begin{array}{l}\text { Revegetation } \\
\text { Unit }\end{array}$ & Plot ID & Genus and Species & Common Name & $\begin{array}{l}\text { Plant }(\mathrm{p}) \text { or } \\
\text { Seed }(\mathrm{s})\end{array}$ & $\begin{array}{l}\text { Number } \\
\text { Planted }\end{array}$ & $\%$ Survival \\
\hline 3 & 65 & Phlox speciosa & showy phlox & $\mathrm{p}$ & 15 & 0 \\
\hline 4 & 64 & Phlox speciosa & showy phlox & $\mathrm{p}$ & 15 & 6.667 \\
\hline 4 & 260 & Phlox speciosa & showy phlox & $\mathrm{s}$ & 50 & 0 \\
\hline 4 & 261 & Phlox speciosa & showy phlox & $\mathrm{s}$ & 50 & 0 \\
\hline 4 & 262 & Phlox speciosa & showy phlox & $\mathrm{s}$ & 50 & 0 \\
\hline 4 & 263 & Phlox speciosa & showy phlox & $\mathrm{s}$ & 50 & 0 \\
\hline 4 & 264 & Phlox speciosa & showy phlox & s & 50 & 0 \\
\hline 4 & 265 & Phlox speciosa & showy phlox & $\mathrm{s}$ & 50 & 0 \\
\hline 4 & 285 & Phlox speciosa & showy phlox & $\mathrm{p}$ & 15 & 0 \\
\hline 4 & 286 & Phlox speciosa & showy phlox & $\mathrm{p}$ & 15 & 13.33 \\
\hline 4 & 287 & Phlox speciosa & showy phlox & $\mathrm{p}$ & 15 & 6.667 \\
\hline 5 & 266 & Phlox speciosa & showy phlox & $\mathrm{s}$ & 50 & 0 \\
\hline 5 & 267 & Phlox speciosa & showy phlox & $\mathrm{s}$ & 50 & 0 \\
\hline 5 & 268 & Phlox speciosa & showy phlox & $\mathrm{s}$ & 50 & 0 \\
\hline 5 & 1798 & Phlox speciosa & showy phlox & $\mathrm{p}$ & 15 & 6.667 \\
\hline 6 & 66 & Phlox speciosa & showy phlox & $\mathrm{p}$ & 15 & 6.667 \\
\hline 6 & 1590 & Phlox speciosa & showy phlox & $\mathrm{p}$ & 15 & 13.33 \\
\hline 6 & 1591 & Phlox speciosa & showy phlox & $\mathrm{p}$ & 8 & 0 \\
\hline 3 & 65 & Salvia dorrii & grayball sage & $\mathrm{p}$ & 5 & 80 \\
\hline 4 & 64 & Salvia dorrii & grayball sage & $\mathrm{p}$ & 5 & 100 \\
\hline 4 & 260 & Salvia dorrii & grayball sage & $\mathrm{s}$ & 50 & 0 \\
\hline 4 & 261 & Salvia dorrii & grayball sage & $\mathrm{s}$ & 50 & 0 \\
\hline 4 & 262 & Salvia dorrii & grayball sage & $\mathrm{s}$ & 50 & 0 \\
\hline 4 & 263 & Salvia dorrii & grayball sage & $\mathrm{s}$ & 50 & 0 \\
\hline 4 & 264 & Salvia dorrii & grayball sage & $\mathrm{s}$ & 50 & 0 \\
\hline 4 & 265 & Salvia dorrii & grayball sage & $\mathrm{s}$ & 50 & 0 \\
\hline 4 & 284 & Salvia dorrii & grayball sage & $\mathrm{p}$ & 5 & 100 \\
\hline 4 & 285 & Salvia dorrii & grayball sage & $\mathrm{p}$ & 5 & 80 \\
\hline
\end{tabular}


Table C.4. (contd)

\begin{tabular}{|c|c|c|c|c|c|c|}
\hline $\begin{array}{l}\text { Revegetation } \\
\text { Unit }\end{array}$ & Plot ID & Genus and Species & Common Name & $\begin{array}{l}\text { Plant }(\mathrm{p}) \text { or } \\
\text { Seed }(\mathrm{s})\end{array}$ & $\begin{array}{l}\text { Number } \\
\text { Planted }\end{array}$ & $\%$ Survival \\
\hline 4 & 286 & Salvia dorrii & grayball sage & $\mathrm{p}$ & 5 & 100 \\
\hline 4 & 287 & Salvia dorrii & grayball sage & $\mathrm{p}$ & 5 & 100 \\
\hline 4 & 1592 & Salvia dorrii & grayball sage & $\mathrm{p}$ & 3 & 66.67 \\
\hline 5 & 266 & Salvia dorrii & grayball sage & $\mathrm{s}$ & 50 & 2 \\
\hline 5 & 267 & Salvia dorrii & grayball sage & $\mathrm{s}$ & 50 & 0 \\
\hline 5 & 268 & Salvia dorrii & grayball sage & s & 50 & 0 \\
\hline 5 & 1798 & Salvia dorrii & grayball sage & $\mathrm{p}$ & 5 & 20 \\
\hline 6 & 66 & Salvia dorrii & grayball sage & $\mathrm{p}$ & 3 & 100 \\
\hline 6 & 1590 & Salvia dorrii & grayball sage & $\mathrm{p}$ & 5 & 100 \\
\hline 6 & 1591 & Salvia dorrii & grayball sage & $\mathrm{p}$ & 5 & 100 \\
\hline 3 & 65 & Sedum leibergii & Leiberg's stonecrop & $\mathrm{p}$ & 1 & 0 \\
\hline 4 & 64 & Sedum leibergii & Leiberg's stonecrop & $\mathrm{p}$ & 1 & 0 \\
\hline 4 & 260 & Sedum leibergii & Leiberg's stonecrop & $\mathrm{s}$ & 50 & 0 \\
\hline 4 & 261 & Sedum leibergii & Leiberg's stonecrop & $\mathrm{s}$ & 50 & 0 \\
\hline 4 & 262 & Sedum leibergii & Leiberg's stonecrop & $\mathrm{s}$ & 50 & 0 \\
\hline 4 & 263 & Sedum leibergii & Leiberg's stonecrop & s & 50 & 0 \\
\hline 4 & 264 & Sedum leibergii & Leiberg's stonecrop & $\mathrm{s}$ & 50 & 0 \\
\hline 4 & 265 & Sedum leibergii & Leiberg's stonecrop & s & 50 & 0 \\
\hline 4 & 269 & Sedum leibergii & Leiberg's stonecrop & s & 50 & 0 \\
\hline 4 & 270 & Sedum leibergii & Leiberg's stonecrop & $\mathrm{s}$ & 50 & 0 \\
\hline 4 & 272 & Sedum leibergii & Leiberg's stonecrop & s & 50 & 0 \\
\hline 4 & 273 & Sedum leibergii & Leiberg's stonecrop & $\mathrm{s}$ & 50 & 0 \\
\hline 4 & 274 & Sedum leibergii & Leiberg's stonecrop & $\mathrm{s}$ & 50 & 0 \\
\hline 4 & 275 & Sedum leibergii & Leiberg's stonecrop & $\mathrm{s}$ & 50 & 0 \\
\hline 4 & 276 & Sedum leibergii & Leiberg's stonecrop & $\mathrm{s}$ & 50 & 0 \\
\hline 4 & 284 & Sedum leibergii & Leiberg's stonecrop & $\mathrm{p}$ & 4 & 0 \\
\hline 4 & 285 & Sedum leibergii & Leiberg's stonecrop & $\mathrm{p}$ & 1 & 0 \\
\hline 4 & 286 & Sedum leibergii & Leiberg's stonecrop & $\mathrm{p}$ & 1 & 0 \\
\hline
\end{tabular}


Table C.4. (contd)

\begin{tabular}{|c|c|c|c|c|c|c|}
\hline $\begin{array}{l}\text { Revegetation } \\
\text { Unit }\end{array}$ & Plot ID & Genus and Species & Common Name & $\begin{array}{l}\text { Plant (p) or } \\
\text { Seed (s) }\end{array}$ & $\begin{array}{l}\text { Number } \\
\text { Planted }\end{array}$ & $\%$ Survival \\
\hline 4 & 287 & Sedum leibergii & Leiberg's stonecrop & $\mathrm{p}$ & 1 & 0 \\
\hline 5 & 266 & Sedum leibergii & Leiberg's stonecrop & s & 50 & 0 \\
\hline 5 & 267 & Sedum leibergii & Leiberg's stonecrop & $\mathrm{s}$ & 50 & 0 \\
\hline 5 & 268 & Sedum leibergii & Leiberg's stonecrop & $\mathrm{s}$ & 50 & 0 \\
\hline 5 & 1798 & Sedum leibergii & Leiberg's stonecrop & $\mathrm{p}$ & 1 & 0 \\
\hline 6 & 66 & Sedum leibergii & Leiberg's stonecrop & $\mathrm{p}$ & 1 & 0 \\
\hline 6 & 277 & Sedum leibergii & Leiberg's stonecrop & $\mathrm{s}$ & 50 & 0 \\
\hline 6 & 278 & Sedum leibergii & Leiberg's stonecrop & $\mathrm{s}$ & 50 & 0 \\
\hline 6 & 279 & Sedum leibergii & Leiberg's stonecrop & $\mathrm{s}$ & 50 & 0 \\
\hline 6 & 1590 & Sedum leibergii & Leiberg's stonecrop & $\mathrm{p}$ & 1 & 0 \\
\hline 4 & 260 & Tetradymia canescens & gray horsebrush & $\mathrm{s}$ & 50 & 0 \\
\hline 4 & 261 & Tetradymia canescens & gray horsebrush & $\mathrm{s}$ & 50 & 0 \\
\hline 4 & 262 & Tetradymia canescens & gray horsebrush & $\mathrm{s}$ & 50 & 0 \\
\hline 4 & 263 & Tetradymia canescens & gray horsebrush & $\mathrm{s}$ & 50 & 0 \\
\hline 4 & 264 & Tetradymia canescens & gray horsebrush & $\mathrm{s}$ & 50 & 0 \\
\hline 4 & 265 & Tetradymia canescens & gray horsebrush & $\mathrm{s}$ & 50 & 0 \\
\hline 4 & 269 & Tetradymia canescens & gray horsebrush & s & 50 & 0 \\
\hline 4 & 270 & Tetradymia canescens & gray horsebrush & $\mathrm{s}$ & 50 & 0 \\
\hline 4 & 272 & Tetradymia canescens & gray horsebrush & s & 50 & 0 \\
\hline 4 & 273 & Tetradymia canescens & gray horsebrush & $\mathrm{s}$ & 50 & 0 \\
\hline 4 & 274 & Tetradymia canescens & gray horsebrush & $\mathrm{s}$ & 50 & 0 \\
\hline 4 & 275 & Tetradymia canescens & gray horsebrush & $\mathrm{s}$ & 50 & 0 \\
\hline 4 & 276 & Tetradymia canescens & gray horsebrush & S & 50 & 0 \\
\hline 5 & 266 & Tetradymia canescens & gray horsebrush & $\mathrm{s}$ & 50 & 0 \\
\hline 5 & 267 & Tetradymia canescens & gray horsebrush & $\mathrm{s}$ & 50 & 0 \\
\hline 5 & 268 & Tetradymia canescens & gray horsebrush & $\mathrm{s}$ & 50 & 0 \\
\hline 6 & 277 & Tetradymia canescens & gray horsebrush & s & 50 & 0 \\
\hline 6 & 278 & Tetradymia canescens & gray horsebrush & s & 50 & 0 \\
\hline
\end{tabular}


Table C.4. (contd)

\begin{tabular}{|c|c|c|c|c|c|c|}
\hline $\begin{array}{l}\text { Revegetation } \\
\text { Unit }\end{array}$ & Plot ID & Genus and Species & Common Name & $\begin{array}{l}\text { Plant (p) or } \\
\text { Seed (s) }\end{array}$ & $\begin{array}{l}\text { Number } \\
\text { Planted }\end{array}$ & $\%$ Survival \\
\hline 6 & 279 & Tetradymia canescens & gray horsebrush & $\mathrm{s}$ & 50 & 0 \\
\hline 2 & 282 & Achnatherum thurberianum & Thurber's needlegrass & $\mathrm{p}$ & 7 & 0 \\
\hline 2 & 1568 & Ericameria nauseosa & gray rabbitbrush & $\mathrm{s}$ & 1080 & 0.185 \\
\hline 2 & 1569 & Ericameria nauseosa & gray rabbitbrush & $\mathrm{s}$ & 1128 & 0 \\
\hline 2 & 1569 & Helianthus cusickii & Cusicks sunflower & $\mathrm{s}$ & 463 & 3.888 \\
\hline 2 & 1571 & Astragalus succumbens & crouching milkvetch & $\mathrm{s}$ & 475 & 3.789 \\
\hline 2 & 1571 & Ericameria nauseosa & gray rabbitbrush & $\mathrm{s}$ & 876 & 0.342 \\
\hline 2 & 1571 & Helianthus cusickii & Cusicks sunflower & $\mathrm{s}$ & 461 & 2.603 \\
\hline 2 & 1572 & Astragalus succumbens & crouching milkvetch & $\mathrm{s}$ & 475 & 1.895 \\
\hline 2 & 1572 & Ericameria nauseosa & gray rabbitbrush & $\mathrm{s}$ & 1186 & 0.675 \\
\hline 2 & 1572 & Helianthus cusickii & Cusicks sunflower & $\mathrm{s}$ & 484 & 1.033 \\
\hline 2 & 1573 & Astragalus succumbens & crouching milkvetch & $\mathrm{s}$ & 471 & 6.582 \\
\hline 2 & 1573 & Ericameria nauseosa & gray rabbitbrush & $\mathrm{s}$ & 1100 & 0.636 \\
\hline 2 & 1573 & Helianthus cusickii & Cusicks sunflower & $\mathrm{s}$ & 566 & 4.594 \\
\hline 2 & 1574 & Astragalus succumbens & crouching milkvetch & $\mathrm{s}$ & 471 & 1.486 \\
\hline 2 & 1574 & Ericameria nauseosa & gray rabbitbrush & $\mathrm{s}$ & 1421 & 0.141 \\
\hline 2 & 1574 & Helianthus cusickii & Cusicks sunflower & $\mathrm{s}$ & 518 & 1.351 \\
\hline 2 & 1575 & Astragalus succumbens & crouching milkvetch & $\mathrm{s}$ & 471 & 0 \\
\hline 2 & 1575 & Ericameria nauseosa & gray rabbitbrush & $\mathrm{s}$ & 1128 & 0.089 \\
\hline 2 & 1575 & Helianthus cusickii & Cusicks sunflower & $\mathrm{s}$ & 597 & 1.34 \\
\hline 2 & 1576 & Astragalus succumbens & crouching milkvetch & $\mathrm{s}$ & 500 & 1.6 \\
\hline 2 & 1576 & Ericameria nauseosa & gray rabbitbrush & $\mathrm{s}$ & 1102 & 0 \\
\hline 2 & 1576 & Helianthus cusickii & Cusicks sunflower & $\mathrm{s}$ & 500 & 2.6 \\
\hline 2 & 1577 & Astragalus succumbens & crouching milkvetch & $\mathrm{s}$ & 525 & 0.381 \\
\hline 2 & 1577 & Ericameria nauseosa & gray rabbitbrush & $\mathrm{s}$ & 1114 & 0 \\
\hline 2 & 1577 & Helianthus cusickii & Cusicks sunflower & $\mathrm{s}$ & 536 & 0.373 \\
\hline 2 & 1578 & Astragalus succumbens & crouching milkvetch & $\mathrm{s}$ & 459 & 1.961 \\
\hline 2 & 1578 & Ericameria nauseosa & gray rabbitbrush & $\mathrm{s}$ & 1107 & 0.181 \\
\hline
\end{tabular}


Table C.4. (contd)

\begin{tabular}{|c|c|c|c|c|c|c|}
\hline $\begin{array}{c}\text { Revegetation } \\
\text { Unit }\end{array}$ & Plot ID & Genus and Species & Common Name & $\begin{array}{l}\text { Plant }(\mathrm{p}) \text { or } \\
\text { Seed }(\mathrm{s})\end{array}$ & $\begin{array}{l}\text { Number } \\
\text { Planted }\end{array}$ & $\%$ Survival \\
\hline 2 & 1578 & Helianthus cusickii & Cusicks sunflower & $\mathrm{s}$ & 597 & 6.365 \\
\hline 2 & 1579 & Astragalus succumbens & crouching milkvetch & s & 484 & 1.446 \\
\hline 2 & 1579 & Ericameria nauseosa & gray rabbitbrush & $\mathrm{s}$ & 1316 & 0.152 \\
\hline 2 & 1579 & Helianthus cusickii & Cusicks sunflower & s & 546 & 2.564 \\
\hline 2 & 1580 & Astragalus succumbens & crouching milkvetch & $\mathrm{s}$ & 496 & 3.831 \\
\hline 2 & 1580 & Ericameria nauseosa & gray rabbitbrush & s & 1424 & 0.07 \\
\hline 2 & 1580 & Helianthus cusickii & Cusicks sunflower & $\mathrm{s}$ & 566 & 1.237 \\
\hline 2 & 1584 & Astragalus succumbens & crouching milkvetch & s & 463 & 0.432 \\
\hline 2 & 1584 & Ericameria nauseosa & gray rabbitbrush & s & 1061 & 0 \\
\hline 2 & 1585 & Astragalus succumbens & crouching milkvetch & s & 594 & 5.219 \\
\hline 2 & 1585 & Ericameria nauseosa & gray rabbitbrush & $\mathrm{s}$ & 977 & 0.512 \\
\hline 2 & 1585 & Helianthus cusickii & Cusicks sunflower & s & 482 & 0.207 \\
\hline 2 & 1586 & Astragalus succumbens & crouching milkvetch & s & 533 & 0.563 \\
\hline 2 & 1586 & Ericameria nauseosa & gray rabbitbrush & s & 881 & 0.114 \\
\hline 2 & 1587 & Astragalus caricinus & buckwheat milkvetch & s & 200 & 3 \\
\hline 2 & 1587 & Ericameria nauseosa & gray rabbitbrush & s & 652 & 0 \\
\hline 2 & 1587 & Helianthus cusickii & Cusicks sunflower & s & 390 & 0 \\
\hline 2 & 1588 & Astragalus succumbens & crouching milkvetch & s & 484 & 2.686 \\
\hline 2 & 1588 & Ericameria nauseosa & gray rabbitbrush & s & 1071 & 0.093 \\
\hline 2 & 1588 & Helianthus cusickii & Cusicks sunflower & $\mathrm{s}$ & 422 & 0.237 \\
\hline 2 & 1697 & Achnatherum thurberianum & Thurber's needlegrass & $\mathrm{p}$ & 7 & 57.14 \\
\hline 2 & 1697 & Achnatherum thurberianum & Thurber's needlegrass & $\mathrm{p}$ & 7 & 42.86 \\
\hline 2 & 1697 & Astragalus caricinus & buckwheat milkvetch & $\mathrm{p}$ & 25 & 44 \\
\hline 2 & 1697 & Astragalus caricinus & buckwheat milkvetch & $\mathrm{p}$ & 25 & 60 \\
\hline 2 & 1698 & Achnatherum thurberianum & Thurber's needlegrass & $\mathrm{p}$ & 7 & 28.57 \\
\hline 2 & 1698 & Astragalus caricinus & buckwheat milkvetch & $\mathrm{p}$ & 25 & 52 \\
\hline 3 & 65 & Nestotus stenophyllus & narrowleaf mock goldenweed & $\mathrm{p}$ & 1 & 0 \\
\hline 3 & 1589 & Ericameria nauseosa & gray rabbitbrush & s & 169 & 0 \\
\hline
\end{tabular}


Table C.4. (contd)

\begin{tabular}{|c|c|c|c|c|c|c|}
\hline $\begin{array}{l}\text { Revegetation } \\
\text { Unit }\end{array}$ & Plot ID & Genus and Species & Common Name & $\begin{array}{l}\text { Plant (p) or } \\
\text { Seed (s) }\end{array}$ & $\begin{array}{l}\text { Number } \\
\text { Planted }\end{array}$ & $\%$ Survival \\
\hline 4 & 260 & Astragalus conjunctus var. rickardii* & Dr. Bills locoweed & $\mathrm{s}$ & 50 & 0 \\
\hline 4 & 261 & Astragalus conjunctus var. rickardii* & Dr. Bills locoweed & $\mathrm{s}$ & 50 & 0 \\
\hline 4 & 261 & Nestotus stenophyllus & narrowleaf mock goldenweed & $\mathrm{s}$ & 50 & 0 \\
\hline 4 & 262 & Astragalus conjunctus var. rickardii* & Dr. Bills locoweed & $\mathrm{s}$ & 50 & 0 \\
\hline 4 & 263 & Astragalus conjunctus var. rickardii* & Dr. Bills locoweed & $\mathrm{s}$ & 50 & 0 \\
\hline 4 & 263 & Nestotus stenophyllus & narrowleaf mock goldenweed & $\mathrm{s}$ & 50 & 0 \\
\hline 4 & 264 & Astragalus conjunctus var. rickardii* & Dr. Bills locoweed & $\mathrm{s}$ & 50 & 0 \\
\hline 4 & 264 & Nestotus stenophyllus & narrowleaf mock goldenweed & $\mathrm{s}$ & 50 & 0 \\
\hline 4 & 265 & Astragalus conjunctus var. rickardii* & Dr. Bills locoweed & $\mathrm{s}$ & 50 & 0 \\
\hline 4 & 265 & Nestotus stenophyllus & narrowleaf mock goldenweed & $\mathrm{s}$ & 50 & 0 \\
\hline 4 & 274 & Nestotus stenophyllus & narrowleaf mock goldenweed & s & 50 & 0 \\
\hline 4 & 275 & Nestotus stenophyllus & narrowleaf mock goldenweed & $\mathrm{s}$ & 50 & 0 \\
\hline 4 & 276 & Nestotus stenophyllus & narrowleaf mock goldenweed & $\mathrm{s}$ & 50 & 0 \\
\hline 4 & 284 & Nestotus stenophyllus & narrowleaf mock goldenweed & $\mathrm{p}$ & 1 & 0 \\
\hline 4 & 285 & Nestotus stenophyllus & narrowleaf mock goldenweed & $\mathrm{p}$ & 1 & 0 \\
\hline 4 & 286 & Nestotus stenophyllus & narrowleaf mock goldenweed & $\mathrm{p}$ & 1 & 0 \\
\hline 4 & 287 & Nestotus stenophyllus & narrowleaf mock goldenweed & $\mathrm{p}$ & 1 & 0 \\
\hline 5 & 266 & Astragalus conjunctus var. rickardii* & Dr. Bills locoweed & $\mathrm{s}$ & 50 & 0 \\
\hline 5 & 266 & Nestotus stenophyllus & narrowleaf mock goldenweed & $\mathrm{s}$ & 50 & 0 \\
\hline 5 & 267 & Astragalus conjunctus var. rickardii* & Dr. Bills locoweed & $\mathrm{s}$ & 50 & 0 \\
\hline 5 & 267 & Nestotus stenophyllus & narrowleaf mock goldenweed & $\mathrm{s}$ & 50 & 0 \\
\hline
\end{tabular}


Table C.4. (contd)

\begin{tabular}{|c|c|c|c|c|c|c|}
\hline $\begin{array}{l}\text { Revegetation } \\
\text { Unit }\end{array}$ & Plot ID & Genus and Species & Common Name & $\begin{array}{l}\text { Plant (p) or } \\
\text { Seed (s) }\end{array}$ & $\begin{array}{l}\text { Number } \\
\text { Planted }\end{array}$ & $\%$ Survival \\
\hline 5 & 268 & Nestotus stenophyllus & narrowleaf mock goldenweed & $\mathrm{s}$ & 50 & 0 \\
\hline 5 & 1798 & Nestotus stenophyllus & narrowleaf mock goldenweed & $\mathrm{p}$ & 1 & 0 \\
\hline 6 & 66 & Nestotus stenophyllus & narrowleaf mock goldenweed & $\mathrm{p}$ & 1 & 0 \\
\hline 6 & 277 & Nestotus stenophyllus & narrowleaf mock goldenweed & $\mathrm{s}$ & 50 & 0 \\
\hline 6 & 278 & Nestotus stenophyllus & narrowleaf mock goldenweed & $\mathrm{s}$ & 50 & 0 \\
\hline 6 & 279 & Nestotus stenophyllus & narrowleaf mock goldenweed & $\mathrm{s}$ & 50 & 0 \\
\hline 6 & 1590 & Nestotus stenophyllus & narrowleaf mock goldenweed & $\mathrm{p}$ & 1 & 0 \\
\hline 6 & 1591 & Nestotus stenophyllus & narrowleaf mock goldenweed & $\mathrm{p}$ & 1 & 0 \\
\hline
\end{tabular}




\section{Appendix D}

\section{Restoration After Construction Disturbance on the Arid Lands Ecology Reserve}




\title{
Appendix D
}

\section{Restoration After Construction Disturbance on the Arid Lands Ecology Reserve}

\author{
Steven Link and Rico Cruz \\ Department of Science and Engineering \\ Confederated Tribes of the Umatilla Indian Reservation \\ 46411 Timine Way \\ Pendleton, OR 97801 \\ stevenlink@ctuir.org \\ stevenlink123@yahoo.com \\ 509-948-0054
}

\section{D.1 Introduction}

The Confederated Tribes of the Umatilla Indian Reservation (CTUIR) assisted the Pacific Northwest National Laboratory (PNNL) with revegetation of areas of the Fitzner/Eberhardt Arid Lands Ecology (ALE) Reserve affected by decommissioning of buildings and infrastructure and debris cleanup actions. The CTUIR provided technical services to assist in planting, seeding, watering, and monitoring revegetation actions performed during FY 2011 on the Arid Lands Ecology Reserve. We accomplished the following:

1. Propagated approximately 2600 selected native forb and shrub plants from locally collected seeds in a greenhouse setting for transplant as described in the ALE revegetation plan.

2. Transplanted plant material grown by the CTUIR into the revegetation units in February through early April 2011.

3. Conducted one watering of transplanted materials at Rattlesnake Springs.

4. Transplanted 134 additional shrub (sagebrush) seedlings into the Gas well road.

5. Performed weed and weed seed removal along a linear strip at the top of Rattlesnake Ridge.

6. Performed hand broadcasting of selected native forb/shrub seeds in coordination with the PNNL ecologists and WildLands, Inc.

7. Assisted in the development of a monitoring strategy and methods for the revegetation units.

8. Assisted in monitoring success of the revegetation efforts. 


\section{D.2 Methods}

\section{D.2.1 Study Area}

The study area is on the ALE Reserve of the Hanford National Monument in south-central Washington. The average annual precipitation is $16 \mathrm{~cm}$, occurring mostly in the autumn and winter (Stone et al. 1983). The soil is classified as a Warden Silt Loam more than $1 \mathrm{~m}$ deep (Hajek 1966).

\section{D.2.2 Seed Collection and Processing}

Seeds were collected by hand in and near the restoration areas from 2005 through summer 2010. Seeds were placed in paper bags and stored at room temperature, dry, and in the dark until cleaned. Seeds that were being predated by insects or that may be predated were frozen before cleaning. Seed cleaning was done by hand separating debris from the seed. This was done using screens, tools to rub the flower heads and seed against the screens, fans, and tweezers.

\section{D.2.3 Plant Propagation}

Plants were grown in a climate-controlled glasshouse. Lighting was supplemented with $1000 \mathrm{~W}$ metal halide grow lights. Seeds were sown in $164 \mathrm{ml}$ Ray Leach Cone-tainer cells that were filled with a mixture of $46.1 \%$ potting soil with slow release fertilizer (Miracle Grow potting mix), $46.1 \%$ sand, 3.8\% perlite, and 3.8\% vermiculite. Seeds were sown between November 2, 2010 and January 31, 2011. Tubes filled with wetted soil were planted with seeds just below the surface. The number of seeds planted in each pot ranged from 1 to 10 depending on the species and the amount of available seed. Each 98-cell rack was an experimental unit. Irrigation was automated with frequency and amount changes during the growth period to optimize germination and growth. Irrigation was more frequent during seed germination and less frequent during growth. Plants were further fertilized using Scott Miracle-Gro Nursery Select (20N-20P-20K). Fertilizer was dissolved as $10 \mathrm{ml}$ solid in 7.61 water and applied by pouring over about 800 cells. Fertilizer was applied four times from December 22, 2010 to January 27, 2011. Date of planting, observation date, and the number of seedlings were recorded. Twenty-six hundred seedlings were grown for planting in the late winter and early spring of 2011.

\section{D.2.4 Plant Installation and Seeding}

Plants were installed in 21 plots and areas across the 7 site units. Installation occurred from March 7 to April 7, 2011, proceeding from low to high elevation in time. Installation at Rattlesnake Springs (Unit 1) was done throughout the area with 300 spiny hopsage (Grayia spinosa). Installation of snowy mountain dandelion (Agoseris grandiflora) and buckwheat milkvetch (Astragalus caricinus) occurred in two small patches for each species. The patches are approximately $4 \mathrm{~m}^{2}$. Installation at the Gas well road (Unit 2) was done for approximately $120 \mathrm{~m}$ along the road with 134 Big sagebrush (Artemisia tridentata). Plots in Unit 2 were circular with a radius of $5 \mathrm{~m}$. Each plot was marked at the center with a spike or rebar with a numbered aluminum tag that was wired into the ground. Plots in Units 3 through 6 were circular with a radius of $4 \mathrm{~m}$ except where circumstances required planting in other irregular patterns. All plots were marked at the center with a spike or rebar with a numbered aluminum tag that was wired into the ground. Plants in Unit 7 were planted in three locations. Western horsemint (Agastache occidentalis) 
and woolly-pod milkvetch (Astragalus purshii) were installed in one marked irregular plot. Western white clematis (Clematis ligusticifolia) were planted in two locations and marked with flagging. Planting was done with dibbles and hand tools in the softer soils at low elevation. Planting was done with pry bars and dibbles in the lithosols at high elevation. The dibble is $91 \mathrm{~cm}$ long, the planting blade is $30 \mathrm{~cm}$ long, and the width of the planting is $7.6 \mathrm{~cm}$. Plants were well watered just before installation. Planting was done by separating the seedling from the pot, immediately placing the seedling in the hole, and packing soil into the hole. Separation of the soil and plant from the pot was done by tapping on the top of the plastic tube. Care was taken to place the entire root system linearly in the hole without turning root tips up. Soils were packed around the seedlings to eliminate air pockets near roots. The upper soil surface of seedlings in tubes was placed below the surface of site soil upon planting and covered with site soil. In the lithosols at high elevation, rocks were used to create a protected space for the seedlings. Rocks were circled around the plant especially on the upwind side of the seedling. Soils were wet throughout the planting profile. Plants were not given supplemental water except at Rattlesnake Springs where watering was done on April 18. Each plant was watered by opening a hole next to a seedling with a spike and pouring approximately $250 \mathrm{ml}$ water into the hole. The number of each species installed in each plot is noted in Appendix C. Pictures were taken of each plot.

Seeding was done in 42 plots across 6 of the 7 units. Seeding occurred from January 19 through February 11, 2011, proceeding from low to high elevation in time. Plots were circles with varying radii. Plots at low elevation had radii ranging from 2.6 to $1.96 \mathrm{~m}$ depending on the number of seed for each plot. Plots at higher elevation had radii ranging from 1.39 to $0.544 \mathrm{~m}$ depending on the number of seed available (Appendix C). Seed plots were marked in the center with a numbered metal tag wired into the ground. Some plots also have a spike or rebar at the center. Seeding was done by roughing the surface with shoes, hand broadcasting the seed, covering the seed with soil, walking on the surface to push the seed in, and then applying mulch when available. Pictures were taken of each plot.

\section{D.2.5 Cheatgrass Control at High Elevation}

As a result of re-contouring a road along the northeast face of Rattlesnake Mountain, there has been an invasion of significant numbers of cheatgrass (B. tectorum). The population was documented and seed removal attempted by mechanical means and by hand. Documentation of plant communities on the disturbance was done on June 14, 2011, by determining cover of each species along with other surface characters. Cover was determined using a tape measure (Bonham 1989; Elmore et al. 2003; Link et al. 2006) and identifying the first observed (tallest) cover type at each $0.1-\mathrm{m}$ hash mark on the tape. The tape was stretched tightly between two pieces of rebar metal stakes in the ground at the ends of any transect. Six locations along the road were documented. Each transect was marked with numbered metal tags wired into the ground. Each transect crosses the disturbance at a right angle to the long dimension of the disturbance (Figure D.1). There are 77 observations in each transect.

Control of the population was initiated on June 15 and finished on August 2, 2011. Plants were cut with a gasoline-powered Honda weed eater or pulled by hand. Plants and seed were picked up using a Stihl vacuum along with rakes and hand picking. Trash bags were filled with the material and removed from the area. The bags were counted. Up to four people conducted control for several days. 


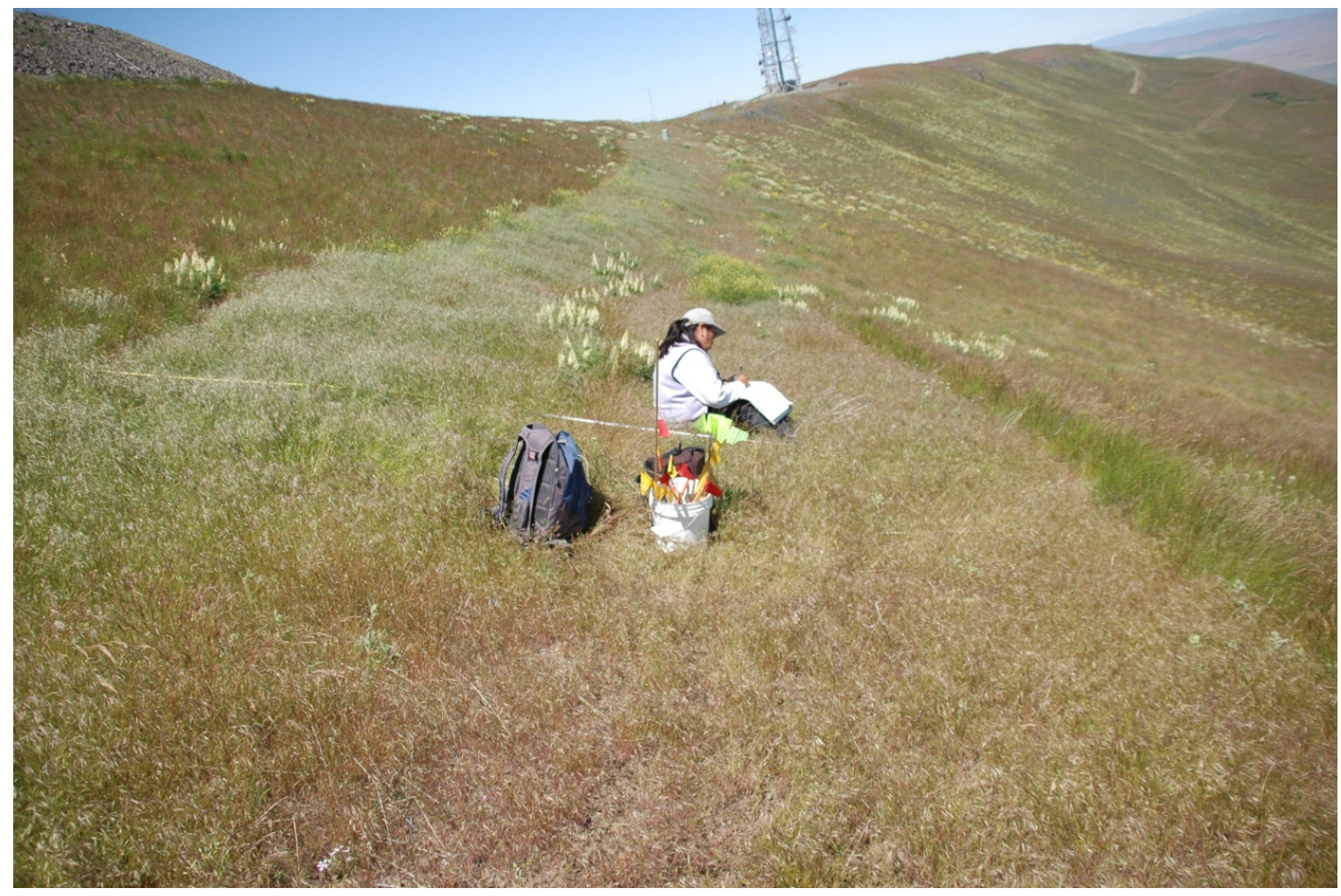

Figure D.1. Cover Observations in the Cheatgrass Invasion Area on June 14, 2011

\section{D.2.6 Monitoring}

Field monitoring occurred between June 17 and July 14, 2011. In the planted plots, all individuals of all species were counted and condition noted (green and growing, stressed, dead, flowering status). In the seeded plots, all seedlings of applied species were counted and condition noted. Enumeration was done by sectioning a plot into small parcels to facilitate accurate counts. Percent cover of each species and that of litter, soil, soil cryptogams, and rocks was estimated for each plot. Two observers were used for every plot. Pictures were taken of each plot after assessment.

\section{D.2.7 Data analysis}

Percent germination in the greenhouse was computed by dividing the number of live seedlings by the number of seeds sown and multiplying by 100 . Survivorship in the field was determined by counting the number of live seedlings within each plot and computing percent survival based the number of individuals planted in each plot. Percent establishment for seeded species was determined by dividing the observed number of individuals of each species by the number of seed applied. Percent of plants in each condition class was also computed. Data were analyzed using JMP software (SAS Institute 2002) to compute means and one standard error of the mean. Survival data are presented in Appendix C (Table C.4). 


\section{D.3 Results}

Table D.1. Mean Maximal Germination ( \pm 1 standard error of the mean [sem]) and sample size (n) of Perennial Native Species Used for Transplanting

\begin{tabular}{|c|c|c|c|c|c|}
\hline $\begin{array}{l}\text { Family } \\
\text { Species }\end{array}$ & Common Name & Life Form & $\begin{array}{c}\text { Mean } \\
\text { Maximum } \\
\text { Germination } \\
(\%)\end{array}$ & $1 \mathrm{sem}$ & $\mathrm{n}$ \\
\hline \multicolumn{6}{|l|}{ Asteraceae } \\
\hline Agoseris grandiflora & Large-flowered agoseris & herb & 25.5 & 7.92 & 6 \\
\hline Artemisia tridentata & Big sagebrush & shrub & 6.02 & 2.97 & 5 \\
\hline Balsamorhiza rosea & Rosy balsamroot & herb & 6.12 & 1.02 & 3 \\
\hline Crepis modocensis & Low hawksbeard & herb & 31.3 & & 1 \\
\hline Ericameria nauseosa & Gray rabbitbrush & shrub & 1.02 & & 1 \\
\hline Erigeron linearis & Desert yellow daisy & herb & 20.8 & 1.09 & 3 \\
\hline Erigeron piperianus & Piper's daisy & herb & 55.9 & 9.68 & 10 \\
\hline Erigeron poliospermus & Cushion fleabane & herb & 4.42 & 1.97 & 2 \\
\hline $\begin{array}{l}\text { Eriophyllum lanatum var. } \\
\text { Integrifolum }\end{array}$ & Woolly sunflower & herb & 0 & & 1 \\
\hline Nestotus stenophyllus & narrowleaf mock goldenweed & herb & 78.4 & & 1 \\
\hline Tetradymia canescens & gray horsebrush & shrub & 0 & & 1 \\
\hline \multicolumn{6}{|l|}{ Chenopodiaceae } \\
\hline Grayia spinosa & Hopsage & shrub & 35.0 & 1.96 & 3 \\
\hline \multicolumn{6}{|l|}{ Crassulaceae } \\
\hline Sedum leibergii & Leiberg's sedum & & 17.9 & & 1 \\
\hline \multicolumn{6}{|l|}{ Cruciferae } \\
\hline Phoenicaulis cheiranthoides & Daggerpod & herb & 0 & & 1 \\
\hline \multicolumn{6}{|l|}{ Grossulariaceae } \\
\hline Ribes cereum & squaw currant & shrub & 0 & & 1 \\
\hline \multicolumn{6}{|l|}{ Laminaceae } \\
\hline Agastache occidentalis & western horsemint & herb & 58.2 & & 1 \\
\hline Salvia dorrii & Purple sage & shrub & 12.6 & 4.42 & 3 \\
\hline \multicolumn{6}{|l|}{ Leguminosae } \\
\hline Astragalus caricinus & Buckwheat milkvetch & herb & 63.9 & 3.27 & 5 \\
\hline Astragalus conjunctus var. rick & Dr. Bill's locoweed & herb & 0 & & 1 \\
\hline Astragalus purshii & Woolly-pod milkvetch & herb & 61.9 & 8.66 & 5 \\
\hline Lupinus sericeus & Silky lupine & herb & 31.47 & 4.28 & 4 \\
\hline \multicolumn{6}{|l|}{ Poaceae } \\
\hline Achnatherum thurberianum & Thurber's needlegrass & bunchgrass & 61.9 & & 1 \\
\hline Leymus cinereus & Giant wildrye & bunchgrass & 0 & & 1 \\
\hline \multicolumn{6}{|l|}{ Polemoniaceae } \\
\hline Phlox hoodii & Hood's phlox & subshrub & 5.56 & & 1 \\
\hline Phlox longifolia & Longleaf phlox & subshrub & 55.9 & & 1 \\
\hline
\end{tabular}


Table D.1. (contd)

\begin{tabular}{|c|c|c|c|c|c|}
\hline $\begin{array}{l}\text { Family } \\
\text { Species }\end{array}$ & Common Name & Life Form & $\begin{array}{c}\text { Mean } \\
\text { Maximum } \\
\text { Germination } \\
(\%)\end{array}$ & $1 \mathrm{sem}$ & $\mathrm{n}$ \\
\hline \multicolumn{6}{|l|}{ Polygonaceae } \\
\hline Eriogonum sphaerocephalum & rock buckwheat & shrub & 10.7 & 6.50 & 3 \\
\hline Eriogonum thymoides & Thyme buckwheat & shrub & 9.95 & 1.69 & 4 \\
\hline \multicolumn{6}{|l|}{ Portulacaceae } \\
\hline Phemeranthus spinescens & spiny fameflower & herb & 78.4 & & 1 \\
\hline \multicolumn{6}{|l|}{ Ranunculaceae } \\
\hline Clematis ligusticifolia & western white clematis & vine & 13.3 & & 1 \\
\hline \multicolumn{6}{|l|}{ Scrophulariaceae } \\
\hline Penstemon speciosus & showy beardtongue & herb & 5.37 & & 1 \\
\hline \multicolumn{6}{|l|}{ Crassulaceae } \\
\hline Sedum leibergii & Leiberg's stonecrop & herb & 17.9 & & 1 \\
\hline
\end{tabular}

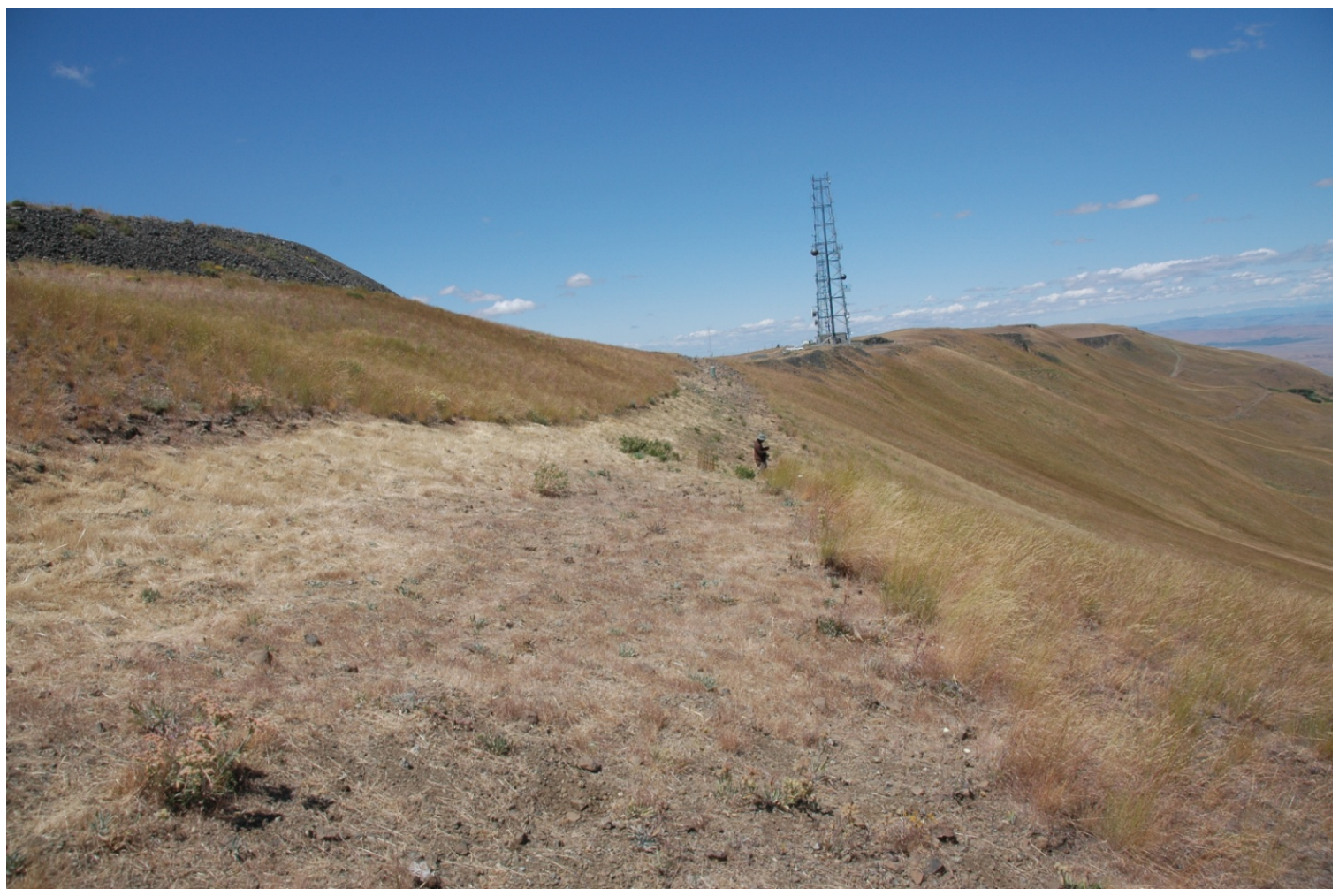

Figure D.2. Partially Controlled Cheatgrass Population on July 13, 2011 


\section{D.4 References}

Bonham CD. 1989. Measurements for Terrestrial Vegetation. John Wiley \& Sons, New York.

Elmore AJ, JF Mustard, and SJ Manning. 2003. "Regional patterns of plant community response to changes in water: Owens Valley, California." Ecological Applications 13(2):443-460.

Hajek BF. 1966. Soil Survey, Hanford Project in Benton County, Washington. BNWL-243, Pacific Northwest Laboratory, Richland, Washington.

Link SO, CW Keeler, RW Hill, and E Hagen. 2006. "Bromus tectorum cover mapping and fire risk." International Journal of Wildland Fire 15(1):113-119.

SAS Institute. 2002. JMP Statistics and Graphics Guide, Version 5. Cary, SAS Institute Inc.

Stone WA, JM Thorp, OP Gifford, and DJ Hoitink. 1983. Climatological Summary for the Hanford Area. PNL-4622, Pacific Northwest Laboratory, Richland, Washington. 


\section{Distribution}

No. of

Copies

\section{OFFSITE}

R Cruz (PDF)

Department of Science and Engineering

Confederated Tribes of the Umatilla Indian

Reservation

P.O. Box 638

Pendleton, OR 97801

S Link (PDF)

Department of Science and Engineering

Confederated Tribes of the Umatilla Indian

Reservation

P.O. Box 638

Pendleton, OR 97801

ONSITE

DOE Richland Operations Office

A Farabee

(PDF)

HB Hathaway

(PDF)

(PDF)

(PDF)

(PDF)

(PDF)
AL Rodriguez

DC Ward

MK Wright
No. of

\section{Copies}

\section{CH2M HILL Plateau Remediation}

Company

GM Banks

(PDF)

DL Norman

(PDF)

L Oates

(PDF)

DR Turlington

(PDF)

Mission Support Alliance

$\begin{array}{ll}\text { AL Johnson } & \text { (PDF) } \\ \text { MR Sachschewsky } & \text { (PDF) } \\ \text { Pacific Northwest National Laboratory } \\ \text { RL Dirkes } & \text { (PDF) } \\ \text { JL Downs } & \text { (PDF) } \\ \text { RE Durham } & \text { (PDF) } \\ \text { SA McKee } & \text { (PDF) }\end{array}$

Distr.1 


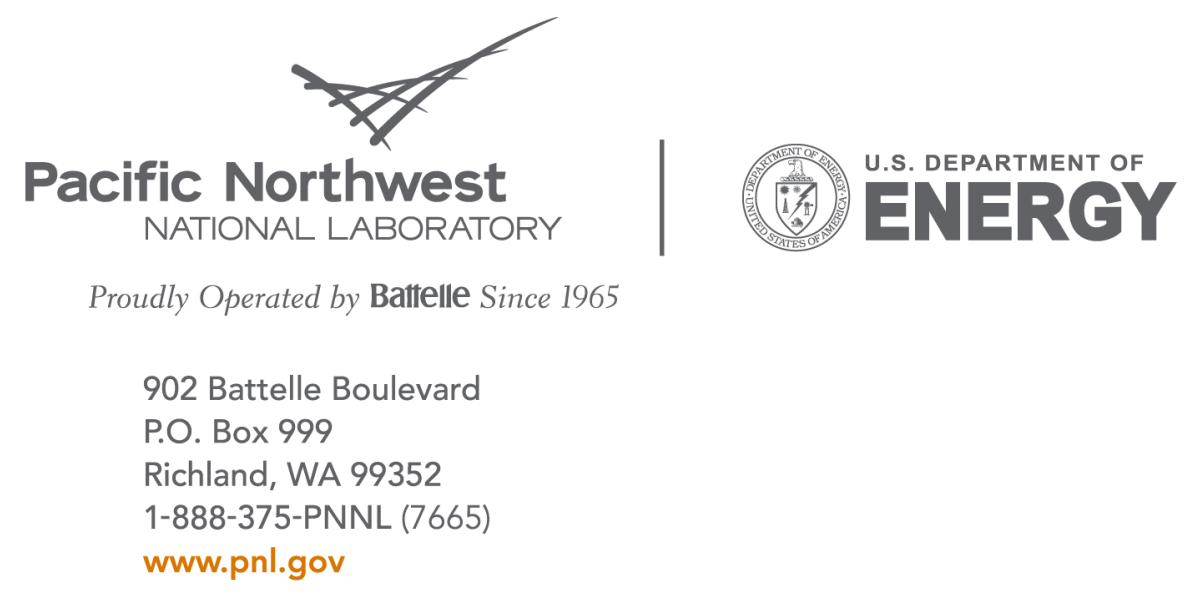

